\title{
Assessing the observed impact of anthropogenic climate change
}

Gerrit Hansen 


\section{Assessing the observed impact of anthropogenic climate change}

Gerrit Hansen 


\section{Thesis committee}

\section{Promotor}

Prof. Dr Rik Leemans

Professor of Environmental Systems Analysis

Wageningen University

\section{Co-promotor}

Prof. Dr Maximilian Auffhammer

George Pardee Jr. Chair in Sustainable Development

University of California, Berkeley, USA

\section{Other members}

Dr Dim Coumou, Potsdam Institute for Climate Impact Research, Germany

Prof. Dr Martin Herold, Wageningen University

Prof. Dr Jan Minx, Mercator Institute for Climate Change and Global Commons, Berlin, Germany

Prof. Dr Hans Otto Pörtner, Alfred-Wegener-Institute for Polar and Marine Research, Bremerhaven, Germany

This research was conducted under the auspices of the Graduate School for Socio-Economic and Natural Sciences of the Environment (SENSE) 


\section{Assessing the observed impact of anthropogenic climate change}

\section{Gerrit Hansen}

Thesis

submitted in fulfilment of the requirements for the degree of doctor

at Wageningen University

by the authority of the Rector Magnificus

Prof. Dr A.P.J. Mol,

in the presence of the

Thesis Committee appointed by the Academic Board

to be defended in public

on Monday 14 September 2015

at 11 a.m. in the Aula. 


\section{Gerrit Hansen}

Assessing the observed impact of anthropogenic climate change 186 pages.

PhD thesis, Wageningen University, Wageningen, NL (2015)

with references, with summary in English

ISBN 978-94-6257-429-8 
Table of Contents

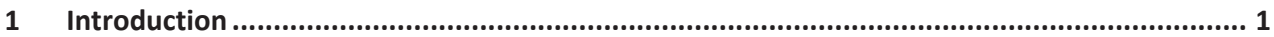

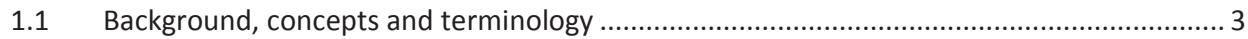

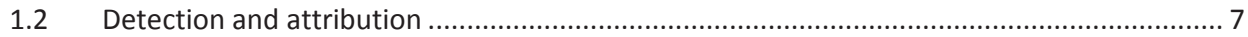

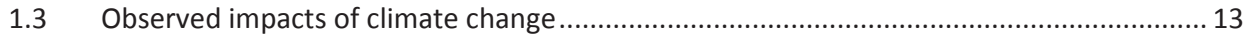

1.4 Attribution of observed climate change to human influence........................................... 15

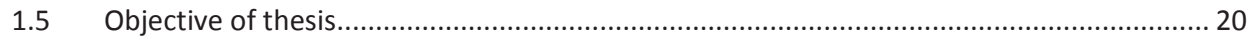

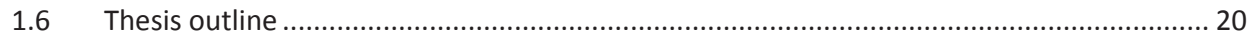

2 Linking local impacts to changes in climate - a guide to attribution ......................................23

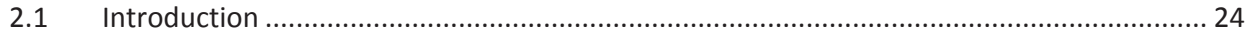

2.2 The five steps of an impact detection and attribution analysis ..........................................26

2.3 Impact attribution assessments - examples from human and managed systems...............32

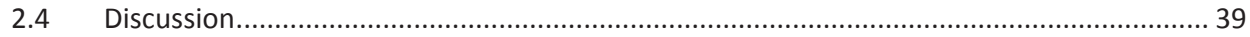

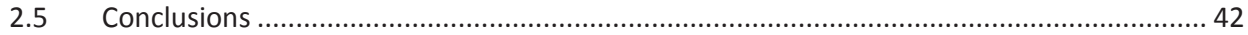

3 On the attribution of a single event to climate change ........................................................43

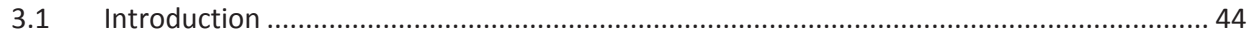

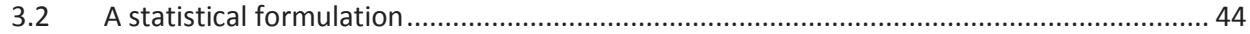

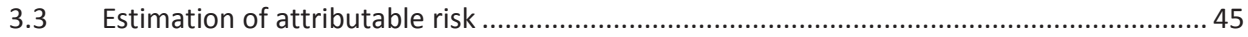

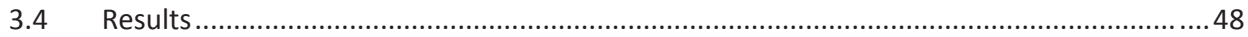

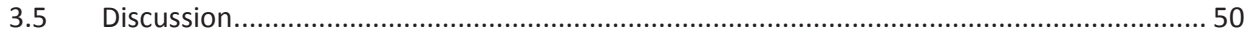

$4 \quad$ The evolution of the evidence base for observed impacts of climate change .........................51

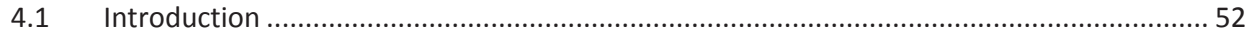

4.2 The evolution of the evidence base for observed impacts of climate change ......................53

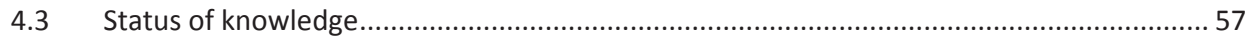

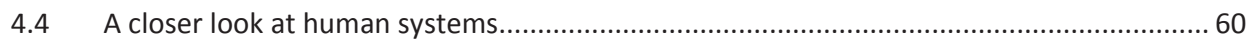

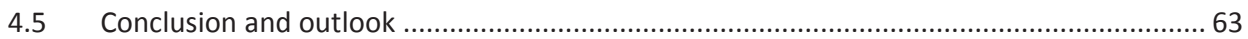


5 Attributing observed climate change impacts to human influence .......................................65

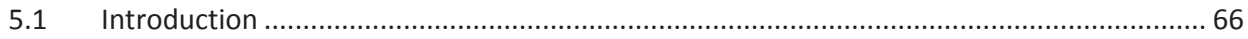

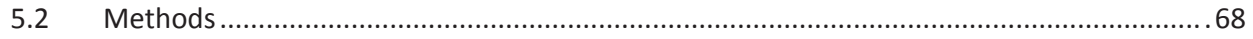

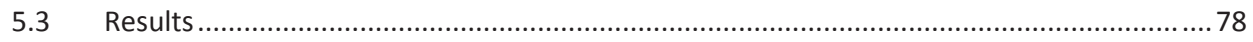

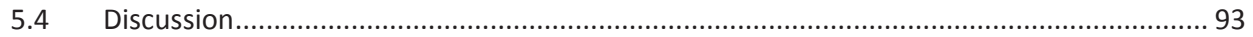

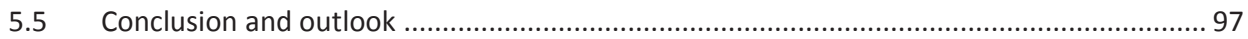

6 Global distribution of observed climate change impacts .....................................................99

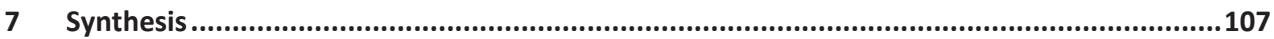

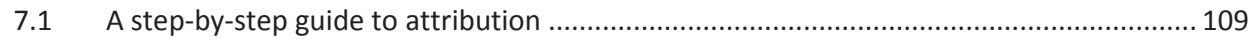

7.2 Impact attribution in the context of climate variability and extreme weather events ......113

7.3 Observed effects of climate change on natural and human systems..............................116

7.4 The relationship between observed impacts and anthropogenic forcing ......................... 120

7.5 Detection and attribution in the context of science-policy assessments ...........................126

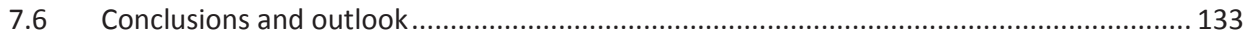

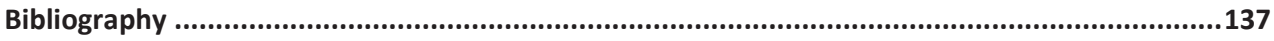

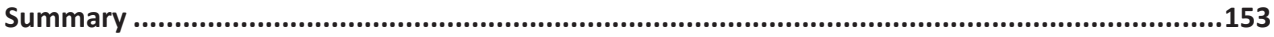

Annex A - Systematic attribution method for Chapter 5 .....................................................................157

Annex B - Tabulated inputs and results from Chapter 5 ....................................................................176

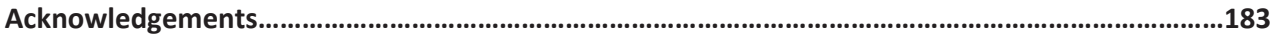

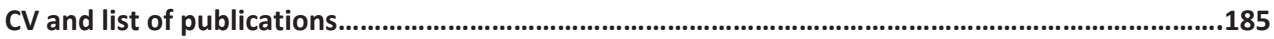




\section{Symbols and acronyms}

AR4

AR5

CMIP5

$\mathrm{CO}_{2}$

ENSO

$\mathrm{FAQ}$

FAR

GCM

GCOS

IPCC

$p$

$\hat{p}$

$\mathrm{pH}$

SPM

SAR

SREX

TAR

TS

UN

UNFCCC

US

WBGT

WGI

WGII

WGIII
Fourth Assessment Report (of the IPCC)

Fifth Assessment Report (of the IPCC)

Coupled Model Intercomparison Project 5

Carbon dioxide

El Niño Southern Oscillation

Frequently asked questions

First Assessment Report (of the IPCC)

General Circulation Model

Global Climate Observing System

Intergovernmental Panel on Climate Change

Probability

Maximum likelihood estimate of probability $p$

Dimensionless measure for acidity of a liquid $\left(\mathrm{pH}=-\log _{10}\left(\mathrm{H}^{+}\right)\right.$, the negative logarithm of hydrogen ion concentration)

Summary for Policymakers

Second Assessment Report (of the IPCC)

IPCC Special report on managing the risk of extreme events and disasters to advance climate change adaptation

Third Assessment Report (of the IPCC)

Technical Summary

United Nations

United Nations Framework Convention on Climate Change

United States (of America)

Wet Bulb Globe Temperature

IPCC working group I (The physical science basis)

IPCC working group II (Impacts, vulnerability and adaptation)

IPCC working group III (Mitigation) 


\section{Introduction}

Global climate change is unequivocal (IPCC 2013a). The question whether and to what extent the impacts of anthropogenic climate change can already be observed in natural and human systems is of high public interest. While recent climate change impacts on natural water resources and ecosystems worldwide have evidently been detected, gaps persist for many human and managed systems, and regional coverage remains unbalanced (Cramer et al 2014). The direct attribution of such observed impacts to anthropogenic forcing is very difficult and consequently, studies documenting the effect of anthropogenic forcing for observed impacts are very rare. However, a link between observed impacts and anthropogenic climate change has been shown globally (Rosenzweig et al 2008; Rosenzweig and Neofotis 2013).

Detection and attribution exercises address the question whether something has changed and examine the causes of that change. The term detection refers to the process of identifying a change in a system with statistical confidence. Attribution establishes causal relations between a detected change and one or more drivers (Hegerl et al 2010; Stone et al 2013). In the context of climate change research, existing concepts of detection and attribution mostly apply to the disciplinary analysis of well-bounded systems, i.e. the global climate system, and therefore need to be adapted to be suitable in the interdisciplinary and multifactorial context of most impact systems (Stone et al 2009; Stone et al 2013).

Impacts manifesting on a local or regional scale within complex, dynamic systems, such as managed natural and human systems, are difficult to detect. The behaviour of such systems in the absence of climate change is usually non-stationary and the effects of a sustained trend in a climate variable may be masked, compounded, overcompensated or amplified by the effects of other drivers, including unconscious or explicit adaptation to observed or expected climate change. A core part of any detection and attribution exercise consists in the explicit formulation of causal chains and in testing these against the influence of other drivers and confounding factors, i.e. factors that are not included in the analysis but may distort the result, such as measurement error, or unaccounted drivers of change. The establishment of causal relations is very challenging in multifactorial environments and more so within the field of qualitative social research, where quantitative data may be absent or limited to a very narrow set of case studies. Some social science scholars oppose attempts of to 'isolate' climatic factors as drivers from the intersecting web of drivers that constitute the dynamic of societies and social systems (Hulme 2010; Diemberger et al 2012). Therefore, to allow for a concept of detection and attribution to be applicable across a wide range of disciplines, 
the approach originally developed in the context of physical climate change needs to be amended but without compromising scientific rigour (Stone et al 2013; Hansen et al 2013).

The end point of an attribution exercise in the context of impact will often be a change in a climate variable as opposed to other drivers of change, while detection and attribution in the context of climate science focusses on distinguishing the role of anthropogenic forcing from that of other external acts of forcing and natural variability (Hegerl et al 2011; Bindoff et al 2013).

In the context of extreme events, such as severe storms or heat waves and their impacts, event attribution examining the role of anthropogenic forcing in the occurrence and intensity of specific events is increasingly being carried out by scientists (Rahmstorf and Coumou 2011; Peterson et al 2012; Peterson et al 2013b; Lott et al 2013). However, understanding both the statistical foundation of single event attribution (Stott et al 2013) and the interplay of climate hazard, exposure and vulnerability for the severity of the actual impact event (Allen et al 2007; Huggel et al 2013) is crucial in order to explain the role of a changing climate in loss and damage experienced during periods of extreme weather.

Despite substantial new evidence for some sectors, most notably food production, a gap exists in the literature concerning observed impacts of climate change on human systems (Rosenzweig and Neofotis 2013; Cramer et al 2014). Besides the above-mentioned issues and factual constraints related to data availability and model performance (Hegerl et al 2010; Rosenzweig and Neofotis 2013) other possible reasons for the weak representation of some impact systems emerged during the first phase of this project ${ }^{1}$. For example, parts of the relevant literature lack clarity in distinguishing observed impacts from vulnerability or sensitivity, and in separating climate variability from climate change. Also, much literature identifies sensitivities to climate and infers potential outcomes of future climate scenarios, yet this is hardly ever done for the past. Complementary exercises similar to projections of future impacts could be undertaken using a hypothetical past stable climate, followed by comparison with observations to draw conclusions about impacts that have already manifested. This suggests that the gap in research could be alleviated to some extent if a clear framework for the attribution of impacts were to be followed and if research priorities were shifted to include the understanding of past effects.

This chapter introduces terms and concepts that are used throughout this thesis and presents the research questions and thesis outline. In Section 1.1, some background to this thesis is provided and

\footnotetext{
${ }^{1}$ The first phase of this thesis was carried out during my position as a chapter scientist supporting the chapter "detection and attribution of observed impacts" (Cramer et al 2014) of the working group II contribution to the Intergovernmental Panel on Climate Change's (IPCC) Fifth Assessment Report (IPCC 2014a). This entailed extensive literature review, expert solicitation and exchange with researchers from different fields.
} 
basic concepts are defined. Section 1.2 reviews the development of approaches to 'detection and attribution' and highlights the differences between climate attribution and impact attribution. Section 1.3 briefly summarizes the status of knowledge regarding observed impacts of climate change and Section 1.4 provides an overview about observed trends in climate averages (1.4.1) and climate variability (1.4.2) and their respective attribution to anthropogenic forcing. In Section 1.5, the objective of this thesis and the research questions are presented. Finally, the structure of the thesis is introduced in Section 1.6.

\subsection{Background, concepts and terminology}

This thesis uses IPCC definitions and uncertainty measures and relies on IPCC assessments as input for several chapters. Therefore, the following sections briefly introduce the IPCC and its role in climate change research, and the IPCC confidence language. Concepts central to this thesis are also defined before turning to 'detection and attribution' in Section 1.2.

\subsubsection{The Intergovernmental Panel on Climate Change}

The Intergovernmental Panel on Climate Change is the leading international body for the assessment of climate change. It was established by the United Nations Environment Programme and the World Meteorological Organization in 1988 to provide a clear scientific view on the current state of knowledge in climate change and its potential environmental and socio-economic impacts. The IPCC is a scientific body under the auspices of the United Nations (UN). It reviews and assesses the most recent scientific, technical and socio-economic information produced worldwide relevant to the understanding of climate change. The IPCC does not conduct any research nor does it monitor climate-related data or parameters (http://www.ipcc.ch/organization, see also Bolin 2008). Its main product consists of assessment reports, published every 5-7 years, in three volumes from each of the three IPCC working groups. Working group one (WGI) covers the physical science basis, working group two (WGII) addresses impacts, adaptation and vulnerability at global and regional levels and working group three (WGIII) focusses on mitigation. IPCC also compiles a synthesis report providing a summary across those individual reports. The first IPCC report (FAR) was published in 1990 (complemented by a supplementary report in 1992) and followed by the second assessment report (SAR) in 1996, the third (TAR) in 2001, the fourth (AR4) in 2007 and the fifth and most recent (AR5) in 2013/2014.

Over the past decades, the possibility to perform increasingly complex, systematic numerical experiments has dramatically enhanced scientific understanding of fundamental properties of the Earth system and of the key drivers of climate change. The aspect of research concerned with the 
climate system and physical climate change that is based on general circulation models has developed virtually within and around the IPCC WGI. The community of climate modellers synchronizes the development of their model inter-comparison projects with the IPCC assessment cycles and use the platform and infrastructure provided by the IPCC for exchange and advancement of common standards and methodologies. The situation is different in WGII, which spans a very wide range of research disciplines and integrates local, regional and global drivers and conditions.

\subsubsection{IPCC confidence language}

The IPCC reports are science-policy assessments that integrate findings of varying degrees of uncertainty and communicate them to decision makers. The IPCC attaches qualifiers, such as 'very likely' or 'medium confidence' to key findings indicating their robustness. The confidence metric is briefly introduced here as it will be used in the assessment of the role of anthropogenic forcing for observed impacts in Chapter 5. For AR5, two metrics were used to communicate the degree of certainty (Mastrandrea et al 2010):

- Confidence in the validity of a finding, based on the type, amount, quality and consistency of evidence (e.g. mechanistic understanding, theory, data, models, expert judgement) and the degree of agreement, expressed qualitatively (see Figure 1.1); and

- Quantified measures of uncertainty in a finding expressed probabilistically (based on the statistical analysis of observations or model results, or expert judgement).

The WGII detection and attribution assessment applies the qualitative metric to express confidence in its findings, using the five qualifiers 'very low', 'low', 'medium', 'high' and 'very high'.
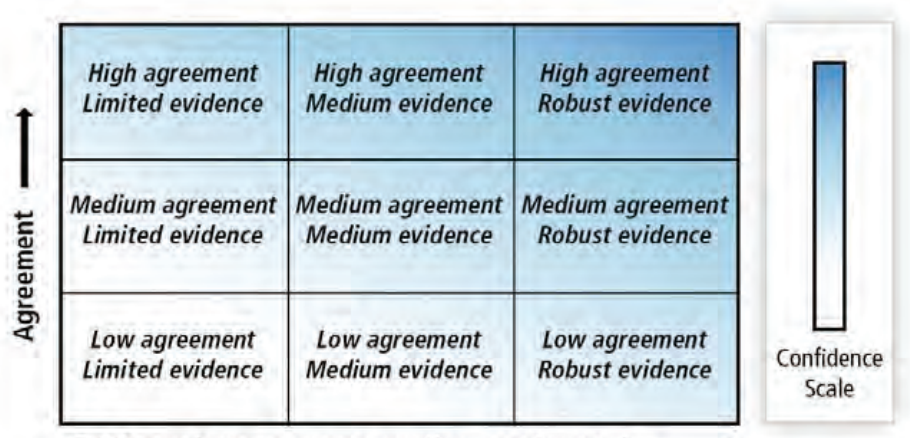

Evidence (type, amount, quality, consistency)

Figure 1.1: Schematic of evidence and agreement statements and their relationship to confidence. Confidence increases towards the top-right corner as suggested by the increasing strength of shading. Source: Figure 1.3 in IPCC (2014a). 


\subsubsection{Systems and drivers}

The terms 'climate system', 'natural and human systems' and 'physical, biological and human and managed systems' are used frequently in this thesis. A 'system' is confined by its boundaries and its behaviour results from internal dynamics (i.e. the interaction of components considered parts of the system) and external forcings or drivers that are outside of the system boundaries, but influence one or several of its components. For example, the climate system comprises the atmosphere, hydrosphere, biosphere, cryosphere and lithosphere. Humans and their activities constitute what is sometimes called the 'anthroposphere' (cf. Steffen et al 2011) and are generally considered external to the climate system (and most other natural systems). Human actions that lead to changes in atmospheric composition and land cover, such as the burning of fossil fuels and biomass, or deforestation, result in 'anthropogenic forcing' of the climate system (see Section 1.1.4), while solar variations and volcanoes are considered 'natural external forcing'. Both the terms 'driver' and 'forcing' are used in this thesis; to ensure clarity, the use of the word 'forcing' is limited to external forcings of the climate system, while in the context of impact systems, I refer to external and internal 'drivers'. Most impact systems are affected by more than one external driver. The most common external drivers that are important in the context of impact attribution are listed in Table

1.1. Note that many of the local drivers listed also have an indirect effect on the climate systems.

Table 1.1: Examples for relevant, non-climate drivers of change that directly or indirectly influence natural and human systems.

\begin{tabular}{|l|l|l|}
\hline Non-climate driver & Example & Examples of direct effects \\
\hline Land-use change & $\begin{array}{l}\text { Conversion of meadows to } \\
\text { croplands }\end{array}$ & $\begin{array}{l}\text { Biodiversity loss, altered water balance, change in habitat and } \\
\text { subsequent change in species composition, changes in soil } \\
\text { carbon and nutrient balance }\end{array}$ \\
\cline { 2 - 3 } & $\begin{array}{l}\text { Urbanisation and } \\
\text { Infrastructure development }\end{array}$ & $\begin{array}{l}\text { Habitat fragmentation, increased run-off and decrease in } \\
\text { groundwater renewal, increased disturbances (noise, light, } \\
\text { pollution) }\end{array}$ \\
\hline Land-cover change & $\begin{array}{l}\text { Desertification (through } \\
\text { overuse/degradation) }\end{array}$ & $\begin{array}{l}\text { Biodiversity loss, decrease in net primary productivity and } \\
\text { biomass, change in species composition, increase in dust load }\end{array}$ \\
\hline introduction & $\begin{array}{l}\text { Introduction of invasive mussel } \\
\text { species through ballast water }\end{array}$ & Changes in species composition, extinction of native species \\
\hline Population growth & $\begin{array}{l}\text { Pesticide and nutrient runoff } \\
\text { into rivers and lakes }\end{array}$ & $\begin{array}{l}\text { Algae blooms, hypoxic zones, increased stratification, change in } \\
\text { ecosystem composition }\end{array}$ \\
\cline { 2 - 3 } Increased NO input & $\begin{array}{l}\text { Fertilization effect on forests, change in soil dynamics } \\
\text { settlement in coastal areas }\end{array}$ & $\begin{array}{l}\text { Shoreline changes, degradation of coastal ecosystems, increased } \\
\text { wastewater input }\end{array}$ \\
\hline $\begin{array}{l}\text { Technological } \\
\text { change }\end{array}$ & $\begin{array}{l}\text { Agricultural intensification } \\
\text { (e.g. irrigation, fertilization, } \\
\text { mechanisation) }\end{array}$ & $\begin{array}{l}\text { Increase in yields, increase in water use, nutrient input and run- } \\
\text { off, change in composition of micro-organisms in soil and soil } \\
\text { water balances, change in agrobiodiversity, increase in labour } \\
\text { productivity, structural change }\end{array}$ \\
\hline Economic wealth & $\begin{array}{l}\text { Increased adoption of } \\
\text { residential air conditioning }\end{array}$ & $\begin{array}{l}\text { Increase in labour productivity and electricity consumption, } \\
\text { positive health effects, local pollution }\end{array}$ \\
\hline
\end{tabular}

System boundaries are usually ambiguous and subject to convention. The categorization of systems used in this thesis mirrors that of the IPCC AR5 WGII chapter on detection and attribution of 
observed impacts (Cramer et al 2014). For overview, the most important features of that characterization are summarized in Table 1.2. Also note that the term 'natural and human systems' is used synonymously with the term 'physical, biological and human and managed systems'. A 'managed system' usually refers to a managed ecosystem such as an agro-forestry plantation or a cultural landscape. However in some cases, for example the influence of reservoirs and barrages on a river, the 'management' refers mainly to the physical properties of the system. Categorization is arbitrary to a certain degree and disciplinary conventions vary. For example, coastal wetlands could be defined as terrestrial systems; wildfires are often considered part of the physical rather than the biological sphere (and hence part of the climate system); and many coastal effects will actually affect built infrastructure, i.e. a managed system.

Table 1.2: Overview of system definitions used in this thesis. Note that examples are not exhaustive. Systems, subsystems and categories correspond to those used by Cramer et al (2014).

\begin{tabular}{|c|c|c|c|}
\hline \multicolumn{2}{|c|}{ System } & Subsystem & Examples of impact categories \\
\hline \multirow{6}{*}{ 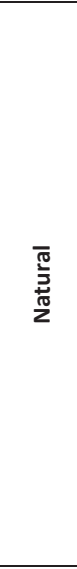 } & \multirow{3}{*}{ 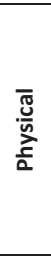 } & Cryosphere & $\begin{array}{l}\text { Glacier volume and length; lake and river ice; sea ice; lowland and } \\
\text { mountain permafrost; snow cover }\end{array}$ \\
\hline & & Hydrology & $\begin{array}{l}\text { Changes in pattern and magnitude of river run-off and river flow; lake } \\
\text { size and warming/stratification; soil moisture drought; floods; } \\
\text { groundwater change }\end{array}$ \\
\hline & & Coastal processes & $\begin{array}{l}\text { Coastal erosion and shoreline degradation; coastal flooding; } \\
\text { groundwater salinization; coastal permafrost degradation; changes in } \\
\text { wetlands and coastal ecosystem due to sea level rises }\end{array}$ \\
\hline & \multirow{3}{*}{$\frac{\overline{ }}{\frac{0}{00}}$} & Terrestrial ecosystems & $\begin{array}{l}\text { For terrestrial and freshwater systems: changes in species abundance, } \\
\text { composition, range or distribution, phenology and productivity; } \\
\text { synchronisation and ecosystem effects; extinctions; invasive species }\end{array}$ \\
\hline & & Marine ecosystems & $\begin{array}{l}\text { For marine ecosystems, including sea birds and sea-ice-based mammals, } \\
\text { and temperature effects on coastal ecosystems: changes in species } \\
\text { abundance, composition, range or distribution, phenology and } \\
\text { productivity; synchronisation and ecosystem effects; extinctions; } \\
\text { invasive species }\end{array}$ \\
\hline & & Wildfire & $\begin{array}{l}\text { Wildfire frequency; area burnt; intensity and duration of fires; } \\
\text { occurrence of mega fires; unprecedented wildfires }\end{array}$ \\
\hline \multirow{3}{*}{ 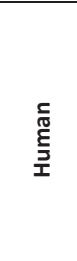 } & \multirow{3}{*}{ 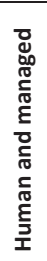 } & Food systems & $\begin{array}{l}\text { Cropping systems; agroforestry; livestock and pastoral systems; inland } \\
\text { and marine fisheries and aquaculture; horticulture; viticulture and } \\
\text { orchards }\end{array}$ \\
\hline & & Human health & $\begin{array}{l}\text { Heat and cold-related mortality; health effects of extreme heat; vector } \\
\text { borne diseases; water borne diseases }\end{array}$ \\
\hline & & $\begin{array}{l}\text { Livelihoods and other } \\
\text { socioeconomic indicators }\end{array}$ & $\begin{array}{l}\text { Livelihoods of rural populations, farmers, fishers and hunters; adaptive } \\
\text { responses; traditional and cultural practices and values; infrastructure } \\
\text { and travel; labour productivity }\end{array}$ \\
\hline
\end{tabular}

\subsubsection{Climate change}

This thesis follows the convention of the IPCC, using the term 'climate change' for any "change in the state of the climate that can be identified [...] by changes in the mean and/or the variability of its properties, and that persists for an extended period, typically decades or longer" (IPCC 2014b) and the term 'anthropogenic climate change' for a change of climate which is attributed directly or 
indirectly to human activity, i.e. anthropogenic forcing (see Table 1.3). Note that water vapour, an important contributor to the greenhouse effect, is considered internal to the climate system.

Table 1.3: Anthropogenic and natural external forcings of the climate system.

\begin{tabular}{|c|c|c|}
\hline \multicolumn{2}{|l|}{ Anthropogenic forcing } & \multirow[t]{2}{*}{ Natural forcing } \\
\hline $\begin{array}{l}\text { Anthropogenic emission of greenhouse gases } \\
\text { and other forcing agents }\end{array}$ & Land-use change & \\
\hline $\begin{array}{l}\text { Change in the atmospheric composition of carbon dioxide }\left(\mathrm{CO}_{2}\right) \text {, } \\
\text { nitrous oxide }\left(\mathrm{N}_{2} \mathrm{O}\right) \text {, methane }\left(\mathrm{CH}_{4}\right) \text {, Ozone }\left(\mathrm{O}_{3}\right) \text {, sulphur } \\
\text { hexafluoride }\left(\mathrm{SF}_{6}\right) \text { and many halogenated species; primary aerosols } \\
\text { (black carbon, organic carbon) and secondary aerosols (sulphate, } \\
\text { nitrate, ammonium and secondary organic aerosols) }\end{array}$ & $\begin{array}{l}\text { Albedo effects } \\
\text { Changes in the atmospheric } \\
\text { composition (carbon budget } \\
\text { and balance, aerosol } \\
\text { emissions) }\end{array}$ & $\begin{array}{l}\text { Solar variations, } \\
\text { Volcanic activity }\end{array}$ \\
\hline
\end{tabular}

In the context of climate attribution, the terms 'anthropogenic forcing', 'human activity' and 'human influence' are often used synonymously; though in this thesis, 'anthropogenic forcing' is preferred to increase clarity. Anthropogenic forcing includes the direct emission of greenhouse gases, the change of Albedo through large-scale land-use change and human induced changes in the carbon cycle, i.e. all effects that are generally represented by global climate models (Taylor et al 2012).

\subsection{Detection and attribution}

Within the context of climate change research, concepts of detection and attribution refer mostly to the detection of changes in the climate system and their attribution to anthropogenic forcing (Zwiers and Hegerl 2008; Hegerl and Zwiers 2011), including the occurrence of extreme weather (Stott et al 2013). The difficulty in providing 'proof' that the climate is really changing due to human influence constitutes an early and central problem for climate change research. The main challenge consists in isolating a coherent signal due to anthropogenic forcing from the background 'noise' of natural climate variability, including inter-annual and inter-decadal variations caused by large-scale modes such as El Niño Southern Oscillation (ENSO) or the Indian Ocean Dipole. Work on the statistical properties of climate model simulations to tackle this 'signal-to-noise' problem dates back to the 1970s (Chervin et al 1974; Chervin and Schneider 1976; Thompson and Schneider 1982). Approaches to enhancing the signal-to-noise ratio by averaging across ensembles of climate model simulations and across space and time still form the basis of what has evolved to become climate change 'detection and attribution' (see Barnett et al 1999; Hegerl et al 2007; Bindoff et al 2013).

\subsection{1 'Detection and attribution' - genesis of terminology}

As could be witnessed during the government and expert review of the IPCC's AR5, parts of the research community and many stakeholders understand 'attribution' to stand synonymously for 'attribution of a change to anthropogenic forcing of the climate system'. However, in literal terms, neither detection nor attribution are connected to a specific system or end point (cause), but 
describe the establishment of a fact and the examination of causes or contributors to that fact (see

Table 1.4). Similarly, no universal method exists for inferring conclusions about cause and effect, though the definition of detection in a statistical sense is clear.

Table 1.4: Literal definitions of detection and attribution and historic development of definitions used by the IPCC. Adapted from Tables 1 and 2 in supplementary material to Stone et al (2013).

\begin{tabular}{|c|c|c|}
\hline Source & Definition of detection & Definition of attribution \\
\hline $\begin{array}{l}\text { Merriam- } \\
\text { Webster } \\
\text { online } \\
\text { dictionary }\end{array}$ & $\begin{array}{l}\text { Detection: the act of detecting; the state or fact of being } \\
\text { detected. } \\
\text { To detect: to discover the true character of; to discover } \\
\text { or determine the existence, presence, or fact of. }\end{array}$ & $\begin{array}{l}\text { Attribution: the act of attributing. } \\
\text { To attribute: to explain by indicating a cause. }\end{array}$ \\
\hline $\begin{array}{l}\text { IPCC FAR WGI } \\
\text { (Wigley et al } \\
\text { 1990) }\end{array}$ & $\begin{array}{l}\text { The word detection has been used to refer to the } \\
\text { identification of a significant change in climate. ... To } \\
\text { claim detection in a useful and practical way, we must } \\
\text { not only identify a climatic change, but we must } \\
\text { attribute at least part of such a chance to the enhanced } \\
\text { greenhouse effect ... Detection requires that the } \\
\text { observed changes in climate are in accord with detailed } \\
\text { model predictions of the enhanced greenhouse effect. }\end{array}$ & not defined (considered part of detection) \\
\hline $\begin{array}{l}\text { IPCC SAR WGI } \\
\text { (Santer et al } \\
\text { 1996) }\end{array}$ & $\begin{array}{l}\text { Detection of change is the process of demonstrating } \\
\text { that an observed change in climate is highly unusual in a } \\
\text { statistical sense, but does not provide a reason for the } \\
\text { change. }\end{array}$ & $\begin{array}{l}\text { Attribution is the process of establishing cause } \\
\text { and effect, i.e. that changes in anthropogenic } \\
\text { emissions are required in order to explain } \\
\text { satisfactorily the observed change in climate. }\end{array}$ \\
\hline $\begin{array}{l}\text { IPCC TAR WGI } \\
\text { (Mitchell et al } \\
\text { 2001) }\end{array}$ & \multicolumn{2}{|c|}{$\begin{array}{l}\text { Detection and attribution of climate change to anthropogenic causes (i.e. the isolation of cause and } \\
\text { effect) ... involves statistical analysis and the careful assessment of multiple lines of evidence to } \\
\text { demonstrate, within a pre-specified margin of error, that the observed changes are: unlikely to be due } \\
\text { entirely to internal variability; consistent with the estimated responses to the given combination of } \\
\text { anthropogenic and natural forcing; and not consistent with alternative, physically plausible explanations } \\
\text { of recent climate change that exclude important elements of the given combination of forcings. }\end{array}$} \\
\hline $\begin{array}{l}\text { IPCC TAR } \\
\text { WGII (Ahmad } \\
\text { et al 2001) }\end{array}$ & $\begin{array}{l}\text { Assessment of the impacts on human and natural } \\
\text { systems that already have occurred as a result of recent } \\
\text { climate change. [...] An important component of the } \\
\text { detection process is the search for systematic patterns } \\
\text { of change across many studies that are consistent with } \\
\text { expectations. }\end{array}$ & $\begin{array}{l}\text { Attribution of observed changes in natural } \\
\text { systems to the effects of climate change is } \\
\text { analogous to attribution of anthropogenic } \\
\text { greenhouse gases as causal factors of recent } \\
\text { climate trends. }\end{array}$ \\
\hline $\begin{array}{l}\text { IPCC AR4 WGI } \\
\text { (Hegerl et al } \\
2007 \text { ) }\end{array}$ & $\begin{array}{l}\text { Detection is the process of demonstrating that climate } \\
\text { has changed in some defined statistical sense, without } \\
\text { providing a reason for that change. ... An identified } \\
\text { change is detected in observations if its likelihood of } \\
\text { occurrence by chance due to internal variability alone is } \\
\text { determined to be small. }\end{array}$ & $\begin{array}{l}\text { Attribution of causes of climate change is the } \\
\text { process of establishing the most likely causes } \\
\text { for the detected change with some defined } \\
\text { level of confidence. }\end{array}$ \\
\hline $\begin{array}{l}\text { IPCC AR4 } \\
\text { WGII } \\
\text { (Rosenzweig } \\
\text { et al 2007) }\end{array}$ & \multicolumn{2}{|c|}{$\begin{array}{l}\text { Detection and attribution of observed changes and responses in systems to anthropogenic forcing is } \\
\text { usually a two-stage process. First, the observed changes in a system must be demonstrated to be } \\
\text { associated with an observed regional climate change within a specified degree of confidence. Second, a } \\
\text { measurable portion of the observed regional climate change, or the associated observed change in the } \\
\text { system, must be attributed to anthropogenic causes with a similar degree of confidence. }\end{array}$} \\
\hline $\begin{array}{l}\text { IPCC good } \\
\text { practice } \\
\text { guidance; } \\
\text { (Hegerl et al } \\
\text { 2010) } \\
\text { IPCC AR5 } \\
\text { Glossary }\end{array}$ & $\begin{array}{l}\text { Detection of change is defined as the process of } \\
\text { demonstrating that climate or a system affected by } \\
\text { climate has changed in some defined statistical sense } \\
\text { without providing a reason for that change. An } \\
\text { identified change is detected in observations if its } \\
\text { likelihood of occurrence by chance due to internal } \\
\text { variability alone is determined to be small. }\end{array}$ & $\begin{array}{l}\text { Attribution is defined as the process of } \\
\text { evaluating the relative contributions of } \\
\text { multiple causal factors to a change or event } \\
\text { with an assignment of statistical confidence. }\end{array}$ \\
\hline $\begin{array}{l}\text { IPCC AR5 } \\
\text { Glossary } \\
\text { (IPCC } 2014 b)\end{array}$ & \multicolumn{2}{|c|}{$\begin{array}{l}\text { Detection of impacts of climate change. For a natural, human, or managed system, identification of a } \\
\text { change from a specified baseline. The baseline characterizes behaviour in the absence of climate change } \\
\text { and may be stationary or non-stationary (e.g. due to land-use change). }\end{array}$} \\
\hline
\end{tabular}


The definition of what constitutes detection and attribution differs between assessment cycles and also between working groups (see Table 1.4). In the first assessment report by WGI, attribution is considered part of detection, while later definitions separate the two steps of determining a statistical significant change beyond natural variability (detection) and the examination of the causes contributing to that change (attribution).

In the context of the impact of past climate change, a focus on observed effects of climate change emerged only in the Third Assessment Report. The technical summary of the WGII Second Assessment Report states: "Unambiguous detection of climate-induced changes in most ecological and social systems will prove extremely difficult in the coming decades. This is because of the complexity of these systems, their many non-linear feedbacks and their sensitivity to a large number of climatic and non-climatic factors, all of which are expected to continue to change simultaneously" (IPCC 1996). Though this quote relates to both past and future impacts of climate change, it reveals the fundamental challenge regarding attribution of impacts - disentangling the parallel and interacting influences of multiple local and global change drivers.

\subsubsection{Detection and attribution: from climate to impacts}

The attribution of impacts to climate change (hereinafter 'impact attribution') differs from attribution of climate change to anthropogenic forcing of the climate system (hereinafter 'climate attribution') in many ways. The most fundamental issues concern the end point of the analysis (climate change vs. anthropogenic forcing) and the consequences of the multifactorial nature of most impact systems for the behaviour of the system in the absence of (anthropogenic) climate change.

Studies that attribute observed impacts in natural and human systems to anthropogenic forcing are still very rare (e.g. Barnett et al 2008; Christidis et al 2010a; Marzeion et al 2014). In the field of ecology, synthesis assessments of large datasets of long-term local observations have been used to identify 'fingerprints' of anthropogenic forcing (Parmesan and Yohe 2003; Root et al 2005; Rosenzweig et al 2008; Poloczanska et al 2013). The end point of an impact attribution study can be either local or regional trends in climate, or anthropogenic forcing of the climate system. The majority of observational impact studies examine the effects of recent changes in climate.

The central challenge for the climate science community consists of filtering a climate change signal from the noise created by internal climate variability in both model results and observational data (see Section 1.4), while the properties, internal relationships and external drivers of the climate system are well understood on global scales. This is a fundamentally different situation from many natural and human systems, whereby several global (and local) change drivers are seen to have a 
simultaneous impact on systems and neither those processes nor their interactions are necessarily understood well enough to isolate the influence of one factor from the other. As a consequence, such systems are difficult to describe in terms of 'internal variability'.

The definitions provided in the IPCC's good practice guidance paper have therefore been further specified for use in the context of climate change impacts by Stone et al (2013) and applied in the WGII AR5. In particular, Stone et al (2013) elaborate on the need to establish clear baselines during the detection phase and suggest ascribing a magnitude to the attribution statement, i.e. whether climate change has a 'major' or 'minor' role in the observed change. They argue that detection and attribution cannot be separated entirely, as detection must involve controlling for the effect of other drivers. Their approach emphasizes the role of climate change compared to other factors and implies that part of what is considered the attribution phase should be done during the impact detection phase.

Attribution is defined as the process of evaluating the relative contributions of multiple causal factors to a change or event with an assignment of statistical confidence (Hegerl et al 2010). Impact detection as defined above implies that climate change has played at least a minor (i.e. statistically significantly different from zero) role in the observed system behaviour. Detection means rejecting the null hypothesis of no climate change effect. The result of a detection analysis is therefore binary: an effect has or has not been detected. Attribution follows detection by assessing the magnitude of the observed effect. Note that the latter is an estimation and not hypothesis testing; its results should therefore be expressed by a quantifier.

WGI type attribution assessments often refer to anthropogenic forcing explaining 'most of' or 'at least half of' the observed effect (e.g. Bindoff et al 2013). This is in keeping with the earlier definition of attribution as "the process of establishing the most likely causes for a detected change with some level of confidence" (Stott et al 2010). In the context of impacts, attribution addresses the magnitude of the contribution of climate change to a change in a system (Cramer et al 2014), however that magnitude can be small compared to other factors. With a WGI type definition of 'most likely cause' in mind, the impact attribution findings of Cramer et al (2014) may cause confusion. For example, the dominant cause for observed increases in coastal erosion at a specific location might be reduced sediment supply and beach mining. However, this does not imply that climate change, i.e. rising sea levels due to thermal expansion and increased glacial melt and run-off, does not contribute to the observed effects at all. The explicit formulation of relative roles in Cramer et al (2014) accounts for such complexity. In essence, many attribution statements in IPCC WGII AR5 are 'partial attribution' statements. 


\subsubsection{Good practice guidance paper on detection and attribution}

Recognizing the differences in concepts and approaches between working groups, the IPCC hosted an expert meeting on "detection and attribution related to anthropogenic climate change" in September 2009. As an outcome of that meeting, the "good practice guidance paper on detection and attribution related to anthropogenic climate change" was drafted (Hegerl et al 2010), intended for the use of the authors of WGI and WGII during the fifth assessment cycle. This section summarizes some key elements of that document.

The good practice guidance paper contains a set of definitions for relevant terms such as detection, attribution (see Table 1.4), confounding factor and external driver/forcing. It also discusses data and other requirements and the handling of external drivers/forcing and confounding factors. A set of four attribution methods is specified, which are briefly sketched below (for details, see Stone et al 2009; Hegerl et al 2010). Determining the level of confidence in discontinuous or joint assessments presents a key challenge. The IPCC AR4 glossary clearly states, "Confidence in such joint attribution statements must be lower than the confidence in either of the individual attribution steps alone due to the combination of two separate statistical assessments" (IPCC 2007a). However, this is probably practically not as straightforward as indicated, given that the nature of the various steps of the assessments may be different and possibly not quantitative.

Although the last of the four methods described in the good practice guidance paper concerns the influence of all climatic changes (regardless of their cause) on natural and human systems, the guidance paper primarily focusses on establishing a link between anthropogenic forcing and observed impacts. This fact is also made apparent in the disciplinary composition of the meeting participants and the subjects covered in the meeting report (IPCC 2010). A group of WGII authors later criticized what they perceived to be the IPCC's focus on the anthropogenic component of climate change, raising doubts about both the feasibility and usefulness of such an approach in the context of ecology (Parmesan et al 2011). Their comments were refuted by several other researchers involved in that meeting (Brander et al 2011; Hoegh-Guldberg et al 2011; Stocker et al 2011). However, controversy still persists as to whether the evaluation of the contribution of anthropogenic forcing to observed trends in impact systems should be a priority for research (Parmesan et al 2013).

\subsubsection{Single step attribution to acts of external forcing}

As the title suggests, the single step attribution method is based on the explicit modelling of a variable's response to external forcing by either a single comprehensive model set-up, or by a sequential analysis using several models, whereby the output of one modelling step provides the 
input for the next step (see Figure 1.2). Other authors have used the term 'end-to-end analysis' to differentiate this approach from ones that rely on sequential analysis, but have a discontinuity between the different steps (see Section 1.2.3.2).

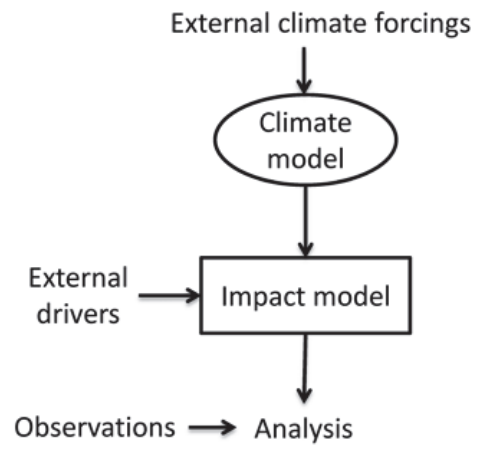

Figure 1.2: Schematic of an end-to-end analysis, based on Stone et al (2009). External drivers refer to nonclimatic drivers of change that influence the impact system.

The few existing applications usually estimate whether observed trends in a non-climate system appear inconsistent with expected behaviour in a stationary climate and consistent with what could be expected from externally forced climate change. In essence, these studies provide a comparison of likelihood estimates, without considering local drivers of change. For example, Gillett et al (2004) detect an anthropogenic forcing signal in areas of forest burnt in Canada based on a single-step model. Note that following the approach of AR5 WGII, this study would be classified as a detection study, as it does not provide any insights regarding the relative role of anthropogenic climate change beyond the fact that it is 'detectable'.

\subsubsection{Multi-step attribution to external forcing}

The multi-step attribution method consists of the combination of findings for the individual steps of an impact attribution exercise (observation of a change; association of that change with a climate trend; and association of the climate trend to anthropogenic forcing) from different sources (see Figure 1.3). To emphasize the discontinuity between the different steps, this method has also been dubbed sequential analysis. The sequence of analysis must be followed by an explicit synthesis of the individual steps and confidence in the overall finding will typically be similar to, or lower than, the weakest confidence for any individual step. 


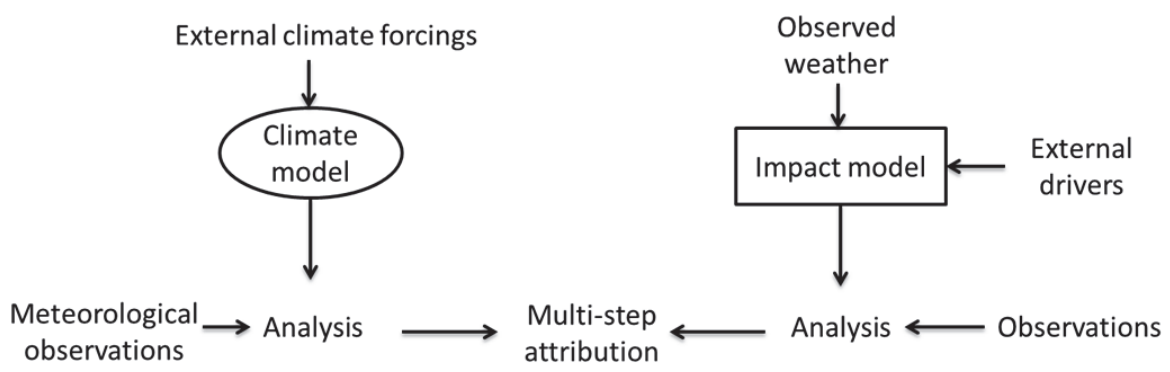

Figure 1.3: Schematic of sequential or multi-step attribution analysis, based on Stone et al (2009). External drivers refer to non-climatic drivers of change that influence the impact system.

\subsubsection{Associative pattern attribution to external forcings}

Multiple analyses of climate-impact systems are synthesized and the ensemble of results is linked to anthropogenic forcing by statistical pattern comparison measures. This approach was first used by Rosenzweig et al (2007) in AR4 and carried forward to follow-up publications (Rosenzweig et al 2008; Rosenzweig and Neofotis 2013). Associative pattern attribution is a realization of what Stone et al (2014) call 'synthesis analysis', where the spatial pattern of change derived from multiple analyses of individual observed changes are compared to the patterns that would be expected to emerge based on observed climate change and the observed changes in climate are then attributed to anthropogenic forcing by a separate climate analysis.

\subsubsection{Attribution to a change in climatic conditions}

Attribution to a change in climatic conditions is a variation of the multi-step method introduced in Section 1.2.3.2. It assesses the role of changing climatic conditions in the overall change of the relevant impact system, compared to other drivers of change. Such an assessment is always based on process understanding; it often involves quantitative analysis of observational data with statistical models and conclusions derived from numerical process models and field experiments (see also Section 1.3).

\subsection{Observed impacts of climate change}

The effects of climate change on natural and human systems are now evident across continents and oceans for both of these systems (Rosenzweig and Neofotis 2013; Cramer et al 2014). Two decades ago, robust evidence for observed climate change impacts was almost exclusively available for the cryosphere (WGMS 1989; Fitzharris et al 1995) and some terrestrial ecosystems in mid to high northern latitudes (Grabherr et al 1994; Myneni et al 1997). Over the last decade, evidence has increased especially for the impact on marine ecosystems, food production and wildfire regimes. 
Evidence linking observed impacts to changes in precipitation is increasingly available, however, there continues to be a gap in the documentation of the effects of changing sea levels due to climate change. Also, despite improvements in the evidence base for southern regions, the global distribution of observed impacts remains uneven. Figure 1.4 shows the graphical representations of observed climate change impacts from IPCC's WGII TAR and AR4 summaries for policymakers, and Figure 1.5 shows the corresponding AR5 summary figure from Cramer et al (2014).

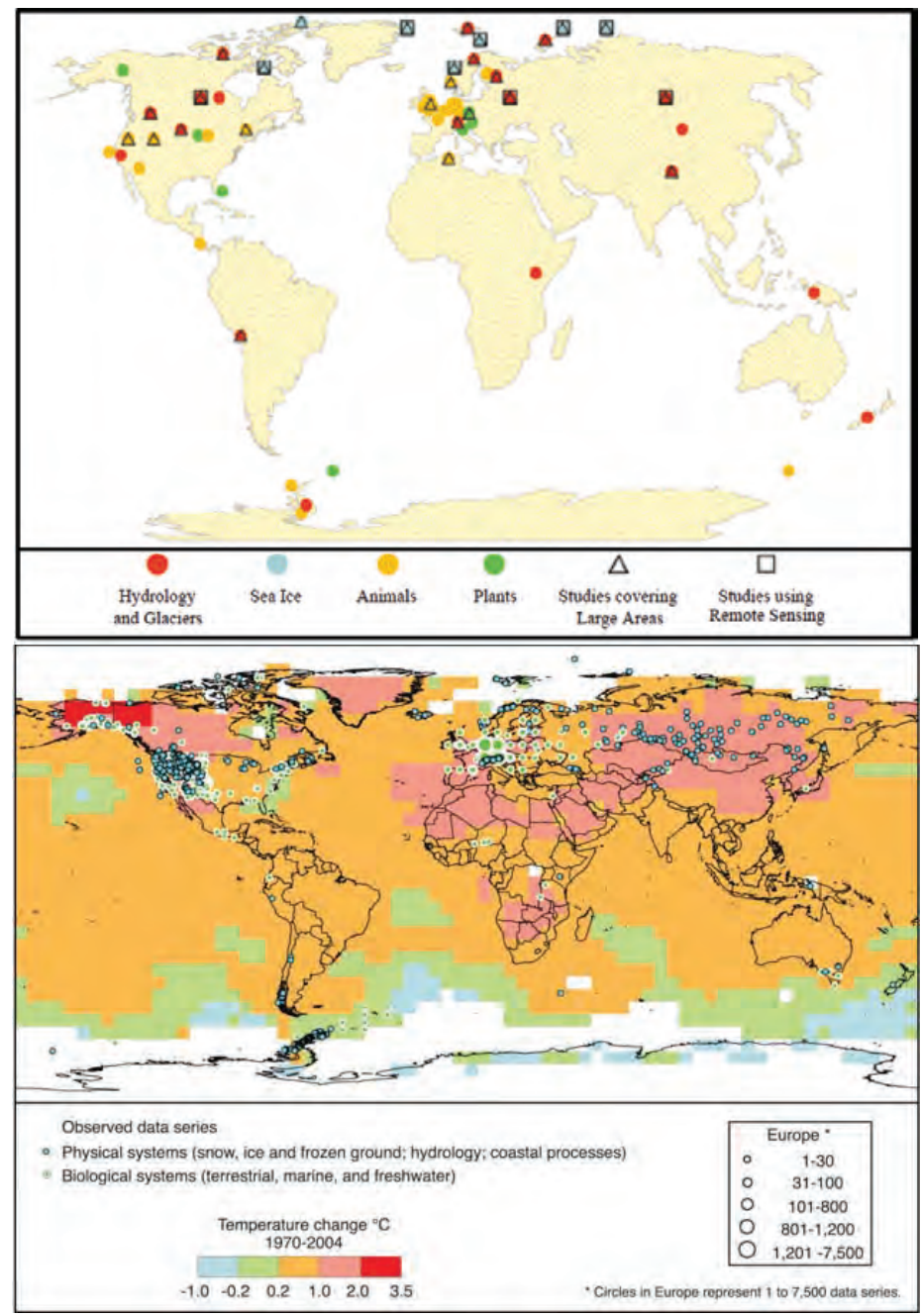

Figure 1.4: Observed impacts of climate change, as represented in the summary for policymakers of the third (upper panel) and the fourth (bottom panel) IPCC assessment report respectively. Icons on the TAR map depict one or several studies on the same subject. Circles on the AR4 map represent time series of georeferenced data, with circle sizes representing the number of data series, and circle colour differentiating between physical and biological systems. The background colour indicates observed temperature change in the respective grid cell. All observations relate to temperature change. Source: IPCC (2001), IPCC (2007b). 


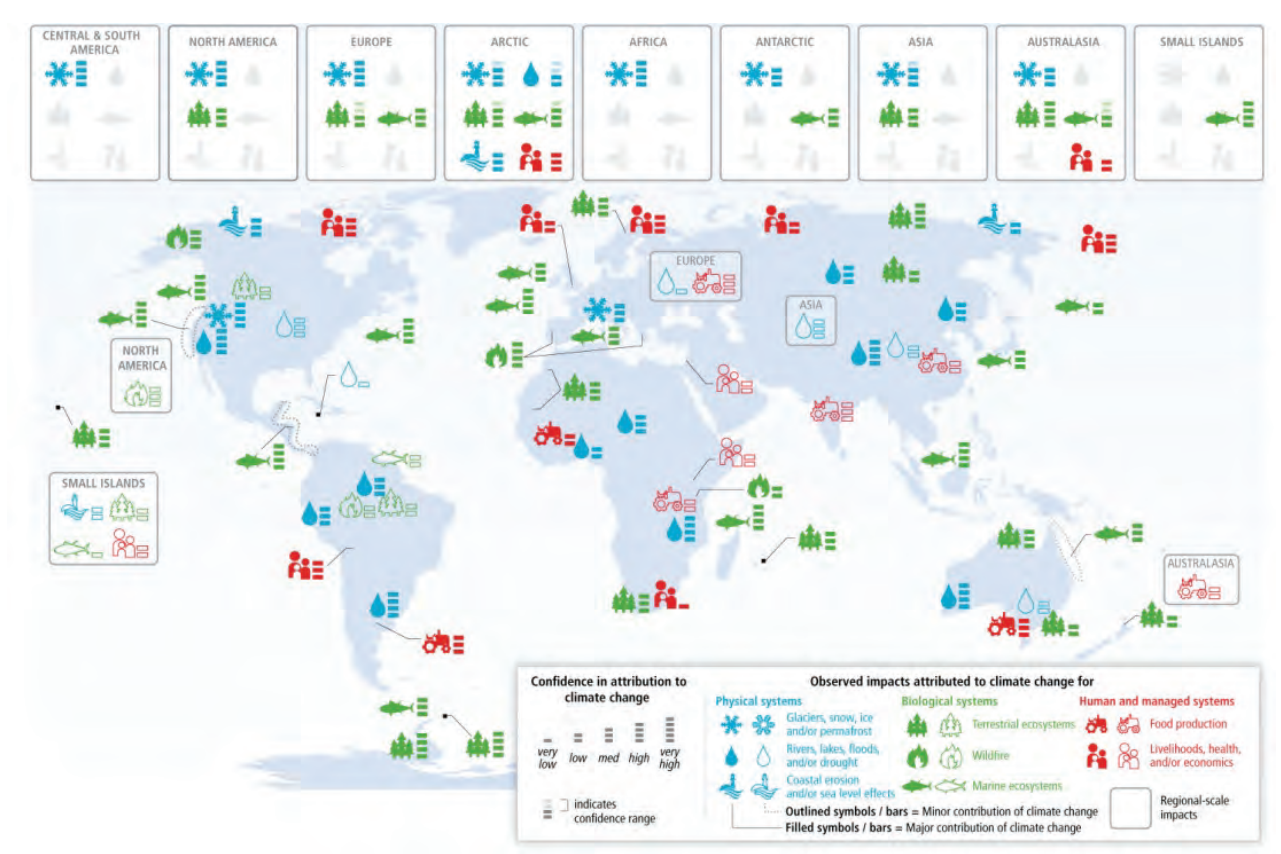

Figure 1.5: Observed impacts of climate change as represented in IPCC's WGII AR5, Cramer et al (2014). Icons on the map represent subcontinental assessments of different scope for one of nine overarching impact categories. Icons in the boxes above represent systems for which at least one impact within a category has been found to apply across the whole region. The bars show the corresponding confidence in attribution of the effect to climate change. Source: Figure 18.3 in Cramer et al (2014).

Although the methods applied, the definitions of regions and the scope of analyses differ to a certain degree between the three assessment cycles, the three graphics displayed in Figures 1.4 and 1.5 give a good indication for the development of evidence over the last two decades.

IPCC's AR4 also links the observed effects of regional climate changes to anthropogenic forcing of the climate system (IPCC 2007b; Rosenzweig et al 2007), concluding that "... it is likely that anthropogenic warming has had a discernible influence on many physical and biological systems." For AR5, neither the WGII contribution nor the synthesis report provided an explicit link between the observed changes in natural and human systems and the anthropogenic component of regional climate changes. One of the objectives of this thesis is to close this gap.

\subsection{Attribution of observed climate change to human influence}

The detection and attribution of changes in global and regional climate to anthropogenic forcing is discussed within IPCC WGI, Chapter 10 (Bindoff et al 2013). However, Bindoff et al mainly address global issues. Some more regional detail, in particular with regard to observations of extreme weather, can be found in the IPCC special report on managing the risk of extreme events and 
disasters to advance climate change adaptation (SREX, IPCC 2012), specifically in their Chapters 3 and 4 (Handmer et al 2012; Seneviratne et al 2012). Individual sets of detection and attribution information are available for a variety of regions, domain sizes, seasons and/or periods (e.g. Karoly and Wu 2005; Jones et al 2013).

A full review of all available literature on climate detection and attribution is beyond the scope of this introduction. To provide some context, the main findings from the latest IPCC WGI report are summarized below for climate averages (Section 1.4.1) and climate variability (Section 1.4.2). The following subsections mostly consist of statements copied verbatim from the summary for policymakers [SPM], technical summary [TS] or the 'frequently asked questions' [FAQ] of the WGI contribution to the IPCC AR5 (IPCC 2013b); these are labelled as italics and references are given in square brackets at the end of each paragraph.

\subsubsection{Observed changes in climate averages}

Below, the summarized findings of WGI concerning detection and attribution of changes in global mean temperature, precipitation, sea level, sea ice extent and chemical ocean properties are listed.

\subsubsection{Temperature}

It is extremely likely that more than half of the observed increase in global average surface temperature from 1951 to 2010 was caused by the anthropogenic increase in greenhouse gas concentrations and other anthropogenic forcings together. The best estimate of the human-induced contribution to warming is similar to the observed warming over this period. Over every continental region except Antarctica, anthropogenic forcings have likely made a substantial contribution to surface temperature increases since the mid-20th century. For Antarctica, large observational uncertainties result in low confidence that anthropogenic forcings have contributed to the observed warming averaged over available stations. It is likely that there has been an anthropogenic contribution to the very substantial Arctic warming since the mid-20th century. It is very likely that anthropogenic forcings have made a substantial contribution to increases in global upper ocean heat content $(0-700 \mathrm{~m})$ observed since the 1970s. There is evidence for human influence in some individual ocean basins. [SPM D.3]

\subsubsection{Precipitation}

It is likely that anthropogenic influences have affected the global water cycle since 1960. Anthropogenic influences have contributed to observed increases in atmospheric moisture content in the atmosphere (medium confidence), to global-scale changes in precipitation patterns over land (medium confidence), to intensification of heavy precipitation over land regions where data are 
sufficient (medium confidence), and to changes in surface and sub-surface ocean salinity (very likely). [SPM D.3]

\subsubsection{Sea level}

It is very likely that there is a substantial anthropogenic contribution to the global mean sea level rise since the 1970s. This is based on the high confidence in an anthropogenic influence on the two largest contributions to sea level rise, that is, thermal expansion and glacier mass loss. [SPM D.3]

\subsubsection{Sea ice}

Anthropogenic influences have very likely contributed to Arctic sea ice loss since 1979. There is low confidence in the scientific understanding of the small observed increase in Antarctic sea ice extent due to the incomplete and competing scientific explanations for the causes of change and low confidence in estimates of natural internal variability in that region. [SPM D.3]

\subsubsection{Chemical properties of the ocean}

Global analyses of oxygen data from the 1960s to 1990s extend the spatial coverage from local to global scales and have been used in attribution studies with output from a limited range of Earth System Models (ESMs). It is concluded that there is medium confidence that the observed global pattern of decrease in dissolved oxygen in the oceans can be attributed in part to human influences

The observations show distinct trends for ocean acidification (which is observed to be between 0.0014 and $-0.0024 \mathrm{pH}$ units per year). There is high confidence that the $\mathrm{pH}$ of ocean surface seawater decreased by about 0.1 since the beginning of the industrial era as a consequence of the oceanic uptake of anthropogenic $\mathrm{CO}_{2}$. [TS 4.4]

\subsubsection{Observed changes in climate variability}

Negative consequences of climate change are expected to unfold through increases in the frequency or intensity of weather and climate extremes. For moderate extremes, i.e. those occurring fairly regularly, the observational basis for detecting and attributing changes is sufficient for detection and attribution analysis in many regions. The following sections briefly summarize the status of knowledge concerning observed changes in extreme weather (Section 1.4.2.1, Table 1.5) and give a short overview about recent developments in the field of event attribution (Section 1.4.2.2)

\subsubsection{Trends in observed frequency and/or intensity of extreme weather}

There has been further strengthening of the evidence for human influence on temperature extremes since the SREX. It is now very likely that human influence has contributed to observed global scale 
changes in the frequency and intensity of daily temperature extremes since the mid-20th century, and likely that human influence has more than doubled the probability of occurrence of heat waves in some locations [SPM D.3]

Table 1.5: Changes in frequency and intensity of extreme weather events and confidence in a human contribution to that observed change, based on IPCC AR5 WGI Table SPM.1 (IPCC 2013a). Statements in brackets refer to assessment in IPCC's special report on managing the risk of extreme events and disasters to advance climate change adaptation (IPCC 2012) and WGI AR4 (IPCC 2007c) respectively.

\begin{tabular}{|c|c|c|}
\hline $\begin{array}{l}\text { Phenomenon and direction of } \\
\text { trend }\end{array}$ & $\begin{array}{l}\text { Assessment that changes have occurred } \\
\text { (since 1950, unless otherwise indicated) }\end{array}$ & $\begin{array}{l}\text { Assessment of a human contribution } \\
\text { to observed changes }\end{array}$ \\
\hline $\begin{array}{l}\text { Warmer and/or fewer cold days } \\
\text { and nights over most land areas }\end{array}$ & Very likely (very likely / very likely) & Very likely (likely/likely) \\
\hline $\begin{array}{l}\text { Warmer and/or more frequent } \\
\text { hot days and nights over most } \\
\text { land areas }\end{array}$ & Very likely (very likely / very likely) & Very likely (likely/likely (nights only)) \\
\hline $\begin{array}{l}\text { Warm spells/heat waves: } \\
\text { frequency and or duration } \\
\text { increase over most land areas }\end{array}$ & $\begin{array}{l}\text { Medium confidence globally; likely in large } \\
\text { parts of Europe, Asia and Australia } \\
\text { (medium confidence in many areas; likely) }\end{array}$ & Likely (N.A./more likely than not) \\
\hline $\begin{array}{l}\text { Heavy precipitation events } \\
\text { Increase in the frequency and/or } \\
\text { duration of heavy precipitation }\end{array}$ & $\begin{array}{l}\text { Likely more land areas with increases than } \\
\text { decreases ( } \sim \text { likely over most land areas) }\end{array}$ & $\begin{array}{l}\text { Medium confidence (medium } \\
\text { confidence/more likely than not) }\end{array}$ \\
\hline $\begin{array}{l}\text { Increase in intensity or duration } \\
\text { of drought }\end{array}$ & $\begin{array}{l}\text { Low confidence globally, likely changes in } \\
\text { some regions (likely in some regions/likely } \\
\text { in many regions since 1970) }\end{array}$ & $\begin{array}{l}\text { Low confidence (medium confidence/ } \\
\text { more likely than not) }\end{array}$ \\
\hline $\begin{array}{l}\text { Increases in intense tropical } \\
\text { cyclone activity }\end{array}$ & $\begin{array}{l}\text { Low confidence in long-term (centennial) } \\
\text { change, virtually certain in North Atlantic } \\
\text { since } 1970 \text { (low confidence/likely in some } \\
\text { regions since 1970) }\end{array}$ & $\begin{array}{l}\text { Low confidence (low confidence/more } \\
\text { likely than not) }\end{array}$ \\
\hline $\begin{array}{l}\text { Increased incidence or magnitude } \\
\text { of extreme high sea level }\end{array}$ & $\begin{array}{lllll}\begin{array}{l}\text { Likely (since 1970) } \\
\text { century/likely) }\end{array} & \text { (likely } & \text {-late } & 20^{\text {th }} \\
\end{array}$ & Likely (likely/more likely than not) \\
\hline
\end{tabular}

Changes in extremes for other climate variables are generally less coherent than those observed for temperature, owing to data limitations and inconsistencies between studies, regions and/or seasons. However, increases in precipitation extremes, for example, are consistent with a warmer climate. Analyses of land areas with sufficient data indicate increases in the frequency and intensity of extreme precipitation events in recent decades, but results vary strongly between regions and seasons. For instance, evidence is most compelling for increases in heavy precipitation in North America, Central America and Europe, but in some other regions-such as southern Australia and western Asia-there is evidence of decreases. Likewise, drought studies do not agree on the sign of the global trend, with regional inconsistencies in trends also dependent on how droughts are defined. However, indications exist that droughts have increased in some regions (e.g. the Mediterranean) and decreased in others (e.g. central North America) since the middle of the 20th century.

Considering other extremes, such as tropical cyclones, the latest assessments show that due to problems with past observing capabilities, it is difficult to make conclusive statements about long- 
term trends. There is very strong evidence, however, that storm activity has increased in the North Atlantic since the 1970s. (WGI FAQ 2.2)

Although the AR4 concluded that it is more likely than not that anthropogenic influence has contributed to an increased risk of drought in the second half of the 20th century, an updated assessment of the observational evidence indicates that the AR4 conclusions regarding global increasing trends in hydrological droughts since the 1970s are no longer supported. Owing to the low confidence in observed large-scale trends in dryness combined with difficulties in distinguishing decadal-scale variability in drought from long-term climate change, there is now low confidence in the attribution of changes in drought over global land since the mid-20th century to human influence. [TS4.7]

For an extended discussion of the attribution of extreme events to anthropogenic forcing of the climate system, see also Section 10.6 in Bindoff et al (2013).

\subsubsection{Attributing individual events and their consequences to human influence}

Given the potential for high costs and adverse consequences associated with extreme weather, a growing body of literature examines the role of anthropogenic forcing in recent extreme events (Peterson et al 2013b; Herring et al 2014). Trends in frequency and occurrence of some forms of extreme weather have been observed and attributed to anthropogenic forcing (Table 1.5). However, attributing the impact of individual extreme events to human influence on the climate system is an entirely different exercise. For one thing, the impact of an individual event depends on many factors that determine exposure and vulnerability of ecosystems and societies to such a type of event in general, but also on the specific conditions under which the event occurred (Allen et al 2007; Handmer et al 2012; Huggel et al 2013). Then again, due to the stochastic nature of weather, it has long been held that individual events cannot be attributed to anthropogenic climate change (Stott et al 2013). However, a consensus is now emerging that probabilistic approaches can be used to quantify the role of external (anthropogenic) drivers in specific events (Bindoff et al 2013).

IPCC'S AR5 describes two types of approaches to event attribution in the literature: "attributable magnitude" and "attributable risk" (Bindoff et al 2013, 10.6.). In general, such studies attempt to estimate the unusualness of a certain event under historic natural climate conditions and in a changed climate. Most publications focus on a variation of the "fraction of attributable risk" (Stone and Allen 2005), as a measure of the increase in probability of an event occurring that is of a specific magnitude; record statistics and extreme value theory are applied for extremely rare events (Rahmstorf and Coumou 2011; Coumou et al 2013; Lewis and Karoly 2013). For some recent heat waves, the fraction attributable risk is so high that it is found to be extremely unlikely that those 
events would have happened in the absence of climate change (Stott et al 2004; Lewis and Karoly 2013; Christidis et al 2014).

\subsection{Objective of thesis}

Assessing the role of anthropogenic forcing in observed impacts of climate change is thus a relevant and timely issue. Regardless of the role of anthropogenic forcing in the observed effects, assessments of resilience and vulnerability benefit from an advanced understanding of the interaction between climatic drivers and other important drivers of change.

Public interest is focussed on climate change impacts on human systems, an area that is particularly challenging for science due to the important roles of other drivers and the capacity for planned adaptation that apply here. Similarly, event attribution has attracted considerable attention in recent years, even though the attribution of loss and damage from extreme events to anthropogenic climate change is not currently feasible (James et al 2014).

This thesis aims to elucidate the extent to which the impacts of anthropogenic climate change can be detected worldwide, in particular within human and managed systems. It provides a clear analytical framework for the detection and attribution of observed impacts of climate change and focusses on two overarching questions: First, how has the assessment of the detection and attribution of observed climate change impacts evolved over the last two decades? Second, how do the observed impacts of recent climate trends relate to anthropogenic forcing? More specifically, each of the chapters will address at least one of the following five research questions:

1) What are clear and workable definitions and protocols for detection and attribution in the context of the observed impacts of climate change?

2) Can single extreme weather events and their consequent impacts be attributed to anthropogenic forcing?

3) What is the status of knowledge regarding observed climate change effects worldwide and how has the assessment and evidence base evolved over recent decades?

4) Can impacts of regional and local climate change be attributed to anthropogenic forcing and what are the dominant sources of uncertainty when establishing confidence in such findings?

5) What are the consequences of the challenges and limitations of detection and attribution studies for their use in informing risk assessments and international climate policy?

\subsection{Thesis outline}

This thesis is rooted in the work carried out during my position as a chapter scientist supporting the IPCC's working group two during the fifth assessment cycle. I had a leading role in the establishment, organization and ongoing work of a cross-chapter working group on detection and attribution within 
the WGII AR5 writing team, formed by authors delegated from all regional and sectoral chapters in addition to the detection and attribution chapter team. This thesis builds on the detection and attribution chapter' in the AR5 (Cramer et al 2014), the 'proof of concept paper' (Stone et al 2013) that outlined the approach taken by the chapter team, and a conference paper presenting the general challenges for a unified framework of detection and attribution (Hansen et al 2013).

This introduction, Chapter 1, defines the concepts that emerged from my work as a chapter scientist and provides the context for the further elaboration of those concepts in the following chapters.

Chapter 2 on linking local impacts to changes in climate - a guide to attribution summarizes the problem of detection and attribution of climate change impacts on natural and human systems and provides a protocol for the main steps of impact detection and attribution analysis based on multiple lines of evidence. The resulting framework allows an inclusive assessment of observed impacts in the context of human and managed systems, which is illustrated by its applications on several case studies, drawing from existing literature. Caveats and challenges of this process are highlighted in the discussion.

Chapter 3 on the attribution of an extreme event to climate change uses a simple statistical model of the occurrence of events to elucidate some fundamental issues in single-event attribution. It shows that single-event attribution is simply a re-casting of the attribution of a change in the overall rate of events. Confidence intervals are constructed based on a Poisson process model representing pre- and post-climate change situations. Results show that confident attribution of a single event generally requires a very large change in the overall rate.

In Chapter 4, the evolution of the evidence base for observed impacts of climate change on natural and human systems is documented across the three major systems (physical, biological and human) and eight world regions by comparing the results of IPCC'S third (TAR), fourth (AR4) and fifth (AR5) assessment reports. A matrix is developed to categorize the evidence base by quantity, spatial extent of evidence and number of impacted subcategories. The chapter discusses the influence of differing assumptions, regional monitoring capacity and data quality, and the magnitude of observed climate signals for the quality and extent of the available evidence.

Chapter 5 on attributing observed climate change impacts to human influence creates the previously 'missing link' between observed impacts of recent climate change and the cause of these regional changes in climate. To examine the role of anthropogenic forcing in observed effects of climate change, the spatial properties of the regional impact statements identified by Cramer et al (2014) are defined. Based on a new method for systematic climate attribution provided in the Annex, the confidence in attributing the observed climate trends in the corresponding areas to 
anthropogenic forcing is estimated. The results are analysed individually and compared to the impact attribution information. The patterns that govern the uncertainty of the assessment on both the climate and the impact side are subsequently discussed. Finally, both steps are combined in a multi-step assessment, confirming a discernible effect of anthropogenic forcing on natural and human systems.

Chapter 6 discusses some fundamental issues regarding the global distribution of climate change impacts and the challenges involved in conveying complex scientific information in a policy context. It highlights uneven global distribution of knowledge and stresses that the absence of evidence in some areas should not be interpreted as evidence for the absence of impacts.

Chapter 7 provides a synthesis of Chapters 2-6 and links back to the overall research questions introduced in Section 1.5. It elaborates on the consequences of the challenges and limitations of detection and attribution studies for their use in informing climate policy and critically evaluates the definitions and concepts introduced for impact attribution. 


\title{
2 Linking local impacts to changes in climate - a guide to attribution
}

\begin{abstract}
Assessing past impacts of observed climate change on natural, human and managed systems requires detailed knowledge about the effects of both climatic and other drivers of change, and their respective interaction. Resulting requirements with regard to system understanding and long-term observational data can be prohibitive for quantitative detection and attribution methods, especially in the case of human systems and in regions with poor monitoring records. To enable a structured examination of past impacts in such cases, we follow the logic of quantitative attribution assessments, however allowing for qualitative methods and different types of evidence. We demonstrate how multiple lines of evidence can be integrated in support of attribution exercises for human and managed systems. Results show that careful analysis can allow for attribution statements without explicit end-to-end modelling of the whole climate-impact system. However care must be taken not to overstate or generalize the results, and to avoid bias when the analysis is motivated by and limited to observations considered consistent with climate change impacts.
\end{abstract}

Publication corresponding to this chapter:

Hansen G, Stone D, Auffhammer M, Huggel C, Cramer W (2015) Linking local impacts to changes in climate - a guide to attribution. Regional Environmental Change doi: 10.1007/s10113-015-0760-y 


\subsection{Introduction}

Human interference with the climate system has been visible at global scales for some time, and is increasingly becoming apparent at regional scales (Stott et al 2010; Bindoff et al 2013). Consequently, the rigorous attribution of changes in local environmental conditions to changes in climate, and specifically the detection of climate change impacts in human systems and sectors interlinked with them, is gaining importance and public attention. Recent assessments of historical responses to climate change have drawn upon large amounts of direct observational evidence, applying formalized procedures for the detection and attribution of observed impacts (Rosenzweig \& Neofotis 2013; Cramer et al 2014).

While impacts of recent climate change are now documented for all continents and across the oceans, geographical imbalances and gaps in the documentation of impacts for human and managed systems remain. Based on scientific knowledge about the sensitivity of many human and managed systems to weather and climate variability, it is plausible to expect that recent climate change will have had a role in locally observed changes. However, confident detection of local effects in historical data remains challenging due to naturally occurring variability in both climate and potentially impacted systems, and the influence of other important drivers of change, such as land use, pollution, economic development and autonomous or planned adaptation (Nicholls et al 2009; Bouwer 2011; Hockey et al 2011). Often, the specification of a numerical model representing the entire climate-impact system may not be feasible. In those cases, the careful examination of the individual steps of the causal chain linking climate to impacts can still provide insight into the role of recent climate change for the system in question. The goal of this chapter is to provide guidance for such an approach to the detection and attribution of impacts of observed changes in climate.

Detection and attribution refer to the identification of responses to one or several drivers in historical observations, and a range of corresponding methods exists across research disciplines (Stone et al 2013). In the context of climate change research, detection and attribution methodologies have been developed mostly in the field of physical climate science, where a substantial literature presents various model-based statistical approaches to the question how effects of anthropogenic forcing can be identified in historical climate data (see Barnett et al 1999; Hegerl et al 2007; Bindoff et al 2013).

In contrast, efforts to develop overarching methods for the detection and attribution of observed impacts to climate change are limited (Stone et al 2009; Hegerl et al 2010; Stone et al 2013). Studies that explicitly attribute individual observed impacts of climate change to anthropogenic forcing of the climate system are rare. They usually combine observational data and process or statistical 
models of the impact system with climate model simulations representing the historic, anthropogenically forced state of the climate system and a hypothetical, natural state (Gillett 2004; Barnett et al 2008; Christidis et al 2010a; Marzeion et al 2014). In addition, methods have been developed to evaluate the role of anthropogenic forcing in large-scale patterns of multiple local impacts, mainly in ecology. These include the identification of so-called fingerprints of anthropogenic climate change in large sets of biological data (Parmesan and Yohe 2003; Root et al 2003; Poloczanska et al 2013), joint attribution (Root et al 2005) and joint attribution combined with spatial pattern congruence testing (Rosenzweig et al 2007; Rosenzweig et al 2008). Generally, these approaches aim at the identification of a generic impact of anthropogenic climate change which would emerge from analysing a large number of cases in parallel, given that it is often not possible to confidently attribute changes in individual local records to anthropogenic forcing for technical reasons (Rosenzweig and Neofotis 2013; Parmesan et al 2013).

The vast majority of impact studies are concerned with the identification of effects of regional changes in one or several climate variables in the context of multiple interacting drivers of change (Cramer et al 2014). Methods for detecting and explaining change are a key part of many disciplines studying natural, human and managed systems, and can be applied in the context of attribution to climate change. For example, reliable process-based models have been developed and applied in climate attribution analysis for some species and crops (Battisti et al 2005; Brisson et al 2010; Gregory and Marshall 2012). Statistical models are increasingly being used to assess large-scale effects of recent climate change (e.g. Lobell et al 2011b; Cheung et al 2013). However, explicit numerical modelling of the climate-impact system is not always feasible (see also Section 2.2). Instead, conclusions about cause and effect are often inferred from a combination of multiple lines of evidence, such as process understanding, local knowledge, field and model experiments, observations from similar systems in other locations or statistical analysis of observational data (see Section 2.3).

Below, we will focus on impact detection and attribution in a multi-step analysis, based on a structured examination of multiple lines of evidence. In doing so, we follow the approach proposed by Stone et al (2013) and applied in Cramer et al (Cramer et al 2014) and elsewhere in the WGII contribution to the Fifth Assessment Report (IPCC 2014a; IPCC 2014c). This approach is inspired by the framework laid out by the IPCC good practice guidelines for detection and attribution related to anthropogenic climate change (Hegerl et al 2010), but introduces the important modification that impact detection "addresses the question of whether a system is changing beyond a specified baseline that characterizes its behaviour in the absence of climate change" (IPCC 2014b). 
Detection of change in the climate system is concerned with the identification of a signal or trend beyond the short-term variability caused by internal processes. However, the underlying assumption of a stable natural baseline state, with stochastic-like variability superimposed may not be valid or practical in the case of some impact systems, particularly those involving humans. Many impact systems are undergoing constant change due to internal dynamics as well as external drivers which often interact and change over time. The observation of a trend in the overall behaviour of such a system, or a lack thereof, may not, on its own, be informative for assessing whether a response to climate change or any other driver has been detected (see also Section 2.2). The main concern of impact detection is to identify the effect of climate change against that of other drivers of change. Therefore, the detection of a climate change impact must involve the explicit testing for confounding factors. In that sense, impact detection cannot be entirely separated from attribution (see Stone et al 2013).

In this chapter we discuss the major steps involved in a complete evaluation of the causal chain from recent changes in climate to locally observed impacts. Following this introduction, we outline the required steps for a comprehensive impact detection and attribution analysis in Section 2.2. We focus on distinguishing the effects of climate change from those of non-climate drivers, rather than evaluating the anthropogenic contribution to the observed change in climate. In Section 2.3, we apply the resulting procedure in an analysis of several examples from human and managed systems, based on available literature. Those cases illustrate some of the major challenges involved, including the treatment of systems undergoing change from multiple drivers, and the integration of different types of evidence. We further discuss those challenges, and the limits and values of the detection and attribution of climate change impacts in Section 2.4, and provide brief conclusions in Section 2.5 .

\subsection{The five steps of an impact detection and attribution analysis}

The logic of quantitative detection and attribution analysis - if not the methods - can also be applied to qualitative studies and those that combine various sources of evidence. That logical flow follows from a classical hypothesis test. Briefly, to test whether climate change has had an effect on a system, a suitable regression or other model reflecting the knowledge of the system is specified. This model includes a possible effect due to climate change as well as other potentially influential factors. The statistical test is then based on comparing the goodness of fit of the model with climate change to that of the model without climate change. In both cases, the model is fitted by optimizing a measure of the goodness of fit. If the correctly specified model that includes the effect of a changing climate provides a significantly superior fit than the model that does not, we conclude that the data 
are not consistent with the null hypothesis that climate change has not had an effect: in other words, we have detected a climate change impact. If we are also interested in the magnitude of the contributions of the various drivers, the fitted model provides a way of assessing these (e.g. based on the regression parameters).

The focus on impacts of recent climate change mostly restricts attention to cases in which the design involves a trend in climate (which may, in turn, be consistent with the effect of anthropogenic forcing). The identification of a trend over time in relevant climate variables is therefore part of the analysis. It is important to note that in order to avoid bias, the hypothesis taken as the starting point should not be formulated from the same data used to test it. Rather, it may be drawn from theory, e.g. model predictions, or independent data, such as observations in a similar system in a different location. It can also be helpful to differentiate between known external drivers of a system, which are explicitly accounted for in the specification of the baseline behaviour, and confounding factors such as measurement errors, data bias, model uncertainty and influences from other potential drivers that are not explicitly considered in the study set-up (Hegerl et al 2010).

Below, we outline the major steps involved in a comprehensive detection and attribution analysis in the context of climate change impacts (see Figure 2.1).

1) Hypothesis formulation: Identification of a potential climate change impact;

2) Observation of a climate trend in the relevant spatial and temporal domain;

3) Identification of the baseline behaviour of the climate-sensitive system in the absence of climate change;

4) Demonstration that the observed change is consistent with the expected response to the climate trend and inconsistent with all plausible responses to non-climate drivers alone (impact detection);

5) Assessment of the magnitude of the climate change contribution to overall change, relative to contributions from other drivers (attribution). 


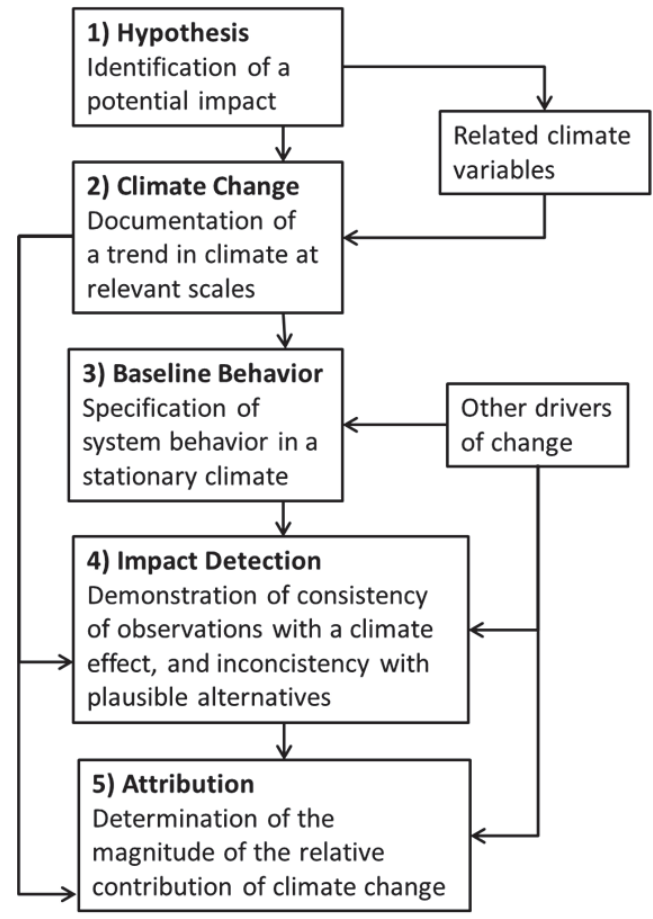

Figure 2.1: Schematic of the five steps of detection and attribution of observed climate change impacts. Note that in practice the specification of the baseline behaviour and the detection and attribution steps may be performed in parallel, given they all require explicit examination of all drivers of change in the system.

\subsubsection{Hypothesis}

A common source of hypothesis is a prediction of an effect of expected anthropogenic climate change based on system understanding. For example, if an impact of future anthropogenic climate change has been predicted in an earlier analysis, one could test whether that effect is now detectable in accumulated observations. Another source might be the detection of impacts in similar systems in other locations, or observations from the recent past, or from paleo records. Naturally, studies will also be motivated by observations of change in the climate-sensitive system; while it is unrealistic to ignore that motivation, efforts need to then be made to minimise the effect of the resulting selection bias or to evaluate its importance (Menzel et al 2006). A central part of this first step is the identification of metrics that characterise the expected response of the system to climate change. 


\subsubsection{Climate trend}

In order to detect an impact of observed climate change on a system, the climate must actually have changed and also have been observed to have changed for the relevant location and period. This condition distinguishes an impact study from a pure sensitivity analysis. Climate change is defined by the Intergovernmental Panel on Climate Change (IPCC) as "a change in the state of the climate that can be identified (e.g. by using statistical tests) by changes in the mean and/or the variability of its properties, and that persists for an extended period, typically decades or longer" (IPCC 2014b). In that sense, we consider a change in climate any long-term (e.g. 20 years and more) trend in a climate variable that is substantial in relation to short timescale variability, regardless of the cause of that trend.

A local climate trend is not necessarily caused by anthropogenic climate change. While it is plausible to assume that a local temperature trend that is consistent with the temperature trend in the larger area, which in turn has been attributed to global climate change, may also be caused by anthropogenic forcing, this must not be taken as proven. In general, individual and local climate records show higher variability than aggregated or global measures (Bindoff et al 2013). Local climate is influenced by topography and turbulence, but also by other local factors such as water management or land-use change. As a result, local trends may run contrary to or enhance the global warming signal, or may not emerge at all. Changes in atmospheric circulation patterns, or multidecadal natural variability could also generate local trends that differ from global ones. The question of how one might determine whether an observed trend is anthropogenically forced is beyond the scope of this chapter, but has been considered elsewhere (Stott et al 2010).

Systems may be sensitive to aspects of the climate other than the average, such as temperature exceeding $30^{\circ} \mathrm{C}$ during a certain period in plant development (e.g. Lobell et al 2011a). The chosen metric needs to reflect this aspect of the expected climate change.

\subsubsection{Baseline}

For some situations, the identification of a deviation from baseline behaviour is relatively straightforward: the metric shows a trend consistent in direction and magnitude with what one would expect under climate change, and that trend is also inconsistent with what could be plausibly expected as the effect of one or a combination of other known drivers in a stationary climate, either because those drivers are of insufficient magnitude or they mutually cancel. However, in most human and managed systems, we expect the observed overall response to be consistent with the combined effect of climate change and other drivers, but not with that of climate change alone. The 
failure to account for all drivers in the baseline may lead to erroneous conclusions about the influence of climate change on a system, as illustrated in Figure 2.2.

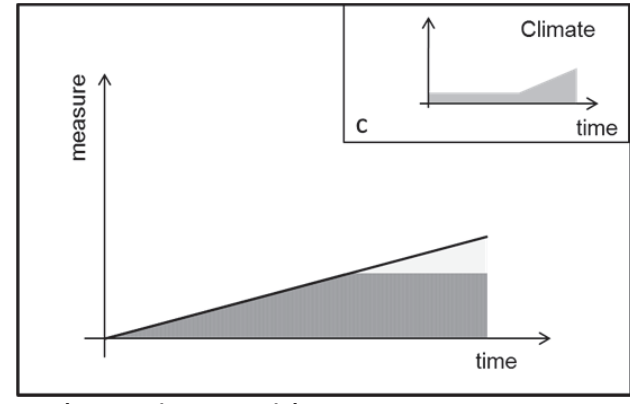

a. False negative: Potential type I error

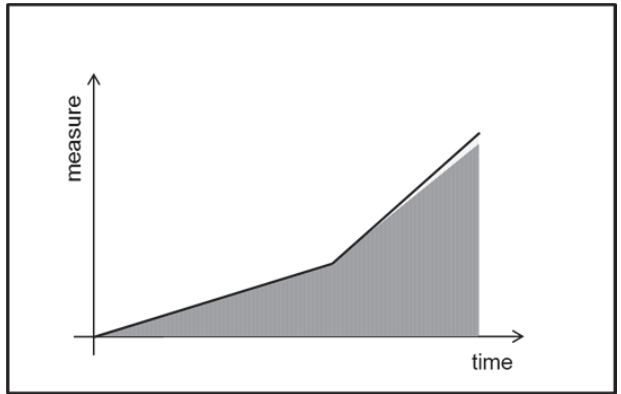

b. False positive: Potential type II error

Figure 2.2: Stylized examples of the time series of some measure representing a climate sensitive system which is responding in time to multiple drivers, one of them climate change (the corresponding time series of the climate variable for both cases is shown in Panel C, inserted in Panel A). The black line depicts the overall behaviour of the system, while the dark area represents the combined effect of non-climate drivers under stationary climatic conditions, and the light area represents the additional effect due to recent climate change. In Panel A, the baseline condition (dark area) shows a clear change midway through the record (e.g. due to a policy measure) but this is compensated by the influence of climate change. However, the resulting overall measure does not show a deviation from its historical pre-climate change trend, thus masking the existing climate change effect (potential type I error). In Panel B, the observed behaviour shows a change that is consistent in direction with a predicted climate change impact; however, the majority of that change happens due to a change in the baseline arising from other factors. This situation could lead to erroneous detection (potential type II error) or an overstatement of the climate effect.

So, in order to evaluate whether a climate change effect has been observed, the baseline behaviour of the system in the absence of climate change has to be specified (Stone et al 2013). For some systems, that behaviour may be non-stationary even in the absence of all external drivers.

As a world without climate change cannot be observed directly, the baseline must be constructed using statistical techniques, observations of analogous systems and/or system understanding expressed in the form of numerical or conceptual models. Specifying a reliable model is often hampered by lack of data, incomplete knowledge on processes and mechanisms involved in systems undergoing change from multiple stressors, limited understanding of causality within complex networks of social systems and how climate drivers and their perception influence those. In addition, research in qualitative social sciences focusses on descriptive, non-numerical understanding of how systems behave and interact and is often site or case specific. For a comprehensive assessment of impacts on humans systems, expectations of baseline behaviour may have to be developed and adopted based on qualitative methods. 


\subsubsection{Impact detection}

For natural, human and managed systems, impact detection addresses the question whether a system is changing beyond a specified baseline that characterizes behaviour in the absence of climate change (IPCC 2014b). In other words, impact detection requires the demonstration that an observed long-term change in a system cannot be fully accounted for by non-climate drivers. So, in order to detect an impact, it is not sufficient for climate change to be a plausible explanation, but it must also be shown that there is no (equally valid) alternative mechanism for the observed change (see also Figure 2.2).

In well-observed systems, a common way to investigate the effect of a driver on an outcome in the presence of other drivers is multiple regression analysis. To detect a climate change impact, the null hypothesis that climate change has not affected the outcome has to be tested, controlling for the impact of other drivers and confounding factors, including autonomous and planned adaptation. If the null hypothesis is rejected using a correctly specified model, a climate change impact has been detected. Following this statistical approach, a detection statement is always binary: an impact has (or has not) been detected at a chosen level of significance.

However, in many systems of interest, quantitative models representing causal relationships will be either impossible to construct or incompatible with the type of data available. In these situations not amenable to statistical testing, a detailed discussion of the role of other drivers and potential confounding factors such as measurement errors or data bias may provide a thorough evaluation of the various hypotheses. Though not directly comparable to the results of a rigorous analysis of longterm data, a clear and comprehensive qualitative analysis represents a valid form of evidence that should not be dismissed.

\subsubsection{Attribution}

Attribution needs to examine all drivers of change that influence the system, and evaluate their relative contribution to the detected change. Impact detection implies that climate change has had at least a minor role in the observed outcome. Assessing the magnitude of the contribution of climate change to an impact is a separate, but equally important matter in a detection and attribution exercise. An attribution statement needs a qualifier describing the relative importance of climate change to an observed impact. This involves either simply an ordinal statement (e.g. climate is the main influence responsible for a change) or a cardinal statement, which of course requires estimation of the exact relative magnitude of the contribution of climate change in relation to other drivers (see also Stone et al 2013). The descriptor relates to the size of the response to the climate driver relative to that to other drivers of change in the system, regardless of the direction of that 
change. While it may be relevant in other ways, the absolute size of the impact is not vital to the attribution statement.

A key challenge for all attribution exercises consists of accounting for non-additive effects of multiple drivers interacting on several temporal and spatial scales (see Parmesan et al 2013; Oliver and Morecroft 2014). While of particular concern for human and managed systems, such effects have also been shown in analyses of large datasets of biological changes (Darling and Cote 2008; Crain et al 2008).

\subsection{Impact attribution assessments - examples from human and managed systems}

In this section we provide examples which illustrate the challenges of thorough assessments of climate change impacts. The examples were chosen to cover a range of different conditions in terms of quality and type of evidence, and clarity of climate trends and observations. In line with the focus of this chapter, we selected examples from human and managed systems, and from world regions that are currently underrepresented in the literature. The assessments are based on available literature at the time of writing, and provide a summary of the more complex considerations detailed in the underlying literature. As detection is a necessary condition for attribution, the attribution step is omitted in cases where a climate impact has not been detected.

\subsubsection{Fisheries productivity on Lake Victoria}

\subsubsection{Hypothesis}

The inland fisheries of the Great Lakes are an important food source for the human population of Eastern and Southern Africa, with Lake Victoria having the largest freshwater lake fishery in the world. An expected outcome of anthropogenic climate change is warming of the Great Lakes, with faster warming at the surface increasing stratification (Lehman et al 1998; Verburg and Hecky 2009). Along with direct effects of the warming, the increased stratification is expected to limit nutrient recycling, consequently leading to increased abundance of algae and hypoxic conditions detrimental for the large fish which support the regional fishery industry (Lehman et al 1998). Hence, the fishery catch per unit effort would be expected to have decreased on Lake Victoria.

\subsubsection{Climate trends}

Atmospheric warming has occurred in the Great Lakes region (Verburg and Hecky 2009; NdebeleMurisa et al 2011), and lake surface waters appear to have warmed, too (Sitoki et al 2010; Loiselle et al 2014). Analyses of sediment cores suggest that the surface waters of other large Great Lakes have warmed to temperatures unprecedented in at least the last 500 years (Tierney et al 2010; Powers et 
al 2011). A strengthening of the thermocline (and hence increase in stratification) has been observed before 2000 , but appears to have weakened since, possibly due to variability in local wind regimes (Stager et al 2009, Sitoki et al 2010).

\subsubsection{Baseline}

The Great Lakes region has experienced a number of major environmental changes over the past few decades. The Nile Perch, a large predatory fish, and the Nile Tilapia were introduced in 19541964, and now comprise the bulk of the catch on Lake Victoria (Hecky et al 2010). A fundamental and rapid change in the fish community occurred in the early 1980s, and fishing effort has increased in recent decades (Kolding et al 2008). The invasive spread of the water hyacinth had disrupted lake access and transport on Lake Victoria in the 1990s until the more recent introduction of the weevil (Hecky et al 2010).

Much of the land surrounding Lake Victoria has been converted to agriculture, leading to increased run-off of nutrients (Stager et al 2009; Hecky et al 2010). Like warming, this would be expected to contribute to increased eutrophication, increased thermal stratification (by increasing algal abundance), and a shift in species composition and decreased species diversity.

\subsubsection{Impact detection}

The dramatic rise in both absolute fish catch and catch per unit effort observed on Lake Victoria during the 1980s coincided with the large-scale establishment of the introduced Nile perch. Altered predation dynamics due to a change in the light regime caused by the increased abundance of algae facilitated the success of the Nile perch (Kolding et al 2008; Hecky et al 2010). Another marked rise in catch of a native species in the 2000s is temporally linked to improved lake access after the establishment of efficient control of the water hyacinth (Hecky et al 2010). That rise is not reflected in other species and the relation to catch per unit effort is not documented; the Nile perch catch has been stable since the 1980s despite increased effort.

These catch changes are linked to other changes in the ecology of the lake which indicate the possible ultimate causes. Increases in primary productivity and algal abundance were documented in the decades before 2000, though both may have decreased since (Stager et al 2009; Hecky et al 2010; Sitoki et al 2010; Loiselle et al 2014). Increases are consistent with warming, increased nutrient supply from agricultural development and decreased abundance of planktivorous fish species caused by the introduced predators (Hecky et al 2010); the possible recent decrease in algal biomass could be indicative of a decreased catch per unit effort, as decreases in abundance of large predators allows populations of smaller fish species to recover. While the expected effects of species 
introductions can be distinguished from the expected response to warming, the responses to increased agricultural run-off and increasing fishing effort are harder to differentiate. Thus, while current evidence may suggest a response to warming beyond the responses to other drivers, considerable uncertainties remain.

\subsubsection{Attribution}

While anthropogenic climate change may become the dominant driver of the biology and productivity of the Great Lakes in future decades, current evidence is unable to distinguish whether the influence of warming has already been comparable to or much smaller than that of other drivers of environmental change in the region.

\subsubsection{Crop production in Southeast South America}

\subsubsection{Hypothesis}

In Southeast South America, significant increases in summer crop productivity and the expansion of agricultural areas have been observed over the last decades. Given that agricultural activity in the region is often constrained by the amount of rainfall, wetter conditions are expected to have contributed to these trends.

\subsubsection{Climate trends}

Southeast South America refers to the South American area south of $20^{\circ} \mathrm{S}$ and east of the Andes, excluding Patagonia, and includes the important agricultural production centre of the Argentinean Pampas, South-Eastern Brazil, Paraguay and Uruguay. Past precipitation and temperature trends are well-documented over the area (Giorgi 2002; Barros 2010; Magrin et al 2014). The region has warmed by roughly $1^{\circ} \mathrm{C}$ since the mid-1970s and the frequency of warm nights has increased. Over the same period, there has been a reduction in the number of overall dry days (Rivera et al 2013) and dry months in the warm season (Vargas et al 2010) and increases in precipitation, leading to a westward shift of the 600 and $800 \mathrm{~mm}$ isohyetal lines (Barros 2010; Doyle et al 2011).

\subsubsection{Baseline}

Across the region, socioeconomic factors such as policy incentives, market conditions, population growth and agronomic developments have positively affected cultivated area and agricultural productivity. The introduction of short-cycle soy varieties, no-till cropping systems and a general intensification of agriculture following macro-economic development contributed to the expansion 
of agricultural activities into formerly marginal land (Baldi and Paruelo 2008; Asseng et al 2012; Hoyos et al 2013).

\subsubsection{Impact detection}

Agricultural activity in the region is predominantly rain-fed. The wetter and partly warmer conditions observed since the 1970s are consistent with varying but substantial increases in yields observed in particular in those areas of Argentina, Uruguay and Southern Brazil where precipitation was the limiting factor in the first half of the century (Magrin et al 2005; Magrin et al 2007). In the semi-arid and sub-humid areas at the western and northern fringe of the Argentinean Pampas, increases in precipitation enabled a shift of the 'agricultural frontier' of about $100 \mathrm{~km}$ to the west into formerly semi-arid land (Barros, 2010).

In order to examine the role of different drivers in the expansion of agricultural land, Zak et al (2008) and Hoyos et al (2013) study the conversion of Chaco forest into crop and rangelands in an area at the Northern fringes of the Argentinean Pampas. They show that conversion rates in the Western part of their study region, which did not experience increases in precipitation, are considerably lower than those in the Eastern part, where they document upward trends in precipitation. As both regions exhibit otherwise very similar conditions, they conclude that climate change is an important enabling factor of the observed agricultural expansion, synergistic with technological changes and socioeconomic drivers. The case is less clear for the La Plata basin, where no such natural comparative area has been identified and studied, and the pattern of land types converted does not allow for a clear distinction of the role of the climate trends (Baldi and Paruelo 2008) as opposed to other factors.

Magrin et al (2005) use crop models to study the relative effects of observed changes in temperature and precipitation on yields in the Argentinean Pampas. They examine observed yields of four main crops (sunflower, wheat, maize and soy) in nine representative zones across the region. They conclude that climate change had non-negligible, favourable effects beyond that of technological changes. In a similar exercise for six zones that extended to locations in Uruguay and Brazil, Magrin et al (2007) found substantial positive climate change effects on yields in particular for summer crops. Effects were strongest in the originally drier regions.

\subsubsection{Attribution}

Recognizing what Zak et al (2008), call "synergistic consequences of climatic, socioeconomic, and technological factors", climate change is estimated to be a major driver of the observed increases in 
summer crop yields and of the expansion of agricultural land into the formerly semi-arid regions of South Eastern South America, while the magnitude of its role for other areas and crops is less clear.

\subsubsection{Agroforestry systems in the Sahel}

\subsubsection{Hypothesis}

Drought and heat-induced tree mortality is increasingly reported from many locations worldwide (Allen et al 2010). The pronounced drought over the Western Sahel for much of the second half of the $20^{\text {th }}$ century would be expected to result in negative impacts on agroforestry systems.

\subsubsection{Climate trends}

Rainfall decreased markedly over the western Sahel in the few decades after 1950, resulting in extremely dry conditions during the 1970s and 1980s. There has been some recovery of the rains since 1990, but totals remain well below the mid-20th century values (Greene et al 2009; Lebel and Ali 2009; Biasutti 2013). Like many regions of the world, the western Sahel has also warmed on the order of $1^{\circ} \mathrm{C}$ during that time (Niang et al 2014), promoting drought conditions.

\subsubsection{Baseline}

With a growing population, there has been a large increase in agricultural area in the western Sahel at the expense of wooded vegetation (Brink and Eva 2009; Ruelland et al 2011). The growing population may also be harvesting a larger amount of firewood. The basic structure of the agroforestry system and its management by local farmers have been reported to be fairly stable over the period covered here (Maranz 2009).

\subsubsection{Impact detection}

Over the past half century there has been a decrease in tree density in the western Sahel noted through field survey as well as aerial and satellite imagery (Vincke et al 2010; Ruelland et al 2011; Gonzalez et al 2012) and by local populations (Wezel and Lykke 2006). Because of their sensitivity to moisture deficits, trees would be expected to become less densely spaced during long-term soilmoisture drought. Tree mortality has been more pronounced for introduced or managed fruitbearing trees, which may be less adapted than the native vegetation to decadal-scale drought conditions, which appear typical of the western Sahel (Wezel and Lykke 2006; Maranz 2009).

The patterns of tree cover changes remain correlated with the combined effects of the warming and drying trends after accounting for the effects of other factors (Gonzalez et al 2012). Moreover, the enhanced mortality among introduced species in relation to indigenous species is more consistent 
with the effect of climate change than with that of the other drivers listed above (Wezel and Lykke 2006; Maranz 2009).

\subsubsection{Attribution}

The harvesting of firewood does not appear to have played a substantial role in the decrease in tree density (Gonzalez et al 2012). The shift from wooded to agricultural areas is substantial (Brink and Eva 2009; Ruelland et al 2011), and the decreases in tree density are correlated with proximity to human presence (Vincke et al 2010). However, both the warming and decreased rainfall trends appear to have played at least as large a role in the overall decrease in tree density (Gonzalez et al 2012), though this has not been examined specifically for fruit-bearing trees.

\subsubsection{Wildfire in Australia}

\subsubsection{Hypothesis}

Many high-impact fires occurred over the last decade, amongst them the 2009 'Black Saturday' bushfires, which were reported as one of the worst natural disasters in the history of Australia with 173 lives lost and around 2300 homes plus other structures destroyed (Crompton et al 2010). Bushfires occur naturally in Australia, and many of the influencing parameters are directly (temperature, precipitation and windiness) or indirectly (available fuel, land use and cover, fire history) susceptible to climate change (Williams et al 2009), with fire risk expected to increase under climate change (Reisinger et al 2014). Hence a possible increase in fire hazard due to recent climate change may have translated into increased damages from wildfire.

\subsubsection{Climate trends}

Increases in aggregate climate indices such as average temperature, maximum temperatures and the length of hot-spells have been detected on continental scale, albeit with strong seasonal and regional variations (Alexander and Arblaster 2009; Trewin and Vermont 2010). Composite indices such as the McArthur Forest Fire Danger Index (FFDI) have been developed to capture the combined influence of relevant meteorological variables such as temperature, relative humidity, wind speed and direction and antecedent precipitation for the assessment of fire risk. A trend in the FFDI toward increasing danger has been observed since 1970 over large parts of Australia, especially in the South and South East, with a clear signature of annual and decadal climate modes such as the El Niño Southern Oscillation and the positive phase of the Indian Ocean Dipole (Mills et al 2008; Clarke et al 2013). 


\subsubsection{Baseline}

Damages from wildfire have increased over the course of the century, consistent with the observed climate trends, but also with the effects of an increased number of exposed assets (such as settlements built in or close to fire prone bush land) and increases in population. Better fire management and improved forecasting may counteract these trends, however their influence has not been quantified (Crompton et al 2011; Nicholls 2011).

\subsubsection{Impact detection}

No detectable trend has been found in building damages or losses of life normalized against trends in population and number of dwellings over the last century or decades (Crompton and McAneney 2008; McAneney et al 2009; Crompton et al 2010). The normalization process does not account for all factors that influence vulnerability, for instance precautionary measures of individual home owners or collective measures of changing spatial planning in order to reduce risk. Several of these factors have been explored in the literature, often with a focus on specific regions or events. Examples include the role of the 'prepare, leave early or stay and defend' policy in New South Wales, or the reduction of community vulnerability through improved risk management (Haynes et al 2010; O'Neill and Handmer 2012; Whittaker et al 2013). Damage from extreme fires is mainly controlled by exposure, as structures built in close proximity to or within bush land are virtually impossible to defend during extreme fire conditions (Chen and McAneney 2004). In the Greater Melbourne area, encroachment of suburban dwellings into bush land has led to an increase in the number of exposed dwellings (Butt and Buxton 2009; Buxton et al 2011).

Crompton et al (2011), in a reply to Nicholls (2011), discuss and dismiss several factors (including improved fire management, forecasting and individual home owners' defence measures) that could be masking a trend consistent with a climate signal in the overall loss statistics. They conclude that an influence of anthropogenic climate change "is not ruled out by our analysis, but, if it does exist, it is clearly dwarfed by the magnitude of the societal change and the large year-to-year variation in impacts". In summary, an impact of climate change on observed damages from bushfires in Australia has not been detected.

\subsubsection{Urban coastal erosion and flooding in West Africa}

\subsubsection{Hypothesis}

Anthropogenic warming of the climate system is expected to cause widespread rises in sea level. West Africa has a number of low-lying urban areas particularly exposed to sea level rise, with 
increases in coastal erosion and flooding expected (Dossou and Glehouenou-Dossou 2007; Douglas et al 2008; Adelekan 2010).

\subsubsection{Climate trends}

There has been a lack of sustained tide gauge monitoring in West Africa over the past few decades (Church and White 2011; Fashae and Onafeso 2011). While satellite monitoring suggests rising total sea levels in the Gulf of Guinea, actual relative sea level changes at specific locations along the coast will depend on additional factors, such as human induced subsidence, or natural variations in ocean currents (Stammer et al 2013).

\subsubsection{Baseline}

The construction of ports has diverted coastal sediment transport around Cotonou, Benin, while marine sand quarries have already reduced the supply of sand to the city (Dossou and GlehouenouDossou 2007). Other plausible drivers of increased erosion have also been posited, these include subsidence due to oil exploration for Lagos, Nigeria and sediment trapping in reservoirs for most of the West African Coast (Ericson et al 2006; Douglas et al 2008).

\subsubsection{Impact detection}

Based on photographic evidence and comparison with satellite imagery, coastlines in some urban areas in the Gulf of Guinea seem to have been retreating over the past few decades (Dossou and Glehouenou-Dossou 2007; Fashae and Onafeso 2011). Ericson et al (2006) found that sediment trapping is the dominant cause of contemporary effective sea level rise for the Niger delta, with contributions from land subsidence due to oil exploration. Also, the construction of reservoirs on the Volta has led to a sharp decrease in sediments moving across the West African coast, passing cities such as Cotonou and Lagos. Given the lack of long-term monitoring of local sea level and coastal erosion, as well as the various possible drivers of coastal erosion, it is currently not possible to examine whether an anthropogenic climate change signal has been detected.

\subsection{Discussion}

This chapter was motivated by an apparent inconsistency between the accepted view that climate change is already impacting a number of vulnerable human and managed systems and the relative lack of documented evidence of observed impacts of climate change for those vulnerable systems. A large body of literature exists concerning the sensitivity of such systems to climate and to future climate change, but there is comparatively little documentation of observed impacts of climate change (Cramer et al 2014). 
A major factor explaining this gap consists in the lack of calibrated long-term monitoring across sensitive systems and regions, which would provide the observational basis that underpins detection and attribution analysis. Under the United Nations Framework Convention on Climate Change (UNFCCC), nations are obligated to monitor their respective contributions to anthropogenic forcing through standardized national greenhouse gas inventories, but no such inventory scheme or standard exists for impacts of climate change.

Detection and attribution studies are virtually impossible for impacts in some regions due to the absence of an observational basis. For example, to determine how sea level rise might be affecting urban coastal areas in West Africa (see 2.3.5) the current ambiguity over whether relative sea level has actually risen along the urban coastlines is a hindrance. Innovate methods exist to fill in such gaps, for instance through analysis of archival footage or consulting local and indigenous knowledge, and can provide valuable tools in some cases (Rosenzweig and Neofotis 2013).

The five examples discussed in Section 2.3 draw on disparate studies across disciplines for a comprehensive analysis of the role of observed climate change in the changes that various systems have experienced during recent decades. However, they also illustrate some of the challenges involved in the detection and attribution of impacts of climate change. For example, the ecosystem of Lake Victoria faced the introduction of large predatory species, and subsequently a regime shift occurred. Predicting the ecosystem response to such major unprecedented change would be challenging even if the underlying ecosystem dynamics were well-understood. While it is plausible to assume that increased precipitation will have contributed to increases in agricultural productivity in Southeast South America, it is very difficult to disentangle the influence of the climate trend from that of technological development and socioeconomic conditions for parts of the region. Similarly, complex factors related to exposure preclude the detection of a climate-related signal in damages from bushfire in Australia. In the case of West Africa, the monitoring of all drivers contributing to coastal erosion and flooding, as well as the documentation of the actual changes remains insufficient.

In some cases though, the examples also point to ways forward. Local knowledge has been valuable in assessing the role of rainfall decreases in the thinning of western Sahelian forests, similar to what has long been documented for Inuit observations of change in the Arctic (e.g. Nichols et al 2004; Krupnik and Ray 2007; Weatherhead et al 2010). Sediment cores provide proxy evidence that the current warming of the African Great Lakes is, essentially, unprecedented. Examination of historical aerial and satellite photography provided important insights about the baseline in several of the case studies. The roles of some potential drivers for Australian bushfire damage were elucidated by comparative analyses across fire events, regions and other dimensions. 
Several examples point to the synergistic effects of changes in climate and other drivers, for instance the enabling role of the precipitation increases for extension of agricultural activity (2.3.2), or the role of warming and weakening winds in triggering the ecosystem shift in Lake Victoria (2.3.1). To adequately capture the role of climate change in the light of other factors that may act as additional stressors, provide resilience or create synergistic effects different from the effect of any individual driver remains a central challenge for impact attribution.

A fundamental issue we have only touched upon briefly concerns the end point of attribution studies. For large parts of the community studying climate change and its impacts, as well as many stakeholders, 'attribution' is used as a synonym for 'attribution to anthropogenic forcing'. As one of the key motivations for detection and attribution research is to inform the UNFCCC, this end point has often been considered the main goal (Zwiers and Hegerl 2008). This is important in the context of potential litigation for adverse impacts of climate change (Grossman 2003), and may become relevant for the recently established "Warsaw International Mechanism for Loss and Damage" under the UNFCCC (James et al 2014). To assess the relative role of anthropogenic versus natural forcing in observations provides a means to estimate whether recent and current impacts might be expected to persist, and to calibrate predictions of future impacts made with other methods. However, as we have shown, it is often very difficult to detect climate change effects in observed records, and to disentangle the impacts of climate change from those of other drivers of change. Clearly, attribution of observed impacts to anthropogenic climate change adds another layer of complexity to an already challenging exercise.

Impact attribution research improves the understanding of vulnerabilities to long-term climatic trends, including interactions and non-additive effects of multiple drivers, for which identification of the underlying driver of the observed climate change may not be relevant (Parmesan et al 2011; Parmesan et al 2013). Impact detection and attribution provides important insights from 'real world' conditions as compared to experimental conditions or idealized models. Such knowledge is essential to identify the most adequate adaptation strategies and resilient pathways. Given the increasing rate of climate change and possible threshold behaviour in impacted systems, as well as ongoing adaptation and general development, caution must be applied when inferring conclusions about future climate change impacts from observations.

It is also essential to be clear about the difference between the estimation of sensitivity to weather, and the observation of an impact of climate change. This applies especially with regard to the perception of manifestations of climate variability, such as severe drought or storms. For many human and managed systems, impacts of extreme weather or climate shocks are the rare occasion where a clear climate-related signal can be detected. However, while the impact of a particular 
extreme can be an important indicator of sensitivity to climate, it does not by itself constitute a climate change impact (Allen et al 2007; Stott et al 2013; Hulme 2014).

\subsection{Conclusions}

Detection and attribution of climate change impacts provides the most complete and consistent analysis possible of the cause-effect chain, combining all possible sources of information in a coherent evaluation. While setting a high bar, the distinction between impacts that have been observed in data and linked to climate change with confidence and those that are predicted to occur but cannot be detected and attributed by science (as yet) has proven useful. However, caution must be applied both ways when interpreting results. The lack of documented impacts attributable to climate change should not be misread as evidence for the absence of such impacts. On the other hand, it is true that for many historic impacts on human systems, non-climate related drivers are equally or more important than recent climate change and must be accounted for.

There may be cases where data are insufficient to detect an impact, while given climate trends and known sensitivity strongly suggests that climate change will have affected the system. While we support the use of different types of evidence, and the application of interdisciplinary methods to establish causality, the fact remains that observational evidence demonstrating a long-term effect is needed for impact attribution. Or to put it another way - you cannot attribute something you have not detected.

Detection and attribution analysis can be a powerful tool in understanding how and why our world is changing, albeit its cost is the need to possess the necessary observations and understanding, which remains poor in many areas. To identify those gaps, to determine whether they can be filled, and if so to prioritize research to address them, will lead to a more comprehensive and inclusive understanding of the impacts of climate change. 


\section{On the attribution of a single event to climate change ${ }^{2}$}

\section{Abstract}

There is growing interest in assessing the role of climate change in observed extreme weather events. Recent work in this area has focussed on estimating a measure called attributable risk. A statistical formulation of this problem is described and used to construct a confidence interval for attributable risk. The resulting confidence is shown to be surprisingly wide even in the case where the event of interest is unprecedented in the historical record.

Publication corresponding to this chapter:

Hansen G, Auffhammer M, Solow AR (2014) On the Attribution of a Single Event to Climate Change. Journal of Climate 27:8297-8301.

\footnotetext{
${ }^{2}$ In this chapter, the term 'climate change' is used in the diction of the Framework Convention on Climate Change (UNFCCC) and refers to 'a change of climate which is attributed directly or indirectly to human activity that alters the composition of the global atmosphere and which is in addition to natural climate variability observed over comparable time periods'.
} 


\subsection{Introduction}

Climate change is predicted to increase the frequency of extreme weather events like intense hurricanes (Webster et al 2005) and heat waves (Meehl and Tebaldi 2004). It is natural, therefore, to ask when an event such as the European heat wave in 2003 or Hurricane Sandy in 2012 occurs if it can be attributed to climate change. This attribution question has gained some prominence with efforts to assess liability for weather-related damages due to climate change (Allen 2003). Recent work on single-event attribution has focussed either implicitly or explicitly on a quantity known as attributable risk (Stott et al 2004; Rahmstorf and Coumou 2011; Bindoff et al 2013). The purpose of this chapter is to present a statistical formulation for attributable risk and to discuss its estimation with a particular emphasis on the construction of a confidence interval.

\subsection{A statistical formulation}

A natural statistical formulation of single-event attribution is in terms of a stochastic point process (Cox and Isham 1980). A stochastic point process is the classical model of a series of events occurring in some way randomly through time. Such models have been used to describe a variety of extreme weather events including heat waves (e.g. Furrer et al 2010) and hurricanes (e.g. Jagger and Elsner 2006). We note that the definition of the events of interest can include features such as intensity, location and seasonality - for example, wintertime exceedances of a temperature threshold or Category 5 hurricanes above a certain latitude.

A point process is partially characterized by a rate function that gives the instantaneous frequency of events. When this rate function is constant, the point process is said to be stationary. For a stationary point process with constant rate $\mu$, the expected number of events in a period of length $T$ is $\mu T$. For simplicity, we will focus here on the case where climate change causes a shift from one stationary point process to another stationary point process. As discussed below, however, the results presented in this chapter also apply to the non-stationary case.

Single-event attribution asks: Given that an event has occurred after the climate has changed, was it or was it not caused by climate change? This question implies that, once climate has changed, the point process of events represents the superposition of a point process of events that would have occurred in the absence of climate change and a point process of events that would not have occurred in the absence of climate change and are, therefore, attributable to climate change. Moreover, these point processes must be independent - otherwise, the former would inherit a climate change effect through the latter. 
Suppose that the rate before climate change is $\mu$. Following climate change, this rate increases to $\beta \mu$ with $\beta \geq 1$. It is straightforward to show that, conditional on an event occurring after the climate has changed, the probability $p$ that it was caused by climate change is:

$$
p=1-1 / \beta
$$

It is this probability that most recent papers on single-event attribution seek to assess. Borrowing from epidemiology, the probability in (1) is referred to as the risk attributable to climate change or simply the attributable risk (Walter 1976). The definition of attributable risk only makes sense if $\beta \geq 1$ - that is, if climate change increases the rate of events. In cases where climate change decreases this rate, the quantity $1-\beta$ is the risk attributable to the absence of climate change for an event that occurred prior to climate change.

For convenience, we refer to a comparison of event rates before and after climate change. In practice, it is common to compare the rate in an earlier period to the rate in a later period without assuming that the former is completely free from the effect of climate change. In that case, the issue is one of attribution to a change in climate that has occurred between the two periods.

\subsection{Estimation of attributable risk}

In practical applications, attributable risk is not known and has to be estimated. In this section, we discuss this estimation with a particular focus on the construction of a confidence interval.

Let the random variable $X$ be the number of events in a pre-climate-change period of length $T_{1}$ and the random variable $Y$ be the number of events in a post-climate-change period of length $T_{2}$. The counts $X$ and $Y$ can be based either on historical records or on simulations from a climate model. In the former case, it is important that the event of interest not be selected because of its rarity in the pre-climate-change record. We will assume that both pre- and post-climate change events follow stationary Poisson processes. For a stationary Poisson process, the numbers of events in nonoverlapping periods are independent Poisson random variables with means proportional to the lengths of the periods (Cox and Isham 1980). As noted below, the Poisson model can be extended to allow for a non-stationary rate function. Although not all point processes are Poisson processes, there is theoretical support for their use in modelling rare events (Barbour 1988).

Let $x$ and $y$ be the observed values of $X$ and $Y$, respectively. Pryzyborowski and Wilenski (1940) gave an expression for the joint distribution of independent Poisson random variables. For the model outlined here, the probability of observing $x$ and $y$ can be decomposed as: 


$$
\begin{gathered}
\operatorname{prob}(X=x, Y=y)=\frac{\left(\mu\left(T_{1}+\beta T_{2}\right)\right)^{x+y} \exp \left(-\mu\left(T_{1}+\beta T_{2}\right)\right.}{(x+y) !} \times \\
\frac{(x+y) !}{x ! y !}(\tau /(\tau+\beta))^{x}(\beta /(\tau+\beta))^{y}
\end{gathered}
$$

where $\tau=T_{1} / T_{2}$. The first term is the Poisson probability of observing a total of $x+y$ events and the second term is the conditional probability that $x$ of these events occurred in the pre-climate-change period and $y$ occurred in the post-climate-change period. This latter probability is given by the binomial distribution with $x+y$ trials and success probability $\tau /(\tau+\beta)$. The maximum likelihood (ML) estimates of $\mu$ and $\beta$ are the natural ones:

$$
\begin{aligned}
& \hat{\mu}=x / T_{1} \\
& \hat{\beta}={ }^{\tau y} / x
\end{aligned}
$$

where $x$ and $y$ are the observed values of $X$ and $Y$ and the ML estimate of attributable risk $p$ is:

$$
\hat{p}=1-x / \tau y
$$

Because there is positive probability that $Y=0$, this estimate has neither finite mean nor variance. This can be avoided by conditioning on the event that $Y>0$ so that $Y$ has a so-called 0 -truncated Poisson distribution. This conditioning seems reasonable as at least one post-climate change event must have occurred to trigger the attribution exercise. Rather than pursue this here, we will instead focus on the construction of a confidence interval for $p$.

We will proceed as follows. If the lower and upper bounds of a $1-\alpha$ confidence interval for $\beta$ are $\beta_{L}$ and $\beta_{U}$, respectively, then the lower and upper bounds of a $1-\alpha$ confidence interval for $p$ are $1-1 / \beta_{L}$ and $1-1 / \beta_{U}$, respectively, so that a confidence interval for $p$ can be constructed from a confidence interval for $\beta$. Under the model outlined above, $\beta$ is the ratio of Poisson means. The literature on constructing a confidence interval for the ratio of Poisson means dates back at least to Chapman (1952) and several approaches are described in Price and Bonett (2000). Here, we will adopt the common approach of eliminating the nuisance parameter $\mu$ by conditioning on the observed value $n=x+y$ of $X+Y$. As noted, the conditional distribution of $X$ and $Y$ given that their sum is $x+y$ is binomial with $x+y$ trials and success probability $\tau /(\tau+\beta)$. If $L$ and $U$ are the lower 
and upper bounds respectively of a $1-\alpha$ confidence interval for this probability, then the corresponding lower and upper bounds of a $1-\alpha$ confidence interval for $\beta$ are:

$$
\beta_{L}={ }^{\tau(1-U)} / U
$$

and

$$
\beta_{U}=\tau(1-L) / L
$$

respectively. In this step, we will use the approximate confidence interval originally proposed by Wilson (1927) and recommended by Brown et al (2001)) for a binomial probability with:

$$
L=\frac{x+z^{2} / 2}{n+z^{2}}-\frac{z \sqrt{n}}{n+z^{2}} \sqrt{\hat{q}(1-\hat{q})+z^{2} /(4 n)}
$$

and

$$
U=\frac{x+z^{2} / 2}{n+z^{2}}+\frac{z \sqrt{n}}{n+z^{2}} \sqrt{\hat{q}(1-\hat{q})+z^{2} /(4 n)}
$$

where $z$ is the upper $\alpha / 2$-quantile of the standard normal distribution and $\hat{q}=x / n$. Finally, the corresponding lower and upper bounds of an approximate $1-\alpha$ confidence interval for $p$ are:

$$
p_{L}=1-1 / \beta_{L}
$$

and

$$
p_{U}=1-1 / \beta_{U}
$$

respectively.

The actual coverage of the Wilson confidence interval is close to its nominal level unless $x$ is close to 0 . For $x$ small but positive, Brown et al (2001) described a modification that improves coverage. 
Although we will not consider this here, we will consider the important case where $x=0$ (i.e. the events of interest are without precedent prior to climate change). In this case, the upper bound of an exact $1-\alpha$ confidence interval for $\tau /(\tau+\beta)$ is $1-\alpha^{1 / n}$ (Jovanovic and Levy 1997). It follows that the lower bound of an exact $1-\alpha$ confidence interval for $p$ when $x=0$ is:

$$
p_{L}^{0}=1-\left(1-\alpha^{1 / n}\right) /\left(\tau \alpha^{1 / n}\right)
$$

As noted, although we have focussed on the case where both the pre- and post-climate change Poisson processes are stationary, the results of this section extend to the case where either or both is non-stationary. Briefly, for a non-stationary Poisson process with time-varying rate function $\mu(t)$, the number of events in the interval $(u, v)$ has a Poisson distribution with mean $\bar{\mu}(v-u)$ where:

$$
\bar{\mu}=\int_{u}^{v} \mu(t) d t /(v-u)
$$

is the mean rate during this interval. It follows that $\beta_{L}$ and $\beta_{U}$ in (6) and (7) are the bounds of a $1-\alpha$ confidence interval for the ratio of the mean rate in the post-climate-change period to the mean rate in the pre-climate-change period and consequently that $p_{L}$ and $p_{U}$ in (10) and (11) are the bounds of a $1-\alpha$ confidence interval for attributable risk based on these mean rates. Of course, if the rate function increases continuously during the post-climate change period, the attributable risk for events late in this period is greater than that for events earlier in the period. It is possible to develop a continuous measure of attributable risk, by modelling the rate function but the construction of a confidence interval would be more challenging.

\subsection{Results}

To illustrate the calculations outlined in the previous section, Table 3.1 presents the ML estimate $\hat{p}$ and the bounds of the approximate 0.95 confidence interval for $p$ for selected positive values of $x$ and $y$ and selected values of $\tau$. A negative lower confidence bound in Table 3.1 indicates that the confidence interval for $\beta$ contains values less than 1 (i.e. a decrease in the rate of events cannot be ruled out). Table 3.2 presents the lower bound of a 0.95 confidence interval for $p$ for selected values of $y$ with $x=0$ and selected values of $\tau$. In all cases in Table 3.2, the point estimate of $p$ is equal to 1 as is the upper bound of the confidence interval. Again, a negative lower confidence bound indicates that a decrease in the rate of events cannot be ruled out. It is clear that even establishing that the 
rate of events has increased with climate change (i.e. $p>0$ ) may not be possible when the events are rare. This is true even if the events are without precedent in the pre-climate-change record. Even if this basic fact can be established, a surprisingly large number of events may be needed before attributable risk can be estimated with high confidence.

Table 3.1: Maximum likelihood estimate $\hat{\boldsymbol{p}}$ of attributable risk and lower $\boldsymbol{p}_{L}$ and upper $\boldsymbol{p}_{U}$ bounds of an approximate $\mathbf{0 . 9 5}$ confidence interval for $\boldsymbol{p}$ for selected values of $\tau, x$, and $y$.

\begin{tabular}{|r|r|r|r|r|r|}
\hline $\boldsymbol{\tau}$ & $\mathbf{x}$ & $\mathbf{y}$ & $\hat{p}$ & $\mathbf{p}_{\mathbf{L}}$ & $\mathbf{p}_{\mathbf{u}}$ \\
\hline $\mathbf{0 . 5}$ & 1 & 4 & 0.5 & -2.33 & 0.93 \\
\hline & 10 & 40 & & 0.01 & 0.75 \\
\hline & 25 & 100 & & 0.23 & 0.68 \\
\hline & 1 & 10 & 0.8 & -0.21 & 0.97 \\
\hline & 5 & 50 & & 0.51 & 0.92 \\
\hline & 25 & 250 & & 0.70 & 0.87 \\
\hline $\mathbf{1 . 0}$ & 2 & 4 & 0.5 & -1.33 & 0.89 \\
\hline & 20 & 40 & & 0.15 & 0.71 \\
\hline & 50 & 100 & & 0.30 & 0.64 \\
\hline & 2 & 10 & 0.8 & 0.19 & 0.95 \\
\hline & 10 & 50 & & 0.61 & 0.90 \\
\hline & 50 & 250 & & 0.73 & 0.85 \\
\hline $\mathbf{2 . 0}$ & 4 & 4 & 0.5 & -0.82 & 0.86 \\
\hline & 40 & 40 & & 0.23 & 0.68 \\
\hline & 100 & 100 & & 0.34 & 0.62 \\
\hline & 4 & 10 & 0.8 & 0.40 & 0.93 \\
\hline & 20 & 50 & & 0.67 & 0.88 \\
\hline & 100 & 250 & & 0.75 & 0.84 \\
\hline
\end{tabular}

Table 3.2: Lower bound $p_{L}^{0}$ of a 0.95 confidence interval for attributable risk for selected values of $\tau$ and $y$ with $x=0$.

\begin{tabular}{|r|r|r|}
\hline $\boldsymbol{\tau}$ & $\boldsymbol{y}$ & $\mathbf{p}_{\mathrm{L}}$ \\
\hline $\mathbf{0 . 5}$ & 1 & -37.0 \\
\hline & 2 & -5.94 \\
\hline & 5 & -0.64 \\
\hline & 10 & 0.30 \\
\hline $\mathbf{1 . 0}$ & 1 & -18.0 \\
\hline & 2 & -2.47 \\
\hline & 5 & 0.18 \\
\hline & 10 & 0.65 \\
\hline $\mathbf{2 . 0}$ & 1 & -8.5 \\
\hline & 2 & -0.74 \\
\hline & 5 & 0.59 \\
\hline & 10 & 0.83 \\
\hline
\end{tabular}

As a further illustration, we applied the methods of the previous section to data documenting intense (Categories 4 and 5) hurricanes in the North Atlantic over the period 1950-2012. These data were extracted from the Atlantic hurricane Best Track data set maintained at the United States National Hurricane Center (NOAA 2014). The effect of climate change on the frequency of such hurricanes and the quality of the historical data remain unsettled (Knutson et al 2010) and we stress that this is intended as an illustration. Over the 30-year period 1950-1979 there were a total of 39 
intense North Atlantic hurricanes while over the following 33-year period 1980-2012, there were 53 such hurricanes. If we assume that the effect of climate change over the entire 63-year period was to increase the rate of these hurricanes, then the ML estimate of the estimated probability that a hurricane in the later period is attributable to climate change is 0.19 and an approximate 0.95 confidence interval for this probability is $(-0.17,0.44)$. The negative lower bound of this confidence interval indicates that a decline in the rate of intense hurricanes between these periods cannot be ruled out. At the same time, the upper bound of 0.44 indicates that neither can a near doubling of this rate be ruled out. It is worth noting that the rate of intense hurricanes varies over both the seasonal timescale and the inter-annual timescale (e.g. due to ENSO variability), so this is an example of an application to a non-stationary process.

\subsection{Discussion}

This chapter has outlined a statistical formulation of the attribution of a single event to climate change and has used this formulation to provide a confidence interval for attributable risk. Formulating single-event attribution in this way raises two fundamental issues. First, as noted, underlying the concept of attributable risk is a dichotomy between events that would have occurred in the absence of climate change and events that would not. This dichotomy makes sense in epidemiology (and in other contexts). For example, some cases of lung cancer are caused by smoking, others are not, and it is natural to ask about the risk of lung cancer attributable to smoking. It is not so clear, however, that attributable risk makes sense in the context of climate change. While the effects of smoking are confined to the smoker (and perhaps those around him), the effect of climate change is pervasive and the notion that, once the climate has changed, some weather events would have occurred exactly as they did in its absence may not be tenable. To be clear, this is not at all to say that a change in the rate of events cannot be attributed to climate change, only that the superposition argument on which attributable risk is based may not be tenable.

Second, even if the notion of attributable risk makes sense in the context of climate change, the quantity $p$ is simply a function of the rates of events before and after climate change and not particularized to an individual event. To put it another way, the attributable risk is the same for all post-climate change events. In this sense, it is not really single-event attribution. Turning to the results of the previous section, it is clear that uncertainty about attributable risk can remain high unless both the number of observed events and the effect of climate change are large. 


\title{
4 The evolution of the evidence base for observed impacts of climate change
}

\begin{abstract}
Natural and human systems are increasingly affected by climate change. A synopsis of the documentation of scientific evidence for the observed effects of climate change in the third, fourth and fifth assessment reports of the Intergovernmental Panel on Climate Change shows that the amount of evidence available, the range of impacts observed and their geographical scope has expanded rapidly. Fifteen years ago, robust evidence for observed climate change impacts was almost exclusively available for the cryosphere and terrestrial ecosystems in mid to high northern latitudes or mountain regions. In contrast, the effects of climate change are now documented for all land areas and oceans, for both natural and human systems. Over the last decade, evidence has increased especially for impacts on marine ecosystems, food production and wildfire regimes. No recent progress has been found in the documentation of impacts of climate change related to sea level rise. Though the evidence base has improved substantially for regions in the Southern hemisphere and developing countries, the global distribution of observed impacts remains uneven.
\end{abstract}

\section{Highlights}

- The evidence base for observed climate change impacts has expanded rapidly since the third IPCC assessment report

- Analytic approaches vary between IPCC assessment cycles, but the pattern of impacts is robust

- Impacts are now observed for all land areas and oceans, and across natural and human systems

- Extensive impacts of climate change are still best documented for regions at mid to high northern latitudes

- Despite challenges in attribution, impacts on the food system and human health have clearly materialized

Publication corresponding to this chapter:

Hansen G (2015) The evolution of the evidence base for observed impacts of climate change. Current Opinion in Environmental Sustainability (accepted) 


\subsection{Introduction}

The signal of anthropogenic climate change is increasingly visible in manifestations of regional climate (Stott et al 2010; Bindoff et al 2013). Scientific studies of the impacts of climate change largely focus on expected effects of future changes in climate and the sensitivity of sectors and systems to such changes. Over the past two decades, research addressing observed impacts of recent climate change has increased substantially. While many changes are observed in the natural environment and in human systems, to establish a clear link to climate change is often difficult due to the influence of a multitude of other drivers. Scientists have developed and applied methods to determine the role of climate change in observed impacts under the label detection and attribution (Hegerl et al 2010; Stone et al 2013; see Chapter 2). A central requirement of such methods is the availability of long series of observational data, on both the climate and the impact side, and the explicit examination of all plausible drivers of change beyond mere correlation.

The most recent report by the Intergovernmental Panel on Climate Change (IPCC) concludes that "impacts of recent changes in climate on natural and human systems occur on all continents and across the oceans. This conclusion is strengthened by both new and longer term observations and through more extensive analyses of existing data" (Cramer et al 2014). This chapter examines the evolution of the evidence base documenting observed climate change impacts and its spatial distribution, based on a synopsis of the working group II contributions to IPCC's third (TAR), fourth (AR4) and fifth (AR5) assessment reports, which serve as a synthesis of the available literature ${ }^{3}$. It summarizes the status of knowledge on observed climate change impacts, highlights the most robust impacts and provides a review for some areas of new and emerging evidence.

Section 4.2 presents a graphical overview of the evolution and status of knowledge across three overarching systems (physical, biological and human and managed systems) and nine impact categories for IPCC world regions (Figure 4.1). The current status of knowledge is then more closely examined in Section 4.3. Extensive impacts observed across regions and globally are summarized and knowledge gaps identified and discussed. Central new findings of the AR5 are reviewed in more detail and extended to include relevant studies published after the AR5 was completed. In Section 4.4, a more comprehensive review of some recent developments regarding observed impacts of climate change on human and managed systems is singled out, followed by a conclusion and outlook

\footnotetext{
${ }^{3}$ Documentation of observed effects and definition of regions differ between the three assessment cycles. Central sources of this analysis were the chapters dedicated to observed impacts in IPCC AR5 (Cramer et al 2014) and AR4 (Rosenzweig et al 2007), and the ecosystems chapter of TAR (Gitay et al 2001); the latter complemented with information from other WGI (Folland et al 2001) and WGIl chapters (Arnell et al 2001) of the third assessment cycle. Detailed information on the approach for the evaluation, and criteria applied during the analysis can be found in the supplementary material to the corresponding publication.
} 
in Section 4.5. Throughout the chapter, the focus is kept on impacts of all long-term trends in climate, regardless of the role of anthropogenic forcing for that trend ${ }^{4}$.

\subsection{The evolution of the evidence base for observed impacts of climate change}

In this section, the evolution of the evidence base documenting observed impacts of climate change is summarized, based on the relevant chapters from the third, fourth and fifth IPCC assessment reports.

Approaches to the detection and attribution of impacts of climate change, as well as the definition of the terms used, have undergone gradual change over the course of time and differ between IPCC reports (Stone et al 2013). For example, while the AR4 provides a meta-assessment in order to link evidence of local impacts to anthropogenic climate trends, both the TAR and the AR5 focus on the impacts of regional climate trends ${ }^{1}$ and do not emphasize the role of anthropogenic forcing for those trends. In IPCC's AR5, the concept of 'impact detection' was introduced, extending the detection and attribution concept as presented in the IPCC good practice guidance paper on detection and attribution (Hegerl et al 2010). The TAR and the AR4 use hard criteria, such as length of data series and statistical significance of correlations, to define the scope of their analysis. In contrast, the AR5 assessment is based on expert judgement, integrating different sources and qualities of evidence, which usually include, but are not limited to, studies presenting statistical analyses of long-term sets of observational data. This approach contributes to the increased documentation of climate change effects for human systems as it facilitates the integration of qualitative evidence and local knowledge. The recent AR5 report also examines the interaction of climate change with other drivers and assesses the magnitude of the role of climate change compared to other drivers of change.

Available evidence is evaluated for nine impact categories, displayed in Table 4.1. These nine categories are identical to those used in AR5 and are aligned with the areas of evidence represented in the literature. To provide a consistent framework for the evaluation, minor adjustments to the original IPCC assessments were necessary. More information on the framework and underlying assumptions and adjustments can be found in the supplementary information provided for the corresponding publication.

\footnotetext{
${ }^{4}$ The most appropriate end point for impact attribution studies continues to be subject to debate (Parmesan et al 2013). The IPCC defines climate change as "a change in the state of the climate that can be identified [...] by changes in the mean and/or the variability of its properties, and that persists for an extended period, typically decades or longer", whereas its primary client - the United Nations Framework Convention on Climate Change - refers to "a change of climate which is attributed directly or indirectly to human activity that alters the composition of the global atmosphere" (IPCC 2014b), i.e. anthropogenic climate change.
} 
Table 4.1: Impact categories and associated subcategories used in this analysis. For human systems in particular, subcategories are not comprehensive but mirror the areas where evidence was reported.

\begin{tabular}{|c|c|c|}
\hline & Impact category & Subcategories \\
\hline \multirow{3}{*}{$\frac{\substack{\frac{\pi}{N} \\
\frac{1}{\alpha}}}{2}$} & Cryosphere & $\begin{array}{l}\text { Glacier volume/length; lake- and river ice; sea ice; lowland } \\
\text { and mountain permafrost; snow cover }\end{array}$ \\
\hline & Hydrology & $\begin{array}{l}\text { Changes in pattern and magnitude of river run-off and river } \\
\text { flow; lake size and warming/stratification; soil moisture } \\
\text { drought; floods; groundwater change }\end{array}$ \\
\hline & Coastal processes & $\begin{array}{l}\text { Coastal erosion; shoreline degradation; coastal flooding; } \\
\text { groundwater salinization; coastal permafrost degradation; } \\
\text { wetlands and coastal ecosystem changes due to sea level rise }\end{array}$ \\
\hline \multirow{3}{*}{$\begin{array}{l}\frac{.0}{00} \\
\frac{0}{0} \\
\frac{0}{0}\end{array}$} & Terrestrial ecosystems & $\begin{array}{l}\text { For terrestrial and freshwater systems: changes in species } \\
\text { abundance, composition, range or distribution, phenology and } \\
\text { productivity; synchronisation and ecosystem effects; } \\
\text { extinctions }\end{array}$ \\
\hline & Marine ecosystems & $\begin{array}{l}\text { For marine ecosystems, including sea birds and sea ice based } \\
\text { mammals, and temperature effects on coastal ecosystems: } \\
\text { changes in species abundance, composition, range or } \\
\text { distribution, phenology and productivity; synchronisation and } \\
\text { ecosystem effects; extinctions; invasive species }\end{array}$ \\
\hline & Wildfire & $\begin{array}{l}\text { Wildfire frequency; area burnt; intensity and duration of fires; } \\
\text { occurrence of mega fires; unprecedented wildfires }\end{array}$ \\
\hline \multirow{3}{*}{ 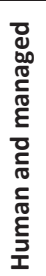 } & Food systems & $\begin{array}{l}\text { Cropping systems; agroforestry; livestock and pastoral } \\
\text { systems; inland and marine fisheries and aquaculture; } \\
\text { horticulture, viticulture and orchards }\end{array}$ \\
\hline & Human health & $\begin{array}{l}\text { Heat and cold-related mortality; health effects of extreme } \\
\text { heat; vector borne diseases; water borne diseases }\end{array}$ \\
\hline & $\begin{array}{l}\text { Livelihoods and other } \\
\text { socioeconomic indicators }\end{array}$ & $\begin{array}{l}\text { Livelihoods of rural populations, farmers, fishers and hunters; } \\
\text { adaptive responses; traditional and cultural practices and } \\
\text { values; infrastructure and travel }\end{array}$ \\
\hline
\end{tabular}

Each impact category is rated on a scale ranging from 'no' to 'extensive evidence' (see Table 4.2) based on a matrix combining indicators for three dimensions of evaluation: spatial coverage, range and variety of documented impacts, and the literature base supporting observed impacts. Each IPCC report is first assessed individually, followed by an aggregation step that includes the findings of the earlier report(s) into a list representing 'cumulative' impacts.

Table 4.2: Categories used in the evaluation of the scientific evidence base for observed impacts of climate change. More detailed information regarding the categories and the evaluation process can be found in the supplementary material to the publication corresponding to this chapter.

\begin{tabular}{|c|c|}
\hline & General description \\
\hline Zero & $\begin{array}{l}\text { No evidence or available evidence is anecdotal or does not clearly document } \\
\text { observed changes in both climate and impact over a sufficient period of time }\end{array}$ \\
\hline Emerging & $\begin{array}{l}\text { Individual but robust attribution finding with limited scope, or several more } \\
\text { uncertain or conflicting findings }\end{array}$ \\
\hline Moderate & More than one robust finding, but still rather narrow in terms of scope \\
\hline Solid & $\begin{array}{l}\text { Medium to large set of studies with broader scope both spatially and with regard to } \\
\text { impact traits covered }\end{array}$ \\
\hline Extensive & Large set of studies, continental scale, multi-trait across subsystems \\
\hline
\end{tabular}


During integration, the evaluation considers whether studies present evidence for new impact categories or are updating/confirming earlier findings. As each IPCC report builds on the findings of the preceding volume, the lists of cumulative impacts for AR4 and AR5 differ from the lists derived from AR4 or AR5 alone. Impacts that have been reported in an earlier report, are usually not highlighted in detail in the following volume, unless there have been substantial new or conflicting findings. During evaluation, two incidents were identified where later volumes reported less robust evidence for the same observation: For example, in the case of Sahel drought, the observed climate trend shifted towards slightly wetter conditions during the past decades. In the case of extinction of amphibians in Latin America reported in the AR4, additional research resulted in a lower confidence in the attribution of their extinction to climate change.

Figure 4.1 summarizes the results of the evaluation of available evidence for nine impact categories. Both the regional and sectoral scope of observed impacts has evidently expanded vastly since the TAR. More pronounced climate signals in many regions and the availability of longer data series enhance statistical confidence in attributing observed impacts to climate change and therefore contribute to the expansion of regional and sectoral coverage. Science still provides the most extensive evidence for climate change impacts on the cryosphere and terrestrial ecosystems. However, evidence increases substantially between AR4 and AR5 regarding effects on marine ecosystems. Evidence for impacts on coastal systems remains comparably weak and many of the documented impacts on coastal systems are related to thermokarst and permafrost thaw, as well as changes in sea ice regimes in the high northern latitudes, rather than to rising sea levels due to climate change. Similarly, temperature-mediated changes in snow regimes and melting glaciers are the main drivers of attributed hydrological impacts, as opposed to changes in precipitation.

The AR4 reports moderate evidence for impacts on human systems for some regions (Figure 4.1), which is substantiated - in particular for the food system - and extended to more impact categories in AR5. This is remarkable because the attribution of observed effects in human and managed systems is extremely difficult due to the presence of multiple confounders, including autonomous and planned adaptation. 


\begin{tabular}{|c|c|c|c|c|c|}
\hline \multirow{2}{*}{$\begin{array}{l}\text { Physical } \\
\text { systems }\end{array}$} & \multicolumn{3}{|c|}{ TAR } & AR4 & AR5 \\
\hline & CRY & HYD & COA & CRY HYD COA & CRY HYD COA \\
\hline Africa & P & $\bullet$ & & 0 & 0 \\
\hline Asia & & ? & 8 & 8 & \\
\hline Australasia & & - & & 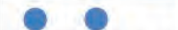 & \\
\hline Europe & & 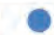 & - & 0 & 9 \\
\hline South America & & 0 & & P & \\
\hline North America & & - & ( & & \\
\hline Antarctic & - & & & - & O \\
\hline Arctic & & 0 & 0 & 0 & \\
\hline Small Islands & & & - & $\bullet$ & - \\
\hline
\end{tabular}

\begin{tabular}{|c|c|c|c|c|c|}
\hline \multirow{2}{*}{$\begin{array}{l}\text { Biological } \\
\text { systems }\end{array}$} & \multicolumn{2}{|r|}{ TAR } & \multicolumn{2}{|r|}{ AR4 } & AR5 \\
\hline & MES & TES WF & MES & TES WF & MES TES WF \\
\hline Africa & & & & O & C \\
\hline Asia & & & & 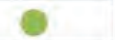 & 8 \\
\hline Australasia & - & $\bullet$ & (1) & 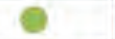 & \\
\hline Europe & 8 & & & • & 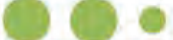 \\
\hline South America & & $=$ & & 0 & 8 \\
\hline North America & $\theta$ & & & 2 & \\
\hline Antarctic & P & $\bullet$ & & 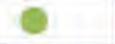 & \\
\hline Arctic & O & P & & - & (6) \\
\hline Small Islands & & & Q & & P \\
\hline
\end{tabular}

\begin{tabular}{|c|c|c|c|c|c|c|c|c|c|}
\hline \multirow{2}{*}{$\begin{array}{l}\text { Human } \\
\text { systems }\end{array}$} & \multicolumn{3}{|c|}{ TAR } & \multicolumn{3}{|c|}{ AR4 } & \multicolumn{3}{|c|}{ AR5 } \\
\hline & FS & $\mathrm{HH}$ & SE\& & FS & $\mathrm{HH}$ & SE\& & FS & $\mathrm{HH}$ & SE\& \\
\hline Africa & & & & 8 & & & & $\bullet$ & $\bullet$ \\
\hline Asia & & & & $\bullet$ & & & & $\bullet$ & $\bullet$ \\
\hline \multicolumn{10}{|l|}{ Australasia } \\
\hline Europe & & & & 0 & & & & & $\bullet$ \\
\hline South America & & & & & & & & & $\bullet$ \\
\hline \multicolumn{10}{|l|}{ North America } \\
\hline \multicolumn{10}{|l|}{ Antarctica } \\
\hline Arctic & & & $\bullet$ & & & C & & & \\
\hline Small Islands & & & & & & & & & $\bullet$ \\
\hline
\end{tabular}

\begin{tabular}{|l|c|}
\hline Legend & \\
\hline Zero & \\
\hline Emerging & $\bullet$ \\
\hline Moderate & \\
\hline Solid & \\
\hline Extensive & \\
\hline
\end{tabular}

Figure 4.1: Development of evidence base representing observed impacts attributed to recent climate change for physical systems [top panel: cryosphere (CRY), hydrology (HYD) and coastal processes (COA)], biological systems [centre panel: marine (MES) and terrestrial (TES) ecosystems, and impacts of wildfire (WF)], and human and managed systems [bottom panel: food systems (FS), human health (HH), and livelihoods and other socioeconomic variables (SE\&)]. Graphical summary, cumulative from TAR (left column) to AR4 (middle column) and AR5 (right column) across IPCC regions. South America row includes impacts located in Central America. Size of circles represents the amount of evidence, from zero to emerging, moderate, solid and extensive. 
Despite the global scope of observations and improved coverage for tropical and southern hemisphere regions, geographical imbalances remain (Figure 4.1). Evidence reported in the TAR is almost exclusively related to effects in mid to high northern latitude regions. These regions are still dominant during the AR4, with extensive evidence for physical systems and solid or extensive evidence for ecosystems limited to North America, Europe and the Arctic. In the AR5, evidence from tropical and Southern Hemisphere regions is further substantiated; solid evidence is reported for effects on terrestrial ecosystems in Australasia and Africa as well as for marine ecosystem effects around Australasia and Small Islands.

The strong representation of effects related to temperature-mediated change in snow and ice in the list of observed impacts is one of the reasons for the higher number of observed effects reported from temperate and boreal regions compared to tropical and subtropical regions. Similarly, as changes in spring phenology are amongst the earliest and most widely studied indicators of climate change, the absence of pronounced, temperature-related seasons is one of the reasons for the weaker evidence base for terrestrial ecosystems in low latitude regions. In addition, seasonality and ecosystem processes in tropical and subtropical climates are controlled by precipitation rather than by temperature, and past trends in rainfall regimes in most regions are still less conclusive than those for temperature. Due to the high variability of local rainfall, advances in the attribution of impacts to precipitation changes require the availability of reliable long-term monitoring records. The lack of long-term data and extensive monitoring records, along with less developed research capacities and issues of accessibility of data and literature in languages other than English for international scholars, is likely the most important reason for the weak evidence base for many developing countries (Rosenzweig and Neofotis 2013).

\subsection{Status of knowledge}

Recent changes in climate have caused a widespread impact on both natural and human systems worldwide. Below, the major effects of recent climate change on broad spatial scales are highlighted, followed by a more detailed discussion of some of the key new findings of IPCC'S AR5. This discussion covers marine ecosystems and fisheries, heat and drought induced tree mortality, wildfires, impacts of rising sea levels and impacts observed in the Arctic, including effects on the livelihood of Arctic indigenous peoples.

Table 4.3 summarizes the extensive impacts of recent climate change, defined as impacts that have been attributed to climate change as a major driver with at least medium confidence at a continental scale, or with high confidence for a subcontinental region of substantial size in the AR5. Such substantial impacts across broad areas are documented for species range shifts and phenology 
changes in both marine and terrestrial ecosystems, large-scale vegetation changes in the Arctic Tundra, increased mass bleaching and mortality in tropical reef building corals, enhanced glacier melt and mountain and lowland permafrost thaw, as well as changes in patterns of river run-off related to glacier melt, permafrost thaw and changes in snow regime (Table 4.3). With the exception of changes in river flow, all these impacts have also been attributed to climate change globally.

Table 4.3: Extensive impacts of climate change across regions, extracted from Cramer et al (2014). Extensive impacts (dark squares) are defined here as impacts that have been attributed to climate change as a major driver with at least medium confidence at a continental scale, or with high confidence for a subcontinental region of substantial size. Changes in marine ecosystems exclude the effects observed for tropical coral reef systems, as these are singled out as a separate category. The South America column includes impacts located in Central America. The global assessment is supplemented with information from the marine, coastal, and terrestrial ecosystems chapters of IPCC's WGII AR5 (Pörtner et al 2014; Settele et al 2014; Wong et al 2014).

\begin{tabular}{|c|c|c|c|c|c|c|c|c|c|}
\hline & Africa & Asia & $\begin{array}{c}\text { Austral- } \\
\text { asia }\end{array}$ & Europe & $\begin{array}{l}\text { South } \\
\text { America }\end{array}$ & $\begin{array}{l}\text { North } \\
\text { America }\end{array}$ & Arctic & $\begin{array}{l}\text { Small } \\
\text { Islands }\end{array}$ & Global \\
\hline \multicolumn{10}{|l|}{ Marine ecosystems } \\
\hline Coral bleaching & & & & na & & na & na & & \\
\hline \multicolumn{10}{|l|}{ Terrestrial ecosystems } \\
\hline Glacier melt & & & & & & & & na & \\
\hline Permafrost thaw & na & & na & & na & & & na & \\
\hline River flow change* & na & & na & & & & & na & \\
\hline \multicolumn{10}{|c|}{ *Snow and glacier/permafrost fed rivers } \\
\hline \multicolumn{4}{|c|}{$\begin{array}{l}\text { Extensive impact, i.e. impact documented with at } \\
\text { least medium confidence in a major role of } \\
\text { climate change at continental scale, or high } \\
\text { confidence for a very large sub region }\end{array}$} & \multicolumn{2}{|c|}{$\begin{array}{l}\text { Evidence available, but } \\
\text { below specified } \\
\text { thresholds for } \\
\text { extensive impact }\end{array}$} & & \multicolumn{2}{|c|}{$\begin{array}{l}\text { Not applicable (na) } \\
\text { or no assessment } \\
\text { provided in AR5 }\end{array}$} & \\
\hline
\end{tabular}

The extensive documentation of effects on the marine ecosystem is one of the most prominent new features of IPCC's AR5 (Figure 4.1). Changes in species abundance and distribution consistent with recent climate trends have been shown for many marine species, including fishes, invertebrates and phytoplankton in all ocean basins (Poloczanska et al 2014). The improved representation of marine ecosystems compared to earlier reports is based on a substantial set of additional publications since AR4, but also on the joint effort of the research community to better integrate available knowledge (Richardson et al 2012) and a meta-analysis resulting from that process (Poloczanska et al 2013). Evidence documenting the sensitivity of marine organisms to declining $\mathrm{pH}$ values is abundant (Kroeker et al 2013). Emerging evidence of the impacts of anthropogenic ocean acidification in field data, such as observed shell thinning in planktonic foraminifera (Moy et al 2009) and southern ocean pteropoda (Bednaršek et al 2012) constitutes another central new finding of IPCC's AR5.

Climate change-induced shifts in the composition of fishery catches have been documented globally (Cheung et al 2013). However, the role of climate change in changes in fish stock and fisheries is currently small compared to the more dominant roles of pollution, exploitation and technological development (Brander 2010). While AR5 identifies expected future effects on global fisheries as a 
key risk for coastal communities (IPCC 2014d), effects of past changes in climate on economic outcomes related to fisheries or the livelihoods of fishers has not yet been documented in research literature.

The effect of rising temperatures on forest biomes through drought and heat stress, pest infestations and wildfire is another key area of concern where evidence of the observed effects is now robust. In their comprehensive review of heat and drought-related tree mortality, Allen et al (2010) conclude that recent climate change drives observed drought and heat-related mortality for at least some of the world's forest ecosystems. More recent studies on major forest biomes (Peng et al 2011; Gonzalez et al 2012) and an analysis of drought sensitivity of forests (Williams et al 2012) support this hypothesis, although formal attribution is still lacking (Settele et al 2014).

A signal of climate change in the frequency of wildfires and the area burnt has been documented in most world regions, though no global assessment is available. Signatures of observed climate change are detectable for an increased fire weather risk in Australia (Clarke et al 2013), unprecedented wildfires in the Arctic tundra (Mack et al 2011) and changes in wildfire activity, fire frequency, extent, duration or area burnt in Portugal (Costa et al 2011), Greece (Koutsias et al 2012), Spain (Pausas and Fernández-Muñoz 2011; Cardil and Molina 2013), Canada (Gillett 2004; Girardin et al 2013), the US (Westerling et al 2006) and the Kilimanjaro region (Hemp 2009). The latter are also emerging for the Amazon (Alencar et al 2011; Víctor H. Gutiérrez-Vélez et al 2014). However, determining the relative effect of climate change in damages caused by wildfire is still difficult due to the extremely complex relationships between fuel load, ignition, fire risk management, land-use change, other human factors and weather for the development of (large) fires as well as the role of response strategies, settlement patterns, exposed values and populations for subsequent loss and damage.

Coastal degradation, flooding and other impacts of rising sea levels are a fundamental concern for adaptation planning. Extremely high water levels cause coastal degradation and damage due to flooding. Extreme water levels have increased worldwide since 1970, with a major contribution of mean sea level rise attributed for most locations (Menéndez and Woodworth 2010). Still, studies that attribute the impact on coastal ecosystems and communities to climate change are lacking. Disentangling the contributions of different drivers, such as subsidence, beach mining, changes in sediment supply and the effects of climate change to local sea level rise, is often very difficult. Equally, estimating the relative role of local sea level rise as opposed to coastal development, settlement patterns or flood remediation at a specific location remains a central challenge that often precludes attribution (Nicholls et al 2009; Syvitski et al 2009). However, detailed knowledge utilized in the context of risk assessments and adaptation planning (Ranger et al 2013; National Research 
Council 2014) suggests that the absence of studies documenting past effects may be due to a gap in research rather than a problem of data availability, at least for certain regions.

As in earlier IPCC reports, the Arctic emerges as a hotspot of observed impacts in AR5. Indeed, several authors claim that a regime shift is already occurring in the Arctic (Duarte et al 2012; Jeffries et al 2013). Effects include a biome shift in the Arctic tundra and other terrestrial ecosystem effects, shifts in the abundance and conditions of marine mammals and fish, and changes in marine and terrestrial primary productivity (Post et al 2009; Wassmann et al 2011). These changes extend to related impacts on the livelihood of indigenous peoples (Arctic Council 2013).

There is increasing evidence from a variety of other human systems, most prominently food production and to a lesser extent human health, though in most cases, climate change currently plays a minor role compared to other factors. Detected impacts on human systems and some areas of very dynamic research in this field are discussed further in the next section.

\subsection{A closer look at human systems}

IPCC'S AR5 reports on the emerging impacts of climate change on a variety of humans systems. However, for many important climate sensitive sectors and systems, the effects of recent climate change are poorly studied and documented. Examples include energy supply and demand, forestry, water and waste management, transportation, tourism, and livestock and horticulture. Interestingly, many of these sectors are at the centre of early adaptation actions (Berrang-Ford et al 2011; Ford et al 2011). A recent report taking stock of the relevant econometric information available in the US points out a dramatic lack of such data and large gaps in our knowledge of how important sectors react to changes in climate (Houser et al 2014). Below is a brief summary of the status of knowledge for observed impacts of climate change on the livelihoods of indigenous peoples, food production and human health, followed by a discussion of two very dynamic areas of research: the impact of climate change on occupational health and labour productivity, and the relationship between climate and violent conflict.

\subsubsection{Livelihoods of indigenous peoples}

The impact on Arctic indigenous peoples is reported in both IPCC's TAR and AR4, and is most extensively documented for Arctic North America. Observations include the effects of changing sea ice regimes for travel and hunting, changes in snow regimes and increasing rain-on-ice events for reindeer husbandry, and the impact on traditional food conservation and storage (Beaumier and Ford 2010; Ford and Pearce 2010; Arctic Council 2013). Impacts of changes in permafrost on inland indigenous settlements have also been reported (Alexander et al 2011; Crate 2013; Rosenzweig and 
Neofotis 2013). Much of the underlying evidence is made available through exemplary efforts by sub-organizations of the Arctic Council to facilitate the archiving of traditional knowledge and create community-based monitoring datasets. Exchange between scientists and indigenous communities enabled the synthesis of available knowledge in regional assessments (ACIA 2005; Arctic Council 2013; Eamer et al 2013). Individual studies also address impacts of climate change on indigenous peoples in other regions (Marin 2010; McDowell and Hess 2012), often in the context of adaptation to multiple stressors and local perceptions of climate variability and change. However, they usually do not focus on a systematic evaluation of the role of climate change compared to other factors in historic records and consequently fall short of the requirements of impact attribution analysis (see Chapter 2).

\subsubsection{Food production}

Observed effects of elevated temperatures on staple crops and some other food products, such as wine grapes and fruits, are discussed in IPCC's AR4, but the breadth and depth of coverage increases substantially in AR5. A significant advance over the last decade is the separation of the impact of climate change from agronomic factors in empirical studies and the increasing focus on the influence of extreme temperatures on yields (Auffhammer and Schlenker 2014). Global analyses by Lobell and co-authors show that recent climate trends have reduced yield growth for staple crops (Lobell and Field 2007; Lobell et al 2011b). Other pioneering papers reveal the role of aerosols and monsoon patterns for rice yields in India (Auffhammer et al 2006; Auffhammer et al 2012) and of observed climate trends for staple crop yields in China (Tao et al 2006; Tao et al 2012). Evidence of climate change impacts on crop yields is now available for most world regions (Magrin et al 2009; Schlenker and Lobell 2010; Brisson et al 2010). The sensitivity of crop production to extreme temperatures has been shown for several crops and locations based on historic data (Schlenker and Roberts 2009; Lobell et al 2011a; Lobell et al 2013), however, these studies do not extend the analysis to include an attribution of past effects. Other documented effects include positive yield effects for crops in northern latitudes (Gregory and Marshall 2012; Meng et al 2013) and a negative impact on grapes (Webb et al 2012; Camps and Ramos 2012). Evidence is also emerging for the effect of climate change on coffee growth (Jaramillo et al 2013). Documentation of observed climate change effects on elements of the food systems other than production of staple crops is virtually absent from the scientific literature.

\subsubsection{Human health}

Human health can be impacted by climate change both directly and mediated through environmental effects. Empirical literature mostly focusses on health effects associated with 
exposure to extreme temperatures (Deschenes 2013). The current impact of climate change on human health is not well-quantified, but is estimated to be relatively small compared to the impact of other stressors globally (Smith et al 2014). Recent studies have attributed increases in heatrelated mortality in Australia (Bennett et al 2013) and Sweden (Oudin Åström et al 2013) and shifts in mortality distributions for England and Wales (Christidis et al 2010a) to climate change. Evidence is abundant for the health-related effects of individual extreme events. However, attributing such events and their consequences to climate change still remains a challenge (Hulme 2014; see Chapter 3). There is a dearth of studies examining the relationship between climate, weather and health in middle and low income countries. In some cases, local changes in temperature and rainfall likely have altered the distribution of water-borne illnesses and disease vectors, such as dengue (Earnest et al 2012; Li et al 2013) or malaria (Chaves and Koenraadt 2010; Omumbo et al 2011). Due to public health responses and the general complexity of the issue, the impact detection framework is difficult to apply in the context of human health.

\subsubsection{Occupational health and productivity}

Higher temperatures, and in particular more frequent or severe heat waves, compromise the ability to carry out physical work in exposed conditions. The impact of climate change on occupational health is of particular relevance to many low and medium income countries located in the tropics and subtropics (Kjellstrom et al 2009). The Wet Bulb Globe Temperature (WBGT) index estimates the environmental contribution to heat stress through a combination of air temperature, radiant heat, air movement and humidity. This provides a basis to assess heat stress in the workplace. Based on a reanalysis of WBGT trends, an increase in heat stress between 1975-2000 has been documented for South Asia, the southern US and parts of Central America (Hyatt et al 2010) and a decrease in fully workable days during summer in Delhi has also been found (Dash and Kjellström 2011). Based on a reanalysis of historic global temperature data, Dunne et al (2013) estimate that over the past few decades, environmental heat stress has reduced global labour capacity to $90 \%$ in peak months. However, they do not separate out the contribution from climate change.

In a panel analysis of economic data from 28 Central American and Caribbean countries, Hsiang (2010) finds that short-term increases in temperature are related to losses in economic output across many sectors. Dell et al (2012) find negative effects of temperature on growth rate and absolute economic output for low income countries, based on a panel analysis of 125 countries for the period 1950-2003. Both studies stress that these results extend beyond the agricultural sector and both suggest the effects of extreme heat on labour productivity as a likely explanation. 


\subsubsection{Conflict}

No studies directly attribute any form of civil conflict or violence to recent climate change. However, several studies find an association of climate with spatial patterns of conflict, based on significant correlations between mostly inter-annual variability in temperature and rainfall and some measure of conflict identified in regional or global data sets (Hsiang et al 2013; O'Loughlin et al 2014a). The first of these studies (Burke et al 2009), claiming that warming increases the risk of civil war in Africa, spurred a lively and ongoing debate on the validity of the approach used and the interpretation of its findings. Each of the following publications sparked a series of letters between the disagreeing parties and follow-up publications are beyond the scope of this review. Solow (2013) identifies disciplinary differences as the main line of conflict in this debate. For example, political scientists will highlight the specific circumstances of each country and conflict, while 'black box' statistical models are prone to ignore these to a certain extent. Besides the central argument of how far causation can be inferred from correlation, technical issues such as appropriate data resolution, choice of model and the treatment of confounding variables have also been the subject of debate (Burke et al 2014; O'Loughlin et al 2014b). However, that weather and, most notably, climate extremes mediated through their impact on resources, employment, livelihood, well-being and governance are probably implicated in the development and escalation of conflicts is undisputed.

\subsection{Conclusion and outlook}

The review of the three most recent IPCC assessment reports (TAR, AR4 and AR5) clearly shows how evidence documenting observed climate change impacts has expanded and substantiated over time.

The Arctic region is a hot-spot of climate change impacts. A wide range of impacts is also documented for natural and human systems across world regions and oceans. Still, a substantial part of the observational evidence originates from a specific set of impacts, such as glacier melt or phenology changes in temperate and boreal ecosystems. In spite of the robust evidence in some human sectors and systems, there are still large gaps in our understanding of how important sectors of human society respond to past climate change, even in the form of econometric (or other) data on sensitivity. Lack of monitoring is a key reason for the weak representation of some regions, most notably Africa and Small Islands. Other gaps in knowledge, such as the lack of attributed impacts of sea level rise, could be addressed by research dedicated to attribution of impacts of climate change.

Despite some differences in the approaches taken for the analysis and presentation of impact detection and attribution, the findings of the IPCC'S TAR, AR4 and AR5 are broadly consistent, and the pattern that emerges from the synopsis of the three reports appears to be robust. The approach chosen by the AR5 facilitates the structured assessment of impacts that were acknowledged in 
earlier reports, but excluded from formal analysis, such as impacts on the livelihoods of indigenous peoples.

For many climate change impacts on human and managed systems detectable today, climate change plays a relatively minor role in comparison with other drivers of change. Accelerating rates of climate change and the predicted crossing of important climate thresholds could modify this picture substantially in the future. To assess the effects of climate change, databases and research designs to study and monitor effects of adaptation will become increasingly important. 


\title{
5 Attributing observed climate change impacts to human influence
}

\begin{abstract}
A wealth of evidence documents the widespread impact of recent regional climate change on natural and human systems. However, studies explicitly addressing the role of anthropogenic forcing of the climate for such observations are still rare. This chapter extends the recent assessment of observed impacts of regional climate trends provided by the IPCC's Fifth Assessment Report by adding a climate attribution step investigating the role of anthropogenic forcing for the climate trends that contribute to the observed effects. Results show that for documented effects related to atmospheric and ocean temperature change, the uncertainties surrounding the attribution of observed impacts to climate change are higher than those related to the attribution of recent climate change to human influence. In contrast, the signature of human influence remains hardly detectable for changes in precipitation. Combining the impact attribution and the climate attribution steps in a multi-step attribution assessment shows that anthropogenic forcing of the climate system has a major role for approximately $42 \%$ of the reported impacts with at least medium confidence. This share rises to $50 \%$ when only temperature-related effects are considered and to $75 \%$ of the cases for detection of at least a minor anthropogenic effect in temperature-related impacts. Therefore, this analysis confirms earlier statements that anthropogenic climate change is having a discernible effect on natural systems worldwide; it even extends this statement to include human and managed systems. However, it is also clear from the comparison of the impact and climate attribution steps for individual impact assessments that high levels of confidence in climate attribution cannot be inferred from high levels of confidence in impact attribution, and vice versa.
\end{abstract}

This chapter corresponds to a manuscript that is currently prepared for submission by Hansen, $\mathrm{G}$ and Stone, DA. The method applied is documented in Annex A, and a detailed list of impacts analysed and corresponding results is provided in Annex B. 


\subsection{Introduction}

In its Fourth Assessment Report, the Intergovernmental Panel on Climate Change (IPCC) states that "it is likely that anthropogenic warming has had a discernible influence on many physical and biological systems" (IPCC 2007b). This statement is based on a synthesis of local and regional studies that shows a high level of spatial agreement between regions of significant anthropogenic warming across the globe and the locations of significant observed changes consistent with warming in many natural systems (Rosenzweig et al 2007; Rosenzweig et al 2008).

The IPCC's Fifth Assessment Report (AR5) investigates the detection and attribution of observed impacts to recent changes in climate (contribution of IPCC working group II, Cramer et al 2014) and the detection and attribution of observed changes in global climate to anthropogenic forcing (contribution of IPCC working group I, Bindoff et al 2013) but fails to provide an assessment of the relevance of one for the other. In their summary for policymakers, the working group I (WGI) contribution to the AR5 highlights that "it is extremely likely that human influence on climate caused more than half of the observed increase in global average surface temperature from 1951-2010. There is high confidence that this has warmed the ocean, melted snow and ice, raised global mean sea level, and changed some climate extremes, in the second half of the 20th century" (IPCC 2013a). The corresponding document of the IPCC working group II (WGII) states that "in recent decades, changes in climate have caused impacts on natural and human systems on all continents and across the oceans" (IPCC 2014d). Though the idea suggests itself, it has not been systematically explored whether or not the observed climate changes that have been attributed to human influence have caused impacts or how the documented effects attributed to recent changes in climate are related to anthropogenic climate change.

A primary reason for this gap is the weak representation of studies analysing the full chain of impact from human emissions to observed effects - so called 'end-to-end studies' - in the available literature (Gillett 2004; Barnett et al 2008; Hidalgo et al 2009; Christidis et al 2010a; Marzeion et al 2014). Reasons for the lack of end-to-end studies include a mismatch of spatial scales between impact studies that are mainly local and the general circulation models used for the attribution of climate change to emissions, the insufficient length of many observational impact records and other issues such as incompatible disciplinary approaches or simply other research priorities (Hegerl et al 2010; Parmesan et al 2013; Stone et al 2013; see also Chapter 2). Despite attempts to establish common standards and approaches to the detection and attribution challenge early in the AR5 assessment cycle (Hegerl et al 2010), the attribution of impacts to anthropogenic forcing of the 
climate system is not systematically explored across the broad scale of natural, human and managed systems within the AR5.

Instead, in the AR5 WGII chapter on "detection and attribution of observed impacts", Cramer et al (2014) provide a synthesis of impacts of long-term regional trends in temperature, precipitation, sea ice extent, sea level rise and ocean acidification. They explicitly consider the contribution of nonclimatic drivers to these observed changes, such as land use, pollution and technological development. Their assessment is built on expert judgement, drawing on the majority of the AR5 WGII chapters. It provides information about both the detection of a climate change effect in observations and the role of climate change compared to other drivers of change (Stone et al 2013). Following the AR5 uncertainty guidance (Mastrandrea et al 2010), confidence is expressed based on type, amount, quality and consistency of available evidence and the degree of agreement. A central result of the analysis consists in a set of tables summarizing regional impacts for five major impact categories - cryosphere, hydrology, terrestrial ecosystems, marine and coastal ecosystems, and human and managed systems (Tables 18.5-18.9 in Cramer et al 2014). In these tables, several characteristics associated with each of the observed impacts are stated explicitly: the most relevant climate change variables, the assumed baseline behaviour, other relevant drivers of change, the respective role (at least minor, or major) of climate change in the overall change observed and the level of confidence for both the detection of an effect of climate change and the attribution of the observed impact to climate change. A major role of climate change is ascribed "if the past behaviour of the system would have been grossly different in the absence of the observed climate change" (Cramer et al 2014). For example, widespread permafrost degradation, especially in the southern Arctic region, is attributed to regional warming with high confidence and a major role is assigned to climate change. In contrast, while it is stated with medium confidence that warming has had a detectable negative effect on global maize and wheat yields, the role of the warming trend is assessed to be minor compared to that of other factors, such as agronomic development.

While Cramer et al (2014) provide a comprehensive assessment of the observed impact of recent changes in climate, they do not discuss the role of anthropogenic climate forcing for this impact. One way to evaluate that role comprises a comparison of the historic climate conditions in the region specified in the impact assessment (for example, warming in the southern pan-Arctic permafrost region) with a hypothetical 'natural' climate in the same region, i.e. a climate without anthropogenic forcing derived from climate modelling experiments.

This chapter builds on the assessment of the role of recent climate trends for observed impacts across natural and human systems (hereinafter impact attribution) by Cramer et al (2014) and provides an assessment of the role of anthropogenic forcing in the observed changes in climate 
(hereinafter climate attribution) that are reported to drive specific impacts therein, explicitly stating the level of confidence in the assessment. The analysis applies a new method that systematically assesses the influence of anthropogenic forcing of the climate system for observed regional climate trends, documented in Annex A. Based on the results, some insights into the factors controlling the different patterns of confidence for the impact and the climate attribution steps are provided and impacts that score high in both assessments are highlighted. The two investigations of impact attribution and climate attribution are then aggregated in order to provide a multi-step assessment of the role of anthropogenic forcing for these impacts (hereinafter combined attribution), thereby narrowing the gap left by the AR5.

\subsection{Methods}

The central part of this analysis consists in the application of a climate attribution confidence algorithm to the regional climate trends that are reported to cause impacts specified in Cramer et al (2014). The climate attribution confidence algorithm (see Annex A) provides confidence values for assessing the role of anthropogenic forcing in observed changes in climate for specific regions, periods and climate variables. Figure 5.1 presents a schematic of the steps covered by the algorithm; an overview of the process, data and individual steps of the algorithm is given below in Section 5.2.3 and the full description including calculations used for the factors corresponding to each step is provided in Annex A.

The full analysis comprises the following stages:

- Identification of the relevant climate variables and seasons for each impact;

- Specification of spatial characteristics of for each impact;

- Extraction of corresponding spatially explicit climate data (gridded observational data and climate model output from simulations representing historic and hypothetical 'natural' climates) for the period, area and climate variable specified;

- Regression analysis of the observed datasets against modelled climate response for the same region and period;

- A series of tests that measure the adequacy of the observational input and agreement between observed and expected climates based on the regression coefficients;

- The mapping of the numerical output of the test series onto the scale of qualitative confidence levels; and

- The combination of the impact attribution and climate attribution steps into a multi-step attribution analysis assessing the impact of anthropogenic climate change. 


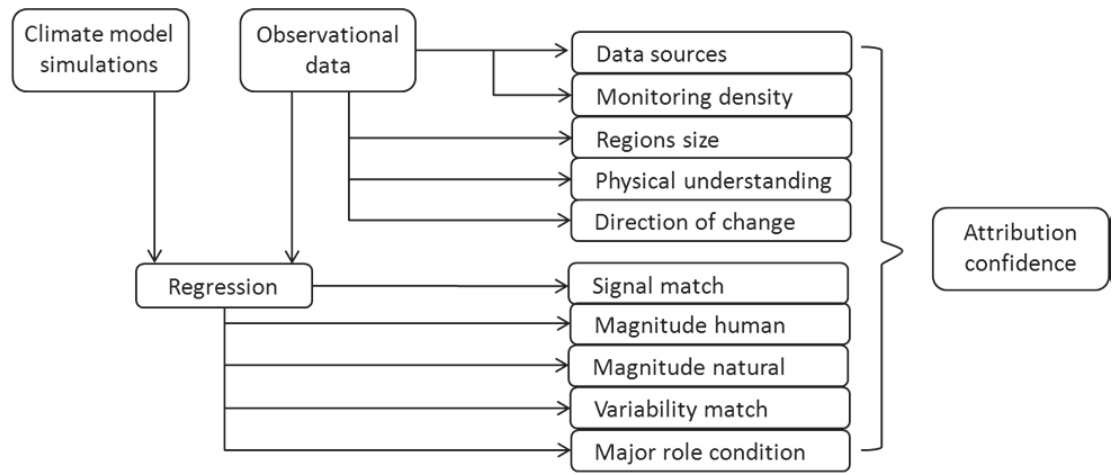

Figure 5.1: Schematic of the algorithm for estimating the level of confidence in the detection of a climate response to anthropogenic drivers and attribution of a major role. An initial metric of complete confidence that depends on the number of available data sources is degraded according to tests based on various criteria. The resulting values are converted into confidence levels ranging from 'none' to 'very high'.

The resulting set of confidence values for the attribution of a major or at least minor role to anthropogenic forcing in driving that trend is compared with the impact attribution confidence for each case (5.2.4). The role of impact systems, spatial extent and climate variables for the level of confidence is analysed for climate and impact attribution respectively. Finally, the impact attribution and climate attribution assessments are combined to provide a multi-step assessment of confidence in the role of anthropogenic climate change in observed climate-related effects.

The analysis is limited to impacts driven by long-term temperature changes over land and in the ocean, including ocean temperature as a proxy for sea ice, and changes in precipitation. These impacts form the majority of the impacts reported in Cramer et al (2014).

\subsubsection{Stratification of impact assessment data}

The original database consists of all regional assessments from Tables 18.5-18.9 complimented by some global statements taken from Section 18.6 in Cramer et al (2014). It includes 123 assessments, 25 of them representing impacts driven by more than one changing climatic factor. Selection restrictions eliminate 19 assessments, yielding 104 assessments and reducing the number of multiple assessments to 14 (see Figure 5.2). Entries are omitted from the original list for several reasons, including:

- Drivers not included in the design of the climate attribution assessment, such as sea level rise, $\mathrm{CO}_{2}$ concentration, extreme precipitation and ocean acidification;

- Unclear spatial specification that could not be resolved; and

- Lack of a clearly specified direction of climate change in the assessment and supporting literature (e.g. changing rainfall patterns). 
We focus on the metric defined as attribution in Cramer et al (2014). The distribution of assessments stating a major versus minor role of climate change is approximately constant between the final and initial lists of effects (77\% and $79 \%$ major role respectively). One outstanding change consists in the reduced number of marine assessments in the final (21) compared to the original (30) database, which is mainly due to the difficulty to assign appropriate spatial characteristics to some of the processes described, for example impacts pertaining to migrating sea birds.
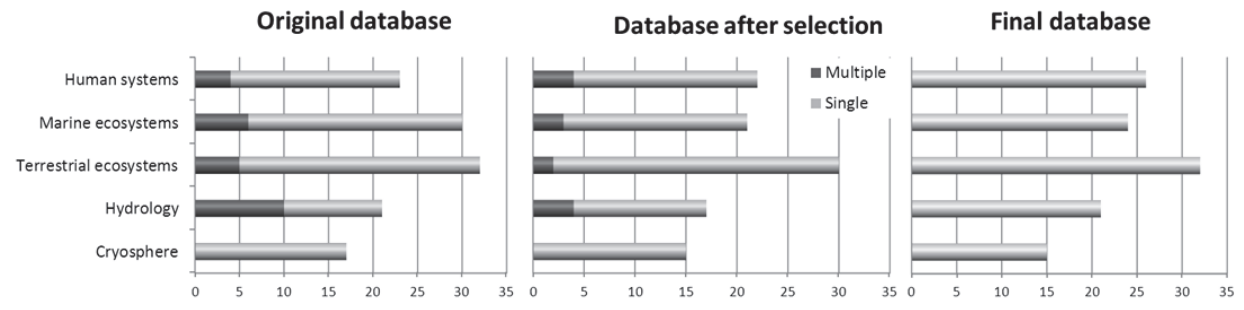

Figure 5.2: Number and distribution of assessments in the original (left panel) and interim database (centre panel) across systems, differentiated by the number of climate drivers. 'Single' refers to assessments that state only one climate driver, such as warming, while 'multiple' refers to assessments that state more than one climate driver, such as warming and reduced precipitation. The right panel shows the distribution of the 118 assessments in the final database that result from splitting multiple driver assessments in the interim database into two individual assessments.

During the climate attribution step, the individual climate drivers are to be evaluated separately for each impact assessment. Due to the exclusion of some climate drivers, the fourteen remaining paired climate drivers are limited to air temperature over land and precipitation, air temperature over land and sea ice extent (with ocean temperature serving as a proxy for sea ice, which is not well-reproduced in models), and air temperature over land and ocean surface temperature. For simplification, the initial database is then transformed by adding a separate entry for each of these assessments, resulting in a total of 118 entries.

\subsubsection{Specification of spatial extent and assessment period}

In order to attribute the climate trend contributing to the observed impacts to anthropogenic forcing, appropriate spatial and temporal characteristics must be assigned to each assessment. For example, in the case of spring phenology changes due to atmospheric warming across the US, the spatial extent might be defined as the continental US territory and the relevant period as spanning February to May, the late winter and early spring months in that territory. The areas that correspond to an impact are defined by a combination of land-sea boundaries, administrative boundaries and polygon shapes based on a $0.5^{\circ}$ longitude-latitude grid, roughly sketching prominent geographical features where appropriate (see Figure 5.3). Given the coarse resolution of the climate models and 
observational data sets that are to be used for climate change attribution, the quality of the spatial information derived is deemed appropriate. For cases where information in Cramer et al (2014) appears ambiguous, papers and report sections cited in support of the statement are reviewed in order to find the most appropriate regional specification; sometimes assumptions are required beyond what is explicitly stated by the original authors. In cases where a summary statement applies across several affected sub-regions (e.g. glaciers across Europe) and the geographic area of concern is discontinuous or too far apart to be merged into a larger shape without losing the connection to the climate information, the spatial area is split into sub-regions and the impact statement is treated as valid for the collection of these sub-regions. For some cascading effects, the primary climate driver occurs over an area different from that where the impact is observed. Here, the spatial extent is used that corresponds to the primary climate effect. For impact statements related to river flow or discharge, the entire river basin is taken as a basis for climate attribution. The specific areas considered are limited to those where an effect could realistically have taken place. For example, the spatial extent of permafrost degradation in the Arctic is confined to areas of continuous and discontinuous permafrost in the low Arctic (Figure 5.3).
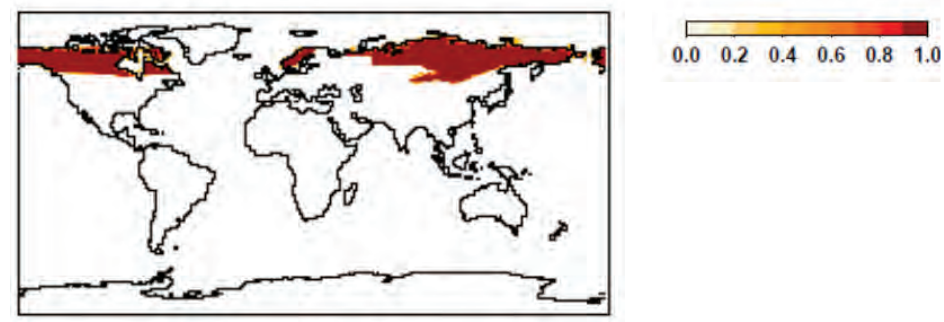

Figure 5.3: Example for the spatial specification step. Shown here is the aforementioned area affected by permafrost degradation in the Arctic. Grid cells that are only partly included in the affected area are weighted according to the share of the cell that is affected, illustrated by colours corresponding to the respective share on the scale.

In many cases, information on the overall extent of the respective areas is included in the underlying literature. Where information is not readily available from cited sources, estimates are based on various sources, such as interactive maps (e.g. the Arctic portal interactive map (www.portal.intermap.com) for polar Issues, the World Glacier Inventory (http://nsidc.org/data/glacier_inventory), spatial information from government agencies (e.g. www.nationalatlas.gov) and scientific publications. Administrative boundaries are taken from Natural Earth v1.4.0 (http://www.naturalearthdata.com). Unless the coordinates can be extracted from cited references, mapping is based on the National Geographic Atlas of the World, $7^{\text {th }}$ edition and Google Earth. 
With a few exceptions, Cramer et al (2014) are not specific about the time period that they reference in Tables 18.5-18.9, noting only that statements relate to the past several decades / recent climate change. The assessment itself is designed to identify impacts that are in line with current climate trends and that are expected to continue should those trends continue or strengthen. However, a clear timeframe has to be specified for the analysis of climate attribution, so the default 40 year period from 1971-2010 is chosen as a reference. A sensitivity analysis is run to test the robustness of the assessment against the start year, end year and overall length of the period considered. For table entries where time periods are explicitly stated or the underlying papers and chapter subsections indicated a different - usually longer - time span, these periods are analysed separately (see 5.3.2.4); however, the overall assessment is based on the default period.

\subsubsection{Climate attribution assessment}

Changes in climate are not necessarily caused by human influence. They can also be due to naturally occurring acts of external forcing, such as solar variations and explosive volcanism or internal variability of the climate system. The response of the climate system to external forcing can be simulated by global circulation models (GCMs). The algorithm applied in the climate attribution step is developed around the comparison of observed variations in the climate with expectations of how the climate should have changed, as expressed in ensembles of GCMs (Hegerl et al 2010; Hegerl and Zwiers 2011).

The algorithm for estimating confidence in the attribution of a given aspect of climate change to human influence use various combinations of the following inputs in a series of tests:

- Information about the relevant climate variable(s), direction of the observed change, specification of the relevant season and spatial extent (described in previous section);

- $\mathrm{N}_{\text {obs }}$ gridded observational products. By using multiple data sets, measurement error and the uncertainty in calculating a regionally and seasonally averaged estimate from instantaneous point measurements can be considered to some extent;

- Simulations of the climate system from Nmod models, driven with all known forcing factors, both anthropogenic and natural, and with natural forcing only. For dynamical climate models, multiple simulations, producing different possible weather trajectories for each model, allow a more accurate estimate of the model's response signal; and

- A large number of years of simulations of dynamical climate models with no variations in external drivers beyond the diurnal and annual cycle to reproduce internal natural variability. 
In addition to the comparison of observed and modelled climates, the adequacy of the inputs to the analysis must also be considered. As described below, this is done by using a number of tests that address, for example, the ability of models to adequately reproduce climate over smaller areas or the amount and quality of observational data available based on the density of the monitoring network.

A metric $c$ is assigned a starting value that depends on the number of data sources available. $C$ is then left unchanged or reduced based on a series of tests that consider the adequacy of the input data sources and the agreement between observed changes and our expectations based on processbased modelling, conducted via a linear regression. $C$ is multiplied with a factor $\mu \leq 1$ for each test. The resulting value $c_{m}$ is then converted into confidence levels ranging from 'none' to 'very high' (Table 5.1). Figure 5.4 illustrates how the climate attribution confidence changes with each test for impacts driven by ocean warming.

Table 5.1: Mapping of the quantitative outcome of the climate attribution algorithm to the qualitative confidence levels.

\begin{tabular}{|l|l|}
\hline Confidence level & Numerical value \\
\hline No confidence & $\mathrm{c}_{\mathrm{m}}=0$ \\
\hline Very low confidence & $0<\mathrm{c}_{\mathrm{m}}<1$ \\
\hline Low confidence & $1 \leq \mathrm{c}_{\mathrm{m}}<2$ \\
\hline Medium confidence & $2 \leq \mathrm{c}_{\mathrm{m}}<3$ \\
\hline High confidence & $3 \leq \mathrm{c}_{\mathrm{m}}<4$ \\
\hline Very high confidence & $4 \leq \mathrm{c}_{\mathrm{m}}$ \\
\hline
\end{tabular}

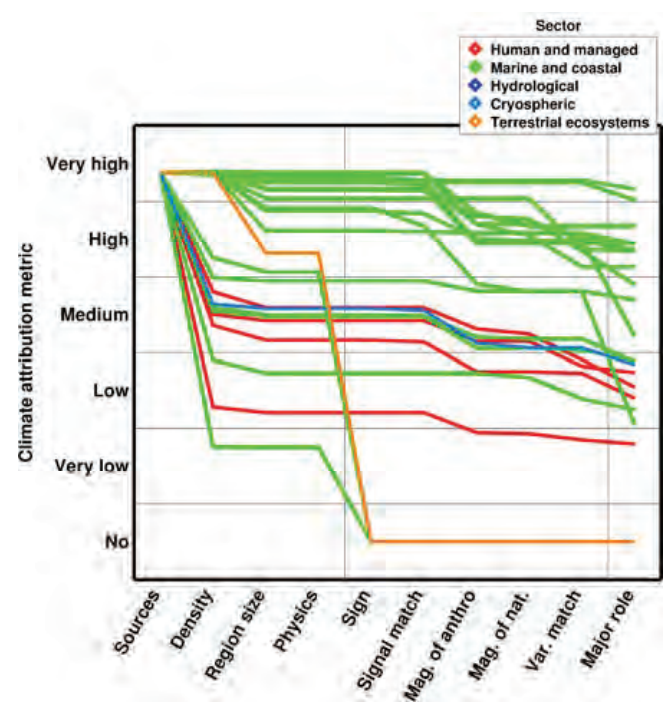

Figure 5.4: Climate attribution algorithm output for all impacts related to ocean temperature. Colour depicts the impact sector corresponding to the respective climate attribution assessment. 
Available climate attribution assessments from IPCC AR5 (Bindoff et al 2013; Niang et al 2014) are used to test the outcome of the algorithm for plausibility. Below, a short description of the individual assessment steps is given; for a full description, including numerical formulations corresponding to each test, see Annex A.

\subsubsection{Adequacy of observational data sources}

Data sources: The observational and prediction products comprise the ultimate sources of evidence; the number of such products is thus the starting point for the confidence algorithm, with more products available increasing the robustness of the evidence base. Table 5.2 lists the gridded observational data products used in this analysis, three for air temperature, four for precipitation and two for sea surface temperature. Climate model simulations used in the analyses in this chapter are taken from the Coupled Model Intercomparison Project Phase 5 (CMIP5) climate model database (Taylor et al 2012). The regression model is estimated for each combination of the $\mathrm{N}_{\bmod }=7$ climate models with available simulations and the $\mathrm{N}_{\text {obs }}$ observational products, that is, 21 regression models for 2 metre air temperature over land, 14 for sea surface temperature and 28 for precipitation. In the interests of both data compression and of focussing on longer timescale variations, 5 year nonrunning averages of these data are examined.

Table 5.2: List of observational data products used for analysis in this chapter. On average, a $1^{\circ}$ longitude by $1^{\circ}$ latitude grid box covers $7900 \mathrm{~km}^{2}$ across the globe, being larger at the equator than at the poles.

\begin{tabular}{|l|l|c|}
\hline Climate variable & Data products & Spatial resolution \\
\hline $2 \mathrm{~m}$ air temperature & CRU TS 3.22 (Harris et al 2014) & $2000 \mathrm{~km}^{2}$ \\
& GISTEMP v6 (250 km land) (Hansen et al 2010) & $63000 \mathrm{~km}^{2}$ \\
& UDel v3.01 (Matsuura and Willmott 2012) & $2000 \mathrm{~km}^{2}$ \\
\hline Precipitation & CRU TS 3.22 (Harris et al 2014) & $2000 \mathrm{~km}^{2}$ \\
& GPCC v6 (Schneider et al 2014) & $7900 \mathrm{~km}^{2}$ \\
& NOAA PRECL (1x1 $1^{\circ}$ (Chen et al 2002) & $7900 \mathrm{~km}^{2}$ \\
& UDel v3.01 (Matsuura and Willmott 2012) & $2000 \mathrm{~km}^{2}$ \\
\hline Sea surface temperature & HadISST1 (Rayner et al 2003) & $7900 \mathrm{~km}^{2}$ \\
& Hurrell (Hurrell et al 2008) & $9200 \mathrm{~km}^{2}$ \\
\hline
\end{tabular}

Monitoring density: The spatial distribution of observational measurements is not uniform; in some areas it may be considered low enough to prevent the development of confidence in their ability to be representative of the regional climate. The adequacy of measurement density is estimated based on the fraction of variance in the time series for a regional climate variable that is accounted for by the given measurement density, building on the method employed in New et al (2000). The station density information from the CRU TS 3.22 (Harris et al 2014) product are used for land temperature and precipitation and that from the HadSST3.1.1.0 (Kennedy et al 2011a; Kennedy et al 2011b) dataset for sea surface temperature. 
Region size: Dynamical climate models have a limited spatial resolution, meaning they are better at reproducing variations in large-scale mechanisms than in ones closer to the resolution size. Furthermore, the smaller-scale characteristics of the anthropogenic drivers of climate change (particularly aerosol emissions) are less well-understood than larger-scale properties. This means there is a priori less confidence in climate model results for smaller regions. Similarly, the accuracy of observational products becomes more sensitive to the interpolation method used at scales around, or smaller than, the station separation. To account for this, the confidence metric is reduced by an amount relative to the region's size.

Climate variable: The basic physical processes behind many aspects of the climate are both well understood and mostly resolved in dynamical models, but this is not the case for some variables. For instance, the microphysical processes that generate precipitation are not simulated in climate models but rather approximated by somewhat heuristic algorithms. In recognition of this, the confidence metric is multiplied by a constant that only depends on the climate variable, effectively reducing confidence for precipitation by one level.

Observed change matches required change: In Cramer et al (2014), impacts are reported to have been caused by a specific observed climate trend. Therefore, it is necessary to confirm that this trend also exists in the observational data sets used in the climate change attribution analysis. Reasons for discrepancies could include ambiguity over time period, regional or seasonal definitions or disagreement between local and global data sets. This step yields zero should at least half of the observation datasets fail to reproduce the direction of change stated in Cramer et al (2014).

\subsubsection{Comparison of observed and predicted climate change}

The analysis method behind much research carried out in recent years regarding the detection and attribution of climate change to anthropogenic forcing applies a linear regression model to compare output from climate model simulations with observed climate changes (Allen and Tett 1999; Bindoff et al 2013).

$\mathrm{X}_{\mathrm{obs}}(\mathrm{t})$ represents variations in an observed climate variable as a function of time $(\mathrm{t}), \mathrm{X}_{\mathrm{ant}}(\mathrm{t})$ represents the expected climate response to anthropogenic external drivers, and $X_{n a t}(t)$ represents the expected climate response to natural external drivers. The regression assumes that responses to climate change are linearly additive, which appears reasonable for temperature and precipitation responses of the magnitude and spatial scale considered here (Shiogama et al 2012), and can be written as (Allen and Tett 1999):

$$
X_{\text {obs }}(t)=b_{\text {ant }} \cdot X_{a n t}(t)+b_{\text {nat }} \cdot X_{\text {nat }}(t)+R(t)
$$


Here $R(t)$ is the residual of the regression and $b_{a n t}$ and $b_{\text {nat }}$ are the regression coefficients estimated such that the variance of $R(t)$ is minimized. This formulation of the regression assumes that the $X_{\text {ant }}$ ( $t$ ) and $X_{\text {nat }}(t)$ responses for the climate model can be perfectly estimated (Allen and Stott 2003). Available climate model data (Taylor et al 2012) generally only cover the $X_{n a t}(t)$ response signal and the $X_{\text {all }}(t)=X_{\text {ant }}(t)+X_{\text {nat }}(t)$ response to the complete combinations of anthropogenic and natural drivers; the regression can thus be written as follows:

$$
X_{\text {obs }}(t)=b_{\text {ant }} \cdot X_{\text {all }}(t)+\left(b_{\text {nat }}-b_{\text {ant }}\right) \cdot X_{\text {nat }}(t)+R(t)
$$

The regression coefficients $b_{\text {ant }}$ and $b_{\text {nat }}+b_{\text {ant }}$ and their uncertainty due to the limited sampling of the observed climate response against the noise of natural internally generated variability of the climate system are estimated using the code available at http://www.csag.uct.ac.za/ daithi/idl lib/detect/. Traditionally, a response to anthropogenic forcing is considered to be detected if $b_{\text {ant }}$ is positive and inconsistent with zero at some level of statistical significance given this sampling uncertainty.

This regression is performed separately for each combination of the $\mathrm{N}_{\mathrm{obs}}$ observation data products and the $\mathrm{N}_{\text {mod }}$ climate model products. For each of the models with available simulations for estimating the response signals, the sampling noise is reduced by averaging across the 3 to 10 simulations available in each case, with $\mathrm{N}_{\text {mod }}=7$ models running a total of 48 simulations for historical and 33 for natural historical climate. The translation of these regression analyses into penalisation of the confidence metric is summarized below. Overall, the penalties emerge from a combination of the fractions of the $\mathrm{N}_{\bmod } \times \mathrm{N}_{\text {obs }}$ regression coefficients that fulfil or fail the test criteria of the respective step and a term that expresses the weight of that step.

Match of signals: This test addresses the question of whether the fingerprint of the anthropogenic response expected by the climate models is really found in the observational data. In terms of the regression, the question is whether $b_{\text {ant }}>0$. This step is the critical test for a climate change detection analysis.

Match of magnitude of anthropogenic climate change: A match in magnitude can be considered an indication that the observed signal analysed in the regression is indeed the predicted signal, rather than, for instance, a response to an ignored driver that happens to closely resemble the predicted response to anthropogenic drivers. Within the regression formulation used here, the question is whether the regression coefficients for the anthropogenic response, $b_{\text {ant }}$ are not inconsistent with 1 . 
Match of magnitude of natural climate change: The above test only concerns the response to anthropogenic drivers. While they are less directly connected to the conclusions of the analysis, it would also help build confidence (or to maintain it) if the observed response to natural drivers is also not inconsistent with the predicted response.

Consistency of autonomous variability: As an extremely non-linear system, the climate generates variability autonomously, whether it is being influenced by external factors or not. If the assumptions behind the regression hold and all important external drivers have been included in the $X_{\text {nat }}(t)$ and $X_{\text {nat }}(t)$ pair, then the residual $R(t)$ from the regression should be indistinguishable from this autonomous variability. A comparison is performed between the $\mathrm{R}(\mathrm{t})$ arising from the regression and the variability in unforced simulations. If the residuals from all $\mathrm{N}_{\text {obs }} \times \mathrm{N}_{\bmod }$ combinations fail the test, then the confidence metric is reduced by up to three levels. As with inconsistencies in the regression coefficients, gross failure of the residual test is a major concern and could reflect unaccounted drivers.

Assignment of major role: Assessment of the role of anthropogenic forcing in observed changes in climate requires a description of the magnitude of the contribution of human influence relative to other factors (Hegerl et al 2010). For this algorithm, it is assessed whether emissions have had a 'major role' in the behaviour of the observed climate, defining 'major role' as cases where the anthropogenic response accounts for at least one third of the temporal variance (see Annex A). Other possible contributors to the variance would be the response to natural drivers, autonomous variability or possible unidentified drivers.

\subsubsection{Combined attribution assessment}

Confidence levels for the impact and the climate attribution side are compared using different measures of aggregation for systems, spatial extent and climate drivers. To enable that comparison, the climate attribution confidence is constructed in the same way as the impact attribution confidence in the IPCC AR5 assessment, i.e. specifying attribution of either a major or at least a minor role. An extended discussion is provided for the impacts assessed to imply a major role of the respective driver for both the climate and the impact aspect. In addition, a tentative assessment of the impacts of anthropogenic climate change is derived by combining the two assessments.

There are two measures that are relevant in order to combine impact attribution and climate attribution into one overarching metric providing information about the level of confidence in the role of anthropogenic forcing for the observed effect (combined attribution): the role attribute (major, minor) for both impact and climate assessment and the respective level of confidence. 
Obviously, the combined attribution metric must be smaller than or equal to the minimum of the two separate assessments. The individual steps might be combined using, for example, a minimum value approach or by multiplication. Given that only bin values are available for the impact confidence, values are coarsely discretised. The maximum difference between the multiplicative approach (see Table 5.3) and the minimum value approach (see Table 5.4) is one level of confidence. Even for the minimum value approach, the combined metric distribution is, on average, less confident than both the individual distributions, because only a small share of the data has identical values for both role and confidence levels.

Table 5.3: Matrix for combining two confidence assessments consisting of discretized bin values, using a multiplicative approach; confidence levels for impact attribution is shown in rows and climate attribution in columns. Grey scale indicates combined confidence, from white (none) to black (very high).

\begin{tabular}{|l|l|l|l|l|l|l|}
\hline & NONE & VERY LOW & LOW & MEDIUM & HIGH & VERY HIGH \\
\hline VERY LOW & & & & & & \\
\hline LOW & & & & & & \\
\hline MEDIUM & & & & & & \\
\hline HIGH & & & & & & \\
\hline VERY HIGH & & & & & & \\
\hline
\end{tabular}

Table 5.4: Matrix for combining two confidence assessments consisting of discretized bin values, using a minimum value approach; confidence levels for impact attribution is shown in rows and climate attribution in columns. Grey scale indicates combined confidence, from white (none) to black (very high).

\begin{tabular}{|l|l|l|l|l|l|l|}
\hline & NONE & VERY LOW & LOW & MEDIUM & HIGH & VERY HIGH \\
\hline VERY LOW & & & & & & \\
\hline LOW & & & & & & \\
\hline MEDIUM & & & & & & \\
\hline HIGH & & & & & & \\
\hline VERY HIGH & & & & & & \\
\hline
\end{tabular}

Given that the confidence metric does not represent a statistical measure in a strict sense, a simple minimum value approach was chosen for the combined assessment. In cases where the roles differ for the impact and the climate attribution assessment, the smaller role is assigned to the combined assessment. A control run using the 'minor role' confidence labels in those cases resulted in slightly higher confidence in only three cases, so the minimum value approach was applied for consistency and simplicity. Cases of multiple climate drivers are treated as separate assessments.

\subsection{Results}

This section briefly introduces the characteristics of the impact attribution assessment from AR5, followed by a more detailed analysis of the results of the climate attribution analysis. A comparison of the two assessments is provided and results of aggregating both steps into a combined attribution assessment are discussed. 


\subsubsection{Impact attribution confidence}

In order to enable an informed comparison of the confidence value pairs for the impact and the climate attribution step, a brief review of the input data selected from Cramer et al (2014) is included here. One of the first things to note is that, on average, confidence levels are higher for natural system effects than for impacts on human or managed systems. Also, the assessment is clustered around 'medium confidence' values, with only $22 \%$ of the confidence values being lower and $28 \%$ being higher, while both 'very high' and 'very low' confidence levels are rare or totally absent. This suggests a filtering mechanism that avoids stating extremes, or possibly the choice of 'major' and 'minor' roles in a way that promotes 'medium confidence' over the other values.

There is a selection bias to begin with, as the assessment focusses on impacts where evidence allows confident conclusions, potentially side-lining many assessments that might have qualified for 'very low confidence' and excluding negatives. This fact is also evident in the much higher ratio of 'major' compared to 'minor' role statements in the impact assessment.

\subsubsection{Major and minor roles and relation to sectors}

The 118 entries in the stratified database (i.e. after filtering as described in Section 2.2) are distributed over human and managed systems (26), terrestrial ecosystems (32 values), marine ecosystems (24 values), hydrology (21 values), and the cryosphere (15 values). Confidence in attribution of impacts to climate trends is highest within the cryosphere, whereas lower confidence and a high share of 'minor role' assessments prevail for human and managed systems. Confidence is more evenly distributed for ecosystems and hydrology, and there are comparatively few minor role assessments, especially for the higher confidence ranges (see Figure 5.5).

The total of 118 values is comprised of 95 major role and 23 minor role assessments; minor role assessments therefore only represent approximately one fifth of the total. Similar to the nearabsence of very low confidence assessments, this may partly be due to the fact that, in a multi-factor environment, a climate change impact will only be detected with confidence in situations where its effect is substantial. This is in keeping with the fact that confidence is generally higher for those assessments that attribute a major role to climate change. 


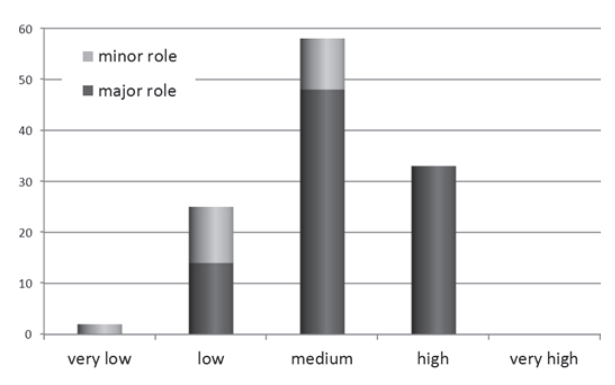

All impact systems
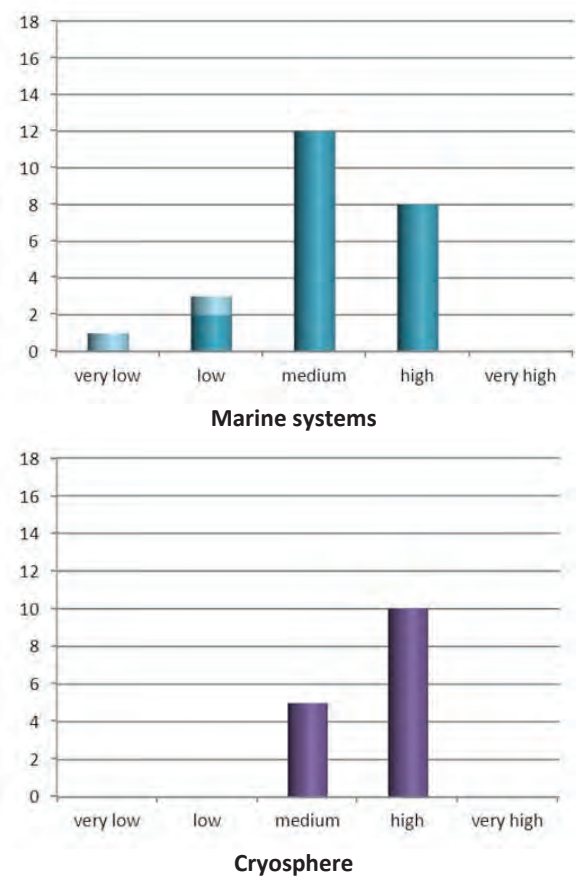

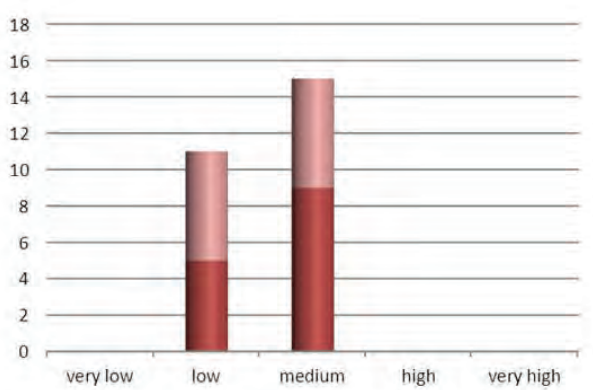

Human and managed systems

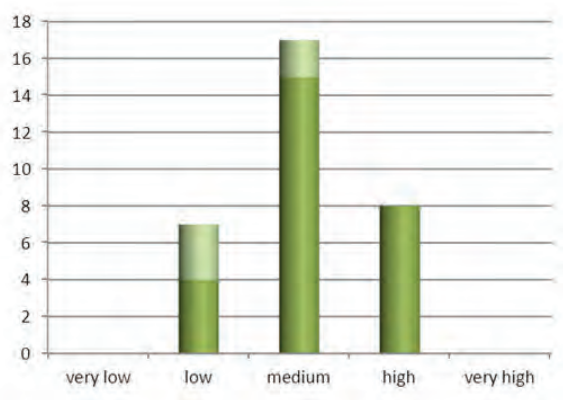

Terrestrial ecosystems

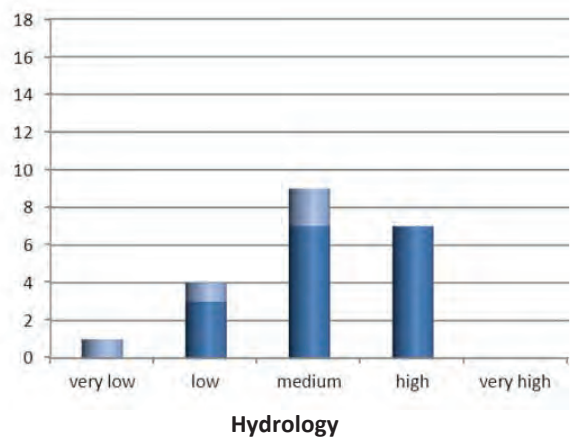

Figure 5.5: Distribution of confidence in attribution of observed impacts to climate change, disaggregated by role (light colours: minor role, dark colours: major role). The vertical axes indicate the number of assessments in the respective confidence bins. Confidence levels are shown across all systems (top left) and then separately for each major impact system.

\subsubsection{Comparison across climate variables}

Atmospheric temperature rise is the most prominent driver of observed change. For more than $60 \%$ of the documented impacts, atmospheric warming is included as one of the drivers of change; this is followed by ocean warming, which is one of the drivers of change in about $23 \%$ of assessments. However, a substantial number of assessments also relate to changes in precipitation (see Figure 5.6); the attribution of impacts to trends in precipitation is, overall, only slightly less confident than 
that for temperature. On average, assessments related to ocean temperature display the highest levels of confidence.

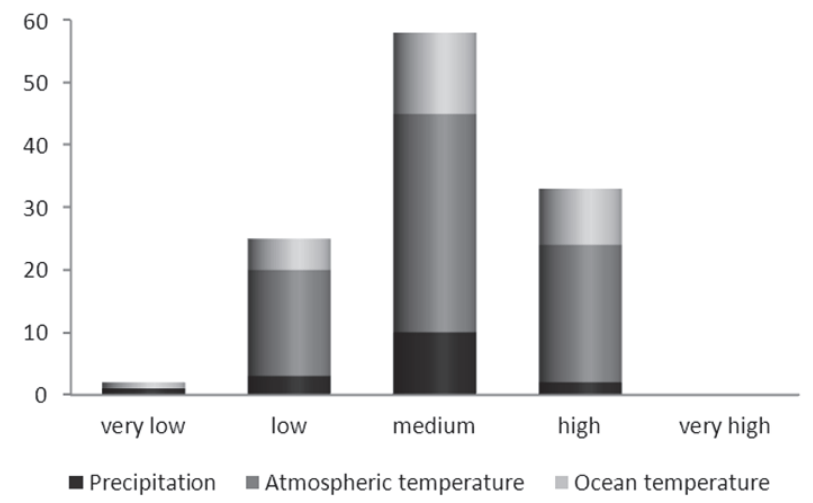

Figure 5.6: Distribution of confidence in impact attribution of the 118 assessments analysed. Vertical axis shows number of assessments in the respective confidence bin, shades of grey indicate corresponding climate driver, i.e. precipitation and atmospheric and ocean temperature.

\subsubsection{Relation to spatial scale}

The distribution of confidence values across region size is relatively even for impact attribution, complimented by a tendency toward relatively higher shares of high confidence assessments in the larger regions and higher shares of low to no confidence assessments in smaller regions (Figure 5.7).

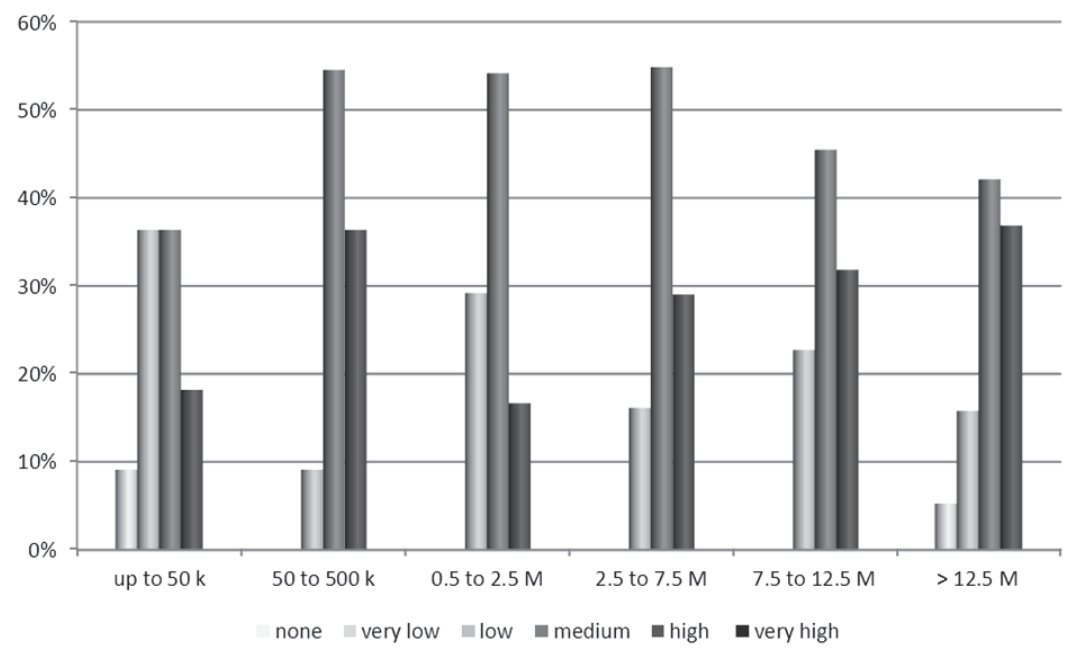

Figure 5.7: Normalized distributions of confidence in impact attribution for a set of six representative region sizes (in square kilometres). Impacted areas are grouped in categories from very small (up to $50,000 \mathrm{~km}^{2}$ ) to extremely large (greater than $\mathbf{1 2 . 5}$ million $\mathbf{~ k m}^{2}$ ). Distribution of confidence levels is normalized to the total number of impacts in that category. Vertical axis depicts the share of total impacts within each category and shades of grey indicate the respective level of confidence. 
Most regions cover between 1 and 10 million square kilometres, corresponding to larger countries or sub-regions (e.g. Scandinavia), extended river systems (e.g. the Amazon basin) and smaller continents such as Europe or Australia. The smallest category comprises impacts at very specific locations, many of them islands. The largest category spans pan-Arctic and pan-Asian impacts, global statements and several extended ocean regions.

\subsubsection{Climate attribution confidence}

In this subsection, the outcome of the confidence algorithm for attributing the observed climate trend to anthropogenic forcing of the climate system is analysed across systems for major and minor roles, climate variables and the role of spatial scale. The analysis differs from that of the impact assessment in three ways. First, the range of confidence values includes the value 'none' for regions with no or very weak observational monitoring, where the observed climate trend is inconsistent with the change required for the impact, or where the observed climate trend is inconsistent with that expected from climate model simulations. Second, the climate attribution step is a systematic analysis without an a priori expectation of the anthropogenic signal. The climate confidence values thus span the entire range from 'none' to 'very high', while the 'very high' label was not once assigned in the corresponding impact assessment, and 'very low' was also rarely used. Third, the algorithm provides confidence assessments for both the attribution of a major role and of at least a minor role (the latter being equal to detection). In the interests of simplification and comparability to the impact assessment provided by Cramer et al (2014) the climate attribution assessment is defined as follows: if the confidence in attribution of a major role is at least medium then that assessment is adopted, otherwise the 'at least a minor role of anthropogenic forcing' (in the following 'minor role' for brevity) assessment is adopted.

Anthropogenic forcing was evaluated as having a major role in 72 out of 118 climate trends ( $61 \%$ of total) and a minor role $(25 \%)$ or no role $(14 \%)$ in the remaining 46 values. For approximately $70 \%$ of the cases, anthropogenic forcing has been detected to have at least a minor role in the observed climate trend with medium confidence or higher. If limited to temperature (both ocean and atmospheric) - thereby eliminating many none or very low confidence assessments attached to precipitation (see 5.3 .2 .2 ) - this value rises to $82 \%$ ( 84 out of 102 ), with $71 \%$ representing a major role (72 out of 102). 


\subsubsection{Major and minor roles and relation to sectors}

In the following, the attribution assessments described in Section 5.3.2 are considered, i.e. whenever the confidence in attributing a major role to anthropogenic forcing yields less than a medium level, the confidence level is shown instead for anthropogenic forcing having at least a minor role.

Despite the substantial number of assessments where observations are not consistent, precluding detection and attribution (14\%), the total confidence distribution is skewed towards high and very high values (see Figure 5.8). More than $50 \%$ of the assessments show higher than medium confidence in attribution to anthropogenic forcing, compared to $29 \%$ lower than medium.

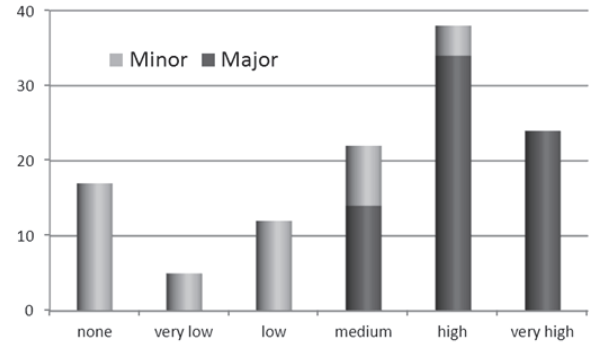

Climate attribution across all impacted systems
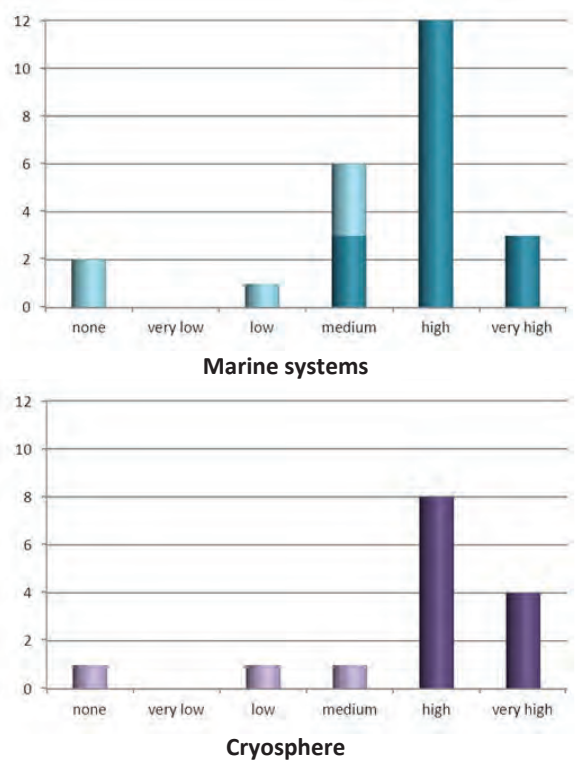

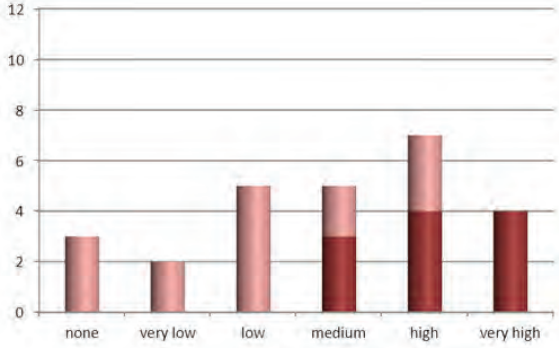

Human and managed systems

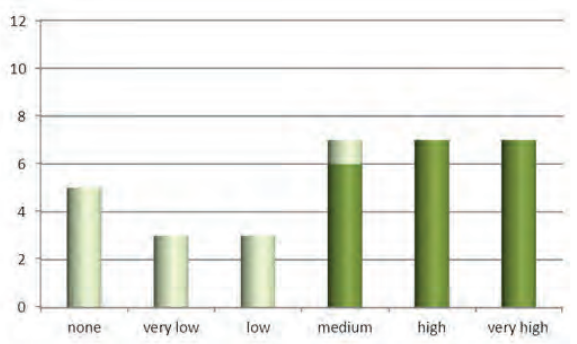

Terrestrial ecosystems

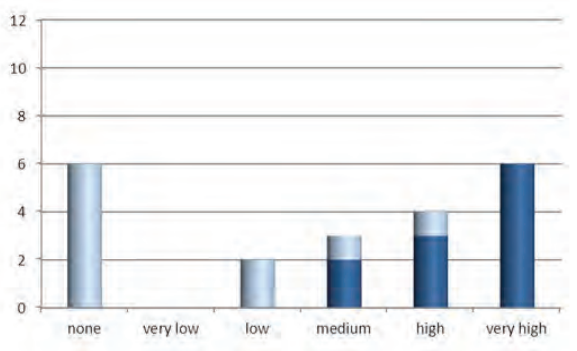

Hydrology

Figure 5.8: Distribution of confidence in climate attribution, disaggregated by role. Light colours indicate a minor role, dark colours indicate a major role of anthropogenic forcing in the observed climate trend. The vertical axes indicate the number of assessments in the respective confidence bins. Confidence levels are shown summarized for all systems (top left) and then separately for climate trends reported to drive impacts within each major impact system. 
The most distinctive feature for the distribution of climate attribution confidence over impact systems is the dominance of high and very high values for climate attribution relevant to the cryosphere and marine systems. The relatively high share of inconsistent assessments relevant to hydrology and, to a lesser extent, terrestrial ecosystems is mainly due to the presence of impacts related to precipitation.

\subsubsection{Comparison across climate variables}

The signature of the climate variable is more distinct for the climate attribution step (where the climate variables are responding) than for the impact attribution step (where the climate variables are drivers of change). More than half of the assessments pertaining to precipitation did not yield a consistent signal across the observational datasets and 14 out of 16 assessments have lower than medium confidence (Figure 5.9). The assessments related to ocean temperature again exhibit relatively high confidence levels. However, in terms of climate attribution, assessments involving atmospheric temperature clearly show the highest confidence levels, with only $16 \%$ indicating less than medium confidence and two thirds presenting high or very high levels of confidence.

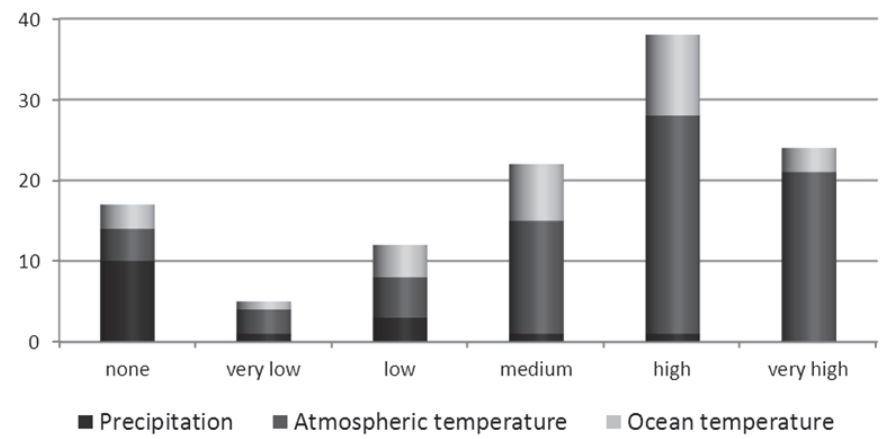

Figure 5.9: Distribution of confidence in climate attribution for the 118 assessments analysed. Vertical axis indicates the number of assessments in the respective confidence bin and shades of grey indicate the corresponding climate variable, i.e. precipitation (16 values total) and atmospheric (74) and ocean (28) temperature.

\subsubsection{Relation to spatial scale}

There is a clear relationship between confidence in climate attribution and the spatial extent of the affected region; this is most prominent for very small and very large regions (see Figure 5.10). Half of the assessments in the smallest regions have no confidence in climate attribution and $90 \%$ of attributions are made with less than a medium level of confidence. Very high confidence in climate attribution is restricted to regions that cover at least 2 million $\mathrm{km}^{2}$, most are 5 million $\mathrm{km}^{2}$ or larger. This is partly due to the algorithm penalizing smaller regions. However, given that the effective 
penalty starts at about 10 million $\mathrm{km}^{2}$, the signal of region size might be expected to be even more pronounced, including for medium-sized regions. While region size is an imposed constraint on confidence in climate attribution, it appears unrelated to other factors that impose more severe constraints: chief among these is the availability of a dense, long-term monitoring network.

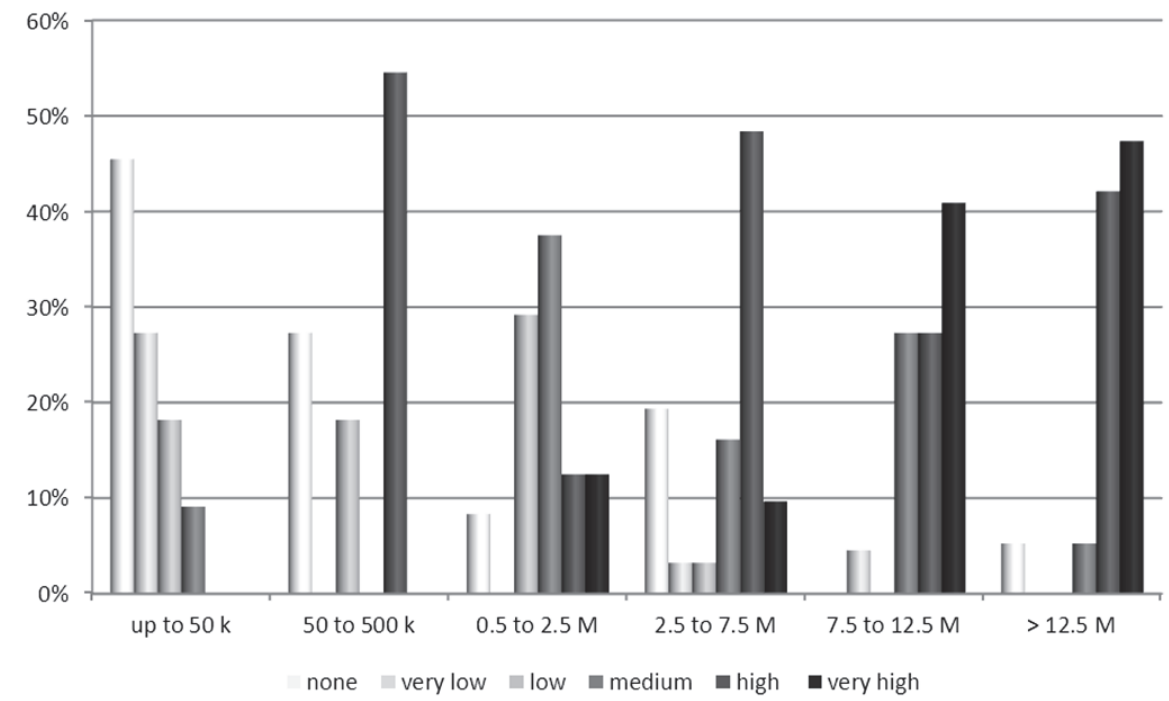

Figure 5.10: Normalized distributions of confidence in climate attribution for a set of six representative region sizes (in square kilometres). Impacted areas that correspond to the climate trends are grouped in categories from very small (up to $50,000 \mathrm{~km}^{2}$ ) to extremely large (greater than $12.5 \mathrm{million}^{\mathrm{km}} \mathbf{2}^{2}$ ). Distribution of confidence levels is normalized to the total number of impacts in that category. Vertical axis depicts the share of total impacts within each category and shades of grey indicate the respective level of confidence.

\subsubsection{Sensitivity to length of period}

To test the sensitivity of our results against changes in the length of the period, the climate attribution confidence algorithm was run again using alternative 30 year periods, $1981-2010$ and 1971-2000 respectively. Overall, the outcome appears robust. A large fraction of the assessments do not change their confidence level, some deviate slightly, with more cases being of lower confidence than of higher confidence for the shorter periods. The effect is more pronounced for the period 1971-2000 than for 1981-2010. The earlier period excludes a decade of pronounced warming (in many world regions), so this matches our expectations. Also, observational data coverage is improved for the later period, due to the increased density of remote sensing networks. The difference between the 40 year period and the 30 year period is small for the detection assessment, but becomes larger for the 'attribution of a major role' assessment. As the major role test is based 
on comparison of the size of the trend against the size of the year-to-year variability, such a result would be expected.

There are very few cases where confidence changes by two or three levels. The regional climate trends for those areas were compared with maps of observed climate trends for 1981-2010 published in IPCC AR5 (Hartmann et al 2013) and it was found that such exceptional decreases occurred in exceptional circumstances, such as local pockets of cooling over parts of North America.

For a selection of impacts that explicitly stated observation periods different from default, the analysis was re-run using the period stated. In parallel, investigations were also carried out on a random sample of those impacts where regional climate trends came out as not related to anthropogenic forcing. For example, Cramer et al (2014) list an increase in soil moisture drought in north-east China due to warming and drying trends for the period 1951-2006. A re-run of the analysis over that period resulted in a slight change in confidence in climate attribution for precipitation from 'none' for the default period to 'very low confidence'. In contrast, climate attribution confidence was lower (medium confidence in a major role) for temperature over the same region for the longer, earlier period than for the default (high confidence in a major role). Another example consists in effects related to a decrease in precipitation in the Sahel region: both the periods 1951-2001 and 1950-2005 were stated in the literature for similar regions related to river flow changes and drought stress on ecosystems. Here, the climate attribution confidence was lifted from 'none' to 'very low' for both periods compared to the default. The Sahel dried from the 1950s through to the 1980s and has then been getting wetter again since the 1990s (Biasutti and Giannini 2006; Greene et al 2009; Mouhamed et al 2013). Consequently, these earlier and longer periods may contain a slight drying signal that is not detectable in the 1971-2010 period.

With few exceptions, deviation from the default period leads to changes in confidence by one degree at most, while the role of anthropogenic forcing stays unchanged. For about half of the tested cases, the outcome of the assessment was not changed at all. Given the cursory nature of this assessment, the default period approach can therefore be considered to be robust.

\subsubsection{Comparison of impact attribution and climate attribution}

The impact attribution assessment is generally less confident than the corresponding climate attribution assessment (see Figure 5.11). The climate attribution assessment also spans a wider range, including a considerable number of 'very high' confidence assessments and, at the other end of the scale, several 'very low' and 'none' evaluations. Because the Cramer et al (2014) assessments of impact attribution have an implicit bias against reporting 'very low' or 'no' confidence cases, the distinction in average confidence is actually understated by these results. 

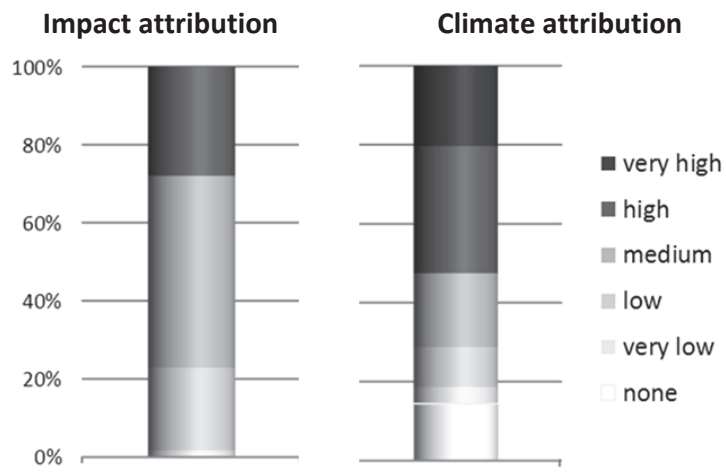

Figure 5.11: Normalized distribution of confidence levels for impact attribution (left panel) and climate attribution (right panel), in percent.

\subsubsection{Direct comparison for individual impacts: attribution}

The following figure shows the distribution of the value pairs for impact and climate attribution for the same impact assessment, irrespective of the magnitude of the roles found (Figure 5.12).

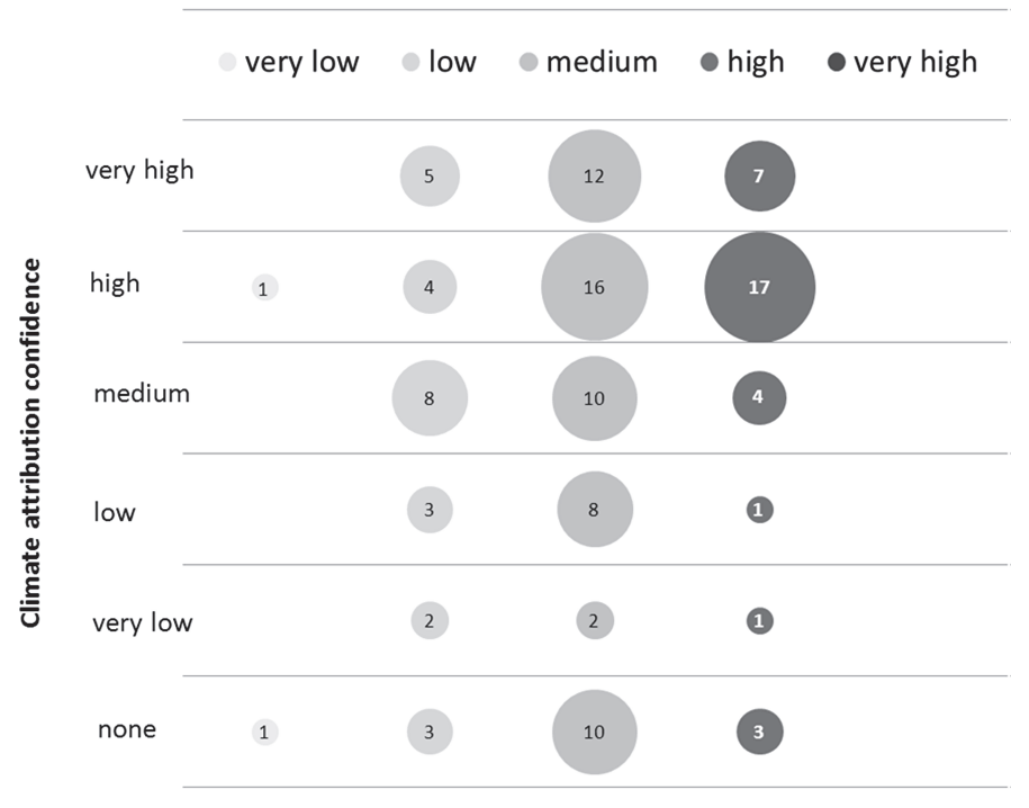

Impact attribution confidence

Figure 5.12: Distribution of confidence levels for impact attribution (horizontal axis) and climate attribution (vertical axis) for the 118 impact-climate trend pairs analysed. Circle area indicates the number of assessments in the respective bin. 
Confidence in climate attribution is clearly considerably higher than confidence in impact attribution. Also, there is obviously no robust direct relationship between confidence values on the impact and climate side. Climate assessments that could not be attributed to anthropogenic forcing at all include the full range of confidence levels on the impact side. Similarly, many climate assessments that show high or very high confidence in a signal of anthropogenic forcing in turn lack confidence in the attribution of corresponding observed impacts to regional climate trends.

While there is a tendency for assessments with high confidence in impact attribution to also be related to anthropogenic forcing with high or very high confidence, this is not exclusively so. The effect is less pronounced for medium confidence impact assessments, which feature a high share of climate attribution assessments with lower-than-medium confidence. For climate attribution, inference about impact attribution confidence is even less justified.

\subsubsection{Double major attribution}

For almost half of the examined assessments (57 values), a 'major role' is assigned for both impact and climate attribution and for 53 assessments, confidence is medium or higher for both steps. The distribution of these double major attribution assessments (hereinafter 'double majors') across systems is shown below (Figure 5.13). Double major impacts are driven exclusively by sea surface $(25 \%)$ and atmospheric (75\%) temperature. The cryosphere and marine systems feature the highest share of double major impacts.

The AR5 highlights impacts that occur on a regional or continental scale, i.e. the impact has been observed for the majority of the potentially affected area of the respective world region (IPCC 2014d). Given the broad nature of the continental assessments and the large spatial extent covered by many of those impacts, a large fraction of these regional scale impacts might be expected to be included in the 'double major' group. Of the continental scale impacts with a major role of climate change in the observed changes, $76 \%$ also have a major role assigned for the climate attribution. Furthermore, for $67 \%$ of the continental-scale cases, the climate attribution assessment yields high or very high confidence for the major role of anthropogenic forcing in the observed climate trend; for only two values, no role of anthropogenic forcing could be found.

However, a substantial number of smaller region assessments (21) are also part of the 'double major' group. The continental assessments are overrepresented though, as they account for just $36 \%$ of the assessments examined in total but make up roughly $60 \%$ of the double major values. While about three-quarters of all continental assessments form part of the double major bin, only about a quarter $(27 \%)$ of the subcontinental assessments shows such strong relations for both the impact and the climate steps. 


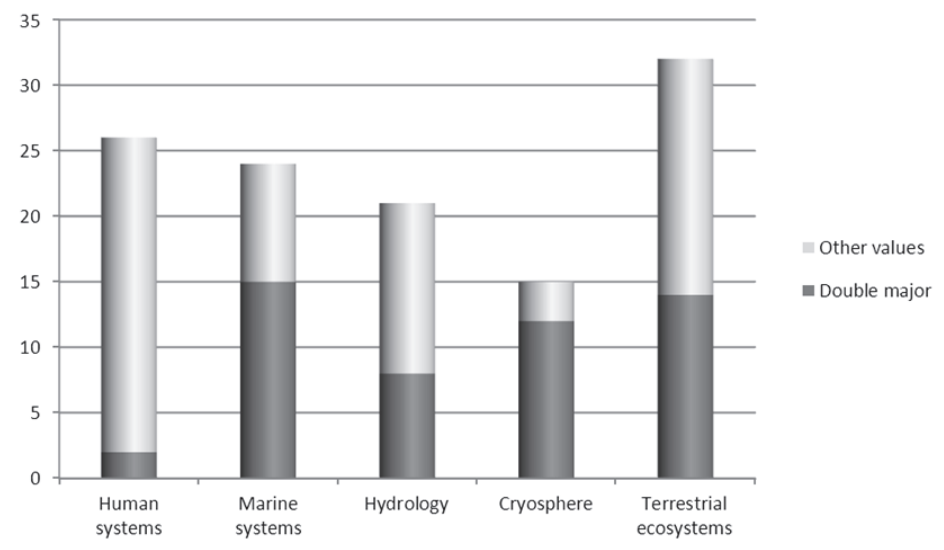

Figure 5.13: Distribution of assessments with both a major role of climate change for the effect observed and a major role of anthropogenic forcing in driving that climate trend across impact systems. Vertical axis shows number of assessments. Double major impacts are exclusively related to warming of the ocean $(25 \%)$ and the atmosphere $(75 \%)$.

\subsubsection{Direct comparison for individual impacts: detection}

In order to complement the assessment of the impact and climate attribution confidence across the wide range of impacts covered in Cramer et al (2014), Figure 5.14 shows a comparison of confidence between impact and climate detection. Detection refers to the documentation of a non-zero effect of the respective driver in observations. As expected, confidence levels are shifted to higher levels for both steps. For impact detection, only ten assessments have less than medium confidence (compared to 27 for attribution) and a few very high confidence assessments prevail. The shift on the climate side is even more pronounced, with the number of very high confidence assessments more than doubled compared to the attribution assessment, accompanied by considerably lower numbers of low and medium confidence assessments. As a result, the share of values where impact and climate detection confidence occupy opposite extremes is even further reduced. However, the fact remains that there is no robust direct relationship between a high confidence assessment for the impact step and one for the climate step that would allow an inference of confidence in detection of anthropogenic influence in observed impacts from one of the two steps alone. 


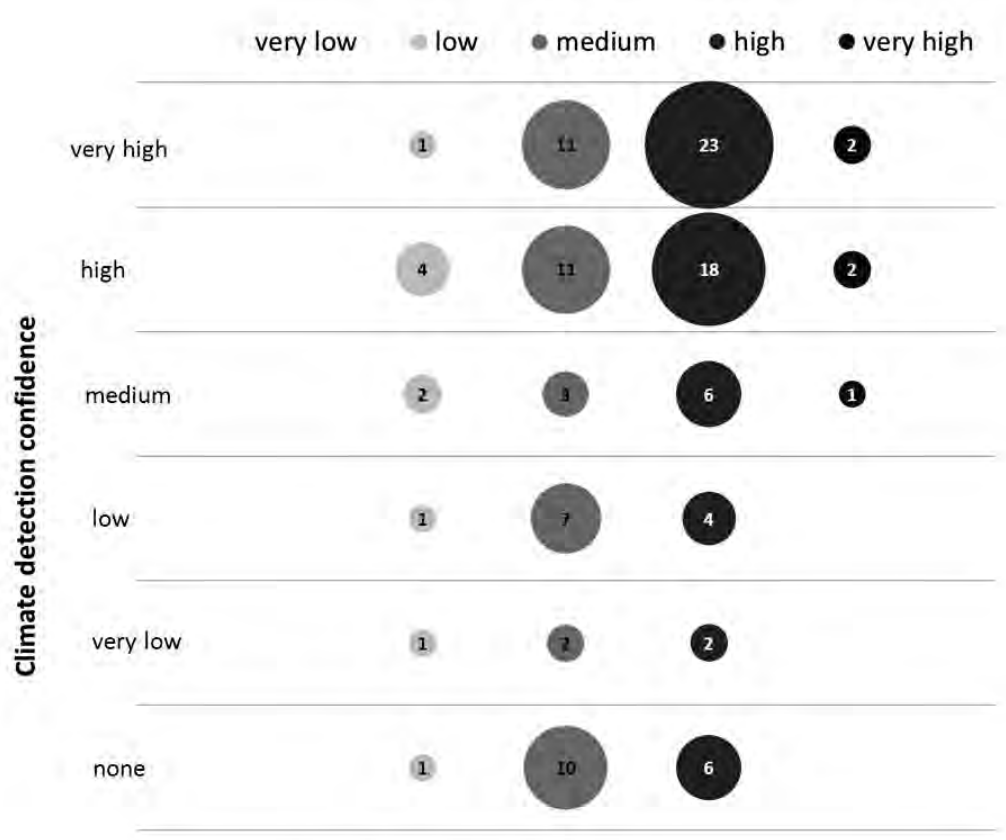

Impact detection confidence

Figure 5.14: Confidence in detection of a climate change impact (impact detection, horizontal axis), and in detection of an anthropogenic signal in the corresponding climate trend (climate detection, vertical axis) for the set of impact-climate trend pairs described in Section 5.2.1. Circle areas indicate number of assessments in the respective bin.

\subsubsection{Combined assessment}

A simple minimum value approach was used to assign both role and confidence to the combined assessment. In cases where two climate variables had to be assessed for the same impact, assessments were handled separately. As many of these are combinations of precipitation and temperature, confidence in the climate attribution tends to differ considerably between the two climate variables. Figure 5.15 shows the distribution of the resulting combined confidence and the distribution of impact and climate attribution confidence levels for comparison. As previously discussed, almost half of the assessments feature a major role for both drivers analysed here with at least medium confidence. As a result of this, a substantial part of the assessment remains confident in a major role of anthropogenic climate change for the impacts observed in the combined, although both the distribution of confidence levels and the number of major role assessments are reduced considerably. A review of the underlying tables reveals that most of the statements with high confidence in the attribution of the observed impact to anthropogenic climate change relate to the 
cryosphere and ecosystem impacts from the high northern latitudes and the degradation of tropical coral reef systems (see Annex B). Impacts on the livelihoods of Arctic indigenous peoples are the only incidences where this analysis confirms a major role for anthropogenic climate change for effects observed within human and managed systems. Combined attribution confidence is medium for nine of twenty-three temperature-related impacts observed in human systems, albeit the role is minor for seven out of those nine cases.
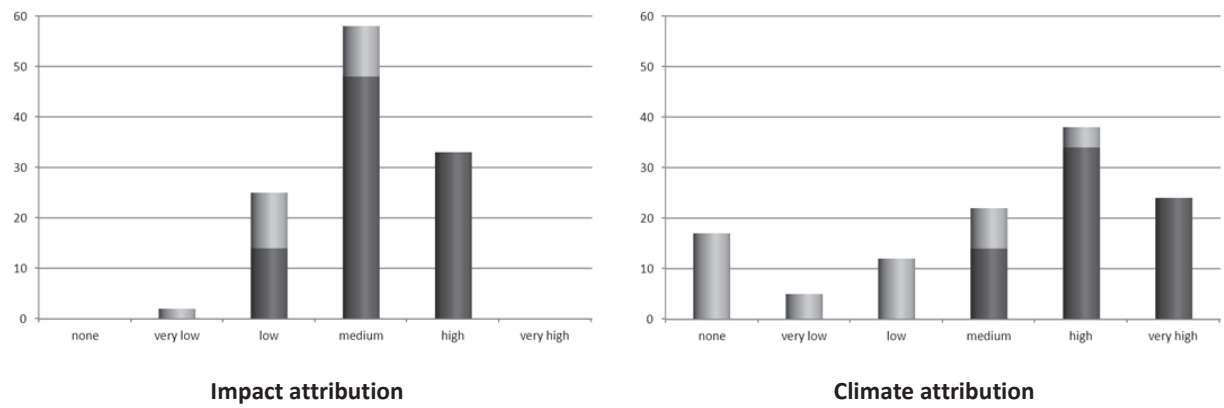

60

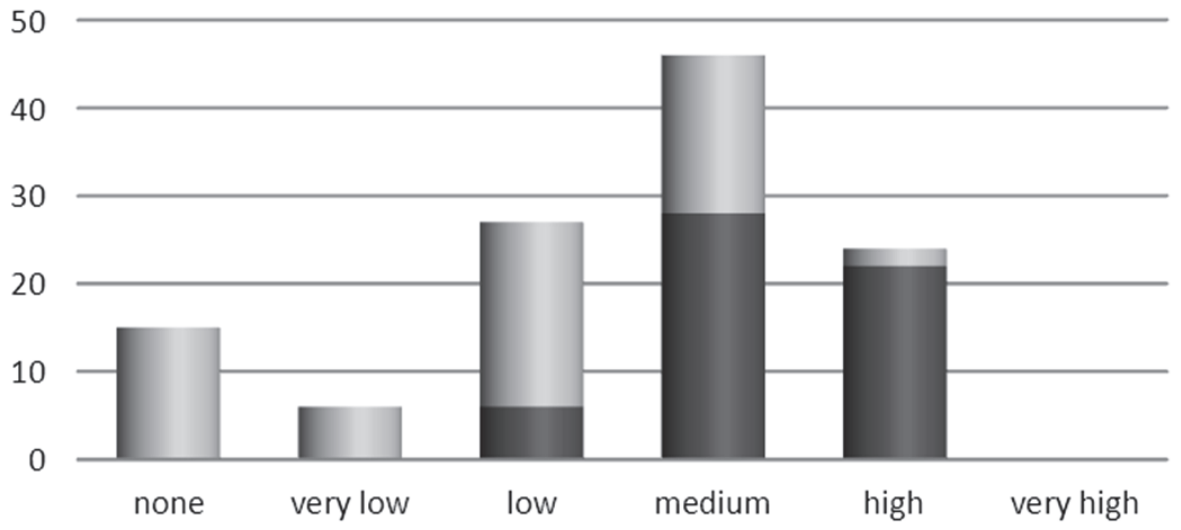

Major role $\square$ Minor role

Combined attribution (minimum approach)

Figure 5.15: Assessment of the confidence in the role of anthropogenic forcing for observed impacts of climate change using a multi-step approach, with application of the 'minimum confidence' method for combined attribution (bottom panel). Assessments of confidence in the attribution of observed impacts to climate change (upper left) and confidence in attribution of changes in climate to anthropogenic forcing (upper right) for the climate changes contributing to the impacts observed are shown for comparison. All assessments differentiate between a minor (light colour) and a major (dark colour) role of the respective driver in the observations, compared to that of other drivers of change.

Overall, 66 of the 118 impact statements are attributed to anthropogenic forcing of the climate system with at least medium confidence $(56 \%)$, and none of these is mediated by precipitation. For 
detection, the number of combined assessments with at least medium confidence rises to 77 (65\%). If limited to effects of warming, approximately $65 \%$ of the assessments are attributed to anthropogenic forcing with at least medium confidence; this number rises to almost three-quarters if detection is considered instead of attribution.

Another important factor for the attribution of impacts to anthropogenic forcing is the spatial extent of the affected area. As can be seen in Figure 5.16 , approximately $75 \%$ of the impacts that occur over very large areas of more than 7.5 million square kilometres are attributed to anthropogenic forcing with medium or high confidence. This ratio is considerably lower for the small and mediumsized regions, with medium or high confidence represented in $56 \%$ and $22 \%$ of the cases respectively. In contrast, approximately half of the impacts occurring in smaller regions have no or very low confidence attached to the combined assessment.
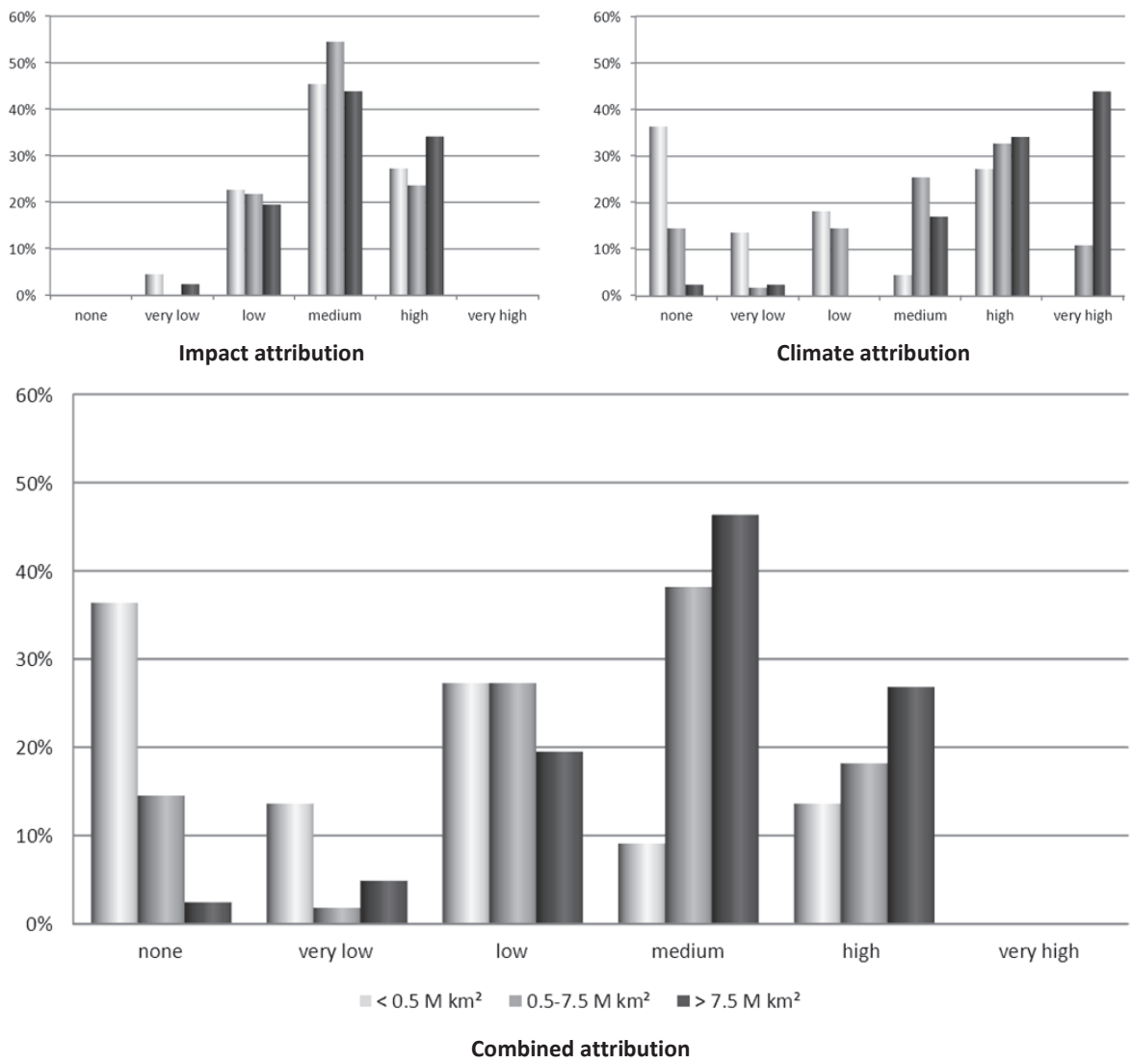

Figure 5.16: Normalized distribution of attribution confidence over region sizes, for combined attribution confidence (bottom panel), and impact attribution (top left) and climate attribution (top right) assessments for comparison. Normalization is to all assessments within the same regional category. Regions are grouped into 3 categories, up to half a million square $\mathrm{km}$ (left bar), 0.5 to $\mathbf{7 . 5}$ million square kilometres (centre bar), and larger than 7.5 square kilometres (right bar). 


\subsection{Discussion}

This analysis provides the missing link between the observed effects of recent climate change reported in AR5 and the role of anthropogenic forcing of the climate system for the climate trends related to these observations. While the methodological approach falls short of a full-scale end-toend analysis of individual impacts, it provides a robust first assessment of the role of anthropogenic climate change in a wide range of climate-related effects that have been observed around the world.

Results show that, on average, levels of confidence for attributing the observed climate trends to anthropogenic forcing are higher than levels of confidence for attributing observed effects to climate trends. Given that, by design, the initial impact database looks at areas where impacts would be expected, excludes negatives and reports few statements of very low confidence, climate attribution might have been expected to be less confident compared to impact attribution, rather than more. On the climate side, a systematic analysis is performed without an a priori expectation of the actual anthropogenic signal. The fact that confidence on the impact side is the limiting factor for many assessments indicates a need for more research into the effects of regional climate trends, in particular with regard to their interaction with other factors.

Climate attribution confidence is notably higher than impact attribution confidence for effects reported as a consequence of atmospheric temperature change and, to a slightly lesser degree, of ocean temperature change. This result suggests that for impacts mediated by temperature change it is feasible to extend the rigorous impact detection and attribution analyses available for the effects of regional climate trends to the effect of anthropogenic climate change and thus fill the gap noted in the AR5.

In contrast, the confidence in impact attribution is generally higher than the confidence in climate attribution for effects related to precipitation. The majority of assessments related to precipitation fail the first test for consistency between climate trends reported in AR5 and those found in the global observational data products used. This points to the limited ability of GCMs to reproduce spatial and temporal precipitation patterns correctly, which is largely due to the fact that precipitation is highly variable and the direct response to anthropogenic forcing relatively weak. However, another cautious interpretation is that the sensitivity to short and medium-term fluctuations in precipitation is not necessarily a good indicator for exposure to long-term anthropogenic climate change.

Generally, combined attribution confidence increases with size of affected area, mostly due to the characteristics of the climate attribution step, as positive correlation with size is much less pronounced for impact attribution. Very high confidence in climate attribution is found exclusively 
for areas larger than 2 million square kilometres. While larger spatial scales often reduce uncertainty, confident climate attribution is also possible on subcontinental and smaller scales. Interestingly, a substantial number of double major effects were associated with relatively small regions. This is surprising as warming trends due to anthropogenic forcing are generally more evident in global or continental mean records, where most of the local variability is averaged out, than in time series of local temperature.

This analysis clearly links a large majority of the continental scale, major role impact attribution assessments highlighted in the AR5 to anthropogenic forcing. For about three-quarters of these assessments, the driving climate trend can be attributed to anthropogenic influence with at least medium confidence for a major role; two-thirds even yield high or very high confidence ratings. All of these assessments are driven by warming. Confidence in detection of at least a minor role of anthropogenic forcing in the climate trend across all impact assessments analysed is at least medium for a similar share of values (65\%). This figure rises to about $75 \%$ when only evaluating impacts related to temperature.

The cryosphere and marine systems emerge as hotspots of impacts of anthropogenic forcing in the combined analysis. This is to be expected for the cryosphere, given that it has long been identified as a key sensitive region and that it is often situated in areas of amplified warming, such as mountains or the Arctic region. Indeed, the measurement of glacial retreat is often interpreted as a proxy measurement of global temperature trends (Vaughan et al 2013). The high representation of marine impacts may in part be due to selection bias. Though the oceans cover $70 \%$ of the planet, fewer statements related to marine ecosystems are available than for terrestrial ones, suggesting that only those impacts have been included in the database that show a very clear relationship to climate change. Other possible explanations include the strong influence of ocean currents and long-term natural climate variations on ocean temperature. As a consequence, the climate change related signal-to-noise ratio for oceanic warming is often lower than for terrestrial warming. The resulting data requirement to detect any effect is high and may therefore be sufficient for high confidence in attribution following the approach taken here. Finally, despite the confounding effect of harvesting, marine species are freer to respond to thermal change in detectable ways than many species living in fragmented terrestrial habitats.

Impacts on the livelihoods of Arctic indigenous peoples are the only incidences where this analysis confirms a major role for anthropogenic climate change for effects observed within human and managed systems. However, combined attribution confidence is medium for nine of twenty-three temperature-related impacts observed in human systems, albeit the role attribution is minor for seven out of those nine cases. The lower confidence in combined attribution for human systems is 
mainly due to the comparatively minor role of recent climate change in most impacts observed in human and managed systems and the limited confidence in some of those observations.

All in all, our analysis confirms earlier statements that anthropogenic climate change is causing discernible impacts on natural systems worldwide. It also extends the analysis to include some human and managed systems. The lack of a robust relationship between impact and climate attribution shown in Section 5.3.3 makes it clear that the inference of end-to-end attribution from only one of these assessments is inappropriate.

Both assessments and their combination have some shortcomings, which will be briefly discussed below. The initial list of impacts by Cramer et al (2014) that served as an input to this analysis is based on purely qualitative assessment derived from expert elicitation and from peer review to calibrate against the common guidelines and between the different assessments. This approach has pioneered outreach and allowed for the inclusion of impact categories that were not formally assessed in earlier reports. However, it shares the common weaknesses of expert elicitation, such as a lack of transparency and a higher degree of subjectivity compared to quantitative assessments. Another effect is the tendency of expert panel results to veer to the middle - in the absence of clearly reproducible criteria and hard, quantitative standards, elicitations tend to avoid extreme values and stay within the 'safe' middle ground, especially under uncertainty. The assessment may therefore not represent the full range of uncertainty on the impact side. Some ambiguity also exists regarding the definition of 'major role' and what distinguishes 'attribution of at least a minor role' from detection. The aggregation level of the analysis in Cramer et al (2014) varies considerably between individual assessments. In some cases, the resulting meta-level assessment seems overly simplified, and may not adequately represent the complex relationships of the individual and combined climate factors and their causal roles for the impacts observed.

The climate attribution step is based on the output of (global) general circulation models and global gridded sets of observational data. Regional observational products and climate model data may be more accurate (at least for most regions). Both fundamental and technical aspects of the construction and implementation of global climate models limit their ability to accurately represent the climate system at regional and local scales. Downscaling techniques could assist the use of global climate models, but global downscaled simulation data applicable to attribution analysis is still not available. The algorithm recognizes this limitation in several steps, for instance in tuning down confidence for smaller regions. Similarly, the global observational data sets were mainly chosen for consistency. 
The confidence algorithm applied for climate attribution is essentially a quantitative approach that is then transferred to a qualitative scale. While there is a chance that a systematic bias has been introduced despite calibration, the operation is still based on the same routine for all assessments. It therefore provides a higher degree of standardization compared to the impact side of the assessment. By considering uncertainty in observational monitoring, the attribution algorithm goes beyond most contemporary climate change detection and attribution studies (see Bindoff et al (2013) for examples). Still, both the choice of weighting factors and the way the numerical factors are converted to a qualitative scale are based on lose constraints and could allow the development of alternative approaches that may yield different results. The steps that tend to dominate in determining the final confidence assessment are those where the constraints on the interpretation are quite tight, for instance with respect to the existence and density of observational monitoring (see Annex A).

The use of the simple minimum value approach to derive estimates of confidence in combined attribution is arbitrary to a certain degree, although it is procedurally straightforward. A common feature of all measures of combination is the loss of information about the source of uncertainty and a loss of information specific to the individual steps. While many consider attribution to anthropogenic forcing the main goal of detection and attribution exercises (Hoegh-Guldberg et al 2011; Hegerl and Zwiers 2011), the necessary degree of information aggregation may lead to a loss of information that is very relevant in the context of vulnerability and adaptation.

Similarly, this analysis inherits its limitation to the effects of long-term climate trends from the setup of the AR5 assessment. The design probably excludes certain impacts, for example step changes or impacts of changes in temporal or spatial patterns of climate that are not easily detected in trends of aggregate climate variables. The analysis currently excludes the assessment of impacts related to sea level rise. It is also not well-designed for capturing effects of changes in variability, which is a major drawback given the vulnerability of systems and societies to extreme weather. While not decisive for the purpose of this chapter, i.e. providing a synthetic assessment of the role of anthropogenic forcing in observed impacts as reported by IPCC's AR5, assessing the role of anthropogenic forcing for changes in variability is nevertheless crucial for the development of rapid climate attribution assessments as a climate service.

The design is sensitive to the length of the observation period and its start and end year. Though the sensitivity tests in 5.3.2.4 show relatively robust results for the climate attribution analysis, it was not possible to do a similar analysis for the impact statements; a combination of deviations in confidence for both steps will influence the overall outcome more strongly. 
To enhance the usefulness of a detection and attribution analysis for decision makers, it is crucial to improve the reproduction of precipitation patterns and changes in variability. Still, the primary condition to improve knowledge about past effects of anthropogenic climate change that emerges from this analysis is to reduce the uncertainty about the role of changes in climate on the impact side.

\subsection{Conclusion and outlook}

The analysis provided in this chapter confirms earlier statements that overall, anthropogenic climate change exerts a discernible effect on natural systems worldwide. This statement can even be extended to include some human and managed systems. However, the combined attribution analysis for the individual impacts makes it very clear that no inference can be declared between high confidence in climate attribution and confidence in impact attribution, or vice versa. Therefore, unless other factors are taken into account, drawing conclusions about the role of anthropogenic forcing in climate-related impacts using only one of the two attribution steps alone means extrapolating blindly.

The signature of anthropogenic influence is still not detectable for most changes in precipitation. Improvements in the reproduction of precipitation patterns and the representation of climate model results at smaller spatial scales are essential, especially for the attribution of impacts in tropical regions. For temperature-related changes, the uncertainties surrounding the attribution of impacts to climate change prevail over those related to the attribution of climate change to human influence. More in depth analyses of the role of climate compared to other factors in observed changes in natural and human systems are crucial in order to improve understanding of the role of anthropogenic forcing in climate-related impact. 
6 Global distribution of observed climate change impacts

The scarcity of robust scientific evidence supporting the attribution of observed impacts to climate change in some vulnerable regions does not indicate that no such impacts have occurred.

Publication corresponding to this chapter:

Gerrit Hansen and Wolfgang Cramer (2015) Global distribution of observed climate change impacts. Nature Climate Change 5:182-185 [Commentary] 
The scarcity of robust scientific evidence supporting the attribution of observed impacts to climate change in some vulnerable regions does not indicate that no such impacts have occurred.

Impacts of recent climate change have now been documented in all major regions of the world and in many natural and human systems. This is one key conclusion of the IPCC's Fifth Assessment Report (AR5), which was recently completed with the approval of its Synthesis Report in Copenhagen (IPCC 2014e). Over the last two decades, coverage of such observed impacts has been expanded from an initial focus on land ecosystems towards the marine realm, and to important features of human and managed systems such as food production and human health. A central graphic from the IPCC working group II (WGII) 'Impacts, Adaptation and Vulnerability', a world map with impact icons symbolizing localized or regional-scale attributed impacts, was received with enthusiasm by the press and social media (Figure 6.1). The array of impacts of recent climate change that have been observed globally is impressive. However, it is important to recognize that this map represents the state of knowledge on impacts that have been attributed to climate change, compiled through a rigorous analysis of the scientific literature. It is not a comprehensive summary of all adverse effects that could plausibly be linked to climate change.

\section{Documented impacts}

The evidence base for climate change impacts, from monitoring systems and environmental research, has been growing during the last two decades (Cramer et al 2014). However, there still are more and higher quality observations for such impacts in mid to high northern latitude regions than elsewhere. Some readers wrongly perceived the map (Figure 6.1) as indicating that the northern regions have been more strongly affected than southern regions. Also, the comparatively sparse documentation of climate change impacts in some of the most vulnerable regions of the globe could create the impression that the global burden of climate change impacts was not adequately portrayed. Hence a risk of misinterpretation arising from the uneven distribution and poorly defined spatial resolution of the icons on the map was identified during the finalization of the Synthesis Report.

To address these concerns, the philosophy for the map of the WGII findings was refined for the IPCC Synthesis Report. The revised map was produced by removing the spatial information about geographic location at the subcontinental scale. Instead, comprehensive lists of impacts identified for each world region were presented. In addition, a quantitative indication of the uneven regional distribution of climate change literature was provided (Figure 6.2). This change aligned the data in the figure with the data in the underlying table, increased emphasis on the global distribution of observed impacts, and highlighted the issue of uneven literature coverage. While both figures 
contain the same data, the difficulty of their appropriate representation in a policy context highlights the need for better communication of the scientific basis for impact assessment, including the need to explain the full potentials and limits of detection and attribution analysis.

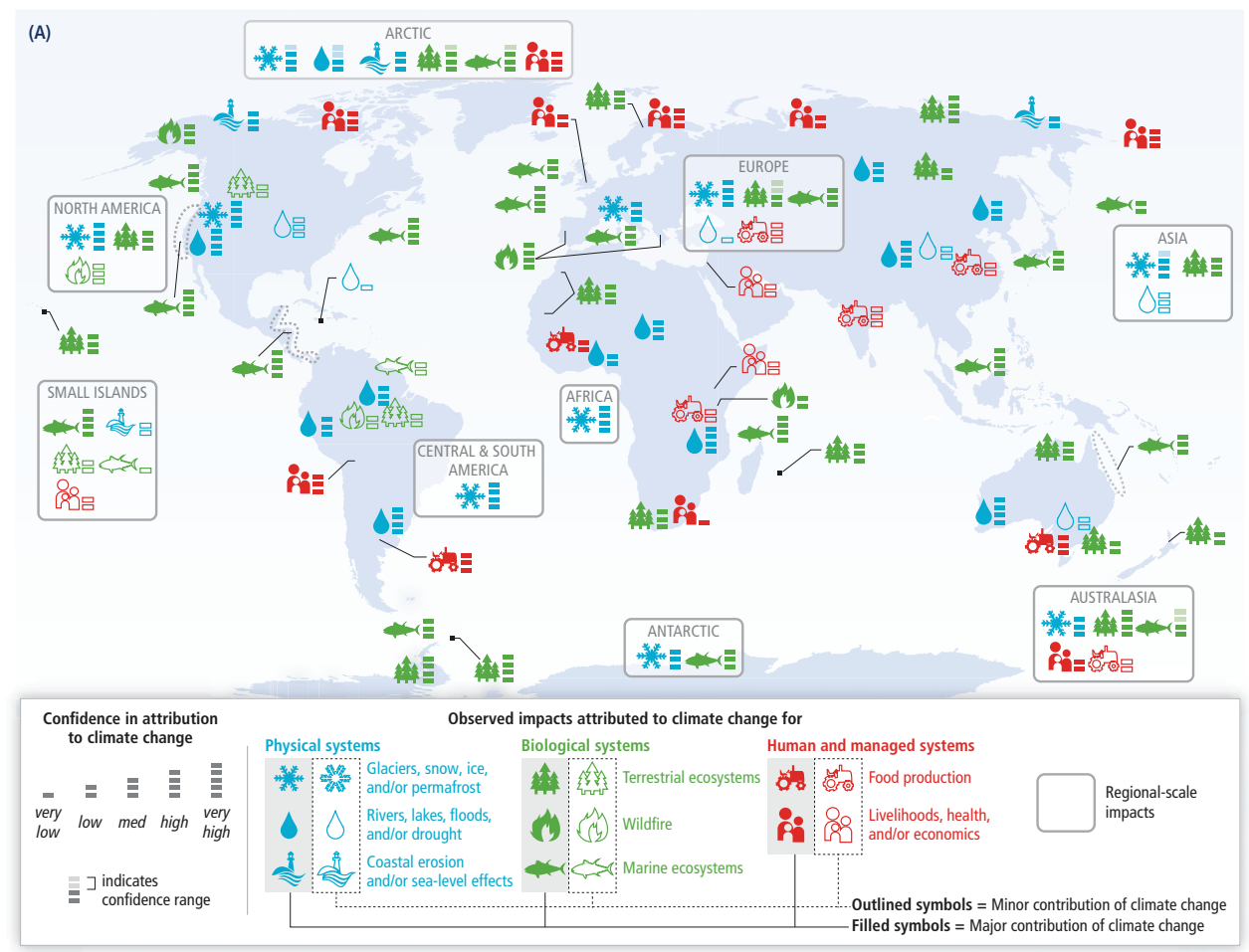

Figure 6.1: Observed impacts of climate change. Global patterns of impacts in recent decades attributed to climate change, based on studies since the Fourth Assessment Report. Impacts are shown at a range of geographic scales. Symbols indicate categories of attributed impacts, the relative contribution of climate change (major or minor) to the observed impact, and confidence in attribution. Source: Figure SPM2.A in IPCC (2014d).

\section{Standards}

Scientific attribution of observed impacts to climate change requires time series of observations of sufficient length and quality for the affected system, and for both climatic factors and other important drivers of change, such as land use or economic development. Mere correlation between the changing climate and its presumed impacts is insufficient for attribution. Instead, understanding of all likely causes of change and their interaction is needed (Hegerl et al 2010; Stone et al 2013). For a specific impact to be included in an IPCC assessment, a diligent examination of that specific case in the peer reviewed literature must be available. As a result of these requirements, well-studied regions and systems with few confounding factors combined with a high sensitivity to climate, 
feature more prominently in the list of attributed impacts (Rosenzweig and Neofotis 2013; Cramer et al 2014). The focus of attribution assessments is different from that of vulnerability or impact studies that assess how impacts of future climate change will unfold, based on the sensitivity of a system to climatic factors, expected future climate change, and socio-economic factors delineating vulnerability. For such studies, the sensitivity of a system to climate change is often inferred from past responses to climate variability. However, impacts of natural climate variability do not constitute impacts of climate change.

Widespread impacts attributed to climate change based on the available scientific literature since the AR4

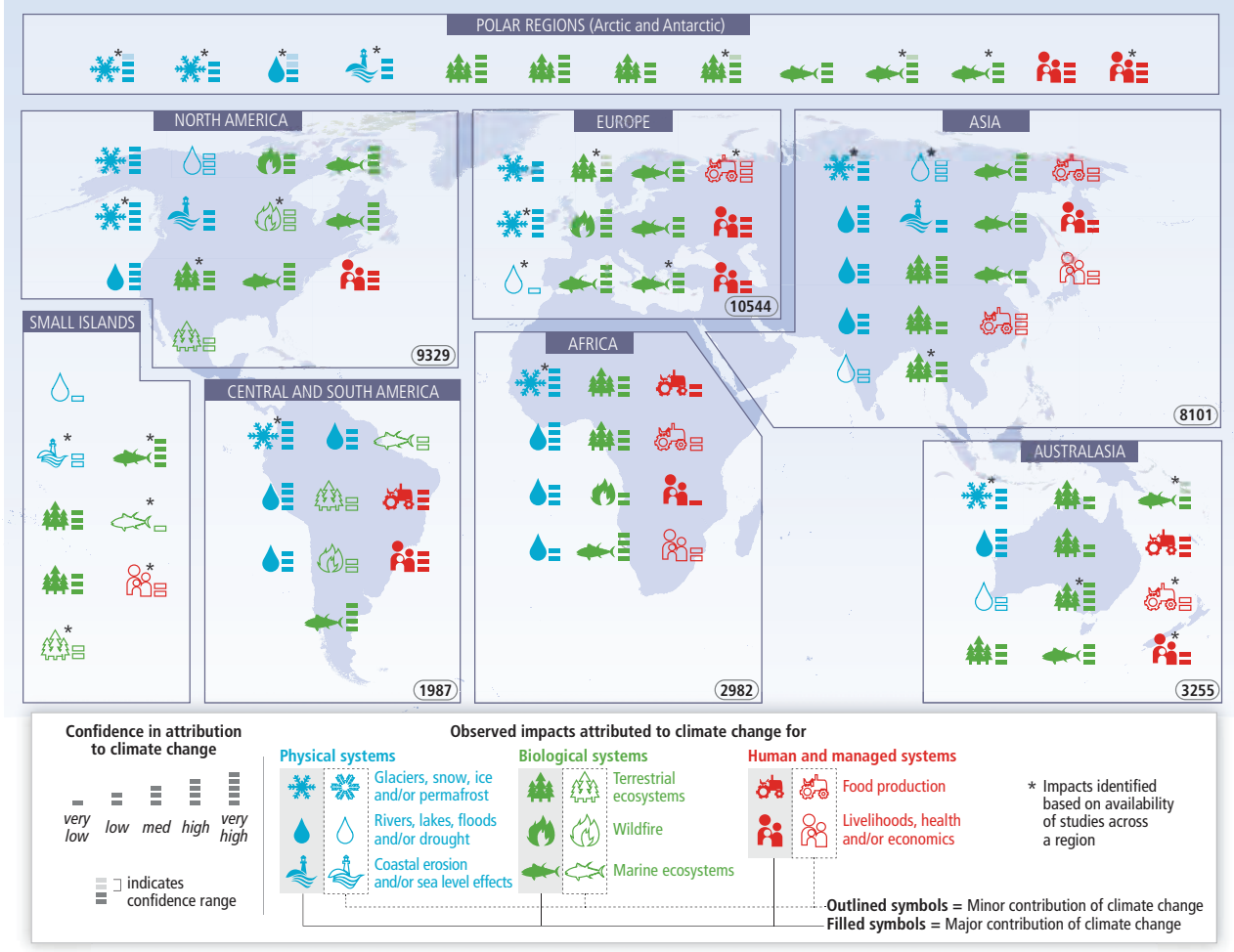

Figure 6.2: Revised schematic of the observed impacts of climate change. Symbols indicate categories of attributed impacts, the relative contribution of climate change (major or minor) to the observed impact, and confidence in attribution. The numbers in ovals summarize the number of all climate change related scientific studies published between $\mathbf{2 0 0 0}$ and $\mathbf{2 0 1 0}$ for each region, as a proxy for the difference in the regional literature base. Source: Figure SPM.4 in IPCC (2014e).

\section{Vulnerability}

Responses to climate variability, for example harvest failure due to drought, are often more easily detected than responses to gradual changes in climate. However, a long-term change in climate variability - which would constitute climate change - is difficult to detect. Observed trends in 
frequency or intensity of climate extremes are still less conclusive, though trends have been documented for some types of extremes, in particular heat waves and heavy precipitation, in many regions (Seneviratne et al 2012; Peterson et al 2013a). Also, areas influenced by long-term natural climate modes such as the El Niño Southern Oscillation face an additional challenge in detecting a persistent trend in climate against the baseline of periodic change, and therefore in attributing observed effects to climate change.

In turn, this means that some of the most pronounced adverse effects related to climate, i.e. those caused by extreme weather, can presently not be attributed to climate change directly even though they might be consistent with what one would expect to happen under a changing climate (James et al 2014). Progress is being made in assessing the role of anthropogenic forcing in occurrence and intensity of extreme weather (Coumou et al 2013) and individual events (Peterson et al 2013b; Herring et al 2014), but information on the latter is only available for a small set of events.

\section{Human systems}

A large fraction of the most robust evidence for impacts of recent climate change is related to the cryosphere, or to ecosystems that are sensitive to temperature. In contrast, evidence for human systems is relatively sparse. Much of the reason for this is that humans are remarkably adaptable and often make adjustments in response to risks or impacts. In addition, humans operate in a complex world, with many factors changing simultaneously. Human systems can be impacted by climate change either directly, for example in the case of increased heat-related mortality due to more frequent heat waves, or indirectly, by cascading effects of changes in the natural environment triggered by climate change. As the impacts of climate change become more pervasive in the natural environment, impacts on human systems that depend on them would be expected to unfold. Such impacts have been reported by many indigenous communities in high Northern latitudes (ACIA 2005; Ford and Pearce 2010; Arctic Council 2013). However, given the multitude and strength of other drivers of change, combined with the difficulty to assess services delivered by ecosystems, such cascading impacts on humans due to regional changes in climate are not yet well documented in other environments.

Another reason for the weaker documentation of some human systems impacts is the difference in disciplinary approaches to establish causality between quantitative and qualitative sciences (Stone et al 2013). Detection and attribution standards have been developed by natural scientists, and usually rely on statistical methods and numerical models (Hegerl et al 2010). Some areas of explicit concern in the context of climate change, such as impacts on small-scale farming, informal economies and settlements, livelihoods and poverty are predominantly discussed in literature that is 
qualitative in nature, and do not easily lend themselves to statistical approaches. Indeed, a large part of that literature is focussed more on current vulnerabilities and future risks in a context of multiple stressors rather than quantitative evidence of already-manifested impacts.

\section{No evidence of absence}

The recent IPCC report has shown that it is possible to integrate different sources of evidence, data of differing quality and disciplinary approaches into an overarching assessment of impacts attributable to climate change (Cramer et al 2014). The assessment combines numerous published studies based on observational records of observed change, as well as on documented change in one or several climate variable(s). In some cases, despite the expectation that impacts might have occurred, individual elements of the causal chain leading from changes in climate to changes in the respective impact system were not addressed in scientific studies, making attribution impossible. Coastal degradation is a prime example of the difficulties met when assessing observed impacts: tide-gauge records documenting local sea-level rise may not be available or incomplete for some regions. Even if those records were available, the observed changes in sea level may have causes other than global warming: sediments trapped by large dams, changes in local current systems and subsidence due to hydrocarbon or groundwater removal all contribute to relative sea-level changes (Nicholls et al 2009; Syvitski et al 2009). In addition, impacts of higher sea levels depend on flood remediation, changes in coastlines due to infrastructure and urban developments, settlement patterns and other factors determining risk from floods. So while it is likely that, in many coastal settings, recent damages are partially caused by global sea-level rise, the absence of sufficient data often precludes attributing that impact to any particular cause.

Hence, while the presence of an impact icon on the map is always based on detection of a specific change and its attribution to climate change, the absence of an icon can be due to a wide range of reasons. These include the lack of studies addressing a certain impact, or the failure of the available studies to rigorously attribute an observed change to recent climate change, but none of these can be taken to imply that no such impacts have occurred.

\section{Attribution and risk}

The scientifically robust attribution of observed impacts to climate change is important for several reasons, including overall system understanding and the development of resilient strategies for adaptation, as it examines important drivers of change and their interaction. But a summary of attributed impacts is not a complete inventory of current effects of climate change, or sole indicator of present and future risk. The manifestation and attribution of a certain climate change effect 
obviously carries a strong message concerning future risk. However, the fact that an impact has not occurred, or has not been documented, offers no indication of the absence of such a risk.

At the same time, although climate change may act synergistically with other risk factors and will continue to gain importance as the rate and scale of climate change increases, it must be recognised that the most important driver of current risk for human systems related to environmental degradation is not necessarily (global) climate change, but also other issues such as land-use change, air pollution and poverty.

\section{Unambiguous message}

The map originally provided by WGII (Figure 6.1) informed about the status of knowledge on observed and attributed effects of climate change with some regional specificity. In one sense, empty spaces and missing icons provide information about the current gaps in that knowledge. However, many factors could contribute to these gaps, including the possible lack of data, a shortage of scientific studies or the actual absence of any impacts of climate change.

Both representations are valid ways to convey a large amount of complex information in a scientifically consistent way. However, what the scientific community perceives as useful extra information could be confusing or misleading to another group of stakeholders. The revised version of the map (Figure 6.2), while losing some of the spatially explicit information, addresses important sources of concern and highlights the main message of the assessment: the fact that impacts of climate change occur worldwide and the urgency of addressing climate change. 


\section{Synthesis}

This thesis is rooted in the analysis and expert elicitation performed in the context of the IPCC's working group II Fifth Assessment Report (hereinafter WGII and AR5 respectively). It carries forward questions that remained open after the completion of the fifth assessment cycle and narrows gaps left by the lack of integration between the WGI and WGII assessments. During the AR5 cycle, the widespread perception that anthropogenic climate change is already affecting a number of vulnerable human and managed systems was contrasted by the relative lack of documented evidence of observed impacts of climate change for those vulnerable systems. This inconsistency was exacerbated by the failure of AR5 to assess the link between the impacts caused by regional climate trends and the contribution of anthropogenic forcing to these climate trends. To address these issues was a major motivation for this thesis.

During the AR5 process, gaps in knowledge and understanding about the concept and requirements of impact detection and attribution apparently caused irritation within the extended scientific author and reviewer team. These controversies and/or misperceptions were repeated in a similar form during government review and approval of the WGII AR5 summary for policymakers (SPM). They involved one or several of the following aspects:

- The definition and interpretation of the term 'detection and attribution';

- The role of observational data, or a lack thereof, representative of both the relevant climate parameter and the impact system for the ability to detect an effect;

- The inference of conclusions from global trends to local effects and vice versa, i.e. the matching of appropriate spatial and temporal scales;

- The differentiation between a sensitivity to weather or manifestations of inter-annual climate variability (e.g. ENSO) and effects of climate change; and

- The relationship between regional climate trends and anthropogenic forcing.

Overall, these misunderstandings can be categorized into those related to the climate, weather and the role of anthropogenic forcing for observed climate manifestations on the one hand, and those concerned with the establishment of causality on the other.

The community working on impacts, adaptation and vulnerability spans a wide range of scientific disciplines; its practitioners and stakeholders do not necessarily possess detailed knowledge about climate variability and change or about how well global and regional climate models perform in 
reproducing and projecting specific climatic traits. Similarly, the approach for detecting changes in climate and attributing them to human influence, which was developed within physical climate science, is not necessarily known beyond their disciplinary borders, nor can these methods be easily transferred into the various settings of different climate change impacts (see Sections 1.2.2 and 1.3.1).

The aggregation of variables into indicators such as 'global mean temperature' helps to identify and communicate the pressing problem of anthropogenic interference with the climate system. However, such highly aggregated indicators are basically meaningless for local processes. Difficulties arise from reversing the construction of 'global climate change' (Hulme 2010), i.a. attributing the effects of changes observed locally to global processes defined by abstract indicators.

Obviously, to ultimately settle the many philosophical considerations and queries attached to the establishment of causality within the scope of this thesis is impossible. But much ground can still be gained for impact attribution by considering two key aspects; one is to simply follow a few, basic steps that consider all the necessary links in the causal chain from observed changes in impact systems to observed changes in the global climate, and possibly the cause of those changes in climate. The other is to clarify what motivates an analysis and whether detection and attribution of observed impacts constitutes an adequate response to that motivation.

This synthesis provides an assessment of the extent to which observed impacts of anthropogenic climate change can presently be detected and attributed worldwide and discusses the implications of its findings for the relevance of detection and attribution research in the context of climate policy and risk, along the five research questions outlined below:

1) What are clear and workable definitions and protocols for detection and attribution in the context of the observed impacts of climate change?

2) Can single extreme weather events and their consequent impacts be attributed to anthropogenic forcing?

3) What is the status of knowledge regarding observed climate change effects worldwide and how has the assessment and evidence base evolved over recent decades?

4) Can impacts of regional and local climate change be attributed to anthropogenic forcing and what are the dominant sources of uncertainty when establishing confidence in such findings?

5) What are the consequences of the challenges and limitations of detection and attribution studies for their use in informing risk assessments and international climate policy? 
First, a clear analytical framework for detection and attribution of climate change impacts is presented, followed by a discussion of extreme event attribution. The contemporary knowledge about observed effects of recent climate change is summarized and linked to anthropogenic forcing of the climate system by means of a multi-step attribution analysis. The uneven global distribution of observed impacts is scrutinized. Touching upon the special requirements of global science-policy assessments, it is debated whether or not impact detection and attribution is a useful indicator of risk. Figure 7.1 provides a graphic overview of the structure of this thesis and how the individual chapters, including this synthesis, relate to the research questions.

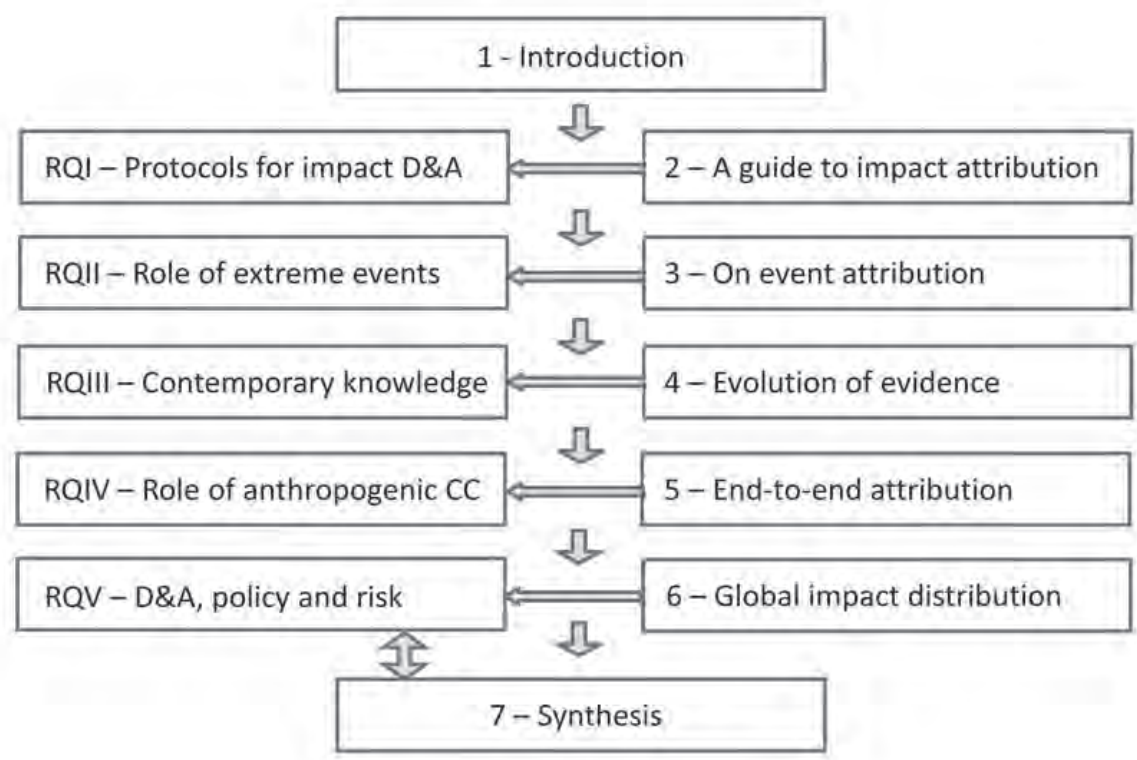

Figure 7.1: Schematic of the structure of this thesis: Each of the Chapters 2-6 (right side, with shorthand titles) addresses one of the five research questions (RQI-V, left side with corresponding shorthand titles) specifically, and Chapter 7 provides a synthesis and additional discussion relevant to RQV. D\&A means detection and attribution, CC stands for climate change.

\subsection{A step-by-step guide to attribution}

This subsection addresses reflects on the establishment of clear and workable definitions and protocols for impact detection and attribution. In the context of climate change research, detection and attribution methodologies have been developed primarily in the field of physical climate sciences. First approaches date back to the 1970s (Chervin et al 1974; Chervin and Schneider 1976; Thompson and Schneider 1982). Today, there is a substantial amount of literature covering a range of similar model-based statistical approaches to the question of how an effect of anthropogenic 
forcing (and, to a lesser extent, its magnitude) can be identified in observational data (Hegerl et al 2007; Hegerl and Zwiers 2011; Bindoff et al 2013).

In contrast, publications that explicitly address methods and approaches for the attribution of observed effects to changes in climate across the wide range of impact systems, are very limited and not comparable. Besides a very small number of studies directly attributing observed effects to anthropogenic forcing, two relevant bodies of literature exist: one is the set of synthesis studies addressing the 'fingerprints' of anthropogenic climate change in large datasets, combining the results of long-term biological and hydrological observational studies (Root et al 2003; Rosenzweig et al 2008; Poloczanska et al 2013), the other comprises a large amount of studies mostly from the fields of glaciology, hydrology, marine and terrestrial ecology, agricultural sciences and public health that address observed changes in climate sensitive systems and examine their respective causes (Cramer et al 2014). While many of these studies effectively perform a detection and attribution analysis, this terminology is not commonly used and the methods applied span a wide range of analytical tools from each study's relevant discipline.

A comprehensive detection and attribution analysis in the context of climate change impacts needs to consider the five steps outlined in Figure 7.2 and described in detail in Chapter 2. In order to detect an effect of observed climate change on a system, its climate must have actually changed and also been observed to have changed for the relevant location and period. This condition distinguishes an impact detection and attribution study from a pure sensitivity analysis that studies the relationship between climate variables and specific parameters of an impact system (e.g. the influence of temperature on crop productivity). An observed local climate trend is not necessarily caused by anthropogenic climate change. Equally, the attribution of an observed (global or regional) trend in climate to human influence does not necessarily signify the existence of a local trend. The difference between the estimation of sensitivity to weather and the observation of an impact of climate change must be made very clear. This applies especially with regard to manifestations of climate variability, such as severe drought or storms.

It is often stated that the detection of a change does not involve consideration of the cause of that change (cf. the detection definition given by Hegerl et al (2010) in Table 1.4). However, this is only true if 'no change beyond natural variability' is implicitly assumed to be the normal state of any system. This assumption may be robust for some systems and situations, such as ecosystems or river flow in undisturbed conditions, but is not applicable in others. 


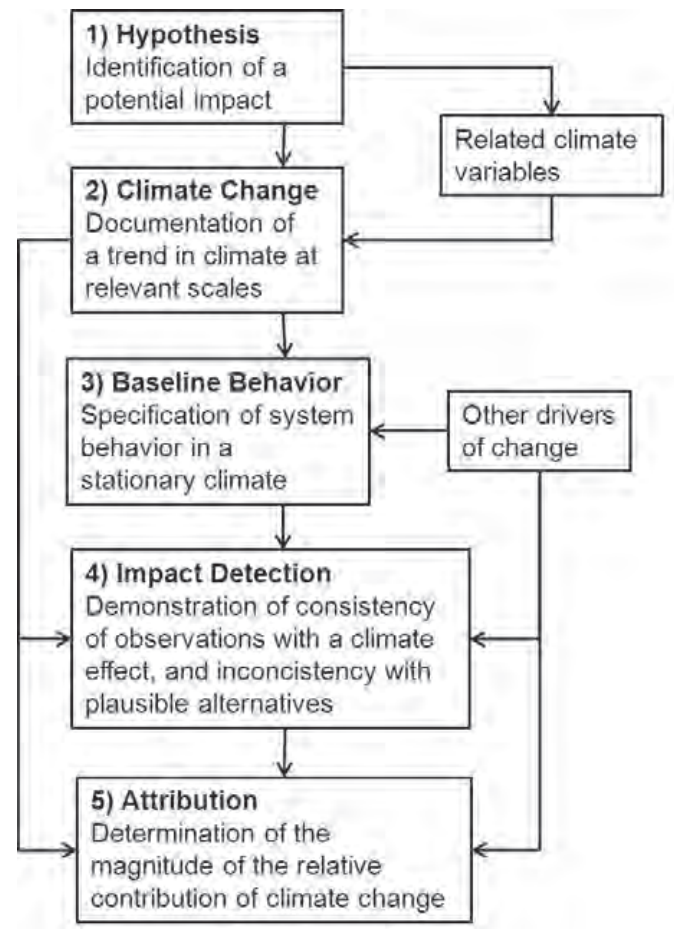

Figure 7.2: The five steps of a comprehensive impact detection and attribution analysis.

Any analysis of cause and effect needs to define the system's boundaries, the drivers considered and potential confounders such as monitoring or modelling errors or unspecified drivers. For the climate system, at least in commonly used global and regional climate models, the system definition seems clear; it involves the separation of external forcing, which can be natural or anthropogenic, and natural variability emerging from internal dynamics of the system. Again, many impact systems involve some ambiguity regarding what is considered external or internal to that system, what would consequently be considered a change and how 'internal variability' would hence be defined. For example, should the current cultural landscapes of central Europe be considered the 'normal system state' or should that rather be the state of some decades or even centuries ago - and what would consequently be the role of human activities other than greenhouse gas emissions? Would habitat fragmentation through infrastructure and urban development, fire suppression and increased nutrient input be considered external forcing, or would their effects constitute part of internal variability? And if the former, does that mean that ecosystems exclude humans?

While the above considerations about internal dynamics apply to most systems, they are more relevant for systems that are actively managed or heavily affected by human activities. In such systems, detection of a 'change' (regardless of its cause) against an assumed baseline of 'no change' 
is futile - coastlines degrade, food production rises, economic output increases, the body size of wild fish decreases and forests degrade - whether the climate changes or not. Also, should the effect of several external drivers cancel each other out, no change will be detected despite the ongoing effect of climate change. Therefore, the explicit consideration of cause and effect is an implicit part of any impact detection analysis.

A mere correlation is not sufficient to detect an impact - it has to be shown that the observed change is consistent with the expected response to the climate trend and less consistent with all plausible responses to non-climate drivers alone. In other words, the addition of a climate driver needs to improve the fit of the model representing the behaviour of the impact system in a statistically significant way. In situations that are not amenable to statistical testing, a thorough evaluation of the role of other drivers and potential confounding factors such as measurement errors or data bias must be provided to support the detection of a climate change impact. A comprehensive qualitative analysis is particularly essential if it is not possible to perform a rigorous quantitative analysis of long-term data.

Impact attribution involves the assessment of the magnitude of the climate change contribution to overall change in relation to the contribution from other drivers. Impact detection implies that climate change has had at least a minor role in the observed outcome. Assessing the magnitude of the contribution of climate change to an impact is a separate step within a detection and attribution exercise. A key challenge for all attribution exercises lies in accounting for the non-additive effects of multiple drivers interacting on several temporal and spatial scales (Parmesan et al 2013; Oliver and Morecroft 2014).

Detection and attribution of climate change impacts provides the most complete and consistent analysis possible of the cause-effect chain, i.e. combining all possible sources of information in a coherent evaluation. Detection and attribution analysis can be a powerful tool in understanding how and why our world is changing; albeit the cost of obtaining the necessary observations and understanding, something that remains poor in many areas, can be prohibitive. Cases exist where data is insufficient to detect an impact, although given climate trends and known sensitivity strongly suggest that climate change will have affected the system. While it is imperative to include evidence from all types of sources and analyses and to apply the full range of methods, both quantitative and qualitative, to establish causality, observational evidence that demonstrates a long-term effect is needed for impact attribution. Or to put it another way - it is impossible to attribute something that has not been detected. 
Within the context of impact attribution, climate change is usually one of many drivers of change and not (yet) necessarily the most prominent one. Consequently, research into past effects will have to focus on the interaction of different drivers of change. By doing so, research dedicated to the detection and attribution of impacts can contribute to increasing resilience in a multi-stressor world and reduce uncertainty about compound impacts.

\subsection{Impact attribution in the context of climate variability and extreme weather events}

This subsection addresses the question of whether single extreme weather events and their consequent impacts can be attributed to anthropogenic forcing. Direct responses to short-term climate variability (e.g. harvest failure due to drought) are often more easily detected than responses to gradual changes in the climate. However, long-term changes in climate variability are much more difficult to detect than changes in climate means; averaging across space and time reduces the 'noise' resulting from internal variability of the system. Consequently, confidence in detection is usually higher for changes in average characteristics of weather and climate, such as the statistical artefact 'global mean temperature'. This, in turn, means that it is not currently possible to directly attribute some of the most pronounced adverse effects relating to the climate (i.e. those caused by extreme weather) to anthropogenic climate change even though these effects might be consistent with what one would expect to see happening under a changing climate. Note that a longterm change in the rate or intensity of extreme weather, i.e. a change in climate variability, constitutes a change in climate, but not necessarily an effect of anthropogenic forcing.

Adverse impacts for both human and natural ecosystems are often driven by extreme weather - the manifestation of climate variability. In conjunction with socioeconomic factors determining vulnerability, (natural) climate variability may lead to climate-related disasters, such as the destruction of physical infrastructure during severe floods, increased mortality and morbidity in the aftermath of a tropical cyclone or extended drought leading to reduced harvest. For many types of extreme weather, understanding of the physical process suggests an increase in intensity or frequency as a result of anthropogenic forcing. Such increases have already been detected in observational datasets for extreme heat and heavy precipitation, while remaining less conclusive for other types of extremes (see also Section 1.4).

Detecting and attributing a change in the frequency of a certain type of event to external forcing of the climate system is generally possible (though often very challenging due to the sparse data), but the same is not necessarily true for individual events (see Chapter 3). Nevertheless, 'event attribution' studies that assess the role of anthropogenic forcing in the occurrence or performance of an individual weather extreme, are being published more and more frequently (Peterson et al 
2013b; Herring et al 2014). Still, attribution information is only available for a small set of events and a bias in case selection has been acknowledged (Hulme 2014). Recognizing the stochastic nature of the problem, scientists have turned to describe the human influence on the manifestation of extreme weather probabilistically. Most studies focus on a variation of a measure called 'fraction attributable risk'; this corresponds to the shift in rates of a certain type of extreme weather occurring under current climate conditions as compared to a hypothetical, stable natural climate.

Chapter 3 outlines a statistical formulation of the attribution of a single event to climate change. This formulation is then used to provide a confidence interval for the attributable risk $p$, i.e. to provide an interval estimate of $p$ that specifies a range within which the true parameter is estimated to lie with a certain level of confidence.

Formulating single-event attribution as outlined above raises two fundamental issues. First, there is an underlying dichotomy to the concept of attributable risk between events that would and would not have occurred in the absence of climate change. This dichotomy makes sense in various contexts, including epidemiology; for example, some cases of lung cancer are caused by smoking while others are not - here, it is natural to ask about the risk of lung cancer attributable to smoking. What is not so clear, however, is to what extent attributable risk makes sense in the context of climate change. While the effects of smoking are confined to the smokers (and perhaps those around them), the effect of climate change is much more pervasive and the notion that, once the climate has changed, some weather events would have occurred exactly as they did in its absence, is untenable (cf. Trenberth 2012). This by no means implies that a change in the rate of events cannot be attributed to anthropogenic forcing, only that the superposition argument on which attributable risk is based is probably not tenable.

Second, even if the notion of attributable risk makes sense in the context of climate change, the quantity $p$ is simply a function of the rates of events before and after climate change and is not particularized to an individual event. In other words, the attributable risk is the same for all postclimate-change events. In this sense, determining $p$ is not really single-event attribution.

Applying the confidence interval, uncertainty regarding attributable risk can clearly remain high unless both the number of observed events and the effects of climate change, i.e. the change in rate, are large. This is true even if the events are unprecedented in the pre-climate change record.

Of course, the statistical challenges involved in confidently detecting trends in very rare events is well known to those working on event attribution - the question, therefore, is rather how to frame robust statements about the role of anthropogenic forcing in the occurrence of a specific event. Unusual weather events are often accompanied by media queries about the role of anthropogenic 
climate change. Some stakeholders declare events such as Super Storm Sandy or Typhoon Haiyan a consequence of global warming in order to engage policymakers and the wider public in the fight against climate change.

From a communication perspective, the attempt to benefit from the public attention in the aftermath of climate-related disasters to raise awareness is very reasonable. The scientific community, however, is met with a serious dilemma: framing the results in a statistically strict and scientifically appropriate way runs the risk of them being inaccessible to the general public. The outcome is probably perceived to be more conservative than appropriate under a risk perspective, given the dire consequences of future climate change, the clear scientific understanding of the mechanisms leading to extreme weather and the urgent need to address the problem.

Whether or not anthropogenic climate change is already causing an increase in the occurrence of extreme weather, is definitely a timely and important issue. However, the risk of disastrous consequences of extreme weather is a function of both the hazard probability (i.e. the hazard risk) and the socioeconomic conditions determining the consequences of such a hazard manifesting (i.e. the system's exposure and vulnerability).

In order to detect a trend in the impacts of a certain type of extreme weather, trends in exposure and vulnerability need to be considered (Huggel et al 2013). However, such a framework would be very challenging to develop and implement. For example, disaster loss statistics account for changes in exposure through normalization (Crompton et al 2010; Bouwer 2011). However, such statistics do not usually incorporate specific circumstances, for example the expected decrease in losses due to improved prevention (Nicholls 2011). This is partly due to the fact that statistical 'black box' models are designed to identify overall trends by eliminating the noise in individual records through aggregation (cf. the global mean temperature records). De-trending disaster loss data based on past adaptation (such as better building codes or enhanced fire-fighting technology) might be feasible for specific datasets. However, in most circumstances developing nationally or regionally appropriate 'adaptation curves' would be difficult. Other salient issues include the difficulty of including nonmonetary losses, accounting for the cumulative effect of several events occurring close in time and the comprehensiveness and accessibility of data (Kousky 2013).

In the case of individual events, the contribution of specific factors to the subsequent loss and damage can be discussed in more detail. For example, the 2003 heat wave in Europe had more severe consequences in France than the heat wave of 2006, even though the latter was worse in terms of meteorological conditions. The major change between the two events was the preparation of the public health services and the learning experience of the population as a whole (Fouillet et al 
2008). The evaluation of individual events can therefore contribute to our understanding of the mechanism of loss and damage incurred by extreme weather and help to identify measures to increase resilience. However, no methods currently exist to translate such conceptual or descriptive knowledge into the attribution of cause and effect. Whether or not attribution would be needed (or useful) to inform policy mechanisms that aim to increase resilience and address loss and damage related to climate change is highly controversial (Hulme et al 2011; Verheyen 2012; James et al 2014).

\subsection{Observed effects of climate change on natural and human systems}

In contrast to the unresolved challenge of attributing the impacts of extreme events, ample and robust evidence exists relating observed effects across a wide range of sectors and systems to recent climate change. The latest IPCC report states that the amount of evidence available, the range of impacts observed and their geographical scope has expanded rapidly (Cramer et al 2014). This subsection summarizes the status of knowledge regarding observed climate change effects worldwide and reflects on the evolution of the evidence base over recent decades.

A highlighted before, in order to document the effects of recent changes in climate, these effects must not only have occurred, but they must also have been observed to have occurred. The increase in observed impacts thus reflects two separate developments: first, that of the actual effects of climate change and second, that of our knowledge about such effects. While it may not be possible to comprehensively assess all global impacts of climate change due to gaps in data and uneven national research capacities and efforts, the current knowledge regarding climate change and its development can well be summarized.

In Chapter 4, a review of impacts documented in the three most recent IPCC assessment reports (TAR, AR4 and AR5) illustrates how evidence documenting observed climate change impacts has substantiated over time. Despite some differences in the approaches taken for impact detection and attribution, the findings of the three assessment reports are broadly consistent and the pattern that emerges from the synopsis of the three reports appears to be robust.

Two decades ago, robust evidence for observed effects of climate change was almost exclusively available for the cryosphere (WGMS 1989; Fitzharris et al 1995) and some terrestrial ecosystems in mid to high northern latitudes or mountain regions (Grabherr et al 1994; Myneni et al 1997). In contrast, the impact of climate change has now been documented in all world regions and oceans, for both natural and human systems. Over the last decade, evidence has particularly increased with regard to impacts on marine ecosystems, food production and wildfire regimes (c.f. Figure 4.1). No recent progress has been found in the documentation of the effects of changing sea levels due to 
climate change, despite the attribution of a global increase in extreme sea levels to global mean sea level rise (Menéndez and Woodworth 2010; Woodworth et al 2011). Although the evidence base has improved substantially for the southern hemisphere and developing nations, the global distribution of observed impacts remains uneven (see Figure 7.3). The Arctic region, for example, is a hotspot for observing the impact of climate change.

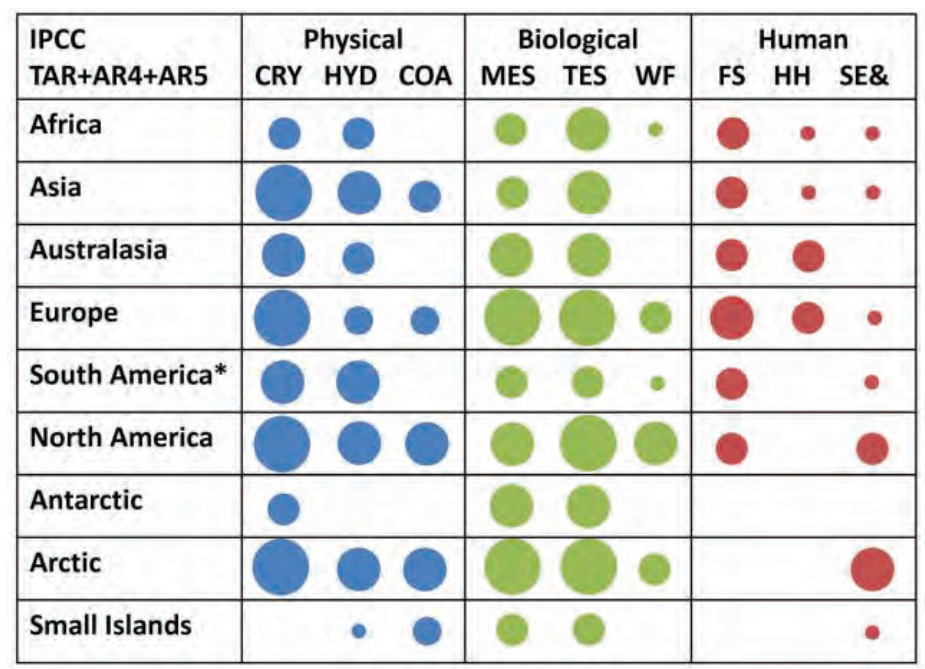

\begin{tabular}{|l|c|}
\hline Legend \\
\hline Zero & \\
\hline Emerging & \\
\hline Moderate & \\
\hline Solid & \\
\hline Extensive & \\
\hline
\end{tabular}

Figure 7.3 Contemporary evidence base representing observed impacts attributed to recent climate change for physical systems [left column: cryosphere (CRY), hydrology (HYD) and coastal processes (COA)], biological systems [centre column: marine (MES) and terrestrial (TES) ecosystems, and impacts of wildfire (WF)], and human and managed systems [right column: food systems (FS), human health (HH), and livelihoods and other socioeconomic variables (SE\&)]. Graphical summary of cumulative evidence from the IPCC's third (TAR), fourth (AR4) and fifth assessment report (AR5) across IPCC regions. Size of circles represents the amount of evidence, from zero to emerging, modest, solid, and extensive. *South America row includes impacts located in Central America.

The vast majority of observed effects are related to temperature change either directly or indirectly, for example mediated through changes in sea ice. Though more and higher quality evidence for impacts related to precipitation is now available, precipitation trends are still less conclusive for many regions than those for temperature. Changes in precipitation are harder to detect due to the low signal-to-noise ratio, in conjunction with high requirements for monitoring density and consequently relatively poor observational records. In addition, impacts of precipitation changes are usually mediated through changes in soil moisture or run-off and both of these variables are not well monitored and subject to change from other factors, such as land use, river management and water withdrawal (Stott et al 2010).

A large fraction of the most robust evidence for the effects of recent climate change is related to the cryosphere or to ecosystems that are highly sensitive to temperature, such as alpine vegetation of 
tropical coral reef systems. While evidence is available across systems and world regions, a substantial part of the observational evidence originates from a specific set of impacts, such as glacier melt or phenology changes in terrestrial ecosystems of temperate and boreal latitudes. Many of these observations served as early indications of climate change and belong to the group of impacts in which climate change is now a dominant driver of pervasive impacts in many regions (see Table 7.1). Such broad impacts are documented for species range shifts and phenology changes in both marine and terrestrial ecosystems, large-scale vegetation changes in the Arctic tundra, increased mass bleaching and mortality in tropical reef-building corals, enhanced glacier melt, mountain and lowland permafrost thaw, changes in patterns of river run-off related to glacier melt, permafrost thaw and changes in seasonal snow storage.

Table 7.1: Extensive impacts of climate change across regions, extracted from Cramer et al (2014). Extensive impacts (dark squares) are defined here as impacts that have been attributed to climate change as a major driver with at least medium confidence at a continental scale, or with high confidence for a subcontinental region of substantial size. Changes in marine ecosystems exclude the effects observed for tropical coral reef systems, as these are singled out as a separate category. South America column includes impacts located in Central America. The global assessment is supplemented with information from the marine, coastal and terrestrial ecosystems chapters of IPCC's WGII AR5 (Pörtner et al 2014; Settele et al 2014; Wong et al 2014).

\begin{tabular}{|c|c|c|c|c|c|c|c|c|c|}
\hline & Africa & Asia & $\begin{array}{c}\text { Austral- } \\
\text { asia }\end{array}$ & Europe & $\begin{array}{l}\text { South } \\
\text { America }\end{array}$ & $\begin{array}{l}\text { North } \\
\text { America }\end{array}$ & Arctic & $\begin{array}{l}\text { Small } \\
\text { Islands }\end{array}$ & Global \\
\hline \multicolumn{10}{|l|}{ Marine ecosystems } \\
\hline Coral bleaching & & & & na & & na & na & & \\
\hline \multicolumn{10}{|c|}{ Terrestrial ecosystems } \\
\hline Glacier melt & & & & & & & & na & \\
\hline Permafrost thaw & na & & na & & na & & & na & \\
\hline River flow change* & na & & na & & & & & na & \\
\hline \multicolumn{10}{|c|}{ *Snow and glacier/permafrost fed rivers } \\
\hline \multicolumn{4}{|c|}{$\begin{array}{l}\text { Extensive impact, i.e. impact documented with at } \\
\text { least medium confidence in a major role of } \\
\text { climate change at continental scale, or high } \\
\text { confidence for a very large sub region }\end{array}$} & \multicolumn{2}{|c|}{$\begin{array}{l}\text { Evidence available, but } \\
\text { below specified } \\
\text { thresholds for } \\
\text { extensive impact }\end{array}$} & & \multicolumn{2}{|c|}{$\begin{array}{l}\text { Not applicable (na) } \\
\text { or no assessment } \\
\text { provided in AR5 }\end{array}$} & \\
\hline
\end{tabular}

Changes in species abundance and distribution consistent with recent climate trends have been shown for many marine species, including fish, invertebrates and phytoplankton in all ocean basins (Poloczanska et al 2013). The improved representation of marine ecosystems in IPCC's AR5 compared to earlier reports is due to a substantial set of additional publications since AR4, but is also the result of a combined effort from the research community to better integrate available knowledge (Richardson et al 2012).

Evidence for impacts on coastal systems remains comparably weak and many of the documented impacts on coastal systems are related to thermokarst and permafrost thaw, as well as changes in sea ice regime in the high northern latitudes, rather than to rising sea levels due to climate change. Global mean sea level is not a reliable or precise indicator of relative sea level change, which is the 
important variable if impacts of sea level rise are to be determined. Relative (or local) sea level changes are subject to a variety of factors, such as natural climate variability influencing currents and wind (e.g. ENSO or the Pacific Decadal Oscillation), vertical land motion due to natural causes (e.g. isostatic uplift) and human activities such as resource extraction (see also WGI AR5 FAQ 13.1 in IPCC 2013b). Alterations of the coastal zone (e.g. mangroves, sediment trapping) influence the impact of higher or extreme sea levels. As sea level is actually an internal factor of the climate system, any change in sea level could be considered climate change. However following the convention applied in Cramer et al (2014), an effect of rising sea levels is considered an impact of climate change if the local rise in sea level that caused the effect is clearly driven by anthropogenic forcing of the climate (not natural or local factors).

The strong representation of effects related to temperature mediated change in snow and ice in the list of observed impacts is one of the reasons for the higher number of observed effects reported from temperate and boreal regions compared to tropical and subtropical regions. Similarly, as changes in spring phenology are amongst the earliest and most widely studied indicators of climate change, the absence of pronounced temperature-related seasons is one of the reasons for the weaker evidence base for terrestrial ecosystems impacts in low latitude regions. In addition, seasonality and ecosystem processes in tropical and subtropical climates are mainly controlled by precipitation, and the detection and attribution of regional precipitation changes remains difficult. Other important reasons for the reduced representation of result from developing countries include a lack of long-term data and extensive monitoring networks, less developed research capacities, issues of data accessibility and the difficulty to assess literature in languages other than English.

In spite of robust evidence in some human sectors and systems, large gaps in our understanding of how important sectors of human societies respond to past climate subsist. Often, information is lacking even in the form of econometric (or other) data on their sensitivity (Deschenes 2013; Houser et al 2014). Another major reason for the weak representation of human systems lies in the fact that humans are remarkably adaptable and often make adjustments in response to risks or impacts. In addition, humans operate in a complex world where many factors change simultaneously. Consequently, for many climate change impacts on human and managed systems detectable today, climate change plays a relatively minor role in comparison with other drivers of change.

Caution must be applied when extrapolating future impacts of (anthropogenic) climate change from these results. Past changes in climate are still comparatively slow and to a large extent within the range experienced by natural climate variability; the effects incurred by recent climate change are therefore a poor predictor for the impacts of future anthropogenic climate change. Accelerating 
rates of climate change and the crossing of important climate thresholds as expected in the future would substantially alter the impact of climate change.

Furthermore, future manifestations of anthropogenic climate change need not be similar to those experienced today. For example, whether a trend towards more frequent very cold weather occurs in parts of North America due to a change in the jet stream and Arctic amplification is still debated (Fischer and Knutti 2014). Such processes could occur as a consequence of anthropogenic forcing and would have very different consequences from those expected to be incurred from gradual winter warming. Another important factor is the prevalence of adaptation measures, in particular for human and managed systems. Contrary to what is to be expected in the future, past responses to climate change may be largely unaffected by planned adaptation to (anthropogenic) climate change, although autonomous adaptation will have taken place in particular in climate sensitive sectors such as agriculture (Thomas et al 2007; Wood et al 2014). Also, social and economic processes unrelated to climate change have improved adaptation to the current climate and climate variability and will likely continue to do so. For example, Barreca et al (2012) report on the remarkable decline in the temperature-mortality relationship in the United States throughout the 20th century, largely due to the adoption of residential air conditioning. However, before turning to the question to what extent the detection and attribution of observed effects of climate change can actually inform about and guide responses to future changes in climate, the next section addresses the relationship between observed impacts of regional climate change and anthropogenic forcing.

\subsection{The relationship between observed impacts and anthropogenic forcing}

This subsection examines whether and to what extent the impacts of regional and local climate change can be attributed to anthropogenic forcing and identifies the dominant sources of uncertainty when establishing confidence in such findings. For large parts of the community studying climate change and its impacts, as well as for many stakeholders, the term 'attribution' is perceived as a synonym for 'attribution to anthropogenic forcing'. As one of the key motivations for detection and attribution research is to provide reliable information for the UNFCCC, this end point has often been considered to be the main goal (Zwiers and Hegerl 2008). The assessment of the relative role of anthropogenic versus natural forcing in observations provides a means to estimate whether recent and current impacts might be expected to persist and to calibrate predictions of future impacts. However, as has been shown in this work, it is often very difficult to detect climate change effects in observed records and to disentangle the impacts of climate change from those of other drivers of change. Clearly, the attribution of observed effects to anthropogenic climate change adds another layer of complexity to an already challenging exercise. 
In Chapter 5, the recent assessment of the observed impacts of regional climate trends provided by the IPCC'S AR5 is extended by adding a climate attribution step that evaluates the role of anthropogenic forcing in the observed climate trend corresponding to each impact. That climate attribution step is centred around the comparison of observed variations in the climate against our expectations of how the climate should have changed. The latter is developed through the understanding of how the climate might respond to external drivers, as expressed in global circulation models (Hegerl et al 2010; Hegerl and Zwiers 2011). In addition to this comparison, the adequacy of the observational and modelled input to the analysis is also considered (Figure 7.4, Annex A).

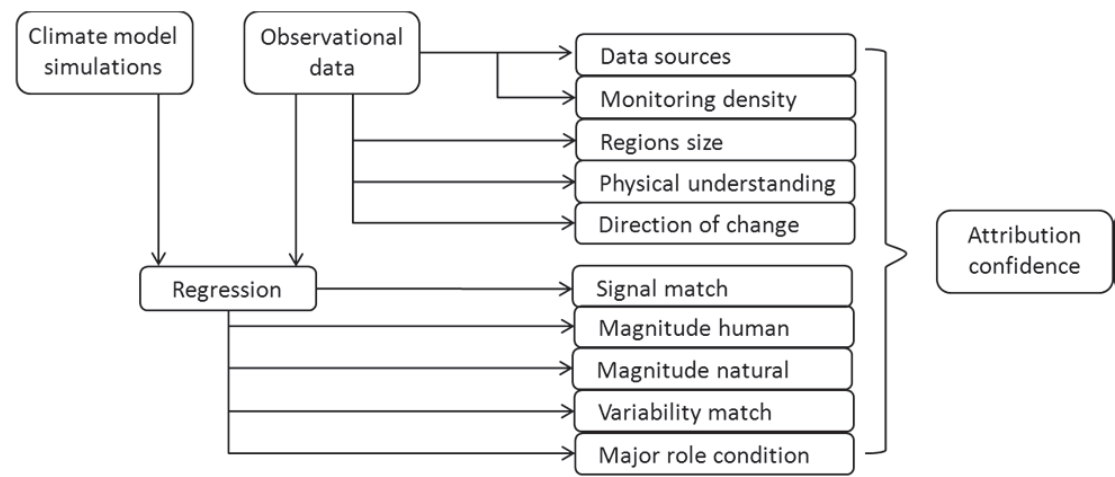

Figure 7.4: Schematic of the algorithm assigning confidence to climate attribution

The algorithm applied for the estimation of confidence in the role of anthropogenic forcing for a given aspect of climate change uses various combinations of the following input:

- Information about the relevant climate variable(s), the direction of the observed change, the relevant season and the spatial extent;

- Multiple gridded observational products;

- Simulations of the climate system from multiple models, driven with all known types of forcing, both anthropogenic and natural, and with natural forcing only; and

- Many years of simulations of dynamical climate models with no variations in external drivers beyond the annual cycle to reproduce internal natural variability.

A metric $c$ is assigned a starting value that depends on the number of data sources available and is then left unchanged or reduced based on a series of tests that consider the adequacy of the input data sources and the agreement between observed changes and our expectations based on processbased modelling, conducted via a linear regression. The metric $c$ is multiplied with a factor $\mu \leq 1$ for each test (see Annex A for a detailed description and numerical representation). The resulting value 
$c_{m}$ is then converted into confidence levels ranging from 'none' to 'very high', and the climate attribution assessment is defined as follows: if the confidence in attribution of a major role is at least medium then that assessment is adopted, otherwise the 'at least a minor role of anthropogenic forcing' (in the following 'minor role' for brevity) assessment is adopted. Based on the outcome of that algorithm, the patterns of attribution confidence for both the impact and the climate aspect are compared, including the analysis of the role of spatial scales and the relevant climate driver. Both assessments are then combined in a tentative multi-step attribution assessment by applying a simple minimum approach.

Anthropogenic forcing was evaluated to have a major role in the observed climate trends in 72 out of 118 cases (i.e. $61 \%$ of total) and a minor role $(25 \%)$ or no role (14\%) in the remaining 46 cases. For about $70 \%$ of the impacts that were examined, anthropogenic forcing has been detected to have at least a minor role in the observed climate trend with medium confidence or higher. If limited to temperature (both ocean and atmospheric) - thereby eliminating many none or very low confidence assessments attached to precipitation (see Section 5.3.2.2) - this value rises to $82 \%$ (84 out of 102), with $71 \%$ representing a major role (72 out of 102$)$.

Climate attribution confidence is higher than impact attribution confidence in particular for effects reported as a consequence of atmospheric temperature change and, to a slightly lesser degree, of ocean temperature change. Thus a conclusion that might be gleaned from this analysis concerning impacts mediated by temperature change is that it is feasible to extend the rigorous impact detection and attribution analysis available for the effects of regional climate trends to the effect of anthropogenic climate change and thus fill the gap noted in the AR5.

In contrast, the confidence in impact attribution is generally higher than the confidence in climate attribution for effects related to precipitation, as the signature of anthropogenic influence remains hardly detectable for changes in precipitation. The majority of assessments related to precipitation fail the first test for consistency between climate trends reported in AR5 and those found in the global observational data products used. This hints at the limited ability of general circulation models (GCMs) to reproduce spatial and temporal precipitation patterns correctly, which is largely due to the fact that precipitation is highly variable and the direct response to anthropogenic forcing relatively weak. However, another cautious interpretation suggests that the sensitivity to short and medium term fluctuations in precipitation is not necessarily a good indicator for exposure to longterm anthropogenic climate change. Ultimately, improved understanding of the role of anthropogenic forcing for impacts of changes in regional climate requires more rigorous analyses of the crucial role of climate compared to other factors in many different local impacts. 
A second central finding, based on the analysis of the combined climate and impact attribution steps for the reported impacts individually, is the fact that no direct relationship can be inferred between confidence in impact attribution and confidence in climate attribution (see Figure 7.5).

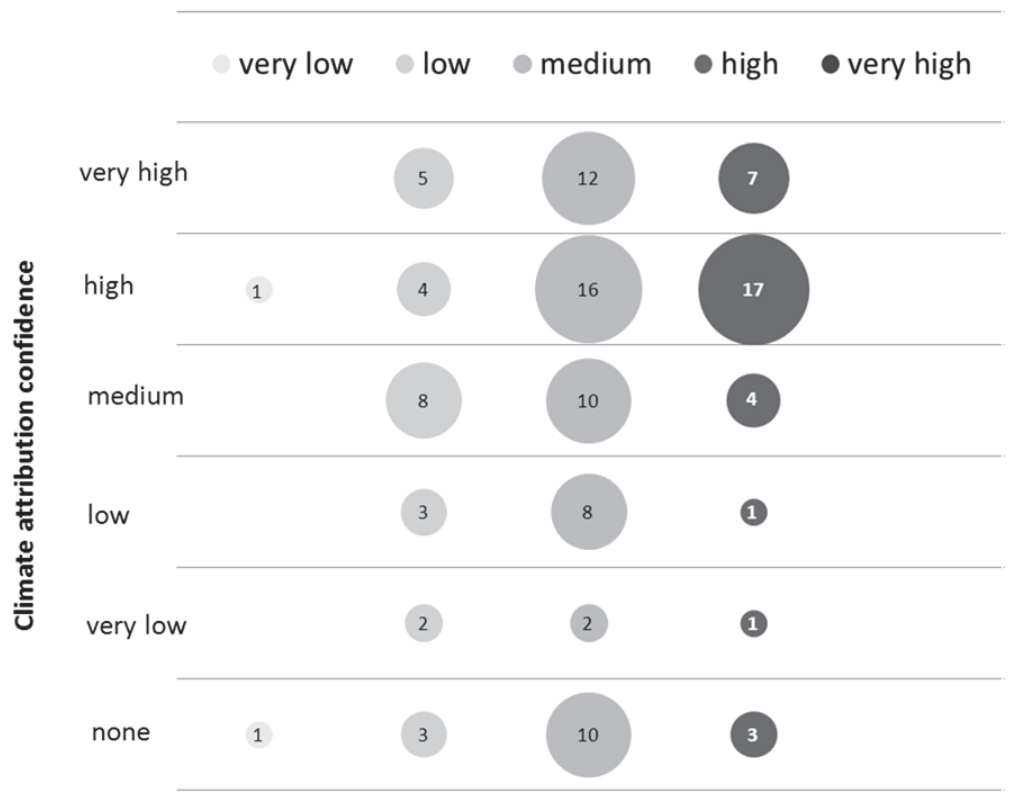

Impact attribution confidence

Figure 7.5: Distribution of confidence levels for impact attribution (horizontal axis) and climate attribution (vertical axis) for the 118 impacts analysed in Chapter 5. Circle area indicates the number of assessments in the respective bin.

The lack of a direct relationship between impact and climate attribution contrasts with the public perception of the list of observed impacts reported by IPCC WGII, which was prominently displayed in a world map showing regional scale and localized impacts (IPCC 2014d, see Figure 6.1). The fact that the icons on that map symbolize observed impacts attributed to recent regional trends in climate, regardless of the role of anthropogenic climate change for those trends, has often been lost or at least under-reported in the media. This led to blind extrapolation from confidence in impact attribution to the role of anthropogenic forcing.

Such extrapolation seems plausible to a certain extent: observed global warming has been attributed to emissions, and that warming is expected to be broadly uniform across the globe. The need for local, seasonal and shorter-period detection and attribution analyses may therefore not be completely evident. Indeed, Christidis et al (2012) estimate distributions of regional annual mean 
temperatures for recent years under actual and hypothetical natural scenarios by using results of analyses conducted at coarser spatial scales to infer the more local information (Christidis et al 2010b). However, the relevant assumptions break down for climate variables with more heterogeneous trends, such as the timing of the rain season onset. Also, the information content of most studies exploring the role of anthropogenic emissions on historical climate change is incomplete, as they fail to explicitly address the relevance of unquantified factors, such as the accuracy of the observational data product (Jones et al 2013). In this respect, it is central to understand that detection and attribution analysis provides information about the state of knowledge constrained by factors such as monitoring records and model performance. Therefore, a failure to detect an effect does not necessarily imply that no such effect exists (see also Chapter 6). To differentiate between absence of evidence and evidence of absence is a central aspect of sciencepolicy assessments that unfortunately tends to get lost in aggregation and communication (see Section 7.5.3).

Still, the analysis in Chapter 5 does confirm an earlier IPCC conclusion that overall, anthropogenic climate change is having a discernible effect on natural systems worldwide; it even extends that conclusion to include human and managed systems. Combining the impact and the climate attribution steps in a multi-step attribution analysis shows that anthropogenic forcing of the climate has a major role in the observed impacts for approximately $42 \%$ of the cases analysed. This number rises to $50 \%$ for a major role of anthropogenic forcing in temperature-related effects and to $75 \%$ for the detection of an anthropogenic effect in temperature-related impacts. These numbers include only combined attribution statements that are made with at least medium confidence. For approximately three-quarters of the impacts specified as extensive, i.e. a major role of climate change for the observed effects has been attributed over a very large area, the climate attribution assessment assigns a major role to anthropogenic forcing with at least medium confidence. For almost $90 \%$ of this group, confidence for climate attribution is high or very high; thus a relationship between impact and climate attribution apparently emerges at higher aggregation levels. However, focussing on effects where anthropogenic climate change is a major driver of change clearly suspends emerging impacts on human and managed systems (Figure 7.6). 


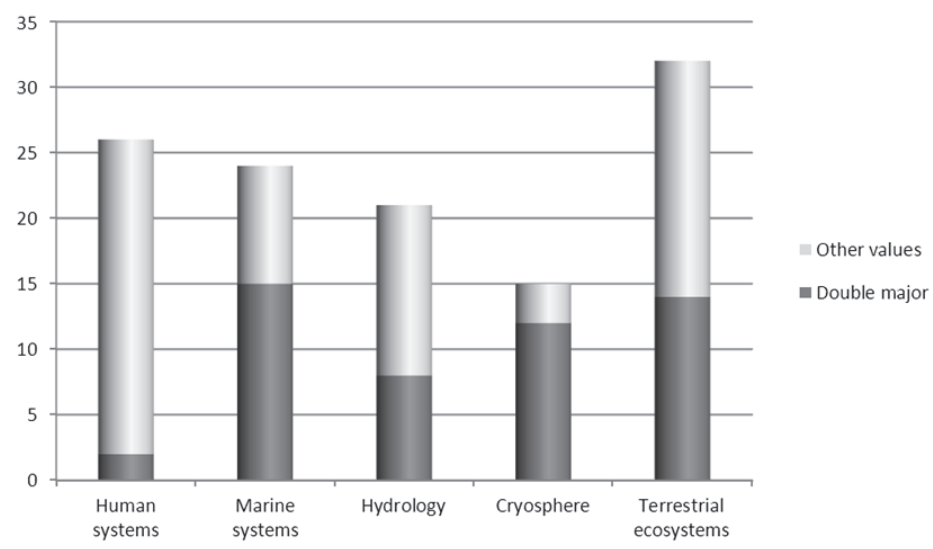

Figure 7.6: Distribution of assessments with both a major role of climate change for the impact observed and a major role of anthropogenic forcing in driving that climate trend (double major), differentiated for main impact systems. Vertical axis indicates the number of assessments. All impacts in the double major group are related to warming of the ocean $(25 \%)$ and the atmosphere $(75 \%)$, none are related to precipitation.

Impacts on the livelihoods of Arctic indigenous peoples are the only incidences where this analysis confirms a major role for anthropogenic climate change for effects observed within human and managed systems. Combined attribution confidence is medium for nine of the twenty-three temperature-related impacts observed in human systems, albeit the role is minor for seven out of those nine cases. The lower confidence in combined attribution for human systems is mainly due to the comparatively minor role of recent climate change in most impacts observed in human and managed systems and the limited confidence in some of those observations.

The climate attribution step is based on the output of (global) GCMs and global gridded sets of observational data. Regional observational products will likely be more accurate (at least for most regions), but the global observational datasets were chosen for consistency. Similarly, the capacity of GCMs to reproduce regional or local climate trends is limited, but downscaled simulation data applicable to attribution analysis is still not available globally. The confidence algorithm recognizes this limitation in several steps, for example, in tuning down confidence for smaller regions.

The confidence algorithm applied to climate attribution is essentially a quantitative approach that is subsequently transferred to a qualitative scale. While it cannot be excluded that a systematic bias has been introduced despite proper calibration, the algorithm and the transfer are still based on the same routine for all assessments and therefore provide a higher degree of standardization compared to the impact side of the assessment. In contrast, the initial assessment by Cramer et al (2014) that served as an input, is a purely qualitative assessment based on expert judgement by scientists with different expertise and backgrounds, and the use of peer review to establish consistency with 
common guidelines between the different assessments. This approach has pioneered outreach and has made it possible to include impact categories that were not formally assessed in earlier reports. Regrettably though, it shares the common weaknesses of expert elicitation, such as a lack of transparency and reproducibility, and a higher degree of subjectivity compared to quantitative assessments. Also, the assessment may not represent the full uncertainty range on the impact side. In the absence of clearly reproducible criteria and hard, quantitative standards, such elicitations tend to avoid extreme values and stay within the 'safe' middle ground. This may explain the even distribution of confidence values on the impact side and the strong representation of 'medium confidence' assessments.

As stated above, the impact attribution assessment focusses on trends in climate - while this approach seems without alternative in the context of a highly-aggregated global assessment, it likely ignores the full range of impacts. For instance, incorporating impacts related to extreme weather within this framework is difficult. Similarly, non-linear responses are hard to capture. However, in the absence of many end-to-end attribution studies examining observed effects across systems and sectors and in a spatially balanced manner, this approach constitutes a plausible systematic method for assessing the influence of anthropogenic forcing on natural and human systems.

\subsection{Detection and attribution in the context of science-policy assessments}

One of the key messages to come out of IPCC's fifth assessment cycle was the fact that the effects of recent climate change are already being observed globally, across sectors and systems, and this was also highlighted in the synthesis report. The results of Chapter 5 provide a link between those observed impacts and anthropogenic climate change, concluding that anthropogenic forcing is implicated in a majority of the observed impacts. However, as discussed in Chapters 2, 4 and 6 of this thesis, important challenges and limitations apply to impact detection and attribution studies. This subsection reflects on the consequences of these findings in the context of global climate change policy. It discusses the relevance of detection and attribution research for adaptation, mitigation and the assessment of risks from climate change. Some general aspects of the art of assessment in the context of global environmental change are summarized and specific challenges for impact detection and attribution are addressed. Finally, an outlook on the future of detection and attribution research in the context of human and managed systems is provided.

\subsubsection{Motivation for detection and attribution research from a climate-policy perspective}

Attributing observed effects to long-term trends in climate helps to identify sensitivities and response patterns and thereby fosters the development of resilient adaptation strategies. 
Adaptation planning may benefit from the in-depth study of cause and effect pertinent to any attribution exercise, in particular when the interaction of various drivers is taken into account; it can also profit from the 'ground testing' that is provided for projections of future impacts made by models or derived from scenario development. Because adaptation is implemented locally, individual studies that examine local circumstances will be more informative than global, aggregated assessments.

The relationship between detection and attribution research and mitigation is more indirect. The main venue will likely be through the use of detection and attribution results as an indicator of risk. The fact that impacts of climate change are already manifesting makes the abstract future risk of climate change more tangible and can raise awareness critical to motivating mitigation actions. In that sense, both local assessments that clearly indicate how climate change affects specific communities, and aggregate global assessments that illustrate the dimension of the risk at a higher level, can be helpful. In addition, the possibility to attribute observed climate-related effects to human influence on the climate may open pathways for litigation or compensation claims related to loss and damage, though considerations related to legal and technical issues currently dominate that debate (Grossman 2003; Verheyen 2012; James et al 2014).

\subsubsection{Detection and attribution of impacts and the assessment of risk}

In recent years, discourse regarding the adverse consequences of future climate change has shifted its focus from the concept of impact to that of risk (Brysse et al 2013; Oppenheimer et al 2014). This shift was facilitated by the close cooperation between the IPCC WGI and WGII communities and the disaster risk community during the preparation of the IPCC special report on managing the risks of extreme events and disasters to advance climate change adaptation (IPCC 2012).

As discussed above, in many human and managed systems the impacts of extreme weather or climate shocks are the rare occasions when a climate-related signal can be detected. However, while the impact of a particular extreme event can be an important indicator of sensitivity to climate, it does not constitute a climate change impact by itself (Allen et al 2007; Stott et al 2013; Hulme 2014).

The scientifically robust attribution of observed impacts to climate change is important for several reasons, including the overall system understanding and the development of resilient strategies for adaptation, as it examines important drivers of change and their interaction. However, as outlined in Chapter 6, a summary of attributed impacts is by no means a complete inventory of the current effects of climate change. Neither does it constitute a suitable standalone indicator of present and future risk of anthropogenic climate change. The manifestation and attribution of a certain climate change effect obviously carries a strong message concerning the risk of that impact occurring. 
However, it does not necessarily provide reliable information about the future risk attached to that effect, given that both future climate change and adaptive responses are uncertain. Also, the fact that an effect has not yet occurred or has not been documented, offers no proof for the absence of such a risk. At the same time, although climate change may act in synergy with other risk factors and will continue to gain importance as the rate and magnitude of climate change increases, the most relevant driver of current risk for human systems from environmental degradation is likely not (global) climate change.

In IPCC'S AR5, the detection and attribution assessment was fed into the aggregated risk assessment represented in the five 'reasons for concern' or 'burning embers' ${ }^{5}$ (Oppenheimer et al 2014). Cramer et al (2014) also used that comprehensive and influential framework (Mahony and Hulme 2012) to synthesize their results for the detection and attribution of observed impacts. However, both failed to address underlying questions of exactly how the observation of an impact of past trends in climate can be related to the additional risk of adverse consequences of anthropogenic climate change now and in the future. Without going into detail here, relevant issues include:

- The (lack of) comprehensiveness of the assessment due to gaps in monitoring records, and consequently the representativeness;

- The role of anthropogenic forcing for the impacts observed was not addressed in the assessment;

- The relationship between past impacts that have manifested (assuming full capacity to observe those impacts) and the risk of future impacts manifesting; and

- The weighing of different impact categories in aggregation, including the balancing of detected and undetected impacts for the risk assessment.

This is not to say that no relationship exists whatsoever. For example, the first of the "burning embers' addresses the risk to 'unique and threatened systems', such as warm water reef-building corals, mountain ecosystems, and the culture and livelihoods of indigenous Arctic peoples and small island communities. Adverse effects have already been observed at the current amount and rate of warming in these systems, which obviously indicates substantial levels of current risk and also has implications for the risk of future consequences. On the other hand, envisaging how observed effects (or a lack thereof) should provide information about the risk of 'large-scale singularities', which are characterized by their non-linear behaviour and sudden onset, remains difficult.

\footnotetext{
${ }^{5}$ The five reasons for concern are: risks to unique and threatened systems, risk of extreme weather events, distribution of impacts, aggregate impacts and risks of large-scale discontinuities (Smith et al 2001; Smith et al 2009). Based on their graphical representation, they are often referred to as the 'burning embers'.
} 


\subsubsection{The art of assessment}

Some of the issues identified above result directly from the requirement to summarize very complex information into general statements or broad indicators, a challenge that is central to science-policy assessments (cf. Hinkel 2011). The scope of this thesis is global and it addresses a complex topic on a highly aggregated level. Also, results of the IPCC's assessments form an integral part of my work. As a consequence, several challenges and limitations pertinent to global science-policy assessments also apply to this thesis. Therefore, important characteristics of such assessments are briefly discussed in this section.

In recent years, global science-policy assessments have been increasingly mandated and carried out in order to provide reliable information to policymakers and the general public. Such assessment processes led to the creation of 'global knowledge' and associated institutions (Hulme 2010) and concurrent implications related to participation, representation and governance (Cash et al 2003; Ford et al 2012; Beck et al 2014; Díaz et al 2015).

Besides the series of IPCC assessments and special reports (www.ipcc.ch/reports), collaboration of international organizations with the research community to produce integrated assessments of key issues in response to complex environmental challenges include the Millennium Ecosystem Assessment (http://www.millenniumassessment.org), the Global Environmental Outlook (http://www.unep.org/geo/) and the International Assessment of Agricultural Knowledge, Science and Technology for Development (McIntyre et al 2009).

While all these assessments differ in scope, procedure and mandate, they all share a few key conceptual aspects. In a wider public policy context, "a scientific assessment applies the judgement of experts to existing knowledge to provide scientifically credible answers to policy-relevant questions" (Leemans 2008). The central requirement of science-policy assessments is to synthesize available knowledge in a form that is accessible and useful for policymakers. Other relevant factors typical for such assessments include a clear mandate by stakeholders (often governments or international bodies representing governments) to evaluate certain questions and to deliver policyrelevant synthesis and integration of the most important results, including an evaluation of options to address the problem. Comprehensiveness of the assessment, based on all relevant literature (and other expertise), including the full range of views on a subject. Explicit statements about uncertainty attached to specific findings, based on the level evidence available, and the agreement. This is achieved by extensive participation of the relevant scientific community, through peer review, and sometimes targeted studies. High transparency of the process and procedures, including selection of experts and the development of assessment criteria and indicators, is key. 
Balance is sought with regard to the representation of scientific disciplines and research areas, and geographic and national origin of the core team of experts and senior coordinators (list adapted from Kowarsch 2014).

Most assessments assemble and synthesize available knowledge and do not perform additional research at a meta-level. As the available knowledge is growing exponentially, a major challenge and opportunity consists in the design and execution of meta-assessments that would facilitate the aggregated assessment of large amounts of literature, including the integration of the growing amount of regional and local studies into a global assessment framework. Possible avenues include the provision of formalized meta-assessments of the literature base, model inter-comparison exercises and broader regional or sectoral assessments using common guidelines.

The production of integrated scenarios, combining assumptions about future socioeconomic conditions and climate policies with climate model projections, is a crucial step in the facilitation of global assessments within the IPCC. Both IPCC WGI and WGIII rely heavily on the outcome of global model inter-comparison projects, such as the coupled inter-comparison project CMIP5 (Taylor et al 2012) or sets of long-term integrated assessment model scenarios (see IAMC AR5 Scenario Database 2014; available at https://secure.iiasa.ac.at/web-apps/ene/AR5DB/). Integrated scenarios combine assumptions about future socioeconomic conditions and climate policies with climate model projections (van Vuuren et al 2011; Kriegler et al 2012; O'Neill et al 2013). Global and regional impact models rely on those scenarios and climate projections as inputs. However, the community working on impacts, adaptation and vulnerability has only recently started to establish common modelling protocols, shared data pools and a consistent framework that could guide an effort similar to those within WGI and WGIII, with the overall goal to produce more comprehensive narratives of global impacts for different levels of warming (Huber et al 2014; Warszawski et al 2014). First results are available mainly in the water (Haddeland et al 2014; Schewe et al 2014) and agriculture sector (Rosenzweig et al 2014). They were also used to assess global hotspots of climate change impacts (Piontek et al 2014).

The advantage of such global modelling exercises lies in their ability to identify robust patterns, systemic feedbacks, trans-regional and trans-sectoral effects and alternative development pathways (Schellnhuber et al 2014). The trade-off consists in a lesser representation of local or regional effects and a failure to deliver 'actionable knowledge', because the coarse resolution does not reliably reproduce local or even regional characteristics. Reanalysis and the comparison of model results with observational databases of relevant impact parameters provide an important instrument for validation and could also contribute to the systematic evaluation of observed effects globally. The creation and maintenance of such databases would also benefit detection and attribution research. 
Notwithstanding the important lessons that can be learned from global model inter-comparisons, one must be careful not to 'put the cart before the horse'. Indeed, some forms of knowledge might become increasingly marginalized due to the fact that they are less accessible for aggregation.

A related challenge consists in the integration of findings of individual (case) studies into broader science-policy assessments. Quantitative meta-analysis derives conclusions based on an array of different studies that report data on the same issue and has been applied as 'quantitative literature review' in global change research (Stanley 2001; Lajeunesse 2010). Issues persist with regard to, for instance, the eligibility criteria, bias correction and the question of whether the goal of the analysis influences the set-up and therefore its outcome. However, the main challenge for science-policy assessments is rather to provide a robust synthesis across a set of studies that report qualitatively on related but ill-defined subjects. Contrary to statistical meta-analysis, there are no standard or agreed methods for conducting syntheses of qualitative research, although a number of approaches do exist (Weed 2005; Campbell et al 2011). To facilitate a systematic approach to all the components of a literature review including the selection process, the assessment of the quality of the research and the clear categorization of findings with a view to aggregation would be decisive to enable future science-policy assessments to draw robust conclusions from the increasingly broader evidence base. Such a systematic approach would probably amplify the workload beyond what is feasible within the current structure of the IPCC. Re-thinking the assessment process in a more fundamental way may be necessary, also in order to enable a more systematic assessment of all scientific and other information sources (Stocker and Plattner 2014; Díaz et al 2015).

\subsubsection{The specific challenges for detection and attribution}

Detection and attribution is, in itself, a fundamentally interdisciplinary exercise. Many difficulties arise, for example, from the adequate use of weather data and climate model output in studies evaluating observed effects of climate change (Auffhammer et al 2013). Other issues concern the requirements regarding observational data and the difficulties to understand the interaction of local and global drivers of change (see Chapter 2). Below, I summarize common challenges that complicate the robust evaluation of the role of (anthropogenic) climate change for observed changes, in particular for human and managed systems.

\subsubsection{Interdisciplinary nature and the integration of social science}

One reason for the weaker documentation of some human systems impacts is the difference in disciplinary approaches for establishing causality between quantitative and qualitative sciences (Stone et al 2013). Detection and attribution standards have been developed by natural scientists 
and usually rely on statistical methods and numerical models (Stone and Allen 2005; Hegerl et al 2010; Hegerl and Zwiers 2011). Some areas of explicit concern in the context of climate change, such as impacts on small-scale farming, informal economies and settlements, livelihoods and poverty, are predominantly qualitatively analysed in scientific literature and do not easily lend themselves to statistical approaches. Indeed, a large part of this literature focusses on current vulnerabilities and future risks in the context of multiple stressors. It does not systematically evaluate observational evidence for effects that have already manifested. The focus of detection and attribution analysis is different from that of vulnerability or impact studies. The latter assess how impacts of future climate change will unfold, based on the sensitivity of a system to climatic factors, expected future climate change and socioeconomic factors delineating vulnerability. In such studies, a system's sensitivity to climate change is often inferred from past responses to climate variability or climatic ranges induced from geographic patterns. However responses to climate variability to not automatically constitute impacts of climate change.

However, a deeper controversy at the heart of the weak representation of qualitative social science also emerges in attribution research and in the IPCC at large. Many social scientists would perceive it as inappropriate to isolate climate change as a single driver from the complex web of factors that determine responses within human societies. Some even accuse such single-driver research of 'neoenvironmental determinism' of human behaviour. A major reason for this criticism is the dominance of Earth systems science in climate change research (Nielsen and Sejersen 2012).

\subsubsection{Multifactorial environments}

To adequately capture the role of climate change in combination with other factors remains a central challenge for impact attribution. Such factors may act as additional stressors, provide resilience or create synergistic effects different from the effect of any individual driver.

In Chapter 2, several examples point to synergistic effects of changes in climate and other drivers, such as the enabling role of the precipitation increases for extension of agricultural activity or the role of warming and weakening winds in triggering the ecosystem shift in Lake Victoria. The contribution from different drivers of change, including adaptation, has also been addressed in the context of extreme impact events (Section 7.2). As highlighted in Chapter 2, the presence of multiple drivers challenge impact attribution compared to climate attribution, especially for systems that are influenced by humans. 


\subsubsection{Observational data gaps and needs}

Scientific attribution of observed impacts to climate change requires time series of observations of sufficient length and quality for the affected system, and for both climatic factors and other important drivers of change, such as land use or economic development. As a result, regions and systems that are well-studied and monitored, exhibit few confounding drivers and a high sensitivity to climate, feature more prominently in the list of attributed impacts (Rosenzweig et al 2007; Rosenzweig and Neofotis 2013; Cramer et al 2014).

Detection and attribution studies are virtually impossible for impacts in some regions due to the absence of such an observational basis. On the climate side, the need for observational data to respond to climate-related concerns has led to the establishment in 1992 of the Global Climate Observing System (GCOS; http://www.wmo.int/pages/prog/gcos/). GCOS has been developed jointly by several UN organisations and the International Council for Science to identify and respond to observational data needs in the context of climate change, including atmospheric, oceanic, hydrological, cryospheric and terrestrial processes. GCOS is based on both in situ measurements and remote sensing; it provides comprehensive information on a range of essential climate variables that can and must be measured to meet the full range of national and international requirements for climate and climate-related observations (Bojinski et al 2014). This endeavour has helped greatly to improve coverage and accessibility of climate data, in particular for the atmosphere and ocean components. However, the human dimensions required for detection and attribution studies are largely absent from the essential climate variables.

The rescue of historic data could contribute to improving the observational basis in understudied areas. Especially since the Satellite age (from 1970 onwards) global coverage of many climate variables has improved considerably, though issues of accessibility and reliability remain. Extending the available time series further into the past and making use of historical data to provide calibration for natural proxies and satellite estimates of surface variables would increase confidence in conclusions regarding local and regional impacts (GCOS 2013). Similarly, analysis of archival footage or historic records of, for example, land use, vegetation composition or coastlines can help to develop robust baselines. To integrate the multiplicity of historical data as represented by the raw observations into processed gridded products and the clear communication of the uncertainties attached to those products constitutes an important research need.

\subsection{Conclusions and outlook}

For both climate and impact research, "understanding observed changes is an essential prerequisite for successful forecasting of future changes" (Stott et al 2010). This thesis augments that 
understanding by clarifying concepts and definitions concerning the attribution of impacts to climate change and extreme weather events, and by providing an analysis of the role of anthropogenic forcing for documented climate change impacts in natural and human systems.

Impacts of recent climate change have been observed across systems and sectors worldwide. The evidence base has improved substantially over the last two decades, but remains geographically unbalanced. The confident detection and attribution of climate change impacts presents a particular challenge in many human and managed systems and in areas with poor monitoring records. Anthropogenic forcing has been shown to play a major role in the majority of observed climate change effects related to temperature, whereas impacts of precipitation changes can generally not be linked to human influence on the climate. Despite the high share of impacts that can be linked to anthropogenic climate change, no direct relationship can be inferred between confidence in impact attribution and confidence in climate attribution. The comparatively minor role of recent climate change in most impacts observed in human and managed systems, and the limited confidence in those observations, currently precludes the attribution of these emerging effects to anthropogenic forcing for the majority of the documented effects.

Impact detection and attribution is fundamentally different from climate attribution. Detection and attribution analysis sets a very high bar in terms of system understanding and data requirements. For many systems that are sensitive to climate historical monitoring is inadequate and will be so for some time, more so in developing countries. However, the uneven distribution of observed impacts across the globe could be improved if the protocols outlined in Chapter 2 were applied in a consistent manner using available data. Also, availability of assessments that attribute impacts to anthropogenic forcing of the climate system could be extended, at least for temperature-driven impacts, applying the method for individual impacted areas used in Chapter 5.

If detection and attribution analysis is to provide a tool for systematic assessment of observed impacts, a framework that accounts for active and planned adaptation needs to be developed and implemented. Similarly, capturing the contribution of anthropogenic forcing to impacts of extreme weather, beyond the probabilistic attribution of hazard risk, is an important research need. Complementary information on current vulnerability and observed climate trends for the respective region would render lists of detected and attributed impacts more accessible in the context of science-policy assessment. Whether or not the observed climate trend is expected to continue or strengthen in the future should be part of such an evaluation in the context of risk.

The scope and nature of available observations strongly influences the possible outcome of any detection and attribution assessment. The more consistent treatment of 'white spots' in our 
knowledge and the communication of the reasons for those spots would help to clarify the relevance of detection and attribution results for policymakers.

The overarching goal of this thesis is to elucidate to what extent the impacts of anthropogenic climate change can be detected and attributed worldwide, in particular within human and managed systems. This is achieved by assessing current impacts of anthropogenic climate change and by addressing the sources of uncertainty, assigning confidence levels and discussing the knowledge differences across a range of relevant climate variables, regions, systems and research disciplines. While the impact of anthropogenic climate change on natural and - to a lesser degree - human systems is confirmed by my analysis, its extent and magnitude cannot be summarized across all climate variables and sectors. 


\section{Bibliography}

ACIA (2005) Arctic Climate Impact Assessment - Scientific Report. Cambridge University Press, Cambridge, UK.

Adelekan IO (2010) Vulnerability of poor urban coastal communities to flooding in Lagos, Nigeria. Environ Urban 22:433-450.

Ahmad Q, Warrick R, Downing TE, et al (2001) Methods and Tools. In: McCarthy JJ, Canziani OF, Leary NA, et al (eds) Climate change 2001: Impacts, Adaptation and Vulnerability. Contribution of Working Group II to the third assessment report of the Intergovernmental Panel on Climate Change. Cambridge University Press, Cambridge, UK, pp 105-134.

Alencar A, Asner GP, Knapp D, Zarin D (2011) Temporal variability of forest fires in eastern Amazonia. Ecol Appl 21:2397-412.

Alexander C, Bynum N, Johnson E, et al (2011) Linking indigenous and scientific knowledge of climate change. Bioscience 61:477-484.

Alexander L V, Arblaster JM (2009) Assessing trends in observed and modelled climate extremes over Australia in relation to future projections. Int J Climatol 29:417-435.

Allen CD, Macalady AK, Chenchouni $\mathrm{H}$, et al (2010) A global overview of drought and heat-induced tree mortality reveals emerging climate change risks for forests. For Ecol Manage 259:660-684.

Allen M (2003) Liability for climate change. Nature 421:891-892.

Allen $M$, Pall $P$, Stone $D$, et al (2007) Scientific challenges in the attribution of harm to human influence on climate. Univ PA Law Rev 155:1353-1400.

Allen MR, Tett SFB (1999) Checking for model consistency in optimal fingerprinting. Clim Dyn 15:419-434.

Arctic Council (2013) Arctic Resilience Interim Report 2013. Arctic Council, Stockholm Environment Institute and Stockholm Resilience Centre, Stockholm, Sweden.

Arnell N, Liu C, Compagnucci R, et al (2001) Hydrology and Water Resources. In: McCarthy JJ, Canziani OF, Leary NA, et al (eds) Climate change 2001: Impacts, Adaptation and Vulnerability. Contribution of Working Group II to the third assessment report of the Intergovernmental Panel on Climate Change. Cambridge University Press, Cambridge, UK, pp 192-233.

Asseng S, Travasso MI, Ludwig F, Magrin GO (2012) Has climate change opened new opportunities for wheat cropping in Argentina? Clim Change 117:181-196.

Auffhammer M, Hsiang SM, Schlenker W, Sobel A (2013) Using Weather Data and Climate Model Output in Economic Analyses of Climate Change. Rev Environ Econ Policy 7:181-198.

Auffhammer M, Ramanathan V, Vincent JR (2006) Integrated model shows that atmospheric brown clouds and greenhouse gases have reduced rice harvests in India. Proc Natl Acad Sci USA 103:19668-19672.

Auffhammer M, Ramanathan V, Vincent JR (2012) Climate change, the monsoon, and rice yield in India. Clim Change 111:411-424.

Auffhammer M, Schlenker W (2014) Empirical Studies on Agricultural Impacts and Adaptation. Energy Econ 46:555-561.

Baldi G, Paruelo JM (2008) Land-Use and Land Cover Dynamics in South American Temperate Grasslands. Ecol Soc 13(2):6.

Barbour AD (1988) Stein's method and Poisson process convergence. J Appl Probab 25:175-184.

Barnett TP, Hasselmann K, Chelliah M, et al (1999) Detection and Attribution of Recent Climate Change : A Status Report. Bull Am Meteorol Soc 80:2631-2659. 
Barnett TP, Pierce DW, Hidalgo HG, et al (2008) Human-induced changes in the hydrology of the western United States. Science 319:1080-1083.

Barreca A, Shapiro JS, Clay K, et al (2012) Adapting to Climate Change: The Remarkable Decline in the U.S. Temperature-Mortality Relationship Over the 20 th Century. MIT Department of Economics Working Paper 12-29. <http://ssrn.com/abstract=2192245>

Barros VR (2010) El cambio climático en Argentina (Chapter 3). Agro y Ambiente. Una agenda compartida para el desarrolo sustentable. Foro de la Cadena Agroindustrial Argentina, Buenos Aires, Argentina.

Battisti A, Stastny M, Netherer S, et al (2005) Expansion of geographic range in the pine processionary moth caused by increased winter temperatures. Ecol Appl 15:2084-2096.

Beaumier M, Ford JD (2010) Food insecurity among Inuit women exacerbated by socio-economic stresses and climate change. Can J Public Heal 101:196-201.

Beck S, Borie M, Chilvers J, Esguerra A (2014) Towards a Reflexive Turn in the Governance of Global Environmental Expertise. GAIA 23(2):80-87.

Bednaršek N, Tarling GA, Bakker DCE, et al (2012) Extensive dissolution of live pteropods in the Southern Ocean. Nat Geosci 5:881-885.

Bennett CM, Dear KGBG, McMichael AJC (2013) Shifts in the seasonal distribution of deaths in Australia, 1968-2007. Int J Biometeorol. 58(5): 835-842.

Berrang-Ford L, Ford JD, Paterson J (2011) Are we adapting to climate change? Glob Environ Chang 21:25-33.

Biasutti M (2013) Forced Sahel rainfall trends in the CMIP5 archive. J Geophys Res 118:1613-1623.

Biasutti M, Giannini A (2006) Robust Sahel drying in response to late 20th century forcings. Geophys Res Lett 33:L11706.

Bindoff NL, Stott PA, AchutaRao KM, et al (2013) Detection and Attribution of Climate Change: from Global to Regional. In: Stocker TF, Qin D, Plattner GK, et al (eds) Climate Change 2013: The Physical Science Basis. Contribution of Working Group I to the Fifth Assessment Report of the Intergovernmental Panel on Climate Change. Cambridge University Press, Cambridge, UK, and New York, USA, pp. 867-952.

Bojinski S, Verstraete M, Peterson TC, et al (2014) The Concept of Essential Climate Variables in Support of Climate Research, Applications, and Policy. Bull Am Meteorol Soc 95:1431-1443.

Bolin B (2008) A history of the science and politics of climate change. The role of the Intergovernmental Panel on Climate Change. Cambridge University Press, Cambridge.

Bouwer LM (2011) Have disaster losses increased due to anthropogenic climate change? Bull Am Meteorol Soc 92:39-46.

Brander K (2010) Impacts of climate change on fisheries. J Mar Syst 79:389-402.

Brander K, Bruno J, Hobday A, Schoeman D (2011) The value of attribution. Nat Clim Chang 1:70-71.

Brink AB, Eva HD (2009) Monitoring 25 years of land cover change dynamics in Africa: a sample based remote sensing approach. Appl Geogr 29:501-512.

Brisson N, Gate P, Gouache D, et al (2010) Why are wheat yields stagnating in Europe? A comprehensive data analysis for France. F Crop Res 119:201-212.

Brown LD, Cai TT, DasGupta A (2001) Interval estimation for a binomial proportion. Stat Sci 16:101117.

Brysse K, Oreskes N, O'Reilly J, Oppenheimer M (2013) Climate change prediction: Erring on the side of least drama? Glob Environ Chang 23:327-337.

Burke M, Hsiang SM, Miguel E (2014) Climate and conflict. NBER Working paper No. 20598. <www.nber.org/papers/w20598> 
Burke MB, Miguel E, Satyanath S, et al (2009) Warming increases the risk of civil war in Africa. Proc Natl Acad Sci USA 106:20670-20674.

Butt A, Buxton M (2009) Peri-urban growth, planning and bushfire in the Melbourne city-region. State of Australian Cities Conference, Perth, WA. Australian Sustainable Cities and Regions Network (ASCRN).

Buxton M, Haynes R, Mercer D, Butt A (2011) Vulnerability to Bushfire Risk at Melbourne's Urban Fringe: The Failure of Regulatory Land Use Planning. Geogr Res 49:1-12.

Campbell R, Pound P, Morgan M, et al (2011) Evaluating meta-ethnography: systematic analysis and synthesis of qualitative research. Health Technol Assess 15:1-164.

Camps JO, Ramos MC (2012) Grape harvest and yield responses to inter-annual changes in temperature and precipitation in an area of north-east Spain with a Mediterranean climate. Int J Biometeorol 56:853-64.

Cardil A, Molina D (2013) Large wildland fires in three diverse regions in Spain from 1978 to 2010. Forest Systems 22:526-534.

Cash DW, Clark WC, Alcock F, et al (2003) Knowledge systems for sustainable development. Proc Natl Acad Sci 100:8086-8091.

Chapman DG (1952) On tests and estimates for the ratio of Poisson means. Ann Inst Stat Math 4:4549.

Chaves LF, Koenraadt CJM (2010) Climate change and highland malaria. Fresh air for a hot debate. Q Rev Biol 85:27-55.

Chen K, McAneney J (2004) Quantifying bushfire penetration into urban areas in Australia. Geophys Res Lett 31:L12212.

Chen M, Xie P, Janowiak JE, Arkin PA (2002) Global Land Precipitation: A 50-yr Monthly Analysis Based on Gauge Observations. J Hydrometeorol 3:249-266.

Chervin RM, Gates WL, Schneider SH (1974) The effect of time averaging on the noise level of climatological statistics generated by atmospheric General Circulation Models. J Atmos Sci 31:2216-2219.

Chervin RM, Schneider SH (1976) On determining the statistical significance of climate experiments with General Circulation Models. J Atmos Sci 33:405-412.

Cheung WWL, Watson R, Pauly D (2013) Signature of ocean warming in global fisheries catch. Nature 497:365-368.

Christidis N, Donaldson GC, Stott PA (2010a) Causes for the recent changes in cold- and heat-related mortality in England and Wales. Clim Change 102:539-553.

Christidis N, Jones GS, Stott $P$ a. (2014) Dramatically increasing chance of extremely hot summers since the 2003 European heatwave. Nat Clim Chang 5:46-50.

Christidis N, Stott PA, Zwiers FW, et al (2012) The contribution of anthropogenic forcings to regional changes in temperature during the last decade. Clim Dyn 39:1259-1274

Christidis N, Stott PA, Zwiers FW, et al (2010b) Probabilistic estimates of recent changes in temperature: a multi-scale attribution analysis. Clim Dyn 34:1139-1156.

Church JA, White NJ (2011) Sea-level rise from the late 19th century to the early 21st century. Surv Geophys 32:585-602.

Clarke H, Lucas C, Smith P (2013) Changes in Australian fire weather between 1973 and 2010. Int J Climatol 33:931-944.

Costa L, Thonicke K, Poulter B, Badeck F-W (2011) Sensitivity of Portuguese forest fires to climatic, human, and landscape variables: subnational differences between fire drivers in extreme fire years and decadal averages. Reg Environ Chang 11:543-551. 
Coumou D, Robinson A, Rahmstorf S (2013) Global increase in record-breaking monthly-mean temperatures. Clim Change 118:771-782.

Cox DR, Isham V (1980) Point Processes. Chapman and Hall, London, UK.

Crain CM, Kroeker K, Halpern BS (2008) Interactive and cumulative effects of multiple human stressors in marine systems. Ecol Lett 11:1304-1315.

Cramer W, Yohe GW, Auffhammer M, et al (2014) Detection and attribution of observed impacts. In: Field CB, Barros VR, Dokken DJ, et al (eds) Climate Change 2014: Impacts, Adaptation and Vulnerability. Part A: Global and Sectoral Aspects. Contribution of Working Group II to the Fifth Assessment Report of the Intergovernmental Panel on Climate Change. Cambridge, UK and New York, NY, USA: Cambridge University Press, Cambridge, UK, and New York, NY, USA, pp. 9791037.

Crate SA (2013) Climate Change and Human Mobility in Indigenous Communities of the Russian North. Project Report. Brookings-LSE Project on Internal Displacement.

Crompton RP, McAneney KJ (2008) Normalised Australian insured losses from meteorological hazards: 1967-2006. Environ Sci Policy 11:371-378.

Crompton RP, McAneney KJ, Chen K, et al (2011) Reply. Weather Clim Soc 3:63-66.

Crompton RRP, McAneney KJ, Chen K, et al (2010) Influence of Location, Population, and Climate on Building Damage and Fatalities due to Australian Bushfire: 1925-2009. Weather Clim Soc 2:300-310.

Darling ES, Cote IM (2008) Quantifying the evidence for ecological synergies. Ecol Lett 11:12781286.

Dash SK, Kjellström T (2011) Workplace heat stress in the context of rising temperature in India. Curr Sci 101:496.

Dell M, Jones BF, Olken BA (2012) Temperature shocks and economic growth: Evidence from the last half century. Am Econ J Macroecon 4:66-95.

Deschenes $O$ (2013) Temperature, human health, and adaptation: A review of the empirical literature. Energy Econ 46:606-619.

Díaz S, Demissew S, Carabias J, et al (2015) The IPBES Conceptual Framework - connecting nature and people. Curr Opin Environ Sustain 14:1-16.

Diemberger H, Hastrup K, Schaffer S, et al (2012) Communicating Climate Knowledge. Curr Anthropol 53:226-244.

Dossou KMR, Glehouenou-Dossou B (2007) The vulnerability to climate change of Cotonou (Benin): the rise in sea level. Environ Urban 19:65-79.

Douglas I, Alam K, Maghenda MA, et al (2008) Unjust waters. Climate change, flooding and the urban poor in Africa. Environ Urban 20:187-205.

Doyle ME, Saurral RI, Barros VR (2011) Trends in the distributions of aggregated monthly precipitation over the La Plata Basin. Int J Climatol 32:2149-2162.

Duarte CM, Agustí S, Wassmann P, et al (2012) Tipping elements in the Arctic marine ecosystem. AMBIO A J Hum Environ 41:44-55.

Dunne JP, Stouffer RJ, John JG (2013) Reductions in labour capacity from heat stress under climate warming. Nat Clim Chang 3:563-566.

Eamer J, Donaldson GM, Gaston a J, et al (2013) Life linked to ice: A guide to sea-ice-associated biodiversity in this time of rapid change. CAFF Assessment Series No. 10. Conservation of Arctic Flora and Fauna, Iceland.

Earnest A, Tan SB, Wilder-Smith A (2012) Meteorological factors and El Niño Southern Oscillation are independently associated with dengue infections. Epidemiol Infect 140:1244-51. 
Ericson J, Vorosmarty C, Dingman S, et al (2006) Effective sea-level rise and deltas: Causes of change and human dimension implications. Glob Planet Change 50:63-82.

Fashae OA, Onafeso OD (2011) Impact of climate change on sea level rise in Lagos, Nigeria. Int J Remote Sens 32:9811-9819.

Fischer EM, Knutti R (2014) Impacts: Heated debate on cold weather. Nat Clim Chang 4:537-538.

Fitzharris BB, Allison I, Braithwaite RJ, et al (1995) The Cryosphere: Changes and Their Impacts. In: Watson RT, Zinyovera M, Moss R, Dokken D (eds) Climate Change 1995: Impacts, Adaptations and Mitigation of Climate Change: Scientific-Technical Analyses. Cambridge University Press, pp. 242-265.

Folland CV, Karl TR, Christy JR, et al (2001) Observed Climate Variability and Change. In: Houghton JT, Ding Y, Griggs DJ, et al (eds) Climate Change 2001: Physical Science Basis. Cambridge University Press, Cambridge, UK, pp. 99-181.

Ford JD, Berrang-Ford L, Paterson J (2011) A systematic review of observed climate change adaptation in developed nations. Clim Change 106:327-336.

Ford JD, Pearce T (2010) What we know, do not know, and need to know about climate change vulnerability in the western Canadian Arctic: a systematic literature review. Environ Res Lett 5:014008.

Ford JD, Vanderbilt W, Berrang-Ford L (2012) Authorship in IPCC AR5 and its implications for content: Climate change and Indigenous populations in WGII. Clim Change 113:201-213.

Fouillet A, Rey G, Wagner V, et al (2008) Has the impact of heat waves on mortality changed in France since the European heat wave of summer 2003? A study of the 2006 heat wave. Int J Epidemiol 37:309-317.

Furrer EM, Katz RW, Walter MD, Furrer R (2010) Statistical modeling of hot spells and heat waves. Clim Res 43:191-205.

GCOS (2013) Workshop on Observations for Adaptation to Climate Variability and Change - GCOS166. World Meteorological Organization.

<http://www.wmo.int/pages/prog/gcos/Publications/gcos-166.pdf>

Gillett NP (2004) Detecting the effect of climate change on Canadian forest fires. Geophys Res Lett 31:L18211.

Giorgi $F(2002)$ Variability and trends of sub-continental scale surface climate in the twentieth century. Part I: observations. Clim Dyn 18:675-691.

Girardin MP, Ali A a., Carcaillet C, et al (2013) Fire in managed forests of eastern Canada: Risks and options. For Ecol Manag Spec Issues Mega Fires 294:238-249.

Gitay H, BrownS, Easterling W, et al (2001) Ecosystems and Their Goods and Services. In: McCarthy JJ, Canziani OF, Leary NA, et al (eds) Climate change 2001: Impacts, Adaptation and Vulnerability. Contribution of Working Group II to the third assessment report of the Intergovernmental Panel on Climate Change. Cambridge University Press, New York, pp. 235342.

Gonzalez P, Tucker CJ, Sy H (2012) Tree density and species decline in the African Sahel attributable to climate. J Arid Environ 78:55-64.

Grabherr G, Gottfried M, Pauli H (1994) Climate effects on mountain plants. Nature 369:448.

Greene AM, Giannini A, Zebiak SE (2009) Drought return times in the Sahel: a question of attribution. Geophys Res Lett 36:L12701.

Gregory PJ, Marshall B (2012) Attribution of climate change: a methodology to estimate the potential contribution to increases in potato yield in Scotland since 1960. Glob Chang Biol 18:1372-1388. 
Grossman DA (2003) Warming up to a not-so-radical idea: tort-based climate change litigation. J Environ Law 28:1-62.

Haddeland I, Heinke J, Biemans H, et al (2014) Global water resources affected by human interventions and climate change. Proc Natl Acad Sci USA 111:3251-6.

Handmer J, Honda Y, Kundzewicz ZW, et al (2012) Changes in impacts of climate extremes. Human systems and ecosystems. In: Field CB, Barros V, Stocker TF, et al (eds) Managing the Risks of Extreme Events and Disasters to Advance Climate Change Adaptation. A special report of Working Groups I and II of the Intergovernmental Panel on Climate Change. Cambridge University Press, Cambridge, UK and New York, NY, USA, pp. 231-290.

Hansen G, Stone D, Auffhammer M (2013) Detection and attribution of climate change impacts - is a universal discipline possible? In Impacts World 2013 Conference Proceedings, pp 438-445.

Hansen J, Ruedy R, Sato M, Lo K (2010) Global surface temperature change. Rev Geophys 48:2010RG000345.

Harris I, Jones PD, Osborn TJ, Lister DH (2014) Updated high-resolution grids of monthly climatic observations - the CRU TS3.10 Dataset. Int J Climatol 34:623-642.

Hartmann DL, Klein AMG, Tank M, et al (2013) Observations: Atmosphere and Surface. In Stocker TF, Qin D, Plattner GK, et al (eds) Climate Change 2013: The Physical Science Basis. Contribution of Working Group I to the Fifth Assessment Report of the Intergovernmental Panel on Climate Change. Cambridge University Press, Cambridge, UK and New York, NY, USA, pp 159-254.

Haynes K, Handmer J, McAneney J, et al (2010) Australian bushfire fatalities 1900-2008: exploring trends in relation to the "Prepare, stay and defend or leave early" policy. Environ Sci Policy 13:185-194.

Hecky RE, Mugidde R, Ramlal PS, et al (2010) Multiple stressors cause rapid ecosystem change in Lake Victoria. Freshw Rev 55:19-42.

Hegerl G, Zwiers F (2011) Use of models in detection and attribution of climate change. Wiley Interdiscip Rev Clim Chang 2:570-591.

Hegerl G, Zwiers F, Tebaldi C (2011) Patterns of change: whose fingerprint is seen in global warming? Environ Res Lett 6:044025.

Hegerl GC, Hoegh-Guldberg O, Casassa G, et al (2010) Good practice guidance paper on detection and attribution related to anthropogenic climate change. In: Stocker TF, Field CB, Qin D, et al (eds) Meeting Report of the Intergovernmental Panel on Climate Change Expert Meeting on Detection and Attribution of Anthropogenic Climate Change. IPCC Working Group I Technical Support Unit, University of Bern, Bern, Switzerland.

Hegerl GC, Zwiers FW, Braconnot P, et al (2007) Understanding and attributing climate change. In Solomon S, Qin D, Manning M, et al (eds) Climate Change 2007: The Physical Science Basis. Contribution of Working Group I to the Fourth Assessment Report of the Intergovernmental Panel on Climate Change. Cambridge University Press, Cambridge, UK and New York, NY, USA, pp 663-745.

Hemp A (2009) Climate change and its impact on the forests of Kilimanjaro. AfrJEcol 47:3-10.

Herring SC, Hoerling MP, Peterson TC, Stott PA (Eds) (2014) Explaining Extreme Events of 2013 from a Climate Perspective. Bull Am Meteorol Soc Vol 95:S1-S96.

Hidalgo HG, Das T, Dettinger MD, et al (2009) Detection and Attribution of Streamflow Timing Changes to Climate Change in the Western United States. J Clim 22:3838-3855.

Hinkel J (2011) Indicators of vulnerability and adaptive capacity: Towards a clarification of the science-policy interface. Glob Environ Chang 21:198-208.

Hockey PAR, Sirami C, Ridley AR, et al (2011) Interrogating recent range changes in South African birds: confounding signals from land use and climate change present a challenge for attribution. Divers Distrib 17:254-261. 
Hoegh-Guldberg O, Hegerl G, Root T, et al (2011) Difficult but not impossible. Nat Clim Chang 1:72.

Houser T, Kopp R, Hsiang S, et al (2014) American Climate Prospectus Economic - Risks in the United States. Rhodium Group, LLC, New York, USA.

Hoyos LE, Cingolani AM, Zak MR, et al (2013) Deforestation and precipitation patterns in the arid Chaco forests of central Argentina. Appl Veg Sci 16:260-271.

Hsiang SM (2010) Temperatures and cyclones strongly associated with economic production in the Caribbean and Central America. Proc Natl Acad Sci USA 107:15367-72.

Hsiang SM, Burke M, Miguel E (2013) Quantifying the influence of climate on human conflict. Science 341:1235367.

Huber V, Schellnhuber HJ, Arnell NW, et al (2014) Climate impact research : beyond patchwork. Earth Syst Dyn 399-408.

Huggel C, Stone D, Auffhammer M, Hansen G (2013) Loss and damage attribution. Nat Clim Chang 3:694-696.

Hulme M (2014) Attributing weather extremes to "climate change": A review. Prog Phys Geogr 38:499-511.

Hulme M (2010) Problems with making and governing global kinds of knowledge. Glob Environ Chang 20:558-564.

Hulme M, O'Neill SJ, Dessai S (2011) Is weather event attribution necessary for adaptation funding? Science 334:764-765.

Hurrell JW, Hack JJ, Shea D, et al (2008) A new sea surface temperature and sea ice boundary dataset for the Community Atmosphere Model. J Clim 21:5145-5153.

Hyatt OM, Lemke B, Kjellstrom T (2010) Regional maps of occupational heat exposure: past, present, and potential future. Glob Health Action 3:1-10.

IPCC (2013a) Summary for Policymakers. In: Stocker TF, Qin D, Plattner GK, et al (eds) Climate Change 2013: The Physical Science Basis. Contribution of Working Group I to the Fifth Assessment Report of the Intergovernmental Panel on Climate Change Cambridge University Press, Cambridge, UK and New York, USA, pp 3-29.

IPCC (2014a) Climate Change 2014: Impacts, Adaptation, and Vulnerability. Part A: Global and Sectoral Aspects. Contribution of Working Group II to the Fifth Assessment Report of the Intergovernmental Panel on Climate Change. Field, CB, Barros VR, Dokken DJ, et al (eds) Cambridge University Press, Cambridge, UK and New York, NY, USA.

IPCC (2014b) Annex XX: Glossary [Agard, J., E. Schipper, J. Birkmann, M. Campos, C. Dubeux, Y. Nojiri, L. Olsson, B. Osman-Elasha, M. Pelling, M. Prather, M. Rivera-Ferre, O. Ruppel, A. Sallenger, K. Smith, A. St Clair, K. Mach, M. Mastrandrea, T. Bilir (eds)]. In: Barros VR, Field CB, Dokken DJ, et al (eds) Climate Change 2014: Impacts, Adaptation, and Vulnerability. Part B: Regional Aspects. Contribution of Working Group II to the Fifth Assessment Report of the Intergovernmental Panel on Climate Change Cambridge University Press, Cambridge, UK and New York, NY, USA, pp 1757-1776.

IPCC (1996) Climate Change 1995. Impacts, Adaptations and Mitigation of Climate Change: ScientificTechnical Analyses. Contribution of Working Group II to the Second Assessment Report of the Intergovernmental Panel on Climate Change. Watson RT, Zinyowera MC, Moss RH, et al (eds), Cambridge University Press, Cambridge, UK.

IPCC (2010) Meeting Report of the Intergovernmental Panel on Climate Change Expert Meeting on Detection and Attribution Related to Anthropogenic Climate Change. Stocker TF, Field CB, Qin D, et al (eds), IPCC Working Group I Technical Support Unit, University of Bern, Bern, Switzerland.

IPCC (2001) Climate Change 2001. Impacts, Adaptation, and Vulnerability. McCarthy JJ, Canziani O, Leary N, et al (eds) Cambridge University Press, Cambridge, UK. 
IPCC (2007a) Climate Change 2007: Impacts, Adaptation and Vulnerability. Contribution of Working Group II to the Fourth Assessment Report of the Intergovernmental Panel on Climate Change. Parry ML, Canziani OF, Palutikof JP, et al (eds) Cambridge University Press, Cambridge, UK.

IPCC (2007b) Summary for Policymakers. In: Parry ML, Canziani OF, Palutikof JP, et al (eds) Climate Change 2007: Impacts, Adaptation and Vulnerability. Contribution of Working Group II to the Fourth Assessment Report of the Intergovernmental Panel on Climate Change. Cambridge University Press, Cambridge, UK, pp 7-22.

IPCC (2012) Managing the risk of extreme events and disasters to advance climate change adaptation. A special report of Working Groups I and II of the Intergovernmental Panel on Climate Change. Field CB, Barros V, Stocker TF, et al (eds) Cambridge University Press, Cambridge, UK.

IPCC (2013b) Climate Change 2013: The Physical Science Basis. Contribution of Working Group I to the Fifth Assessment Report of the Intergovernmental Panel on Climate Change. Stocker TF, Qin D, Plattner GK, et al (eds) Cambridge University Press, Cambridge, UK and New York, NY, USA.

IPCC (2007c) Climate Change 2007: The Physical Science Basis. Contribution of Working Group I to the Fourth Assessment Report of the Intergovernmental Panel on Climate Change. Solomon S, Qin D, Manning M, et al (eds) Cambridge University Press, Cambridge, UK and New York, NY, USA.

IPCC (2014c) Climate Change 2014: Impacts, Adaptation, and Vulnerability. Part B: Regional Aspects. Contribution of Working Group II to the Fifth Assessment Report of the Intergovernmental Panel on Climate Change Barros VR, Field CB, Dokken DJ, et al (eds) Cambridge University Press, Cambridge, UK and New York, NY, USA.

IPCC (2014d) Summary for Policymakers. In: Field CB, Barros VR, Dokken DJ, et al (eds) Climate Change 2014: Impacts, Adaptation, and Vulnerability. Part B: Regional Aspects. Contribution of Working Group II to the Fifth Assessment Report of the Intergovernmental Panel on Climate Change. Cambridge University Press, Cambridge, UK and New York, NY, USA, pp 1-32.

IPCC (2014e) Summary for Policymakers. In: Core Writing Team, Pachauri R, Meyer L (eds) Climate Change 2014: Synthesis Report. Contributions of Working Groups I, II and III to the Fifth Assessment Report of the Intergovernmental Panel on Climate Change. IPCC, Geneva, Switzerland, pp 1-31.

Jagger TH, Elsner JB (2006) Climatology models for extreme hurricane winds near the United States. J Clim 19:3220-3236.

James R, Otto F, Parker H, et al (2014) Characterizing loss and damage from climate change. Nat Clim Chang 4:938-939.

Jaramillo J, Setamou M, Muchugu E, et al (2013) Climate change or urbanization? Impacts on a traditional coffee production system in East Africa over the last 80 years. PLoS One 8:e51815.

Jeffries MO, Overland JE, Perovich DK (2013) The Arctic shifts to a new normal. Phys Today 66:35-40.

Jones GS, Stott PA, Christidis N (2013) Attribution of observed historical near-surface temperature variations to anthropogenic and natural causes using CMIP5 simulations. J Geophys Res 118:4001-4024.

Jovanovic BD, Levy PS (1997) A look at the Rule of Three. Am Stat 51:137-139.

Karoly DJ, Wu Q (2005) Detection of regional surface temperature trends. J Clim 18:4337-4343.

Kennedy JJ, Rayner NA, Smith RO, et al (2011a) Reassessing biases and other uncertainties in seasurface temperature observations since 1850 part 2: biases and homogenisation. J Geophys Res 116(14):D14104.

Kennedy JJ, Rayner NA, Smith RO, et al (2011b) Reassessing biases and other uncertainties in seasurface temperature observations since 1850 part 1: measurement and sampling errors. J Geophys Res 116(14):D14103 
Kjellstrom T, Holmer I, Lemke B (2009) Workplace heat stress, health and productivity - an increasing challenge for low and middle-income countries during climate change. Glob Health Action 2:46-51.

Knutson TR, McBride JL, Chan J, et al (2010) Tropical cyclones and climate change. Nat Geosci 3:157163.

Kolding J, Van Zwieten P, Mkumbo OC, et al (2008) Are the Lake Victoria fisheries threatened by exploitation or eutrophication? Towards an ecosystem based approach to management. In: Bianchi G, Skjodal HR (eds) The Ecosystem Approach to Fisheries. CAB International, Rome, pp 309-354.

Kousky C (2013) Informing climate adaptation: A review of the economic costs of natural disasters. Energy Econ 46:576-592.

Koutsias N, Arianoutsou M, Kallimanis AS, et al (2012) Where did the fires burn in Peloponnisos, Greece the summer of 2007? Evidence for a synergy of fuel and weather. Agric For Meteorol 156:41-53.

Kowarsch M (2014) What are scientific assessments? MCC working paper 4/2014. Mercator Research Institute for Global Commons and Climate Change, Berlin.

Kriegler E, O'Neill BC, Hallegatte S, et al (2012) The need for and use of socio-economic scenarios for climate change analysis: a new approach based on shared socio-economic pathways. Glob Environ Chang 22(4):807-822.

Kroeker KJ, Kordas RL, Crim R, et al (2013) Impacts of ocean acidification on marine organisms: quantifying sensitivities and interaction with warming. Glob Chang Biol 19:1884-96.

Krupnik I, Ray GC (2007) Pacific walruses, indigenous hunters, and climate change: Bridging scientific and indigenous knowledge. Deep Res Part II-Topical Stud Oceanogr 54:2946-2957.

Lajeunesse MJ (2010) Achieving synthesis with meta-analysis by combining and comparing all available studies. Ecology 91:2561-4.

Lebel T, Ali A (2009) Recent trends in the Central and Western Sahel rainfall regime (1990-2007). J Hydrol 375:52-64.

Leemans R (2008) Personal experiences with the governance of the policy-relevant IPCC and Millennium Ecosystem Assessments. Glob Environ Chang 18:12-17.

Lehman J, Mugidde R, Lehman D (1998) Lake Victoria plankton ecology: Mixing depth and climatedriven control of lake condition. In: Lehman JT (ed) Environmental Change and Response in East African Lakes. Monographiae Biologicae Volume 79. Springer, Netherlands, pp 99-116

Lewis SC, Karoly DJ (2013) Anthropogenic contributions to Australia's record summer temperatures of 2013. Geophys Res Lett 40:3705-3709.

Li S, Tao H, Xu Y (2013) Abiotic determinants to the spatial dynamics of dengue fever in Guangzhou. Asia Pac J Public Health 25:239-47.

Lobell DB, Bänziger M, Magorokosho C, Vivek B (2011a) Nonlinear heat effects on African maize as evidenced by historical yield trials. Nat Clim Chang 1:42-45.

Lobell DB, Field CB (2007) Global scale climate - crop yield relationships and the impacts of recent warming. Environ Res Lett 2:14002.

Lobell DB, Hammer GL, McLean G, et al (2013) The critical role of extreme heat for maize production in the United States. Nat Clim Chang 3:497-501.

Lobell DB, Schlenker W, Costa-Roberts J (2011b) Climate trends and global crop production since 1980. Science 333:616.

Loiselle S, Cózar A, Adgo E, et al (2014) Decadal trends and common dynamics of the bio-optical and thermal characteristics of the African Great Lakes. PLoS One 9:e93656. 
Lott FC, Christidis N, Stott P a. (2013) Can the 2011 East African drought be attributed to humaninduced climate change? Geophys Res Lett 40:1177-1181.

Mack MC, Bret-Harte MS, Hollingsworth TN, et al (2011) Carbon loss from an unprecedented Arctic tundra wildfire. Nature 475:489-492.

Magrin GO, Marengo JA, Boulanger J-P, et al (2014) Central and South America. In: Barros VR, Field CB, Dokken DJ, et al (eds) Climate Change 2014: Impacts, Adaptation, and Vulnerability. Part B: Regional Aspects. Contribution of Working Group II to the Fifth Assessment Report of the Intergovernmental Panel on Climate Change. Cambridge University Press, Cambridge, UK and New York, NY, USA, pp 1499-1566.

Magrin GO, Travasso MI, Baethgen WE, et al (2007) Past and Future Changes in Climate and their Impacts on Annual Crops Yield in South East South America. IPCC TGICA Expert Meeting Integrating Analysis of Regional Climate Change and Response Options, Nadi,Fiji. Meeting Report. Intergovernmental Panel on Climate Change (IPCC), Geneva, Switzerland, pp 121-124.

Magrin GO, Travasso MI, Rodríguez GR, et al (2009) Climate change and wheat production in Argentina. Int J Glob Warm 1:214-226.

Magrin GO, Travasso MI, Rodríguez GR (2005) Changes in Climate and Crop Production During the 20th Century in Argentina. Clim Change 72:229-249.

Mahony M, Hulme M (2012) An Exploration of the IPCC's "Burning Embers" Diagram. Spontaneous Gener A J Hist Philos Sci 61:75-89.

Maranz S (2009) Tree mortality in the African Sahel indicates an anthropogenic ecosystem displaced by climate change. J Biogeogr 36:1181-1193.

Marin A (2010) Riders under storms: Contributions of nomadic herders' observations to analysing climate change in Mongolia. Glob Environ Chang 20:162-176.

Marzeion B, Cogley JG, Richter K, Parkes D (2014) Attribution of global glacier mass loss to anthropogenic and natural causes. Science 345:919-921.

Mastrandrea MD, Field CB, Stocker TF, et al (2010) Guidance notes for lead authors of the IPCC Fifth Assessment Report on consistent treatment of uncertainties. Intergovernmental Panel on Climate Change (IPCC), Geneva, Switzerland.

Matsuura K, Willmott CJ (2012) Terrestrial Air Temperature and Precipitation: 1900-2010 Gridded Monthly Time Series (v.3.01). University of Delaware. <http://climate.geog.udel.edu>

McAneney J, Chen K, Pitman A (2009) 100-years of Australian bushfire property losses: is the risk significant and is it increasing? J Environ Manage 90:2819-22.

McDowell JZ, Hess JJ (2012) Accessing adaptation: Multiple stressors on livelihoods in the Bolivian highlands under a changing climate. Glob Environ Chang 22:342-352.

McIntyre BD, Herren R, Wakhungu J, Watson RT (Eds) (2009) International assessment of agricultural knowledge, science and technology for development (IAASTD): global report. Island Press, Washington DC, USA.

Meehl GA, Tebaldi C (2004) More intense, more frequent, and longer lasting heat waves in the 21st century. Science 305:994-997.

Menéndez M, Woodworth PL (2010) Changes in extreme high water levels based on a quasi-global tide-gauge data set. J Geophys Res Ocean 115:C10011.

Meng Q, Hou P, Lobell DB, et al (2013) The benefits of recent warming for maize production in high latitude China. Clim Change 122:341-349.

Menzel A, Sparks TH, Estrella N, et al (2006) European phenological response to climate change matches the warming pattern. Glob Chang Biol 12:1969-1976. 
Mills GA, Timbal B, Walsh K (2008) Assessing the impact of climate change on extreme fire weather in southeast Australia. Centre for Australian Weather and Climate Research. Technical Report No7. CSIRO and the Australian Bureau of Meterology, Melbourne, Australia.

Mitchell J, Karoly D, Hegerl G, et al (2001) Detection of climate change and attribution of causes. In Houghton J, Ding Y, Griggs DJ, et al (eds) Climate Change 2001: The Scientific Basis. Contribution of Working Group I to the Third Assessment Report of the Intergovernmental Panel on Climate Change. Cambridge University Press, Cambridge, UK, pp 696-738.

Mouhamed L, Traore SB, Alhassane A, Sarr B (2013) Evolution of some observed climate extremes in the West African Sahel. Weather Clim Extrem 1:19-25.

Moy AD, Howard WR, Bray SG, Trull TW (2009) Reduced calcification in modern Southern Ocean planktonic foraminifera. Nat Geosci 2(4):276-280.

Myneni RB, Keeling CD, Tucker CJ, et al (1997) Increased plant growth in the northern high latitudes from 1981 to 1991. Nature 386:698-702.

National Research Council (2014) Reducing Coastal Risk on the East and Gulf Coasts. National Academies Press, Wahington, DC, USA.

Ndebele-Murisa MR, Mashonjowa E, Hill T (2011) The implications of a changing climate on the Kapenta fish stocks of Lake Kariba. Trans R Soc South Africa 66:105-119.

New M, Hulme M, Jones P (2000) Representing twentieth-century space-time climate variability. Part II: Development of 1901-96 monthly grids of terrestrial surface climate. J Clim 13:22172238.

Niang I, Ruppel OC, Abdrabo MA, et al (2014) Africa. In: Barros VR, Field CB, Dokken DJ, et al (eds) Climate Change 2014: Impacts, Adaptation, and Vulnerability. Part B: Regional Aspects. Contribution of Working Group II to the Fifth Assessment Report of the Intergovernmental Panel on Climate Change. Cambridge University Press, Cambridge, UK and New York, NY, USA, pp 1199-1265.

Nicholls N (2011) Comments on "Influence of Location, Population, and Climate on Building Damage and Fatalities due to Australian Bushfire: 1925-2009." Weather Clim Soc 3:61-62.

Nicholls RJ, Woodroffe C, Burkett V (2009) Coastline degradation as an indicator of global change. In: Letcher TM (ed) Climate Change: Observed Impacts on Planet Earth. Elsevier, Oxford, UK, pp 409-424

Nichols T, Berkes F, Jolly D, et al (2004) Climate change and sea ice: Local observations from the Canadian Western Arctic. Arctic 57:68-79.

Nielsen J $\varnothing$, Sejersen F (2012) Earth System Science, the IPCC and the problem of downward causation in human geographies of Global Climate Change. Danish J Geogr 112:194-202.

NOAA (2014) Atlantic Hurricane Database (HURDAT2) 1851-2013. United States National Oceanographic and Atmospheric Administration. <http://www.nhc.noaa.gov/data\#hurdat> [Accessed May 27, 2014].

O'Loughlin J, Linke AM, Witmer FDW (2014a) Effects of temperature and precipitation variability on the risk of violence in sub-Saharan Africa, 1980-2012. Proc Natl Acad Sci USA 111:1671216717.

O'Loughlin J, Linke AM, Witmer FDW (2014b) Modeling and data choices sway conclusions about climate-conflict links. Proc Natl Acad Sci USA 111:2054-5.

O'Neill BC, Kriegler E, Riahi K, et al (2013) A new scenario framework for climate change research: the concept of shared socioeconomic pathways. Clim Change 122:387-400.

O'Neill SJ, Handmer J (2012) Responding to bushfire risk: the need for transformative adaptation. Environ Res Lett 7:014018. 
Oliver TH, Morecroft MD (2014) Interactions between climate change and land use change on biodiversity: attribution problems, risks, and opportunities. Wiley Interdiscip Rev Clim Chang 5:317-335.

Omumbo JA, Lyon B, Waweru SM, et al (2011) Raised temperatures over the Kericho tea estates: revising the climate in the East African highlands malaria debate. Malar J 10(1):12.

Oppenheimer M, Campos M, Warren R, et al (2014) Emergent risks and key vulnerabilities. In: Field CB, Barros VR, Dokken DJ, et al (eds) Climate Change 2014: Impacts, Adaptation, and Vulnerability. Part A: Global and Sectoral Aspects. Contribution of Working Group II to the Fifth Assessment Report of the Intergovernmental Panel on Climate Change Cambridge University Press, Cambridge, UK and New York, NY, USA, pp 1039-1099.

Oudin Åström D, Forsberg B, Ebi KL, Rocklöv J (2013) Attributing mortality from extreme temperatures to climate change in Stockholm, Sweden. Nat Clim Chang 3:1050-1054.

Parmesan C, Burrows MT, Duarte CM, et al (2013) Beyond climate change attribution in conservation and ecological research. Ecol Lett 16:58-71.

Parmesan C, Duarte C, Poloczanska E, et al (2011) Overstretching attribution. Nat Clim Chang 1:2-4.

Parmesan C, Yohe G (2003) A globally coherent fingerprint of climate change impacts across natural systems. Nature 421:37-42.

Pausas JG, Fernández-Muñoz S (2011) Fire regime changes in the Western Mediterranean Basin: from fuel-limited to drought-driven fire regime. Clim Change 110:215-226.

Peng C, Ma Z, Lei X, et al (2011) A drought-induced pervasive increase in tree mortality across Canada's boreal forests. Nat Clim Chang 1:467-471.

Peterson TC, Heim Jr. RR, Hirsch R, et al (2013a) Monitoring and Understanding Changes in Heat Waves, Cold Waves, Floods, and Droughts in the United States: State of Knowledge. Bull Am Meteorol Soc 94:821-834.

Peterson TC, Hoerling MP, Stott PA, Herring S (Eds) (2013b) Explaining extreme events of 2012 from a climate perspective. Bull Am Meteorol Soc 94:S1-S74.

Peterson TC, Stott PA, Herring S (Eds) (2012) Explaining extreme events of 2011 from a climate perspective. Bull Am Meteorol Soc 93:1041-1067.

Piontek F, Müller C, Pugh TAM, et al (2014) Multisectoral climate impact hotspots in a warming world. Proc Natl Acad Sci USA 111:3233-8.

Poloczanska ES, Brown CJ, Sydeman WJ, et al (2013) Global imprint of climate change on marine life. Nat Clim Chang 3:919-925.

Poloczanska ESS, Hoegh-Guldberg O, Cheung W, et al (2014) Cross-chapter box on observed global responses of marine biogeography, abundance, and phenology to climate change. In: Field CB, Barros, VB, Dokken DJ, et al (eds) Climate Change 2014: Impacts, Adaptation, and Vulnerability. Part A: Global and Sectoral Aspects. Contribution of Working Group II to the Fifth Assessment Report of the Intergovernmental Panel on Climate Change, Cambridge University Press, Cambridge, UK and New York, NY, USA, pp 123-127.

Pörtner H-O, Karl D, Boyd PW, et al (2014) Ocean systems. In: Field CB, Barros VR, Dokken DJ, et al (eds) Climate Change 2014: Impacts, Adaptation, and Vulnerability. Part A: Global and Sectoral Aspects.Contribution of Working Group II to the Fifth Assessment Report of the Intergovernmental Panel on Climate Change, Cambridge University Press, Cambridge, UK and New York, NY, USA, pp 411-484.

Post E, Forchhammer MC, Bret-Harte MS, et al (2009) Ecological Dynamics Across the Arctic Associated with Recent Climate Change. Science 325:1355-1358.

Powers LA, Johnson TC, Werne JP, et al (2011) Organic geochemical records of environmental variability in Lake Malawi during the last 700 years, Part I: the TEX86 temperature record. Palaeogeogr Palaeoclimatol Palaeoecol 303:133-139. 
Price RM, Bonnett D. (2000) Estimating the ratio of two Poisson means. Comput Stat Data Anal 34:345-356.

Pryzborowki J, Wilenski H (1940) Homogeneity of results in testing samples from Poisson series: With an application to testing clover seed for dodder. Biometrika 31:313-323.

Rahmstorf S, Coumou D (2011) Increase of extreme events in a warming world. Proc Natl Acad Sci USA 108:17905-17909.

Ranger N, Reeder T, Lowe J (2013) Addressing "deep" uncertainty over long-term climate in major infrastructure projects: four innovations of the Thames Estuary 2100 Project. EURO J Decis Process 1:233-262.

Rayner NA, Parker DE, Horton EB, et al (2003) Global analyses of sea surface temperature, sea ice, and night marine air temperature since the late nineteenth century. J Geophys Res 108(D14):4407.

Reisinger A, Kitching RL, Chiew F, et al (2014) Australasia. In: Barros VR, Field CB, Dokken DJ, et al (eds) Climate Change 2014: Impacts, Adaptation, and Vulnerability. Part B: Regional Aspects. Contribution of Working Group II to the Fifth Assessment Report of the Intergovernmental Panel on Climate Change. Cambridge University Press, Cambridge, UK and New York, NY, USA, pp 1371-1438.

Richardson AJ, Brown CJ, Brander K, et al (2012) Climate change and marine life. Biol Lett 8:907-909.

Rivera JA, Penalba OC, Betolli ML (2013) Inter-annual and inter-decadal variability of dry days in Argentina. Int J Climatol 33:834-842.

Root TL, MacMynowski DP, Mastrandrea MD, Schneider SH (2005) Human-modified temperatures induce species changes: Joint attribution. Proc Natl Acad Sci USA 102:7465-9.

Root TL, Price JT, Hall KR, et al (2003) Fingerprints of global warming on wild animals and plants. Nature 421:57-60.

Rosenzweig C, Casassa G, Karoly DJ, et al (2007) Assessment of observed changes and responses in natural and managed systems. In: Parry ML, Canziani OF, Palutikof JP, et al (eds) Climate Change 2007: Impacts, Adaptation and Vulnerability. Contribution of Working Group II to the Fourth Assessment Report of the Intergovernmental Panel on Climate Change. Cambridge University Press, Cambridge, UK, pp 79-131.

Rosenzweig C, Elliott J, Deryng D, et al (2014) Assessing agricultural risks of climate change in the 21st century in a global gridded crop model intercomparison. Proc Natl Acad Sci USA 111:326873.

Rosenzweig C, Karoly D, Vicarelli M, et al (2008) Attributing physical and biological impacts to anthropogenic climate change. Nature 453:353-357.

Rosenzweig C, Neofotis P (2013) Detection and attribution of anthropogenic climate change impacts. Wiley Interdiscip Rev Clim Chang 4:121-150.

Ruelland D, Tribotte A, Puech C, Dieulin C (2011) Comparison of methods for LUCC monitoring over 50 years from aerial photographs and satellite images in a Sahelian catchment. Int J Remote Sens 32:1747-1777.

Santer B, Wigley T, Barnett T, Anyamba E (1996) Detection of climate change and attribution of causes. In: Houghton JT, Meira Filho, LG, Callander BA, et al (eds) Climate Change 1995: The Science of Climate Change. Contribution of Working Group I to the Second Assessment Report of the Intergovernmental Panel on Climate Change. Cambridge University Press, Cambridge, UK, pp 407-443.

Schellnhuber HJ, Frieler K, Kabat $P$ (2014) The elephant, the blind, and the intersectoral intercomparison of climate impacts. Proc Natl Acad Sci USA 111:3225-7.

Schewe J, Heinke J, Gerten D, et al (2014) Multimodel assessment of water scarcity under climate change. Proc Natl Acad Sci U S A 111:3245-50. 
Schlenker W, Lobell DB (2010) Robust negative impacts of climate change on African agriculture. Environ Res Lett 5:14010.

Schlenker W, Roberts MJ (2009) Nonlinear temperature effects indicate severe damages to U.S. crop yields under climate change. Proc Natl Acad Sci USA 106:15594-15598.

Schneider U, Becker A, Finger P, et al (2014) GPCC's new land surface precipitation climatology based on quality-controlled in situ data and its role in quantifying the global water cycle. Theor Appl Climatol 115:15-40.

Seneviratne SI, Nicholls N, Easterling D, et al (2012) Changes in climate extremes and their impacts on the natural physical environment. In: Field CB, Barros V, Stocker TF, et al (eds) Managing the Risk of Extreme Events and Disasters to Advance Climate Change Adaptation. A Special Report of Working Groups I and II of the Intergovernmental Panel on Climate Change (IPCC). Cambridge University Press, Cambridge, UK and New York, NY, USA, pp 109-230.

Settele J, Scholes R, Betts R, et al (2014) Terrestrial and inland water systems. In: Field CB, Barros VR, Dokken DJ, et al (eds) Climate Change 2014: Impacts, Adaptation, and Vulnerability. Part A: Global and Sectoral Aspects. Contribution of Working Group II to the Fifth Assessment Report of the Intergovernmental Panel on Climate Change. Cambridge University Press, Cambridge, UK and New York, NY, USA, pp 271-359.

Shiogama $\mathrm{H}$, Stone DA, Nagashima $\mathrm{T}$, et al (2012) On the linear additivity of climate forcing-response relationships at global and continental scales. Int J Climatol 33:2542-2550.

Sitoki L, Gichuki J, Ezekiel C, et al (2010) The Environment of Lake Victoria (East Africa): Current Status and Historical Changes. Int Rev Hydrobiol 95:209-223.

Smith JB, Schellnhuber J, Mirza M, et al (2001) Vulnerability to climate change and reasons for concern: A synthesis. In: McCarthy JJ, Canziani OF, Leary NA, et al (eds) Climate change 2001: Impacts, Adaptation and Vulnerability. Contribution of Working Group II to the third assessment report of the Intergovernmental Panel on Climate Change. Cambridge University Press, Cambridge, UK, pp 913-967.

Smith JB, Schneider SH, Oppenheimer M, et al (2009) Assessing dangerous climate change through an update of the Intergovernmental Panel on Climate Change (IPCC) "Reasons for concern." Proc Natl Acad Sci USA 106:4133-4137.

Smith KRR, Woodward A, Campbell-Lendrum D, et al (2014) Human health: impacts, adaptation, and co-benefits. In: Field CB, Barros VR, Dokken DJ, et al (eds) Climate Change 2014: Impacts, Adaptation, and Vulnerability. Part A: Global and Sectoral Aspects. Contribution of Working Group II to the Fifth Assessment Report of the Intergovernmental Panel on Climate Change. Cambridge University Press, Cambridge, UK and New York, NY, USA, pp 709-754.

Solow AR (2013) A call for peace on climate and conflict. Nature 497:179-180.

Stager JC, Hecky RE, Grzesik D, et al (2009) Diatom evidence for the timing and causes of eutrophication in Lake Victoria, East Africa. Hydrobiologia 636:463-478.

Stammer DA, Cazenave A, Ponte RM, Tamisiea ME (2013) Causes for contemporary regional sea level changes. Ann Rev Mar Sci 5:21-46.

Stanley T (2001) Wheat From Chaff: Meta-Analysis As Quantitative Literature Review. J Econ Perspect 15:131-150.

Steffen W, Grinevald J, Crutzen P, McNeill J (2011) The Anthropocene: conceptual and historical perspectives. Philos Trans A Math Phys Eng Sci 369:842-67.

Stocker TF, Plattner GK (2014) Rethink IPCC reports. Nature 513:163-165.

Stocker TF, Qin D, Plattner GK, et al (2011) Tried and tested. Nat Clim Chang 1:71.

Stone DA, Auffhammer M, Carey M, et al (2013) The challenge to detect and attribute effects of climate change on human and natural systems. Clim Change 121:381-395. 
Stone DA, Allen MR (2005) The end-to-end attribution problem: from emissions to impacts. Clim Change 71:303-318.

Stone DA, Allen MR, Stott PA, et al (2009) The detection and attribution of human influence on climate. Annu Rev Env Resour 34:1-16.

Stott PA, Allen M, Christidis N, et al (2013) Attribution of weather and climate-related extreme events. In: Climate Science for Serving Society. Springer, Netherlands, pp 307-337.

Stott PA, Gillett NP, Hegerl GC, et al (2010) Detection and attribution of climate change: a regional perspective. Wiley Interdiscip Rev Clim Chang 1:192-211.

Stott PA, Stone DA, Allen MR (2004) Human contribution to the European heatwave of 2003. Nature 432:610-614.

Syvitski JPM, Kettner AJ, Overeem I, et al (2009) Sinking deltas due to human activities. Nat Geosci 2:681-686.

Tao F, Yokozawa M, Xu Y, et al (2006) Climate changes and trends in phenology and yields of field crops in China, 1981-2000. Agric For Meteorol 138:82-92.

Tao F, Zhang Z, Zhang S, et al (2012) Response of crop yields to climate trends since 1980 in China. Clim Res 54:233-247.

Taylor KE, Stouffer RJ, Meehl GA (2012) An overview of CMIP5 and the experiment design. Bull Am Meteorol Soc 93:485-498.

Thomas DSG, Twyman C, Osbahr H, Hewitson B (2007) Adaptation to climate change and variability: farmer responses to intra-seasonal precipitation trends in South Africa. Clim Change 83:301322.

Thompson SL, Schneider SH (1982) Carbon dioxide and climate: Has a signal been observed yet? Nature 292:645-646.

Tierney JE, Mayes MT, Meyer N, et al (2010) Late-twentieth-century warming in Lake Tanganyika unprecedented since AD 500. Nat Geosci 3:422-425.

Trenberth KE (2012) Framing the way to relate climate extremes to climate change. Clim Change 115:283-290.

Trewin B, Vermont $\mathrm{H}$ (2010) Changes in the frequency of record temperatures in Australia, 19572009. Aust Meteorol Oceanogr J 60:113-119.

Van Vuuren DP, Isaac M, Kundzewicz ZW, et al (2011) The use of scenarios as the basis for combined assessment of climate change mitigation and adaptation. Glob Environ Chang 21:575-591.

Vargas WM, Naumann G, Minetti JL (2010) Dry spells in the River Plata Basin: an approximation of the diagnosis of droughts using daily data. Theor Appl Climatol 104:159-173.

Vaughan DG, Comiso JC, Allison I, et al (2013) Observations: Cryosphere. In: Stocker TF, Qin D, Plattner GK, et al (eds) Climate Change 2013: The Physical Science Basis. Contribution of Working Group I to the Fifth Assessment Report of the Intergovernmental Panel on Climate Change. Cambridge University Press, Cambridge, UK and New York, NY, USA, pp 317-382.

Verburg P, Hecky RE (2009) The physics of the warming of Lake Tanganyika by climate change. Limnol Oceanogr 54:2418-2430.

Verheyen R (2012) Tackling Loss \& Damage - A new role for the climate regime? The loss and damage in vulnerable countries initiative. <www.lossanddamage.net/download/6877.pdf>.

Víctor H. Gutiérrez-Vélez, Uriarte M, DeFries R, et al (2014) Land cover change interacts with drought severity to change fire regimes in Western Amazonia. Ecol Appl 24:1323-1340.

Vincke C, Diédhiou I, Grouzis M (2010) Long term dynamics and structure of woody vegetation in the Ferlo (Senegal). J Arid Environ 74:268-276.

Walter SD (1976) The estimation and interpretation of attributable risk in health research. Biometrics 32:829-849. 
Warszawski L, Frieler K, Huber V, et al (2014) The Inter-Sectoral Impact Model Intercomparison Project (ISI-MIP): project framework. Proc Natl Acad Sci USA 111:3228-32.

Wassmann P, Duarte CM, Agustí S, Sejr MK (2011) Footprints of climate change in the Arctic marine ecosystem. Glob Chang Biol 17:1235-1249.

Weatherhead E, Gearheard S, Barry RG (2010) Changes in weather persistence: Insight from Inuit knowledge. Glob Environ Chang Policy Dimens 20:523-528.

Webb LB, Whetton PH, Bhend J, et al (2012) Earlier wine-grape ripening driven by climatic warming and drying and management practices. Nat Clim Chang 2:259-264.

Webster PJ, Holland GJ, Curry JA, Chang HR (2005) Changes in tropical cyclone number, duration, and intensity in a warming environment. Science 309:1844-1846.

Weed M (2005) Meta Interpretation: A Method for the Interpretive Synthesis of Qualitative Research. Forum Qual Sozialforsch/Forum Qual Soc Res 6:1-14.

Westerling AL, Hidalgo HG, Cayan DR, Swetnam TW (2006) Warming and earlier spring increase western U.S. forest wildfire activity. Science 313:940-3.

Wezel A, Lykke AM (2006) Woody vegetation change in Sahelian West Africa: Evidence from local knowledge. Environ Dev Sustain 8:553-567.

WGMS (1989) World Glacier Inventory - Status 1988. Haeberli W, Bösch H, Scherler K et al (eds). IAHS(ICSI), UNEP, UNESCO.

Whittaker J, Haynes K, Handmer J, McLennan J (2013) Community safety during the 2009 Australian "Black Saturday" bushfires: an analysis of household preparedness and response. Int J Wildl Fire 22:841.

Wigley TML, Barnett TP, et al (1990) Detection of the greenhouse effect in the observations. In: Houghton JT, Jenkins GJ, Ephraums JJ (eds) Climate Change. The IPCC Scientific Assessment. Cambridge University Press, Cambridge, UK, pp 241-255.

Williams PA, Allen CD, Macalady AK, et al (2012) Temperature as a potent driver of regional forest drought stress and tree mortality. Nat Clim Chang 3:292-297.

Williams RJ, Bradstock RA, Cary GJ, et al (2009) Interactions between climate change, fire regimes and biodiversity in Australia - a preliminary assessment. Report to the Department of Climate Change and Department of the Environment, Heritage and Arts, Canberra, Australia.

Wilson E (1927) Probable inference, the law of succession, and statistical inference. J Am Stat Assoc 22:209-212.

Wong PP, Losada IJ, Gattuso J-P, et al (2014) Coastal systems and low-lying areas. In: Field CB, Barros VR, Dokken DJ, et al (eds) Climate Change 2014: Impacts, Adaptation, and Vulnerability. Part A: Global and Sectoral Aspects. Contribution of Working Group II to the Fifth Assessment Report of the Intergovernmental Panel on Climate Change. Cambridge University Press, Cambridge, UK and New York, NY, USA, pp 361-409.

Wood SA, Jina AS, Jain M, et al (2014) Smallholder farmer cropping decisions related to climate variability across multiple regions. Glob Environ Chang 25:163-172.

Woodworth PL, Menéndez M, Roland Gehrels W (2011) Evidence for century-timescale acceleration in mean sea levels and for recent changes in extreme sea levels. Surv Geophys 32:603-618.

Zak MR, Cabido M, Cáceres D, Díaz S (2008) What drives accelerated land cover change in central Argentina? Synergistic consequences of climatic, socioeconomic, and technological factors. Environ Manage 42:181-9.

Zwiers F, Hegerl G (2008) Climate change: attributing cause and effect. Nat Reports Clim Chang 453:296-297. 


\section{Summary}

Global anthropogenic climate change is unequivocal. This thesis addresses the question whether and to what extent the impacts of anthropogenic climate change are already observed, i.e. detected and attributed, in natural and human systems.

My research is rooted in analysis and expert elicitation performed during the IPCC's working group II fifth assessment cycle. It carries forward questions that remained open after the completion of IPCC's Fifth Assessment Report, and narrows gaps left by the lack of integration between the WGI and WGII assessments. For one, the widespread perception that anthropogenic climate change is already affecting a number of vulnerable human and managed systems, is contrasted by the relative lack of documented evidence of observed climate-change impacts for those vulnerable systems reported in IPCC'S AR5. This inconsistency is exacerbated by the failure of IPCC'S AR5 to assess the link between the impacts caused by regional climate trends and the contribution of anthropogenic forcing to these climate trends. Addressing these issues and clarifying some of the underlying controversies and misperceptions regarding the concept and requirements of impact detection and attribution was a major motivation for this thesis.

Detection and attribution exercises address the question whether something has changed and examine the causes of that change. The endpoint of an attribution exercise in the context of impact will often be a change in a climate variable as opposed to other drivers of change, such as land-use or pollution, while detection and attribution in the context of climate science focusses on distinguishing the role of anthropogenic forcing from that of other external acts of forcing and natural variability.

Existing concepts of detection and attribution mostly apply to the disciplinary analysis of wellbounded systems, i.e. the global climate system, and therefore need to be adapted to be suitable in the interdisciplinary and multifactorial context of most impact systems. For example, the effect of a sustained trend in a climate variable may be masked, compounded, overcompensated or amplified by the effect of other drivers, including unconscious or explicit adaptation to observed or expected climate change. Also, establishing causal relations is very challenging in multifactorial environments and more so within the field of qualitative social research, where quantitative data may be absent or limited to a very narrow set of case studies.

Chapter 2 develops a five-step guidance to impact attribution that considers all the necessary links in the causal chain from observed changes in impact systems to observed changes in climate, 
elaborating on the concept of 'impact detection' established during the AR5. It then applies those steps to five examples of observed impacts in human and managed systems from the literature, illustrating the way that multiple lines of evidence can be integrated into an overall assessment of confidence in impact attribution. Still, impact detection and attribution analysis sets a very high bar in terms of system understanding and data requirements. Historical monitoring is inadequate for many systems that are sensitive to climate, especially in developing countries. However, the imbalance in the documentation of observed impacts across the globe could be alleviated if the protocols outlined in Chapter 2 were applied in a consistent manner using available data.

A central challenge that is outside of the scope of Chapters 2's framework consists in the attribution of impacts of extreme weather, or rather the lack thereof. Adverse effects related to the climate most clearly manifest through periods of extreme weather, such as severe storms or heat waves. However, to detect and attribute changes in climate variability is more difficult than just changes in means. Understanding both the statistical foundation of single event attribution and the interplay of climate hazard, exposure and vulnerability for the severity of the actual impact event are crucial in order to explain the role of a changing climate in loss and damage experienced during periods of extreme weather. In particular for very rare events, single event attribution examining the role of anthropogenic forcing in the occurrence and intensity of specific events is increasingly being carried out by scientists. Chapter 3 uses a simple statistical model of the occurrence of events to elucidate some fundamental issues in single-event attribution. It shows that single-event attribution is simply a re-casting of the attribution of a change in the overall rate of events. Confidence intervals are constructed based on a Poisson process model representing pre- and post-climate change situations. Results show that confident attribution of a single event generally requires a very large change in the overall rate. If detection and attribution analysis shall provide a tool for systematic assessment of observed impacts, capturing the contribution of anthropogenic forcing to impacts of extreme weather beyond the probabilistic attribution of hazard risk is an important research need.

The direct attribution of observed impacts to anthropogenic forcing is deemed very difficult and rarely done. Therefore, the influence of anthropogenic forcing for observed impacts of climate change cannot be assessed based on the available scientific literature. In contrast, impacts of recent changes in regional climate on natural and human systems have been observed and documented globally, though the available evidence is unevenly distributed. Gaps persist especially in tropical regions, and for many human and managed systems. Chapter 4 provides a comprehensive assessment of the evolution of the evidence base for observed climate change impacts across three major systems (physical, biological and human) and eight world regions by analysing and 
summarizing the results of IPCC's third, fourth and fifth assessment reports regarding the number of impacted subcategories and the quantity and spatial extent of evidence.

Such evidence has increased substantially over the last decades and is now available across systems and world regions. A large fraction of the most robust evidence for the effects of recent climate change is related to the cryosphere and to ecosystems that are highly sensitive to temperature (e.g. alpine ecosystems). Many of these observations also served as early indications of global warming. Extensive impacts, i.e. where climate change has become a dominant driver of pervasive impacts across large regions, are documented for shifts in species ranges and phenologies in both marine and terrestrial ecosystems, large scale vegetation changes in the Arctic tundra, increased mass bleaching and mortality in tropical reef-building corals, enhanced glacier melt, mountain and lowland permafrost thaw, and changes in patterns of river run-off related to glacier melt, permafrost thaw and changes in seasonal snow accumulation. Evidence has also substantiated for other groups of impacts, most notably for impacts of climate change on wildfire regimes, crop yields and the livelihoods of Arctic indigenous peoples. Still, the confident detection and attribution of climate change impacts is particularly challenging in many human and managed systems and in areas with poor monitoring records. Innovate methods have been explored to fill in such gaps through, for instance, analysis of archival footage or consulting local and indigenous knowledge.

The uneven global distribution of knowledge about observed impacts also highlights several challenges involved in conveying complex scientific information in a policy context, as discussed in Chapter 6. Most prominently, the absence of evidence in some areas should not be interpreted as evidence for the absence of impacts. Complementary information on current vulnerability and observed and expected climate trends would be helpful to place detection and attribution findings into the wider science-policy perspective.

Finally, to assess the role of anthropogenic forcing in the impacts attributed to recent climate change, I apply a new method of 'systematic climate change detection and attribution' to the regional climate trends corresponding to the set of impacts reported in AR5. This method evaluates both the quality and adequacy of observational climate data, and the consistency of observed climate trends with model results representing the historic climate and a hypothetical natural climate without the effect of anthropogenic forcing for the same period and region.

Results of my analysis in Chapter 5 indicate that anthropogenic forcing is implicated with a major role in the majority of temperature trends related to the observed impacts, whereas the link between precipitation changes and human influence on the climate remains uncertain. As a result of a multi-step attribution assessment that combines the respective impact attribution and climate- 
change attribution steps, approximately three-quarters of the aforementioned extensive impacts are attributed to anthropogenic forcing, with at least medium confidence in a major role. Overall, 66 of the 118 impacts analysed (i.e. 56\%) are attributed to anthropogenic forcing of the climate system with at least medium confidence in a major or minor role; however, none of these is mediated by precipitation. Limiting the analysis to effects of warming consequently increases the corresponding share of attributed effects to approximately $65 \%$ and this number rises to almost three-quarters if detection is considered instead of attribution. On average, confidence in climate-change attribution is considerably higher than confidence in impact attribution, indicating a gap in research concerning the evaluation of climate-change effects in human and natural systems. Despite the high share of impacts that can be linked to anthropogenic climate change, no direct relationship can be inferred between confidence in impact attribution and confidence in climate-change attribution.

Impacts on the livelihoods of Arctic indigenous peoples are the only incidence where anthropogenic climate change is assessed to have a major role in an impact observed in a human system. However, for another $30 \%$ of temperature related observed effects in human and managed systems, at least a minor role of anthropogenic climate change is attributed with medium confidence. The comparatively minor role of recent climate change in most impacts in human and managed systems and the relatively low confidence in those observations are the limiting factors in their attribution to anthropogenic forcing. A focus on robust impact attribution as presented in Chapter 5 therefore risks to side line emerging climate-change impacts prevalent especially in human systems.

My research augments the understanding of observed changes by clarifying concepts and definitions relating to the attribution of impacts to climate change and extreme weather events, and by providing an analysis of the role of anthropogenic forcing for documented climate change impacts in natural and human systems. The scope and nature of available observations strongly influences the possible outcome of any detection and attribution assessment. The more consistent treatment of 'white spots' in our knowledge and the communication of the reasons for those spots would help to clarify the relevance of detection and attribution results for policymakers.

The overarching goal of this thesis is to elucidate to what extent the impacts of anthropogenic climate change can be detected and attributed worldwide, in particular within human and managed systems. This is achieved by assessing current impacts of anthropogenic climate change and by addressing the sources of uncertainty, assigning confidence levels and discussing the knowledge differences across a range of relevant climate variables, regions, systems and research disciplines. While the impact of anthropogenic climate change on natural and - to a lesser degree - human systems is confirmed by my analysis, its extent and magnitude cannot be summarized across all climate variables and sectors. 


\title{
Annex A
}

\section{Rapid systematic assessment of the detection and attribution of regional anthropogenic climate change}

\author{
Systematic detection and attribution
}

Dáithí A. Stone, Gerrit Hansen

Based on a manuscript submitted to Climate Dynamics

\section{The confidence estimation algorithm}

This Annex elaborates in detail on the rapid systematic assessment of the detection and attribution of regional anthropogenic climate change applied in Chapter 5.

\subsection{The confidence metric}

The desired output of this attribution assessment is a description of confidence concerning the attribution of a major role of anthropogenic emissions in an observed change in regional climate. The algorithm is centred at the comparison of observed variations in the climate against our expectations of how the climate should have changed, with the latter developed through some sort of understanding of how the climate might respond to external drivers (Hegerl et al 2010; Hegerl and Zwiers 2011). Around that comparison we must also consider the adequacy of our understanding, of the implementation of our understanding and of the inputs to the analysis.

Mastrandrea et al (2010) formulate two qualitative descriptors for use in assessments conducted by the Intergovernmental Panel on Climate Change for summarizing current understanding of various statements regarding climate change. The intention of the algorithm presented here is to estimate belief in whether a statement of attribution is accurate based on direct evidence, so we adopt the confidence descriptor here. This confidence descriptor merges evaluation of the quality and quantity of evidence (Section 1.3) and of the degree of agreement across sources of evidence (Section 1.4, 1.5) into a single qualitative assessment. Such confidence descriptors have been adopted in the detection and attribution chapters of all recent IPCC assessment reports (Seneviratne et al 2012; Bindoff et al 2013; Cramer et al 2014), allowing direct comparison in Section 2 between results estimated here and in two of those reports.

While the output may be qualitative, the algorithm itself is quantitative; the mapping from the latter to the former is described in Section 1.6. It uses a numerical metric $c$ to characterize confidence (Figure 1). The metric is assigned an initial value which depends on the number of input data sources. This value is then left unchanged or reduced based on a series of tests which examine our belief in the adequacy of the input data sources and our understanding of the relevant processes (Section 1.3). It is then further left unchanged or modified based on a series of tests which evaluate the agreement between observed changes and our expectations based on process-based modelling (Section 1.5). All of these tests output a confidence-reduction factor $y$ by which the confidence 
metric is multiplied. Because reduction of the metric is always by multiplication, the order of the tests does not in fact matter.

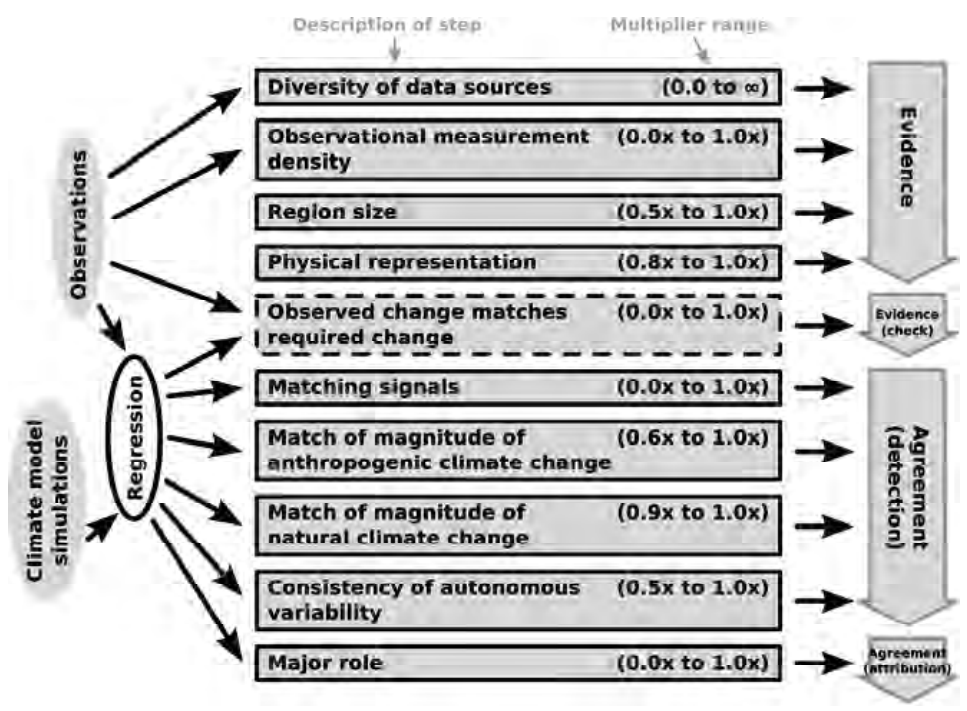

Figure 1: Schematic of the algorithm for estimating the confidence in the detection and attribution of a climate response to anthropogenic drivers. An initial metric based on the availability of data products is degraded according to tests against various criteria. These tests are described in Sections 1.3 through 1.5.

\subsection{Ingredients}

The steps of the algorithm for estimating confidence for a given aspect of climate change use various combinations of the following inputs:

- Information about the climate change of interest, specifically identification of the climate variable, specification of the seasonal and of the spatial extent. A direction of change may also be specified (Section 1.4);

- $\quad \mathrm{N}_{\mathrm{obs}}$ gridded observational products. The usage of multiple data sets allows some (albeit likely incomplete) inclusion of measurement error and the uncertainty in calculating a regionally and seasonally averaged estimate from instantaneous point measurements;

- Simulations of the climate system from $\mathrm{N}_{\text {mod }}$ models which have been driven with all known possible important drivers of climate change, including both anthropogenic and natural drivers. For dynamical climate models, averaging across multiple simulations which produce different possible weather trajectories for each model provides a more accurate estimate of the model's response signal;

- Simulations of the climate system from the same $\mathrm{N}_{\bmod }$ climate models but which have been driven with natural drivers only; and

- A large number of years of simulations of dynamical climate models with no variations in external drivers beyond the annual cycle. While in practice it would be preferable to have these for each of the above $\mathrm{N}_{\text {mod }}$ climate models, in practice the sampling requirement of a large ensemble dictates that we will assume that in general data will be borrowed from available simulations of these and other models. 


\subsection{Assessment of data sources (evidence)}

\section{Diversity of data sources}

The observational and (after-the-fact) prediction products comprise our ultimate sources of evidence, so having more numerous independent products available should increase the robustness of the evidence base. We consider the predictions of response signals to come solely from simulations of dynamical climate models (e.g. Taylor et al 2012), generally of about the same generation. Note however that having models of different generations, for instance "state-of-theart" dynamical models and "back-of-the-envelope" zero-dimensional models, could add substantially to confidence. If the errors in the variability and response signals of climate models were independent, then we would expect the information content to scale as $\sqrt{N_{\text {mod }}}$. However, because climate models have not been developed in isolation, it might be expected that they share errors. Indeed recent studies suggest that ad hoc collections of climate models of similar levels of complexity may have an effective sample size only about one half the total number of models (Jun et al 2008; Pennell and Reichler 2010) in terms of the errors in their mean climatology. In the absence more relevant studies, we assume that a similar property holds for inter-annual variability.

Similarly, observational data products are not independent of each other, most particularly in sharing most of the measurements they use as input. Studies of the effective sample size of observational products are currently lacking, but in any case would likely depend on variable and region. Through analogy, we suppose a similar scaling as for climate models. In research-grade assessments, it may be possible to better characterize these effective sample sizes, for instance noting the degree with which the observational products share the input data. The important assumption here is that the information content scales as $\sqrt{N}$. The mapping of the metric to the qualitative levels described in Section 1.6 includes a calibration that would override any multiplicative constant added here, so we take the initial confidence as

$$
\gamma=\sqrt{\mathrm{Nmod} \cdot \mathrm{Nobs}}
$$

While in theory this allows an infinite initial confidence, in practice only reasonable values of $\gamma_{\text {sources }}$ are possible with the current diversity of data sources (see Section 2.1).

\section{Observational measurement density}

The spatial distribution of observational measurements is not uniform, and in some areas may be considered low enough to prevent the development of confidence in their representativeness of the regional climate. To estimate the adequacy of measurement density, we estimate the fraction of the variance of the time series of a regional climate variable that is accounted for by the given measurement density using a method building on that employed in New et al (2000). For the landbased variables, we consider the number of stations reporting in the month for each grid cell; sea surface temperature products instead report the total number of individual measurements taken from moving ships, so we divide by 5 to get an effective number of stations (Jones et al 1997).

The stations in each grid cell (or 'effective stations' in the case of ocean data) are assigned random locations within that grid cell, subject to land/sea definitions. Stations are considered to only become active or inactive in specific order (e.g. they do not move). Stations that are active for less 
than $90 \%$ of the total period examined are discarded. We then estimate the fraction of the variability accounted for by the available active stations at each point within the region on a higher resolution $\left(0.1^{\circ} \times 0.1^{\circ}\right.$ longitude-latitude $)$ grid.

Only stations within the decorrelation radius, $r_{\text {decorr }}$, of the grid cell are considered (New et al 2000). It is assumed that differences between two stations separated by distance $r_{\text {stat }}$ can be represented as random noise that is correlated in space with fractional variance $1-e^{-2 \cdot \frac{r s t a t}{r d e c o r r}}$.

The fractional variance accounted for by the station coverage, $Y_{\text {density }}$, is then provided by the integral on the high-resolution grid of the product of the variance unaccounted for by the given stations:

$$
\gamma_{\text {density }}=\frac{1}{N \cdot a_{\text {region }}}\left(\sum_{\text {year }} \sum_{\text {cell }}\left(a_{\text {cell }} \cdot\left(1-\pi_{\text {stat }(\text { cell,year })}\right) \cdot e^{-2 \frac{r_{\text {stat }}}{r_{\text {decorr }}}}\right)\right)
$$

where $a_{\text {cell }}$ and $a_{\text {region }}$ are the spatial area of the cell on the high-resolution grid and of the region respectively, $N_{y e a r}$ is the number of years in the period, and the stations being considered (stat(cell, year)) varies with location and year.

In practice, measurement density is not available for most observational products, so for the evaluation conducted in Section 2 we only obtain this information from one product for each climate variable (Table 1).

Table 1: Observational data products used to characterize observational monitoring density around the globe. The decorrelation radius is also given for each variable (New et al 2000).

\begin{tabular}{l|l|l} 
Climate variable & Station density data set & Decorrelation \\
\hline 2m air temperature & CRU TS 3.22 (Harris et al 2014) & $1200 \mathrm{~km}$ \\
Precipitation & CRU TS 3.22 (Harris et al 2014) & $450 \mathrm{~km}$ \\
Sea surface temperature & HadSST3.1.1.0 (Kennedy et al 2011a;b) & $1200 \mathrm{~km}$
\end{tabular}

For the land-based variables these observation counts are for a relatively high-spatial-resolution product, while for sea surface temperature the information is only available for the relatively coarse resolution $\left(200000 \mathrm{~km}^{2}\right.$ ) HadSST3.1.1.0. The HadSST3.1.1.0 density data, which consider in situ data only, will furthermore be an underestimate for higher resolution products that also use satellite data in more recent decades, such as those used in the analysis of Section 2; this could produce a bias toward lower confidence in regions with a small number of in situ measurements, such as the Antarctic Ocean.

Region size

Dynamical climate models have a limited spatial resolution, meaning they are better at reproducing variations in large-scale mechanisms than ones closer to the resolution size. Furthermore, the smaller scale characteristics of the anthropogenic drivers of climate change (particularly aerosol emissions) are less well understood than the larger scale properties. This means we are a priori less confident in analyses of climate model predictions for smaller regions. Similarly, the accuracy of 
observational products becomes more sensitive to the interpolation method used at scales around or smaller than the station separation. To account for this, the confidence metric is reduced by an amount related to the region's size. If $a_{\text {region }}$ is the area covered by the region in units of $10^{6} \mathrm{~km}^{2}$, then the confidence metric is multiplied by

$$
\gamma_{\text {size }}=0.5+\frac{\arctan \left(a_{\text {region }}\right)}{\pi}
$$

The functional form is such that $\gamma \sim 1$ at continental scales (Jones et al 2013), and such that $\gamma \sim$ 0.5 at scales around the smallest dynamical resolution of the current generation of climate models (about $4^{2}$ times larger than the grid cell size; Figure 2). The lower value of 0.5 is a balance between acknowledging that the modelling and observational products may retain some skill even if they are not fully resolving processes and features and realising that size-related inaccuracies are also likely to emerge as penalties in the tests described in Section 1.5 which we do not want to double count.
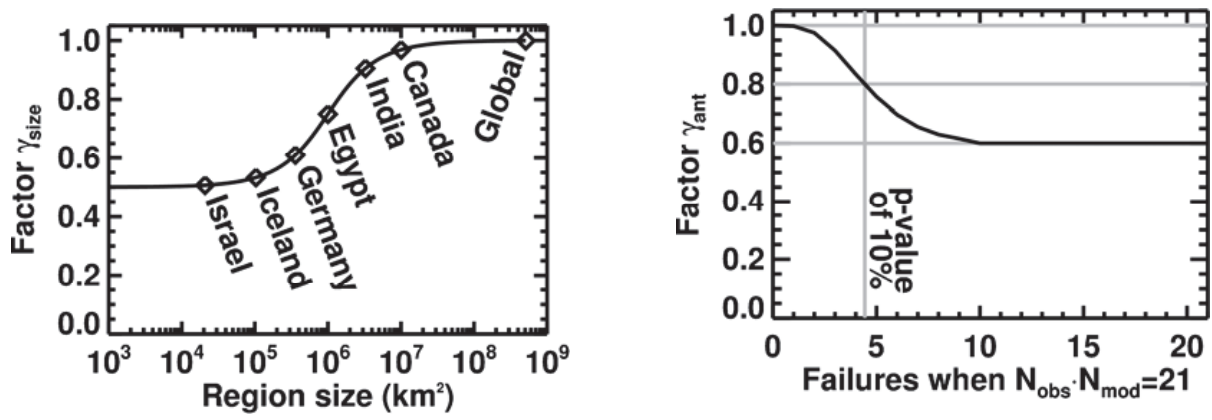

Figure 2: The functional forms of two of the confidence multipliers underlying the algorithm. Left: the multiplication factor relating to region size, with representative countries listed. Right: the multiplication factor relating to whether the predicted magnitude of the response to anthropogenic emissions matches the observed magnitude, as estimated by linear regression, for the case of $\mathbf{2 1}$ combinations of observational and model data products.

Physical respresentation

The basic physical processes behind some aspects of the climate are both well understood and mostly resolved in dynamical models, but this is not the case for some variables. For instance, the microphysical processes that generate precipitation are not simulated in climate models, but rather are approximated by somewhat heuristic algorithms. In recognition of this, the confidence metric is multiplied by a constant that depends only on the climate- variable:

$$
\gamma_{\text {physics }}=\left\{\begin{array}{c}
0.8 \text { for precipitation } \\
1 \text { for temperature }
\end{array}\right.
$$

The general effect on confidence for precipitation is a reduction of one of the Mastrandrea et al (2010) levels. 


\subsection{Observed change matches required change}

This is an optional test that applies only in cases where there is a requirement for the sign of the observed trend over the period. It is not applicable in the examples in Sections 2, but required for the implementation in chapter 5 of this thesis. In that case the impacts are reported to have been caused by a specific observed climate trend, and so a necessary step is to confirm that that trend also exists in the observational data sets used in the climate change attribution analysis. Reasons for discrepancies could include ambiguity over regional, period, or seasonal definitions, or disagreement between local and global data sets. If $\mathrm{N}_{\text {sign }}$ is the number of observational data sets producing trends which match the sign of the required trends, then the confidence metric is multiplied by

$$
\gamma_{\text {sign }}=\operatorname{plus}\left(2 \cdot \frac{N_{\text {sign }}}{N_{\text {obs }}}-1\right)
$$

where $\operatorname{plus}(x)=x$ if $x>0$, and otherwise equals 0 . Thus, $\gamma_{\text {sign }}=1$ if all of the observational data sets produce the required sign, but $\mathrm{y}_{\text {sign }}=0$ if half or fewer do so.

\subsection{Comparison between the data sources (agreement)}

\section{Comparison of observed and predicted climate change}

The analysis method behind much research into the detection and attribution of climate change in recent years applies a linear regression model to compare output from climate model simulations against observed climate changes (Bindoff et al 2013). The central idea is to separate aspects of the climate response that we consider to be known (i.e. tightly constrained by external parameters) from those that we consider to be less well known (i.e. not tightly constrained). The pattern by which the climate system is expected to respond to a particular external driver is generally considered robust and well estimated by past and current dynamical climate models; thus the pattern can act as a fingerprint for that response. For instance, both the climate system and models of the climate system should respond to a large volcanic eruption soon after that eruption, a feature that distinguishes its response from other drivers. In contrast, the magnitude of the response may not be something that is particularly well estimated by current climate models, because this depends on feedback processes within the climate system, such as how cloud microphysics interact with the larger scale climate, whose effects are not so directly constrained.

With this in mind, if $X_{o b s}(t)$ represents variations in an observed climate variable as a function of time $t, X_{\text {ant }}(t)$ represents the expected climate response to anthropogenic external drivers, and $X_{\text {nat }}(t)$ represents the expected climate response to natural external drivers, then the regression can be written as (Allen and Tett 1999):

$$
X_{\text {obs }}(t)=\beta_{\text {ant }} \cdot X_{\text {ant }}(t)+\beta_{\text {nat }} \cdot X_{\text {nat }}(t)+R(t)
$$

Here $R(t)$ is the residual of the regression and $\beta_{\text {ant }}$ and $\beta_{\text {nat }}$ are the regression coefficients estimated such that the variance of $R(t)$ is minimised. This formulation of the regression assumes that we 
can perfectly estimate the $X_{\text {ant }}(t)$ and $X_{\text {nat }}(t)$ responses (Allen and Stott 2003). In the test cases examined in Sections 2 this assumption is important because the estimates will be derived from a small number of climate model simulations. However, the resulting underestimate in the uncertainty from the regression will be compensated by repetitive estimation (and solving of the regression equation) using multiple ( $\mathrm{N}_{\text {obs }} \cdot \mathrm{N}_{\text {mod }}$ ) combinations of observational and climate model data sources.

The regression assumes that responses to climate change are linearly additive, which appears reasonable for temperature and precipitation responses of the magnitude and spatial scale considered here (Shiogama et al 2012). Available climate model data (Section 2.1) generally only cover the $\mathrm{X}_{\text {nat }}(\mathrm{t})$ response signal and the $\mathrm{X}_{\text {all }}(\mathrm{t})=\mathrm{X}_{\text {ant }}(\mathrm{t})+\mathrm{X}_{\text {nat }}(\mathrm{t})$ response to the full combination of anthropogenic and natural drivers. Substituting into Equation 6 we get:

$$
X_{\text {obs }}(t)=\beta_{\text {ant }} \cdot X_{\text {all }}(t)+\left(\beta_{\text {nat }}-\beta_{\text {ant }}\right) \cdot X_{\text {nat }}(t)+R(t)
$$

The regression coefficients $\beta_{\text {ant }}$ and $\beta_{\text {nat }}+\beta_{\text {ant }}$ and their uncertainty due to the limited sampling of the observed climate response against the noise of natural internally generated variability of the climate system are estimated using the code available at http://www.csag.uct.ac.za/ daithi/idl lib/detect/ (Allen and Tett 1999). Traditionally, a response to anthropogenic forcing is considered to be detected if $\beta_{\text {ant }}$ is positive and inconsistent with zero at some level of statistical significance given this sampling uncertainty.

The regression is performed separately for each combination of the $\mathrm{N}_{\mathrm{obs}}$ observation data products and the $\mathrm{N}_{\text {mod }}$ climate model products. The translation of these regression analyses into modification of the confidence metric is described in following sections.

\section{Matching signals}

This test addresses the question whether the fingerprint of the anthropogenic response expected by the climate models is indeed found in the observational data. In terms of the regression, the question is whether $\beta_{\text {ant }}>0$. This step is the critical test for a climate change detection analysis. If we suppose that each of the climate models and observational products represent random samples of the probability distributions of possible models and observations respectively, then we can add the probability distributions for each of the $\mathrm{N}_{\text {obs }} \cdot \mathrm{N}_{\text {mod }}$ observation-model combinations and calculate the fraction $f_{\text {signal }}$ of the combined distribution that is greater than zero. The confidence metric is then multiplied by $\mathrm{Y}_{\text {signal }}=\mathrm{f}_{\text {signal }}$.

Match of magnitude of anthropogenic climate change

Whether the magnitude of the observed signal matches the predicted magnitude is often considered a component of attribution rather than detection (Hegerl et al 2010). However, a match in magnitude can be considered an indication that the observed signal analysed in the regression is indeed the predicted signal, rather than, for instance, a response to an ignored driver that happens to closely resemble the predicted response to anthropogenic drivers. In this sense a match of magnitudes helps to build confidence. 
Within the regression formulation used here, the question is whether any of the regression coefficients for the anthropogenic response, $\beta_{\text {ant }}$ are inconsistent with 1 . This is a two-sided problem so we cannot use a similar approach as in Section 1.5.2. Instead, we consider the number,

$N_{\text {ant }}$, of $N_{\text {obs }} \cdot N_{\text {mod }}$ estimates of the $\beta_{\text {ant }}$ regression coefficient that are inconsistent with 1 at the $\alpha=0.1$ significance level, i.e. whether 1 lies outside of the $90 \%$ (statistical) confidence range. The choice of $\alpha=0.1$ simply follows the standard of most regression-based climate change studies (e.g. Bindoff et al 2013). The $p$-value $p_{a n t}$ of obtaining $N_{\text {ant }}$ failures in a binomial distribution centered on probability $\alpha=0.1$ is then used to calculate the multiplication factor of

$$
\gamma_{\text {ant }}=\frac{6}{10}+\frac{4}{10}\left(\frac{1}{2}\left(1-\cos \left(\pi \cdot p_{\text {ant }}\right)\right)\right)^{\zeta}
$$

where the exponent is defined such that the factor is halfway to its lowest value at $p_{\text {ant }}=\alpha$ (Figure 2 ). The one-sided nature reflects that our confidence is not diminished if fewer estimates of $\beta_{\text {ant }}$ are inconsistent with 1 than would be expected by chance (even though that could reflect an overestimation of uncertainty). The maximum penalty of $40 \%$ reflects a view that while a mismatch of magnitudes does indicate an inconsistency between predicted and observed responses, it does not necessarily interfere with detection (e.g. Gillett et al 2005).

The above test only concerns the response to anthropogenic drivers. While they are less directly connected to the conclusions of the analysis it would also help build confidence (or not reduce it), if the observed responses to natural drivers are also not inconsistent with the predicted response. The step described in Section 1.5.3 is repeated here but for the estimates of $\beta_{\text {nat }}$ and with a smaller maximum reduction, such that

$$
\gamma_{\text {nat }}=\frac{9}{10}+\frac{1}{10}\left(\frac{1}{2}\left(1-\cos \left(\pi \cdot p_{\text {nat }}\right)\right)\right)^{\zeta}
$$

The maximum $10 \%$ reduction reflects that this test is less relevant for conclusions regarding anthropogenic forcing. For instance, climate models predict short cool periods following large explosive volcanic eruptions, but the observed cooling is significantly smaller than predicted. While it seems current climate models have a problem in representing relevant feedbacks, the detection of a response to volcanic eruptions is nevertheless generally considered robust (Bindoff et al 2013).

\section{Consistency of autonomous variability}

As an extremely nonlinear system, the climate generates variability autonomously whether it is being influenced by external factors or not. If the assumptions behind the regression hold and all the important external drivers have been included in the $X_{\text {all }}(t)$ and $X_{\text {nat }}(t)$ pair, then the residual $R(t)$ from the regression should be indistinguishable from this autonomous variability. Simulations of climate models whose external drivers do not vary from year to year provide an estimate of what that variability should be on the multi-decadal time scale that is relevant here. A comparison of the $\mathrm{R}(\mathrm{t})$ arising from the regression and the variability in these unforced simulations is performed following Allen and Tett (1999). 
In many cases the residual is inconsistent with the unforced simulations at the $\alpha=0.1$ significance level. Following the same approach as in Section 1.5.4, the multiplication factor is defined as

$$
\gamma_{\text {resid }}=\frac{1}{2}+\frac{1}{2}\left(\frac{1}{2} \cdot\left(1-\cos \left(\pi \cdot p_{\text {resid }}\right)\right)\right)^{\zeta}
$$

If the residuals from all $\mathrm{N}_{\mathrm{obs}} \cdot \mathrm{N}_{\text {mod }}$ combinations fails the test, then the confidence metric is reduced up to three levels. As with inconsistencies in the regression coefficients, gross failure of the residual test is a major concern (especially as it is a weak test, Allen and Tett 1999), and could reflect missing drivers, amongst other possibilities.

\section{Major role}

Assessment of the attribution of observed climate change to anthropogenic emissions requires a description of the magnitude of that role relative to other factors (Hegerl et al 2010). For this algorithm we assess whether emissions have had a 'major role' in the behavior of the observed climate (Stone et al 2013). This is interpreted as asking whether the anthropogenic response accounts for at least one third of the temporal variance; other possible contributors to the variance would be the response to natural drivers, autonomous variability, or possible neglected drivers. This is calculated by integrating the variance of the adjusted anthropogenic response across the calculated probability distribution of the regression coefficient $\beta_{\text {ant }}$ :

$$
\gamma_{\text {major }}=\frac{\int_{\eta} \int_{t}\left(\beta_{\text {ant }}(\eta) \cdot X_{\text {ant }}(t)\right)^{2} \cdot d \eta \cdot d t}{\int_{t}\left(X_{\text {obs }}(t)\right)^{2} \cdot d t}
$$

where the $d \eta$ are the quantiles of the probability distribution of the $\beta_{\text {ant }}$ regression coefficient. Note that without this test of the relative role, this algorithm is assessing the detection of an anthropogenic response in observed climate change (Hegerl et al 2010; Stone et al 2013a).

\subsection{Mapping the quantitative metric to the qualitative levels}

The multiplication factors described in Sections 1.3 through 1.5 are multiplied together to produce the confidence metric:

$$
c=\gamma_{\text {sources }} \cdot \gamma_{\text {density }} \cdot \gamma_{\text {size }} \cdot \gamma_{\text {physics }} \cdot \gamma_{\text {sign }} \cdot \gamma_{\text {signal }} \cdot \gamma_{\text {ant }} \cdot \gamma_{\text {nat }} \cdot \gamma_{\text {resid }} \cdot \gamma_{\text {major }}
$$

This metric is then mapped to the confidence levels listed in Table 2 which include the five levels of Mastrandrea et al (2010) as well as a further level of no confidence $\left(c_{\operatorname{map}}=0\right)$ for cases where no evidence is available or the algorithm reveals a fundamental disagreement between expected and observed responses. The mapping is performed according to 


$$
c_{m}=\frac{5 \cdot \log (1+3 c)}{2 \cdot \log 4}
$$

The logarithmic nature of this mapping function reflects the multiplicative nature in which the tests modify the initial $\gamma_{\text {sources }}$ value. The constants serve two purposes. First, if $N_{\text {obs }}=N_{\text {mod }}=1$ and all $\mathrm{Y}$ multipliers are equal to 1 (i.e. all tests are passed perfectly, then $c_{m}=2.5$ and we have medium confidence of a major anthropogenic contribution. Second, adding one within the logarithm provides a lower bound of no confidence, but it distorts the logarithmic interpretation, which the factor of 3 within the logarithm alleviates for larger values

Table2: List of confidence levels described by Mastrandrea et al (2010) and the corresponding values of the quantitative confidence metric $c_{\text {map }}$ used in this paper. An additional level of no confidence is added for cases where no information is available.

\begin{tabular}{|l|l|}
\hline Confidence level & Numerical value \\
\hline No confidence & $\mathrm{c}_{\mathrm{m}}=\mathbf{0}$ \\
\hline Very low confidence & $0<\mathrm{c}_{\mathrm{m}}<1$ \\
\hline Low confidence & $1 \leq \mathrm{c}_{\mathrm{m}}<2$ \\
\hline Medium confidence & $\mathbf{2} \leq \mathrm{c}_{\mathrm{m}}<3$ \\
\hline High confidence & $\mathbf{3} \leq \mathrm{c}_{\mathrm{m}}<4$ \\
\hline Very high confidence & $\mathbf{4} \leq \mathrm{c}_{\mathrm{m}}$ \\
\hline
\end{tabular}

\section{Comparison against detailed assessments}

\subsection{Data}

In this section we compare attribution results from the algorithm described above against assessments in the IPCC AR5. The data required by the algorithm can be divided into observationally-based $\left(X_{\text {obs }}\right)$ and climate model-based $\left(X_{\text {all }}, X_{\text {nat }}\right.$, and $\left.X_{\text {noise }}\right)$. The observational data sets used are listed in Table 3, selected on the basis of having global (terrestrial or marine) coverage, covering the 1951-2010 period, and having a spatial resolution finer than $250^{2} \mathrm{~km}^{2}$. All of the terrestrial air temperature and precipitation data sets are based on in situ station monitoring, while the two marine data sets analyse both in situ measurements and, in more recent decades, remote sensing data. The Hurrell sea surface temperature data set adopts HadISST1 values through to October 1981, then NOAA Ol.v2 values (Reynolds and Smith 1994) thereafter. Because not all products report the monitoring density, only the data sets listed in Table 1 are used for that purpose.

The response signals $X_{\text {all }}(t)$ and $X_{n a t}(t)$, as well as the autonomous unforced variability $X_{\text {noise, }}$, can be estimated from simulations of dynamical climate models driven with only the respective external drivers. For this analysis, we take simulations from the CMIP5 database (Taylor et al 2012). The spatial resolution corresponds to the average box size on the grid used to output the data. This is the same grid or approximates the scale used in the model's dynamical calculations. Because of the nature of simulation numerics, models effectively only resolve features several times this scale; in this sense the spatial resolution is not directly comparable against the resolution listed for the observational data sets. For each of the models with multiple available simulations for estimating the response signals $X_{\text {all }}$ and $X_{\text {nat }}$, the sampling noise is reduced by averaging across the 3 to 10 simulations available in each case. The regression model is estimated for each combination of the 
$\mathrm{N}_{\text {mod }}=7$ climate models with available simulations and the $\mathrm{N}_{\mathrm{obs}}$ observational products, resulting in 21 regression models for 2 - $m$ air temperature over land, 14 for sea surface temperature, and 28 for precipitation. Data for estimating the autonomous unforced variability $X_{\text {noise }}$ is taken from these and additional climate models in order to allow a more precise estimate of the expected covariance of the residual $\mathrm{R}(\mathrm{t})$.

Table 3: List of observational data products used for analysis in this paper. On average, a $1^{\circ}$ longitude by $1^{\circ}$ latitude grid box covers $7900 \mathrm{~km}^{2}$ across the globe, being larger at the equator than at the poles

\begin{tabular}{l|l|c} 
Climate variable & Data products & Spatial resolution \\
\hline $2 \mathrm{~m}$ air temperature & CRU TS 3.22 (Harris et al. 2014) & $2000 \mathrm{~km}^{2}$ \\
& GISTEMP v6 (250 km land) (Hansen et al. 2010) & $63000 \mathrm{~km}^{2}$ \\
& UDel v3.01 (Matsuura \& Willmott 2012) & $2000 \mathrm{~km}^{2}$ \\
\hline Precipitation & CRU TS 3.22 (Harris et al. 2014) & $2000 \mathrm{~km}^{2}$ \\
& GPCC v6 (Schneider et al. 2014) & $7900 \mathrm{~km}^{2}$ \\
& NOAA PRECL (1x1 $1^{\circ}$ (Chen et al. 2002) & $7900 \mathrm{~km}^{2}$ \\
& UDel v3.01 (Matsuura \& Willmott 2012) & $2000 \mathrm{~km}^{2}$ \\
\hline Sea surface temperature & HadISST1 (Rayner et al. 2003) & $7900 \mathrm{~km}^{2}$ \\
& Hurrell (Hurrell et al. 2008) & $9200 \mathrm{~km}^{2}$
\end{tabular}

\subsection{IPCC AR5 WGI Chapter 10 Assessments}

A first comparison can be made against the assessments of regional warming from the climate change detection and attribution chapter of the IPCC AR5 (Bindoff et al 2013). The statements were intended to be robust for the regional domain and time period. We assume that all statements:

- refer exclusively to land territory (including the Arctic statement, due to the paucity of marine monitoring in the Arctic);

- are relevant to the 1951-2010 period (except for the Arctic for which the 1961-2010 period more closely matches the statement);

- apply to the IPCC regional definitions used in the IPCC AR5 (Hewitson et al 2014); and

- apply to annual mean values averaged evenly over the region.

Table 4 compares confidence assessments made with the algorithm developed in Section 1 against assessments for statements made in the IPCC AR5.

The algorithm agrees with the IPCC AR5 assessments for the attribution assessments for all of the populated regions except Africa, where the confidence metric falls just shy of the border between medium and high confidence. The station density step is the dominant difference between the African result and those for the other populated regions (not shown). The difference for the Antarctica conclusion similarly arises because the CRU TS 3.22 product used for estimating station density does not cover Antarctica. In contrast, the discrepancy for the Arctic conclusion arises mostly from the test of the residual variability after the regression. 
Table 4: Comparison of confidence in detection statements and in attribution statements for regional assessments in Table 10.1 in Bindoff et al (2013). The specific periods analysed (given in square brackets) are based on the discussion in Table 10.1 and Section 10.3.1.1.4

\begin{tabular}{|c|c|c|c|c|c|}
\hline & & \multicolumn{2}{|c|}{$\begin{array}{ll}\text { Confidence } & \text { in } \\
\text { detection } & \end{array}$} & \multicolumn{2}{|c|}{$\begin{array}{l}\text { Confidence in attribution } \\
\text { of a major role }\end{array}$} \\
\hline \multicolumn{2}{|c|}{ WGI AR5 (Bindoff et al. 2013) results } & WGI AR5 & Algorithm & WGI AR5 & Algorithm \\
\hline \multirow{6}{*}{$\begin{array}{l}\text { Result 28: } \\
\text { "Anthropogenic forcing } \\
\text { has made a substantial } \\
\text { contribution to } \\
\text { warming to each of the } \\
\text { inhabited continents" }\end{array}$} & Africa & & & high & medium \\
\hline & Europe & & & high & high \\
\hline & Asia & & & high & high \\
\hline & Australasia & & & high & high \\
\hline & North America & & & high & high \\
\hline & $\begin{array}{l}\text { South and Central } \\
\text { America }\end{array}$ & & & high & high \\
\hline \multicolumn{2}{|c|}{$\begin{array}{l}\text { Result 29: "Anthropogenic contribution to very } \\
\text { substantial Arctic warming over the past } 50 \text { years } \\
\text { [1961-2010]" }\end{array}$} & high & medium & & \\
\hline \multicolumn{2}{|c|}{$\begin{array}{l}\text { Result 30: "Human contribution to observed } \\
\text { warming averaged over available stations over } \\
\text { Antarctica [1951-2010]" }\end{array}$} & low & no & & \\
\hline
\end{tabular}

\subsection{IPCC AR5 WGII Chapter 22 Assessments}

A further comparison can be made against the subcontinental detection and attribution assessments made in the African chapter of the IPCC AR5 (Niang et al 2014). Niang et al (2014) provide assessments for both precipitation and temperature, both of detection and of attribution of a major role for five regions based on the Regional Economic Communities. While no time period is specified, the assessments are heavily influenced by the time series plots of observed and simulated change shown in the chapter (which happen to share a large number of data sources with this manuscript); given these plots and that monitoring density improves markedly in the 1960s, we take the relevant period to be 1961-2010. Additionally, while the IPCC AR5 precipitation assessments are for land areas only, the temperature assessments apply to the combined terrestrial and Exclusive Economic Zone (EEZ) territories. Because of the lack of high spatial resolution observational products of combined terrestrial and marine near-surface air temperature, we must treat the terrestrial and marine areas separately. Thus we assume that the assessments apply equally to these two components of the overall region. The comparison is listed in Table 5.

Unlike for the comparison in Section 2.2, there are more numerous discrepancies for the African regions. Precipitation assessments are all the same or less confident than in the IPCC AR5. For majorrole attribution, the inconsistency for four regions could arise simply from the lack of a no confidence level in the IPCC AR5. The only two-level discrepancy is for rainfall over ECOWAS (the Economic Community of West African States). In Niang et al (2014) the ECOWAS assessment was based on a number of detailed studies which considered the underlying data sources and processes in more detail than in our algorithm, for instance evaluating and selecting climate models based on their ability to adequately represent the West African monsoon. However, many of these studies specifically examined the drying and partial recovery of rainfall over the Sahel, which only partly overlaps with the ECOWAS region (note that the spatial averaging of precipitation performed here is based on fractional anomalies, so a $30 \%$ reduction in some part of the Sahel would be considered equivalent to a $30 \%$ reduction in a corresponding area on the much wetter coast). This case may therefore be illustrating both the relative strength of detailed targeted analysis in comparison to this paper's 
algorithm, and the relative strength of the algorithm in being easily tailored to the specific requirement of an assessment (in this case the ECOWAS territorial area).

Table 5: Comparison of confidence in detection and attribution statements for the African regions listed in Figure 22-3 of (Niang et al 2014). The regions are based on the Regional Economic Communities: the combination of the East African Community, Intergovernmental Authority on Development, and Egypt (EAC/IGAD/Egypt); the Economic Community of Central African States (ECCAS); the Economic Community of West African States (ECOWAS); the Southern African Development Community (SADC); and the Arab Maghreb Union (UMA). Niang et al (2014)'s assessments of warming applied to the combination of land and Exclusive Economic Zone (EEZ) areas, whereas these two components are assessed separately here

\begin{tabular}{|c|c|c|c|c|}
\hline \multirow[b]{2}{*}{$\begin{array}{l}\text { IPCC WGII Africa Chapter result (Niang et al. } \\
\text { 2014) }\end{array}$} & \multicolumn{2}{|c|}{ Confidence in detection } & \multicolumn{2}{|c|}{$\begin{array}{l}\text { Confidence in attribution of } \\
\text { major role }\end{array}$} \\
\hline & IPCC WGII & Algorithm & IPCC WGII & Algorithm \\
\hline $\begin{array}{l}\text { EAC/IGAD/Egypt land annual mean precipitation } \\
\text { changes }\end{array}$ & low & low & very low & no \\
\hline $\begin{array}{l}\text { EAC/IGAD/Egypt land and EEZ annual mean } \\
\text { warming }\end{array}$ & medium & $\begin{array}{l}\text { high (land) } \\
\text { medium (EEZ) }\end{array}$ & medium & $\begin{array}{l}\text { high (land) } \\
\text { medium (EEZ) }\end{array}$ \\
\hline ECCAS land annual mean precipitation changes & very low & very low & very low & no \\
\hline ECCAS land and EEZ annual mean warming & low & $\begin{array}{l}\text { very low (land) } \\
\text { medium (EEZ) }\end{array}$ & low & $\begin{array}{l}\text { very low (land) } \\
\text { medium (EEZ) }\end{array}$ \\
\hline ECOWAS land annual mean precipitation changes & medium & low & low & no \\
\hline ECOWAS land and EEZ annual mean warming & medium & $\begin{array}{l}\text { high (land) } \\
\text { high (EEZ) }\end{array}$ & medium & $\begin{array}{l}\text { high (land) } \\
\text { high (EEZ) }\end{array}$ \\
\hline SADC land annual mean precipitation changes & low & very low & very low & no \\
\hline SADC land and EEZ annual mean warming & high & $\begin{array}{l}\text { medium (land) } \\
\text { high (EEZ) }\end{array}$ & high & $\begin{array}{l}\text { medium (land) } \\
\text { high (EEZ) }\end{array}$ \\
\hline UMA land annual mean precipitation changes & very low & very low & very low & no \\
\hline UMA land and EEZ annual mean warming & high & $\begin{array}{l}\text { medium (land) } \\
\text { medium (EEZ) }\end{array}$ & medium & $\begin{array}{l}\text { medium (land) } \\
\text { medium (EEZ) }\end{array}$ \\
\hline
\end{tabular}

Marine temperature detection and major-role attribution assessments both tend to be assigned higher confidence by the algorithm, whereas discrepancies tend to balance for terrestrial temperature. In part this is a reflection of the separation of the joint terrestrial-marine assessments: some of the terrestrial assessments suffer from poor station coverage, so when the marine areas are separated they are freed from this penalty. ECCAS (the Economic Community of Central African States) suffers especially from poor monitoring coverage over land, but not over its EEZ.

\subsection{Application to nation-scale regions}

In this section we deploy the algorithm on precipitation and temperature changes over a large number of regions. We adopt the regions developed for an operational system linking change in the chance of extreme weather to anthropogenic emissions (http://www.csag.uct.ac.za/ daithi/forecast; Angélil et al 2014a,b). These regions are based on political/economic groupings and are all approximately 2 
million $\mathrm{km}^{2}$ in size. Because of limitations in the modelling technique used by that system, all regions are terrestrial and exclude countries dominated by archipelagos (e.g. Indonesia) as well as Antarctica.

The algorithm is run on annual total precipitation and annual mean $2 \mathrm{~m}$ air temperature over these regions. (As in Section 2.3, the spatial averaging of precipitation is on the fractional anomaly, so arid and wet areas contribute equally to a region's variability.) The data sources are the same as listed in Tables 1 and 3, and the test comparing observed against required trends $\left(\gamma_{\text {sign }}\right)$ is omitted.

The results for detection of an anthropogenic influence and for attribution of a major anthropogenic role (differing through the exclusion/inclusion of $Y_{\text {major }}$ from the 'major role' test) are shown in Figure 3.

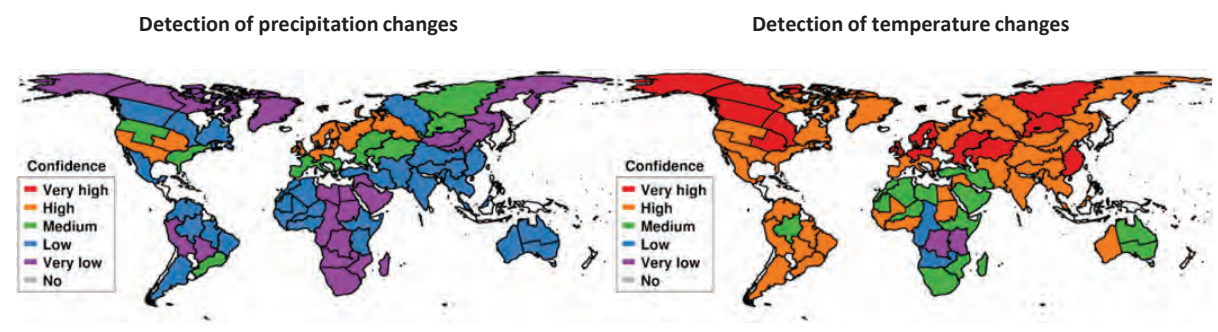

Attribution of major role in precipitation changes

Attribution of major role in temperature changes

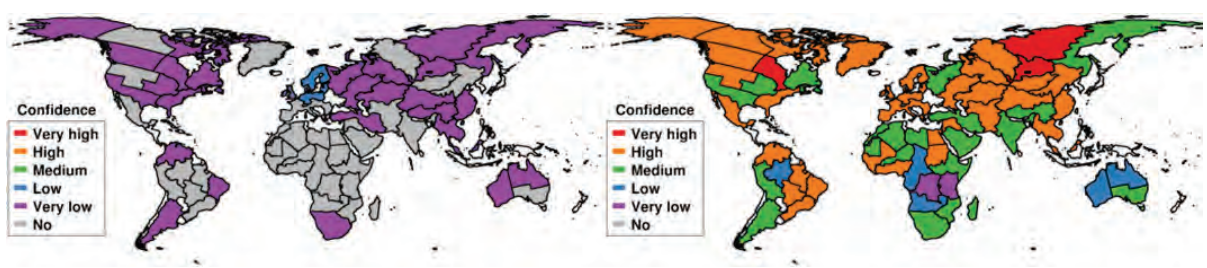

Figure 3: Assessments of the detection of an influence (top row) and attribution of a major role (bottom role) for anthropogenic emissions in observed climate variations during the 1961-2010 period over various political/economic regions of the world. All regions are terrestrial and approximately 2 million $\mathbf{k m}^{2}$. All assessments are for annual averages.

An anthropogenic influence on precipitation variations is only detected with a reasonable confidence in some northern mid and high latitude regions, and with one exception (consistent with the recent assessment report for part of that region (Bhend 2015) there is at most very low confidence that that role is substantial. The reason for this is apparent in Figure 4, which shows how the estimate of confidence is affected by the various steps of the algorithm. For a large number of the regions, inadequate station density in the monitoring networks is a major restriction on confidence in detection. Note that because of the smaller decorrelation scale the network density must be considerably higher for precipitation than for temperature (Table 1). Unlike for temperature, all of the steps in the algorithm contribute to a notable decrease in the confidence metric for detection of precipitation changes over almost all regions. The magnitude of any potentially detected signal is always very small in relation to the autonomous year-to-year variability of the climate system, leading to consistently large decreases in confidence at the 'major role' step distinguishing detection of an anthropogenic influence from attribution of a major role. 
Not surprisingly, confidence is much higher for temperature changes, with at least high confidence in detection of an anthropogenic influence over most regions outside of Africa and slightly less confidence of attribution of a major role (Figure 3). The spatial pattern of confidence in detection (and attribution) differs from the expected signal-to-noise ratio (Mahlstein et al 2011; Bindoff et al 2013). The reason for this is apparent from Figure 3. While all four regions with low or very low confidence in detection are located in an area of the tropics with an expected high signal-to-noise ratio, they are also regions with poor monitoring station coverage; monitoring coverage tends to be a major factor for regions with medium confidence in detection as well. This illustrates that the current rule-of-thumb of stronger detectability of warming in the tropics (Bindoff et al 2013) ignores the full set of sources of uncertainty, and in particular the role of adequate long-term monitoring.

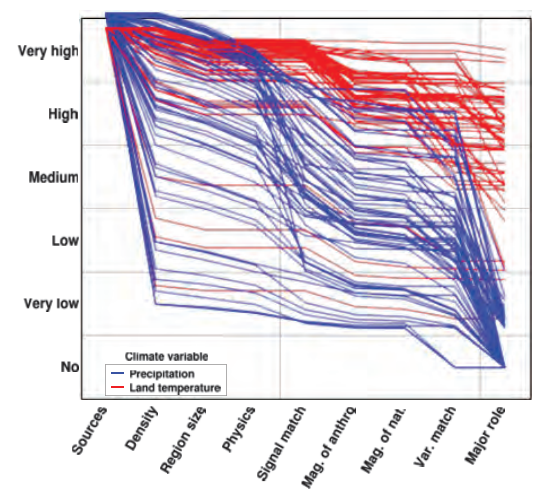

Figure 4: Calculation of the confidence metric for the assessments shown in Figure 3 of the detection and attribution of observed climate variations during the 1961-2010 period for various 2 million km2 political/economic regions of the world.

\section{Discussion}

This manuscript has both developed a framework for assessing the detection and attribution of climate change on a large scale and developed a specific implementation. The framework is an explicit quantification of the framework developed and applied in recent IPCC Assessment Reports (Seneviratne et al 2012; Bindoff et al 2013; Cramer et al 2014), based on the confidence level formulation of Mastrandrea et al (2010). This considers not only the result of a single comparison of expected responses to climate change with observed trends, but also examines the agreement between comparisons using different data sources, as well as the underlying appropriateness and accuracy of those data sources. In terms of implementation in the IPCC reports, it has generally been assumed that the components of confidence are separable. What is new in the algorithm developed in this manuscript is the concept that the components may also be quantifiable and multiplicative. The degree to which both these assumptions are justifiable is open to discussion, but for this algorithm the assumptions only need to hold approximately, because the algorithm is not intended for use as an expert 'final word' but rather a more general tool.

In terms of the specific implementation developed in this manuscript, it is centred around the popular multiple linear regression approach underlying much climate change detection and attribution research (Bindoff et al 2013), but other options exist. Indeed, exploration of multiple methods should really be considered in establishing confidence. Similarly the division of the 
components of confidence could probably be chosen differently and may be affected by the exact nature of the detection and attribution study. For instance, the test of whether the observed trend matches a reported trend (Section 1.4) was not applicable in the tests and examples presented in the previous two sections.

There are three specific areas of the implementation that require consideration. First, the algorithm operates within a realm of well-behaving inputs. For instance, while it is plausible, as stipulated in Section 1.3, that the maximum possible confidence should be the same when $\left[\mathrm{N}_{\mathrm{obs}}=3, \mathrm{~N}_{\bmod }=6\right]$ as when $\left[\mathrm{N}_{\mathrm{obs}}=6, \mathrm{~N}_{\text {mod }}=3\right]$, it is less obvious that the situation $\left[\mathrm{N}_{\mathrm{obs}}=36, \mathrm{~N}_{\bmod }=1\right]$ or $\left[\mathrm{N}_{\mathrm{obs}}=1, \mathrm{~N}_{\bmod }=\right.$ 36] should also have the same maximum confidence. Modifications may be necessary for such extreme situations.

Second, the quality of the development of the various steps in the algorithm varies considerably. For instance, confidence in detection must depend directly on the station density as well as the distribution of those stations, and there must be no confidence when there are no observations at all (Hegerl et al 2010). While the method used here makes simple assumptions about the spatial correlation of climatic variability, these same assumptions are well tested and have also been used in the development of respected observational products. However, the way in which a statistical failure of the "variability match" step in the regression analysis should be translated into a quantitative modification of the confidence metric is less obvious. While selection of the maximum possible reduction in confidence by this step has been informed by experience with regression analysis, the value is still a subjective choice based on the authors' experience and intuition. Fortunately, the steps that seem to have the greatest bearing on the final confidence (monitoring density, signal match, and major role) are also the steps with the highest quality translation from analysis to confidence metric.

Finally, the output of this algorithm is qualitative in nature and the relation between qualitative terms like 'major role' and quantitative metrics remains at least partly subjective. Fortunately, results here do not appear to have been that sensitive to choice of 'major role' threshold. However, the mapping from the quantitative confidence metric $c$ through $c_{\text {map }}$ into the qualitative levels can lead to systematic shifts of a full confidence level. In that sense the comparisons performed in Section 2 against existing expert assessments served an important role in check on the calibration of the algorithm.

Ultimately, this algorithm is no substitute for detailed expert detection and attribution analysis. For instance, the 'physical representation' step (Section 1.3.4 is the only step in the algorithm responsible for evaluating whether the climate models are capable of representing the processes required in order to adequately represent the regional and seasonal climate of interest. Currently that step consists of a simple binary function depending only on the climate variable. Future development of the algorithm could add some evaluation of, for instance, the spatial pattern of the mean annual climatology and the annual cycle, but it is hard to envisage a systematic approach that could ever be as nuanced and detailed as an expert evaluation whilst remaining generalizable.

Despite these disadvantages relative to detailed expert assessments, this algorithm has some important strengths that mean it can serve as a complementary tool. Most particularly, it can be deployed simply and straightforwardly on an industrial scale, as illustrated in Section 2.3. Performing those 116 assessments in 116 separate detailed papers would require unobtainable resources; in contrast, the main performance bottleneck in conducting the calculations presented in 
Section 2.3 was simply the extraction of the regional and seasonal data from the various data products. While there were some discrepancies between the algorithm's results and those of existing expert assessments, these may not have always pointed to a fault of the algorithm: some of the expert assessments were based on fewer data sources, for instance. Given these points, this algorithm, or approaches similar to it, could provide an important tool toward the inclusion of detection and attribution analysis within the provision of climate services.

\section{References}

Allen MR, Stott PA (2003) Estimating signal amplitudes in optical fingerprinting, part I:theory. Clim Dyn 21:477-491.

Allen MR, Tett SFB (1999) Checking for model consistency in optimal fingerprinting. ClimDyn 15:419434.

Angélil O, Stone DA, Pall P (2014a) Attributing the probability of South African weather extremes to anthropogenic greenhouse gas emissions: spatial characteristics. Geophy Res Lett 41:3238-3243.

Angélil O, Stone DA, Tadross M, Tummon F, Wehner M, Knutti R (2014b) Attribution of extreme weather to anthropogenic greenhouse gas emissions: sensitivity to spatial and temporal scales. Geophys Res Lett 41:2150-2155.

Bhend J (2015) Regional evidence of global warming. In: BACC II Author Team (ed) Second assessment of climate change for the Baltic Sea basin, Regional Climate Studies, Springer-Verlag, pp 427-439.

Bindoff NL, Stott PA, AchutaRao KM, et al (2013) Detection and attribution of climate change: from global to regional. In: Stocker TF, Qin D, Plattner GK, et al (eds) Climate Change 2013: The Physical Science Basis. Contribution of Working Group I to the Fifth Assessment Report of the Intergovernmental Panel on Climate Change, Cambridge University Press, pp 867-952.

Chen M, Xie P, Janowiak JE, Arkin PA (2002) Global land precipitation: A 50-yr monthly analysis based on gauge observations. J Hydrometeor 3:249-266.

Cramer W, Yohe GW, Auffhammer M, et al (2014) Detection and attribution of observed impacts. In: Field CB, Barros VR, Dokken DJ et al (eds) Climate Change 2014: Impacts, Adaptation, and Vulnerability. Part A: Global and Sectoral Aspects. Contribution of Working Group II to the Fifth Assessment Report of the Intergovernmental Panel on Climate Change, Cambridge University Press, pp 979-1037.

Gillett NP, Allan RJ, Ansell TJ (2005) Detection of external influence on sea level pressure with a multi-model ensemble. Geophys Res Lett 32:L19714.

Hansen J, Ruedy R, Sato M, Lo K (2010) Global surface temperature change. Rev Geophys 48:RG4004.

Harris I, Jones PD, Osborn TJ, Lister DH (2014) Updated high-resolution grids of monthly climatic observations - the CRU TS3.10 dataset. Int J Climatol 34:623-642.

Hegerl G, Zwiers F (2011) Use of models in detection and attribution of climate change. WIREs Climate Change 2:570-591. 
Hegerl GC, Hoegh-Guldberg O, Casassa G, et al (2010) Good practice guidance paper on detection and attribution related to anthropogenic climate change. In: Stocker TF, Field CB, Qin D, et al (eds) Meeting Report of the Intergovernmental Panel on Climate Change Expert Meeting on Detection and Attribution of Anthropogenic Climate Change, IPCC Working Group I Technical Support Unit, University of Bern, Bern, Switzerland.

Hewitson B, Janetos AC, Carter TR, et al (2014) Regional context. In: Barros VR, Field CB, Dokken DJ et al (eds) Climate Change 2014: Impacts, Adaptation, and Vulnerability. Part B: Regional Aspects. Contribution of Working Group II to the Fifth Assessment Report of the Intergovernmental Panel on Climate Change, Cambridge University Press, pp 1133-1197.

Hurrell JW, Hack JJ, Shea D, Caron JM, Rosinski J (2008) A new sea surface temperature and sea ice boundary dataset for the Community Atmosphere Model. J Climate 21:5145-5153.

Jones GS, Stott PA, Christidis N (2013) Attribution of observed historical near-surface temperature variations to anthropogenic and natural causes using CMIP5 simulations. J Geophys Res 118:40014024.

Jones PD, Osborn TJ, Briffa KR (1997) Sampling errors in large-scale temperature averages. J Climate 10:2548-2568.

Jun M, Knutti R, Nychka DW (2008) Spatial analysis to quantify numerical model bias and dependence: how many climate models are there. Journal of the American Statistical Association 103:934-947.

Kennedy JJ, Rayner NA, Smith RO, Saunby M, Parker DE (2011a) Reassessing biases and other uncertainties in sea-surface temperature observations since 1850 part 1: measurement and sampling errors. J Geophys Res 116:D14103.

Kennedy JJ, Rayner NA, Smith RO, Saunby M, Parker DE (2011b) Reassessing biases and other uncertainties in sea-surface temperature observations since 1850 part 2: biases and homogenisation. J Geophys Res 116:D14104.

Mahlstein I, Knutti R, Solomon S, Portmann RW (2011) Early onset of significant local warming in low latitude countries. Environ Res Lett 6:034009.

Mastrandrea MD, Field CB, Stocker TF, et al (2010) Guidance note for Lead Authors of the IPCC Fifth Assessment Report on consistent treatment of uncertainties. Intergovernmental Panel on Climate Change (IPCC), available at http://www.ipcc.ch.

Matsuura K, Willmott CJ (2012) Terrestrial air temperature and precipitation: 1900-2010 gridded monthly time series (v3.01). Technical repository, University of Delaware, available at http://climate.geog.udel.edu/ climate/html pages/Global2011/index.html.

New M, Hulme M, Jones P (2000) Representing twentieth-century space-time climate variability. Part II: Development of 1901-96 monthly grids of terrestrial surface climate. J Climate 13:2217-2238.

Niang I, Ruppel OC, Abdrabo MA, et al(eds) Africa. In Barros VR, Field CB, Dokken DJ et al (eds) Climate Change 2014: Impacts, Adaptation, and Vulnerability. Part B: Regional Aspects. Contribution of Working Group II to the Fifth Assessment Report of the Intergovernmental Panel on Climate Change, Cambridge University Press, pp 1199-1265.

Pennell C, Reichler T (2010) On the effective number of climate models. J Climate 24:2358-2367. 
Rayner NA, Parker DE, Horton EB, Folland CK, Alexander LV, Rowell DP, Kent EC, Kaplan A (2003) Global analyses of sea surface temperature, sea ice, and night marine air temperature since the late nineteenth century. J Geophys Res 108:4407.

Reynolds RW, Smith TM (1994) Improved global sea surface temperature analysis using optimum interpolation. J Climate 7:929-948.

Schneider U, Becker A, Finger P, Meyer-Christoffer A, Ziese M, Rudolf B (2014) GPCC's new land surface precipitation climatology based on quality-controlled in situ data and its role in quantifying the global water cycle. Theoretical and Applied Climatology 115:15-40.

Seneviratne SI, Nicholls N, Easterling D, et al (2012) Changes in climate extremes and their impacts on the natural physical environment. In: Field CB, Barros V, Stocker TF, et al (eds) Managing the Risks of Extreme Events and Disasters to Advance Climate Change Adaptation, Cambridge University Press, Cambridge, U.K., pp 109-230.

Shiogama H, Stone DA, Nagashima T, Nozawa T, Emori S (2012) On the linear additivity of climate forcing-response relationships at global and continental scales. International Journal of Climatology $33: 2542-2550$.

Stone D, Auffhammer M, Carey M, et al (2013) The challenge to detect and attribute effects of climate change on human and natural systems. Clim Change 121:381-395.

Taylor KE, Stouffer RJ, Meehl GA (2012) An overview of CMIP5 and the experiment design. Bull Amer Met Soc 93:485-498. 


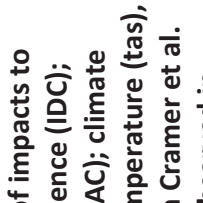

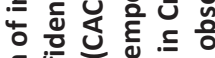

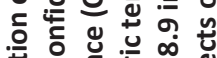
든 은 은 은 究 它

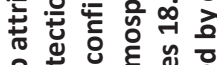

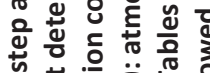

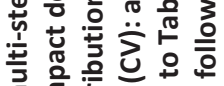
छ.

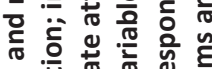
ธ。ํㅡㄹ 을

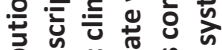

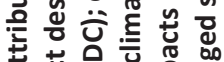

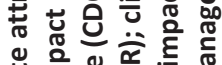

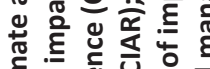

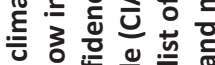

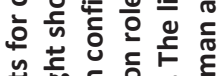

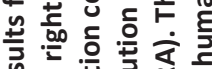

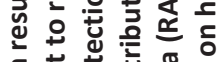

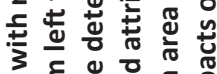

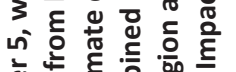

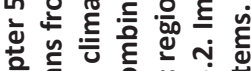
응 穴 ป

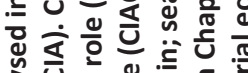

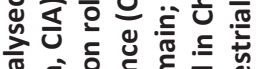

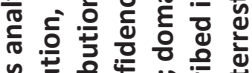

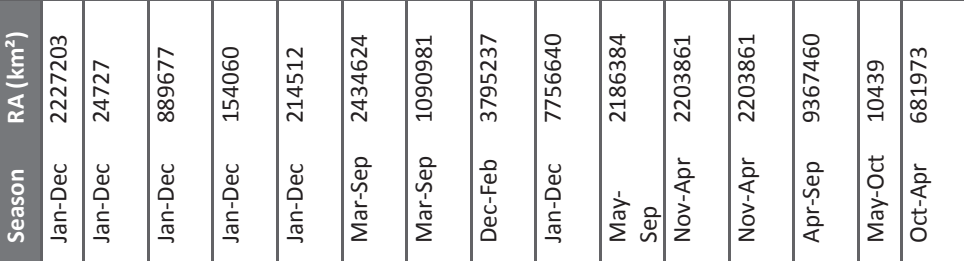

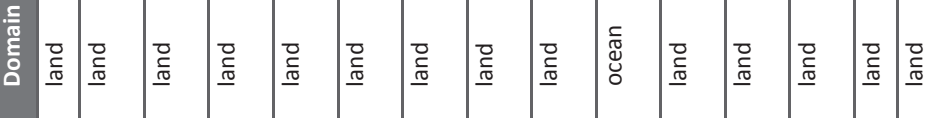
ひ立 委

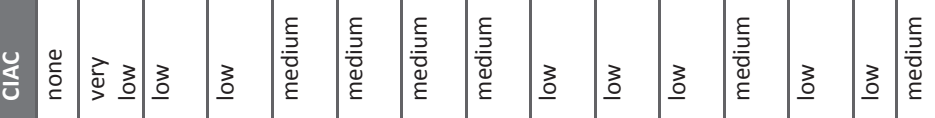
孚

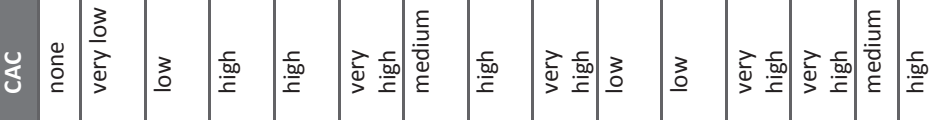

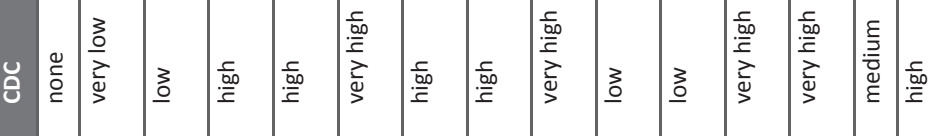

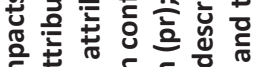

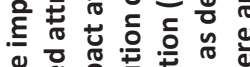

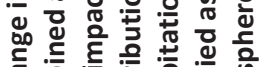

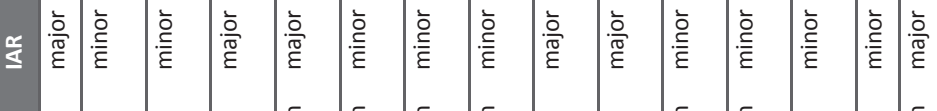

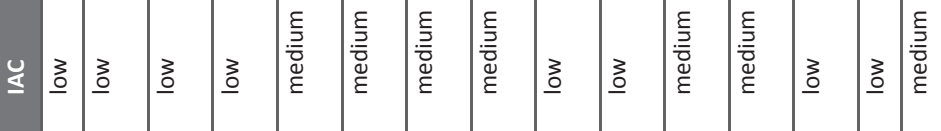

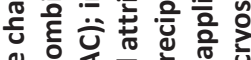

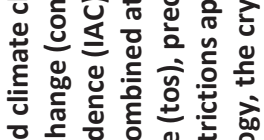

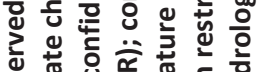

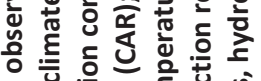

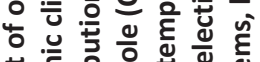

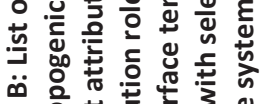
希总芯 


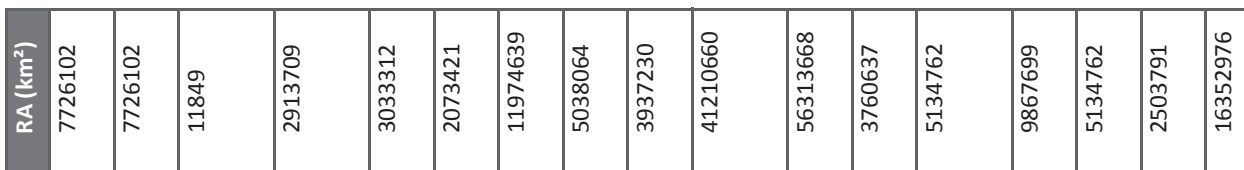

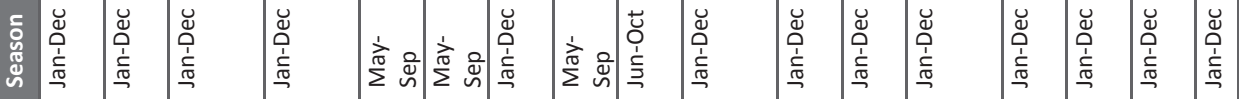

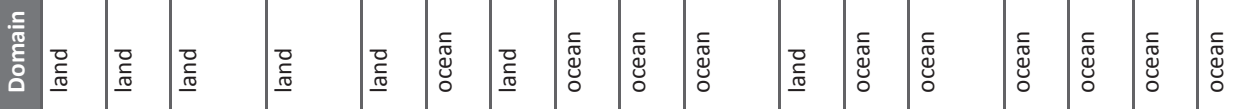

乙弯

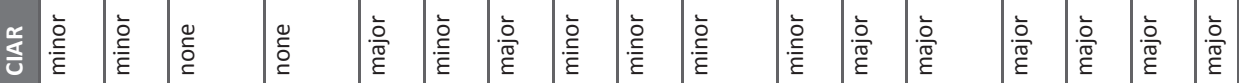

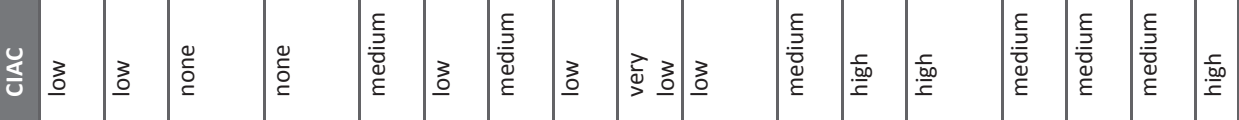

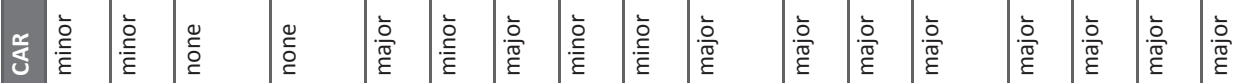

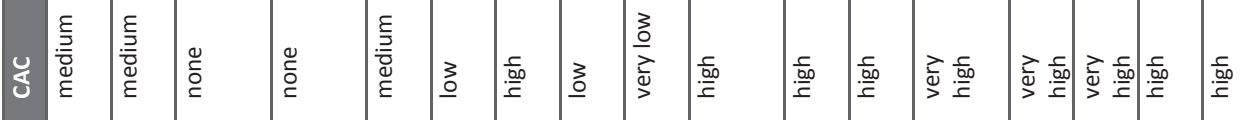

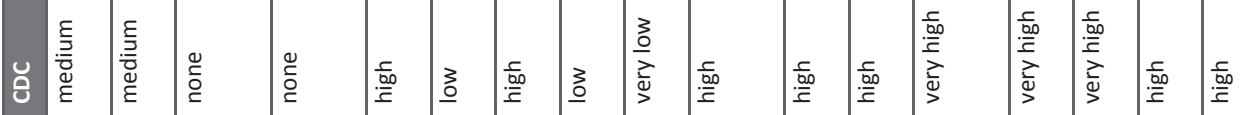

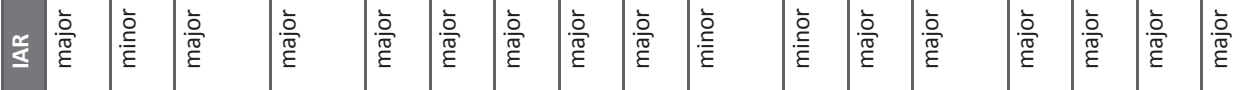

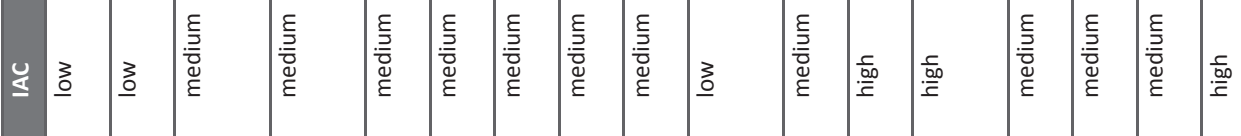

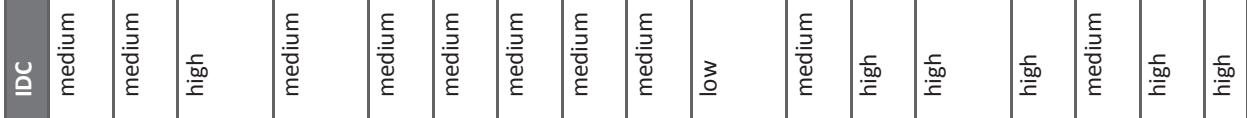

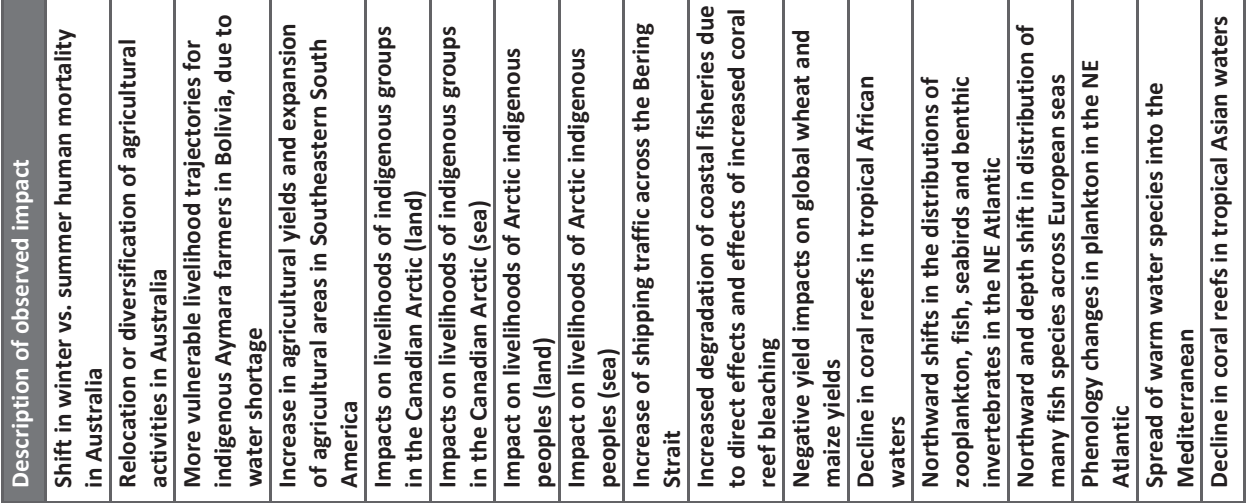




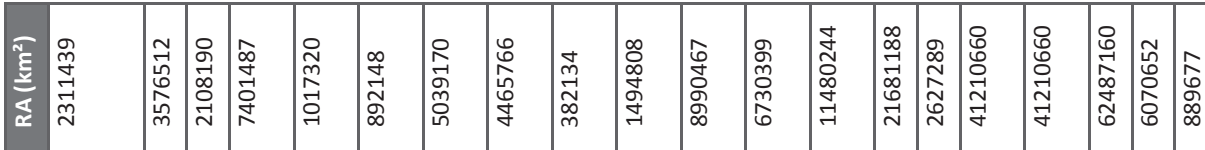

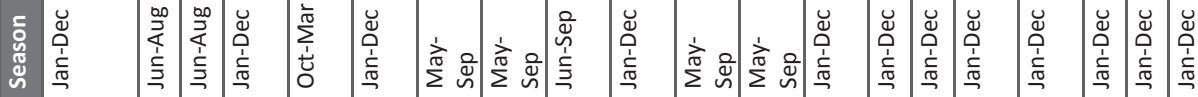

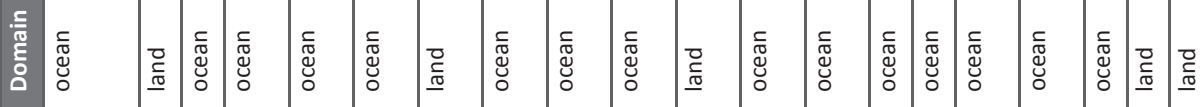

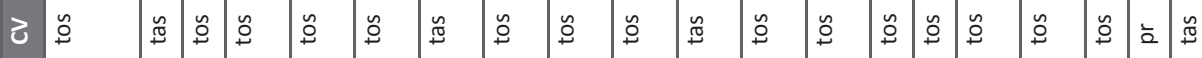

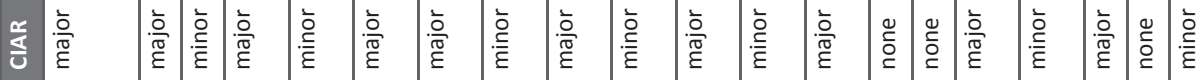

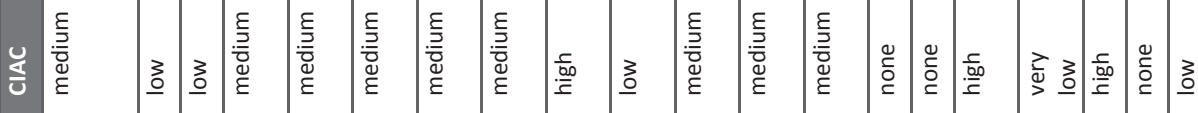

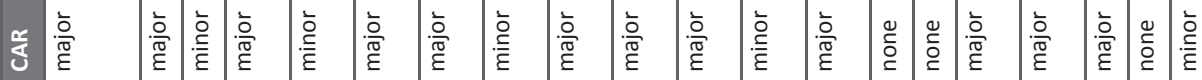

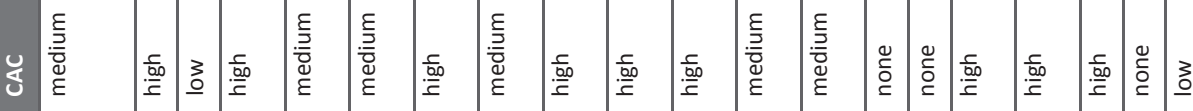

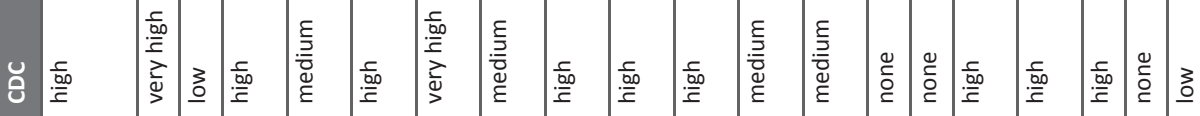

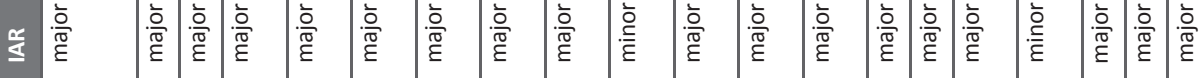

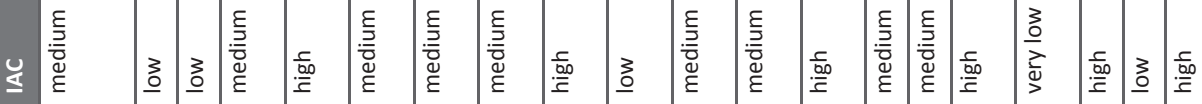

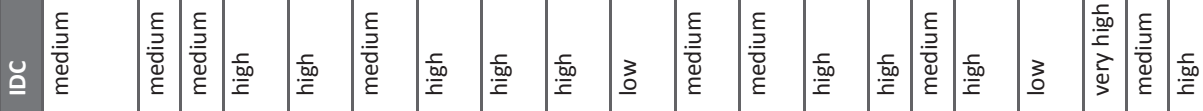

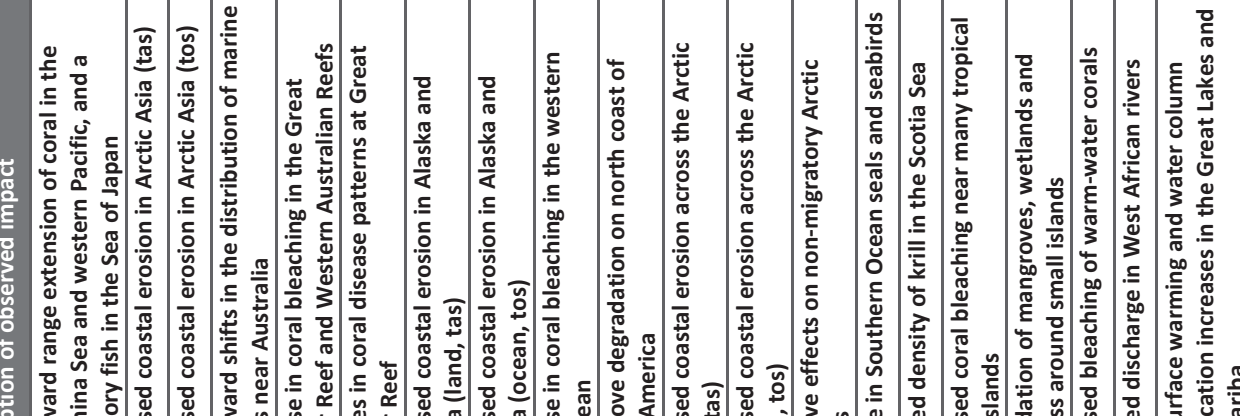

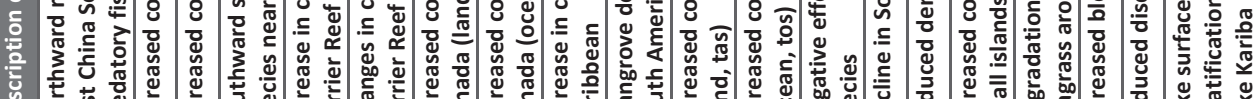

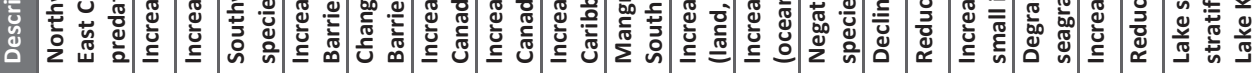




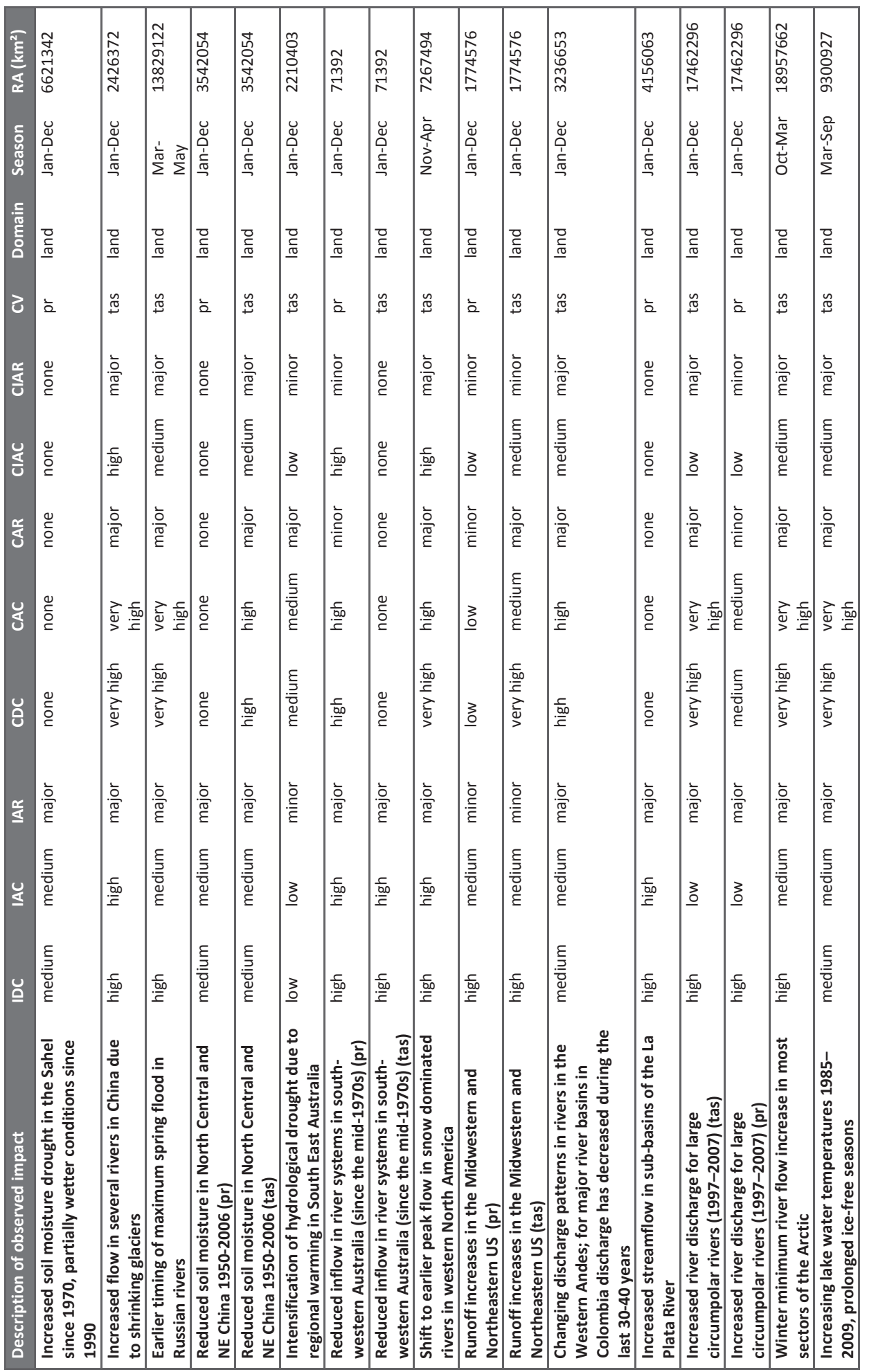




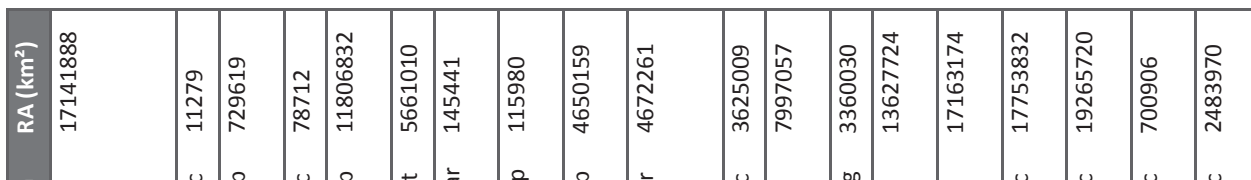

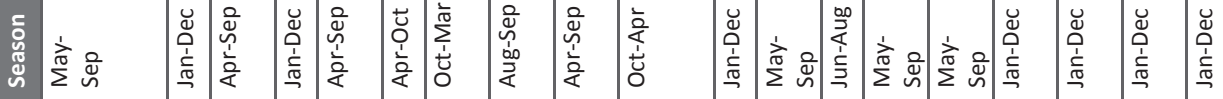

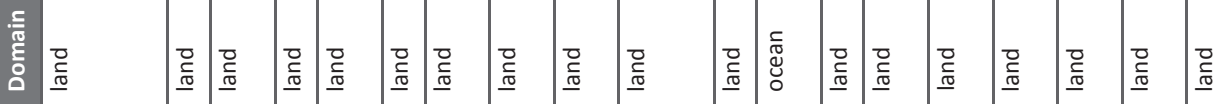
乙弯立

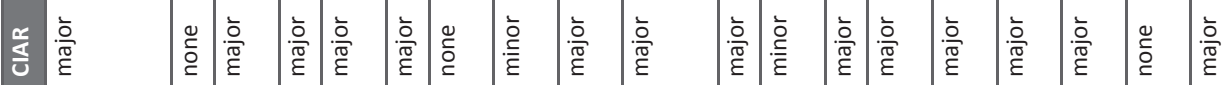

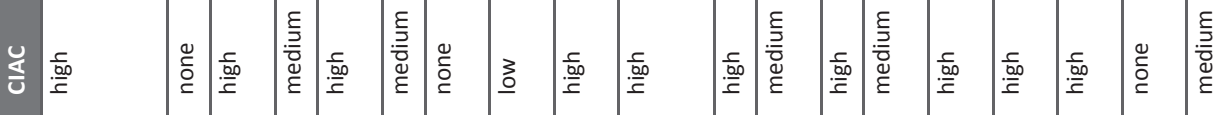

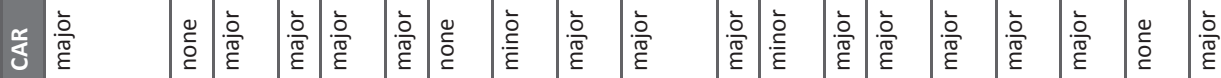

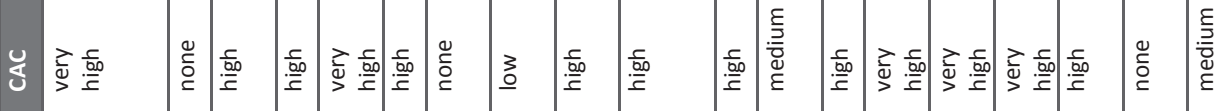

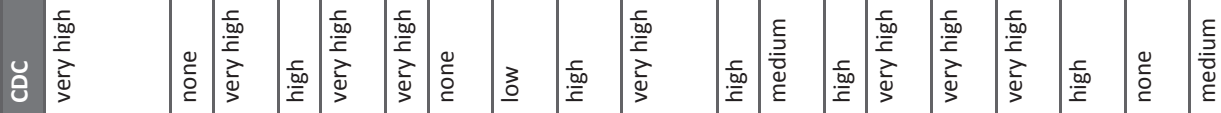

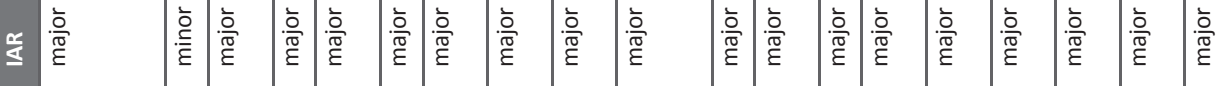

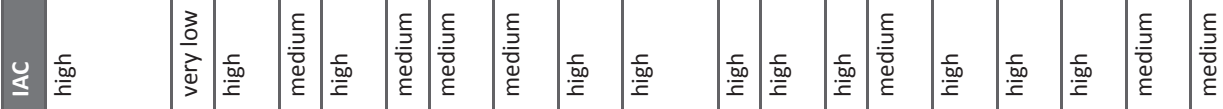

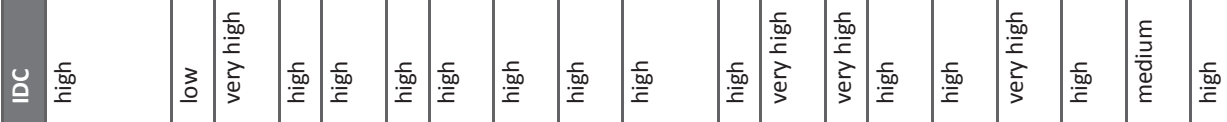

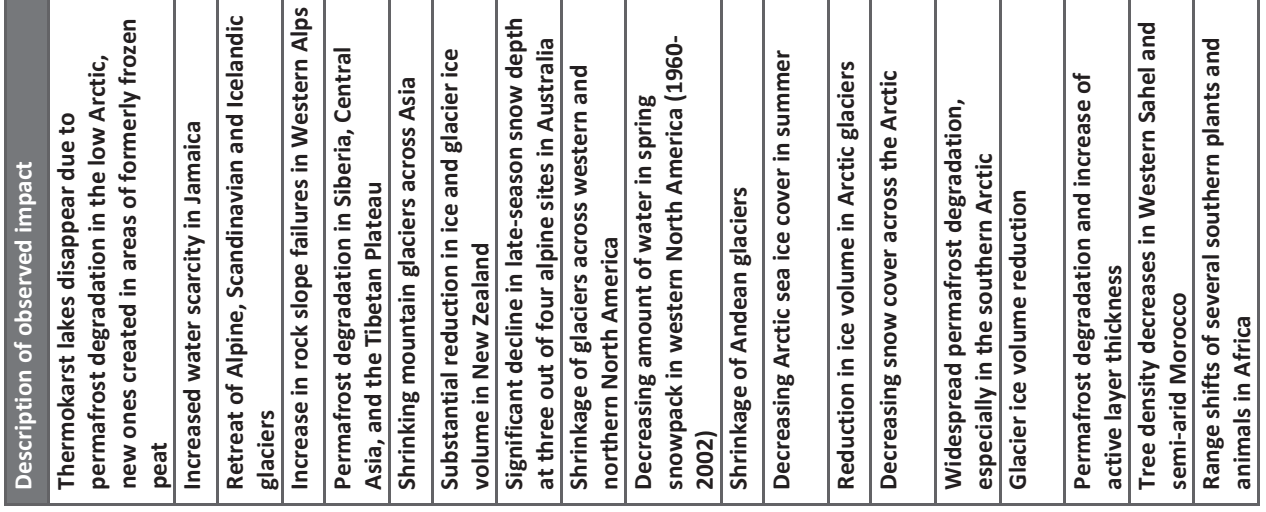




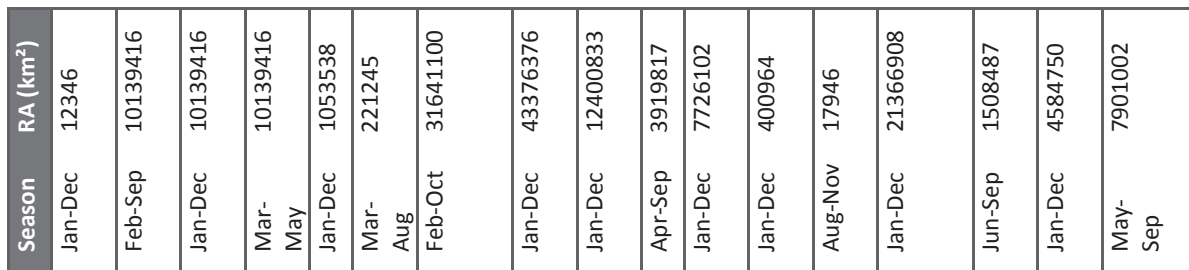

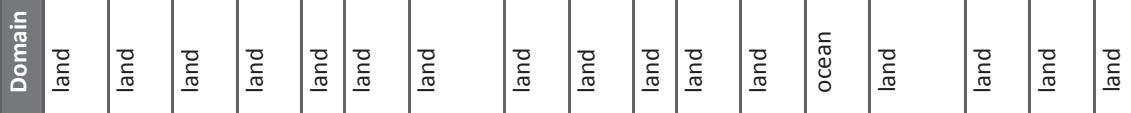

己焉

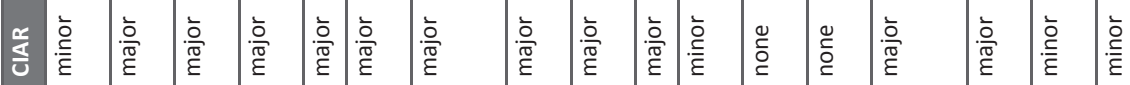

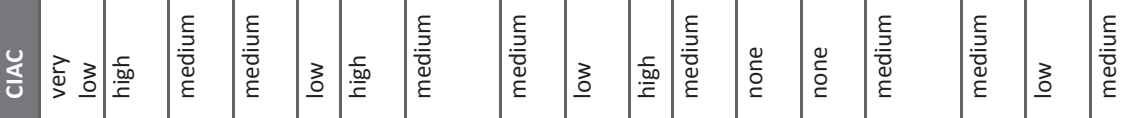

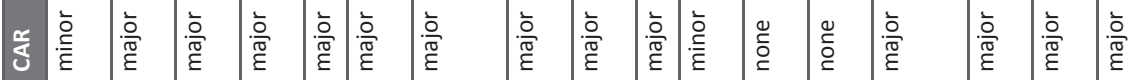

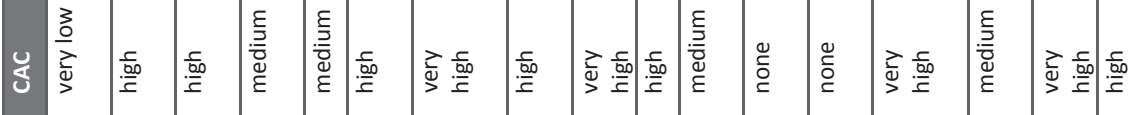

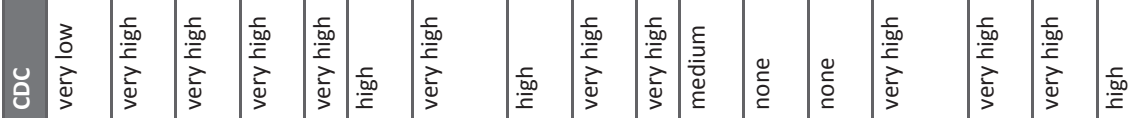

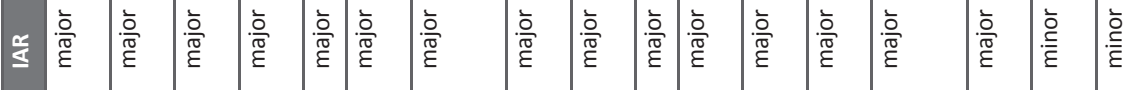

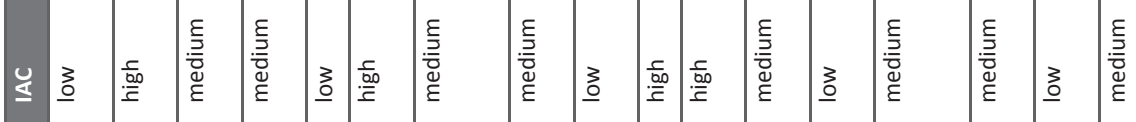

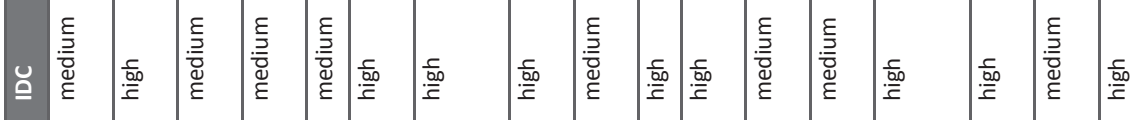

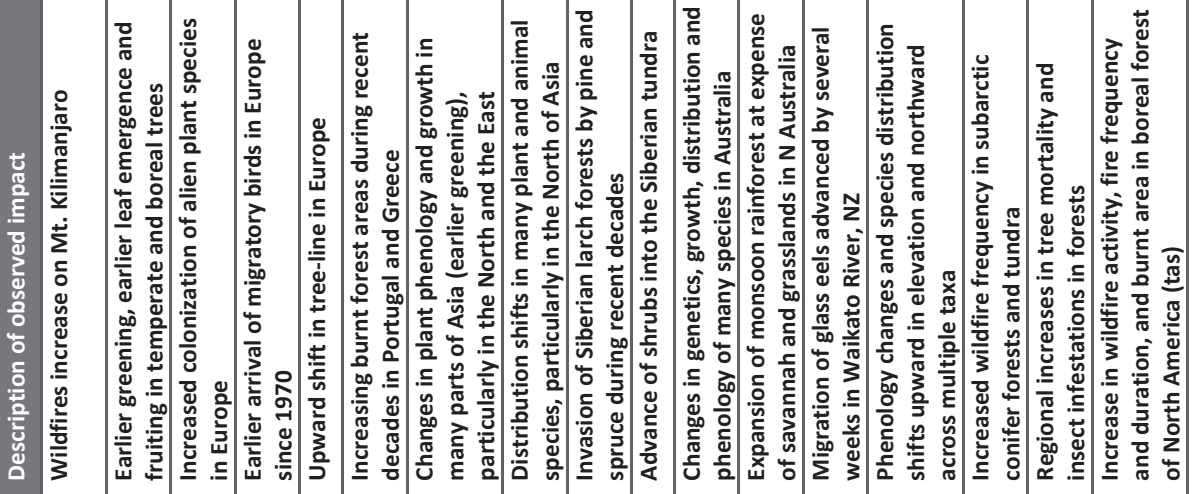




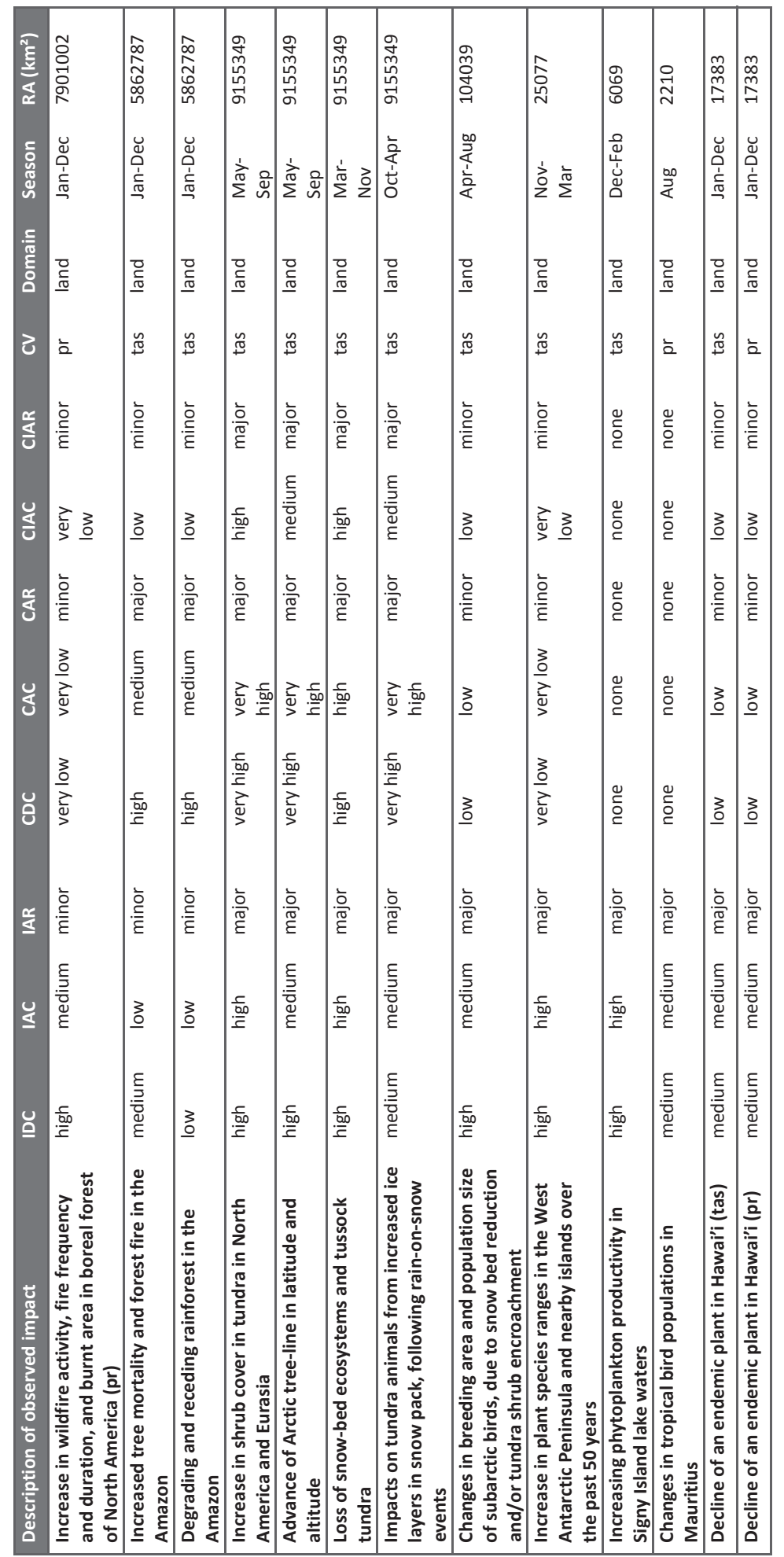




\section{Acknowledgments}

This thesis would not have been possible without the support and encouragement from a broad range of amazing people.

First and foremost, I want to thank my Promotor, Prof. Rik Leemans, for the possibility to anchor my research at the Wageningen University and Research Centre. His encouragement was vital in my decision to pursue a PhD based on the work I had done as a chapter scientist for the IPCC, and his continuous support in all scientific and editorial matters was invaluable. My Co-Promotor Prof. Max Auffhammer not only gave me the unique opportunity to visit UC Berkeley as an invited scholar, but also helped me steer clear of many cliffs and pitfalls, and always had the right response when doubt was taking over. Both my supervisors engaged with me way beyond expectations and contributed immensely to the successful completion of this thesis.

I want to thank the Co-Authors of the included publications, Max Auffhammer, Wolfgang Cramer, Christian Huggel, Andrew Solow and Dáithí Stone for their valuable contributions and excellent cooperation. I also extend my gratitude to the other members of the IPCC WGII Chapter 18 author team, Ulf Molau, Maria Assuncao Fuas da Silva Dias, Lourdes Tibig and Gary Yohe, and other experts from the WGII AR5 cycle, most of them active contributors to the cross-chapter working group on detection and attribution, namely Graham Cogley, Eberhard Faust, Jean Pierre Gattuso, Ove HoeghGuldberg, Yasuaki Hijoka, Lesley Hughes, David Karoly, Sari Kovats, Paul Leadley, David Lobell, Jose Marengo, John Morton, Leonard Nurse, Elvira Poloczanska, Hans Otto Pörtner, Andy Reisinger, Josef Settele, Arthur Webb and Mark Carey, who together laid the groundwork from which this thesis builds. However, not only did some of the world's most outstanding scientists share their expertise and engage in a continuous and lively debate on impact detection and attribution during the AR5 writing process, they also kept being responsive after the finalization and publication of AR5, enabling me to extend my work over such an incredibly broad area of topics and regions. I am humbled by such generosity.

I also want to thank the Co-Chairs of WGII, Chris Field and Vicente Barros, and the WGII technical support unit for setting up the chapter scientist program and the possibility to participate in the lead author meetings. The personal exchange was central to my research and facilitated the unusual quest of Chapter 18 to involve experts from all sectors and regions in their assessment. I also want to acknowledge Dave Dokken for his wisdom and humour, and Yuka Estrada for outstanding support with graphics.

I am grateful to the German Ministry of Education and Research for funding my position as a chapter scientist, and to the team of the German IPCC focal point, notably Dr Christiane Textor, for their 
excellent support and the unique chance to participate in the IPCC WGII approval plenary as a member of the German delegation.

The inspiring environment and the many brilliant colleagues at the Potsdam Institute for climate impact research supported my work in a multitude of ways. First of all, I want to thank my group leader, Dr Kirsten Thonicke, for her continuous interest and time, and for coaching me through the more difficult periods of this work. Our director Prof. Schellnhuber encouraged me to pursue this idea as a thesis, and the working group III TSU colleagues helped me reflect on the science-policy issues. The apt support of the IT crowd, Sandro Waldschmidt, Benjamin Kriemann and Dietmar Gibietz-Rheinbay, is what enables all of us to do our jobs properly. My colleagues Jacob Schewe, Isabel Weindl, Thomas Nocke, Christoph von Stechow, Jan Minx, Brigitte Knopf, Eva Schmid, Jascha Lehmann, Dim Coumou, Olivia Serdeczny, Patrick Eickemeier and Jessica Strefler contributed to my work by providing feedback on ideas, texts and graphics, and the opportunity to reflect on progress and the lack thereof.

To name all the friends, dance partners, sport buddies, therapists, housemates, family members and colleagues who have helped to sustain me as a reasonably sane human being over the last four years is not possible. So here I restrict the list of names to the slightly smaller group that was directly involved in processes and decisions regarding this thesis, and of course its finalization: Marc Gärtner, Christoph Arndt, Franzis Binder, Olaf Bernau, Indre Illig, Olivia Santen, Helen Sundermeyer, Veikko Heinz, Claudia Munz, Eckehard Pioch and Phillip Bedall. A special thanks to Lena Schulte-Uebbing for wonderful reviews, and for being my lifeline to Wageningen.

Finally, I want to highlight the contribution of my colleague Dáithí Stone to the analysis in Chapter 5, the method behind it, and virtually everything climate-related within this thesis. Thank you for bringing a long and winded journey to such a successful end.

And last but not least there is one person who continuously helped me keep up the spirit, inspired me to critically reflect every word and thought, guided me in making thorny decisions and above all shared his incredible sense of humour. I feel deeply honoured and grateful for the chance to work with him. He probably hates the word but I understand now what a mentor is.

Thank you, Andy. 


\section{About the author}

My academic career started at the Department of Physics at the University of Hamburg. However, when I realized that I was more interested in the interface between human society, technology and the natural environment than in abstract sciences, I enrolled at the Faculty of Process Sciences of the Technical University Berlin for a degree in Environmental Engineering. During my university years, volunteering and internships brought me to remote and less developed places, such as Indonesia, Nicaragua or Laos, raising my awareness of the social and environmental challenges related to poverty. I also studied and practiced low-impact living and communitarian ideas, and engaged locally and internationally in what was then the 'alter-globalisation movement'. After completing my TU Diplom (equivalent to a Master of Engineering) in 2004, I temporarily installed myself at a rural collective in North-East Germany, combining engineering work on solar PV installations and other green technologies with projects related to civil rights and cultural diversity. I also worked freelance in what is now called 'education for sustainable development'.

I then shifted my focus from 'act local' to 'think global'. My second degree, a Master of Science in "Global Change Management" at the University for Sustainable Development in Eberswalde, led me from the bottom-up world of grassroots movements to the top-down world of integrated assessment and Earth system modelling. I became involved in the UNFCCC process as an observer and was fascinated by the diplomatic realm of global governance. After graduation in 2009, I started my first science position at the Potsdam Institute for Climate Impact Research (PIK). Within the technical support unit of the Intergovernmental Panel on Climate Change (IPCC)'s working group III, I focused on the "Special Report on Renewable Energies and Climate Change Mitigation". In summer 2011, I had the chance to become a chapter scientist for the working group II Fifth Assessment Report. Still within PIK, I supported Prof. Cramer's Chapter 18 on "Detection and attribution of observed climate change impacts", and the coordination of German IPCC authors. My work for the IPCC was demanding yet it entailed wonderful opportunities and deep insights into international science-policy processes. The idea of a doctorate had been on my mind for some time, not least because of the joy and satisfaction that I found in my concurrent teaching appointments at Eberswalde University. So when the great team of Chapter 18 collectively encouraged me to pursue this idea, I set to work immediately. Now after four exciting years of research into the complexity of impact detection and attribution, I am looking forward to further engage with the challenges of making the world a better place for all. 


\section{Selected publications}

2015 Hansen, G., D. Stone, M. Auffhammer, C. Huggel, W. Cramer, 2015: Linking local impacts to climate change - a guide to attribution. Regional Environmental Change, online. doi:10.1007/s10113-0150760-y.

Hansen, G., Cramer, W., 2015: The global distribution of observed climate change impacts. Commentary, Nature Climate Change, 5:182-185.

Hansen, G., 2015: The evolution of the evidence base for observed climate change impacts. Current Opinion in Environmental Sustainability, 14:187-197, online. doi:10.1016/j.cosust.2015.05.005

Hansen, G. and Stone, DA.: Assessing the observed impact of anthropogenic climate change (submitted to Nature Climate Change), in review.

Stone, DA and Hansen, G: Rapid systematic assessment of the detection and attribution of regional anthropogenic climate change (submitted to Climate Dynamics), in review.

Huggel, C., D. Stone, H. Eicken, G. Hansen, 2015: Potential and limitations of the attribution of climate change impacts for informing loss and damage discussions and policies. Climatic Change, Special Issue on Climate Justice, online. doi: 10.1007/s10584-015-1441-z.

Hirschfeld, J., D. Messner, G. Hansen: Die klimaresiliente Gesellschaft - Transformation und Systemänderungen. In Brasser, G., Jacob, D. (eds) Klimabericht: Assessment für Deutschland, Springer, Germany (in press).

Schulte-Uebbing, L., G. Hansen, A. Hernández, M. Winter, M.: Chapter Scientists in the IPCC AR5 Experience and Lessons Learned. Current Opinion on Environmental Sustainability, online. doi:10.1016/j.cosust.2015.06.012.

2014 Hansen, G., Auffhammer, M., Solow, A., 2014: On the attribution of an extreme event to climate change. Journal of Climate, 27:8297-8301.

Contributing Author to Cramer, W et al., 2014: Detection and attribution of observed impacts. In: Climate Change 2014: Impacts, Adaptation and Vulnerability. Contribution of Working Group II to the Fifth Assessment Report of the Intergovernmental Panel on Climate Change [C.B. Field, V. Barros, D.J. Dokken, et al (eds)]. Cambridge University Press, Cambridge, UK, and New York, NY, USA.

2013 Huggel, C., Stone, D., Auffhammer, M., Hansen, G., 2013: Loss and damage attribution. Nature Climate Change, 3(8):694-696.

Hansen, G., D. Stone, M. Auffhammer, 2013: Detection and attribution of climate change impacts is a universal discipline possible? Impacts World Conference, Potsdam.

Stone, D., M. Auffhammer, M. Carey, G. Hansen, C. Huggel, W. Cramer, D. Lobell, U. Molau, A. Solow, L. Tibig, and G. Yohe, 2013: The challenge to detect and attribute effects of climate change on human and natural systems. Climatic Change, 121(2).

2012 Hansen, G., M. Jakob, S. Kadner, 2012: Sustainable Development as a Cornerstone of a Future Energy System. In Climate Change, Justice and Sustainability [Edenhofer, O.; Wallacher, J.; LotzeCampen, H.; Reder, M.; Knopf, B.; Müller, J. (Eds.)], Springer, Germany.

O. Edenhofer, R. Pichs-Madruga, Y. Sokona, C. Field, V. Barros, T.F. Stocker, Q. Dahe, J. Minx, K. Mach, G.-K. Plattner, S. Schlömer, G. Hansen, M. Mastrandrea (eds.). Meeting Report of the IPCC Expert Meeting on Geoengineering. IPCC Working Group III Technical Support Unit, Potsdam Institute for Climate Impact Research, Potsdam, Germany, pp. 99.

2011 IPCC Special Report on Renewable Energy Sources and Climate Change Mitigation (SRREN) [O. Edenhofer, R. Pichs-Madruga, Y. Sokona, K. Seyboth, P. Matschoss, S. Kadner, T. Zwickel, P. Eickemeier, G. Hansen, S. Schlomer, C. von Stechow (eds)], Cambridge University Press, Cambridge, United Kingdom and New York, NY, USA.

- Author of the Summary for Policymakers, and the Technical Summary of the IPCC SRREN,

- Contributing Author of IPCC SRREN Chapter 9, Renewable Energy in the Context of Sustainable Development. 


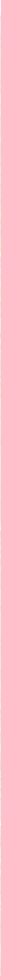

